Subalgebras Maximais das

\title{
Álgebras de Lie Semisimples, Quebra de Simetria e o Código Genético
}

Fernando Martins Antoneli Júnior

$$
\begin{gathered}
\text { DISSERTAÇÃO APRESENTADA } \\
\text { AO } \\
\text { INSTITUTO DE MATEMÁTICA E ESTATÍSTICA }
\end{gathered}
$$

DA

UNIVERSIDADE DE SÃO PAULO

PARA

OBTENÇÃO DO GRAU DE MESTRE

EM

MATEMÁTICA APLICADA

\section{Área de Concentração: Matemática Aplicada Orientador: Prof. Dr. Michael Forger}

Durante a elaboração deste trabalho o autor recebeu apoio financeiro da CAPES

-São Paulo, agosto de 1998- 



\title{
Subalgebras Maximais das Álgebras de Lie Semisimples, Quebra de Simetria e o Código Genético
}

\author{
Este exemplar corresponde à redação final da \\ dissertação devidamente corrigida e defendida \\ por Fernando Martins Antoneli Júnior \\ e aprovada pela comissão julgadora.
}

São Paulo, 30 de agosto de 1998.

\section{COMISSÃO JULGADORA}

- Prof. Dr. Frank Michael Forger (orientador) - IME-USP

- Prof. Dr. José Eduardo Martinho Hornos - IFSC-USP

- Prof. Dr. Said Najati Sidki - UnB 



\title{
Resumo
}

O propósito deste trabalho é dar uma contribuição ao projeto iniciado por Hornos \& Hornos que visa explicar as degenerescências do código genético como resultado de sucessivas quebras de simetria ocorridas durante sua evolução. O modelo matemático usado requer a construção de todas as representações irredutíveis de dimensão 64 das álgebras de Lie simples (chamadas representações de códons) e a análise de suas regras de ramificação sob redução a subalgebras. A classificação de todas as possibilidades é baseada na classificação das subalgebras maximais das álgebras de Lie semisimples obtida por Dynkin. No presente trabalho, os resultados de Dynkin são apresentados em linguagem e notação moderna e são aplicados ao problema de construir todas as possíveis cadeias de subalgebras maximais das álgebras de Lie simples $B_{6}=\mathfrak{s o}(13)$ e $D_{7}=\mathfrak{s o}(14)$ e de identificar aquelas que reproduzem as degenerescências do código genético.

\begin{abstract}
The purpose of this work is to make a contribution to the project initiated by Hornos \& Hornos which aims at explaining the degeneracy of the genetic code as the result of a sequence of symmetry breakings that occurred during its evolution. The mathematical model employed requires the construction of all 64-dimensional irreducible representations of simple Lie algebras (called codon representations) and the analysis of their branching rules under reduction to subalgebras. The classification of all possibilities is based on Dynkin's classification of the maximal subalgebras of semisimple Lie algebras. In the present work, Dynkin's results are presented in modern language and notation and are applied to the problem of constructing all possible chains of maximal subalgebras of the simple Lie algebras $B_{6}=\mathfrak{s o}(13)$ and $D_{7}=\mathfrak{s o}(14)$ and of identifying all those that reproduce the degeneracies of the genetic code.
\end{abstract}



Aos meus pais, Fernando e Eideni. 

.. não é nada natural que existam "leis da natureza", muito menos ainda que o homem seja capaz de descobri-las.

E. P. Wigner. 



\section{Agradecimentos}

Ao Prof. Michael Forger, meu orientador, pelo incentivo, confiança, compreensão, paciência. A sua experência como pesquisador e professor tornou possível a realização desta dissertação e contribuiu para a minha formação matemática e cultural.

Aos meus pais, Fernando e Eideni, a quem também dedico este trabalho, pelo apoio e compreensão desde que eu decidi seguir a carreia acadêmica.

Ao Daniel, Samuel, Sidnei, Walquíria, Cecília, Irene, Zé, Jorge, e Regina, colegas de pós-graduação, pela ajuda nas disciplinas e pelas horas de estudo em grupo, mas também pelas festas e momentos de descontração durante estes dois anos de estudo. Ao Raul, Rodrigo, e Lílian, colegas do tempo da graduação que também ingressaram na pós-graduação, pelo apoio e companheirismo. Ao Klaus, Fátima e Karin, colegas de CPUSP, onde descarregamos na bola de volei as tensões e irritações do dia a dia. Aos colegas Sandro e Mário, pela ajuda e pelas discussões sobre matemática, física, filosofia, biologia, etc, que foram muito úteis na hora de escrever esta dissertação.

Aos professores da graduação, em especial à Prof. Zara, a Prof. Maria Lucia, o Prof Francisco Miraglia, o Porf. Jacob Zimbarg, o Prof. Ivan de Camargo, que inspiram seus alunos sendo profissionais exemplares. 



\section{Conteúdo}

Introdução

1 Subalgebras Maximais das

Álgebras de Lie Semisimples $\quad 1$

1.1 Conceitos Básicos . . . . . . . . . . . . . . . . . 1

1.2 Estrutura das Subalgebras Maximais das Álgebras de Lie Semisimples . . . . . . . . . . . . . . . . . . . 7

1.3 Álgebras de Lie Redutivas e Compactas . . . . . . . . . . . . . . . 15

1.4 Classificação das Subalgebras Maximais das Álgebras de Lie Simples . . . . . . . . . . . . . . . . . . . . . . . 23

2 Quebra de Simetrias no Código Genético 41

2.1 Modelo Geral de Quebra de Simetrias . . . . . . . . . . . . . . . . . 41

2.2 Produto Tensorial e Restrição de Representações . . . . . . . . . . . . . . . . 44

2.3 Subalgebras Maximais das Álgebras Simples para o Código Genético . . . . . . . . . . . . . . . . . 47

2.4 Cadeias de Subalgebras Semisimples para o Código Genético . . . . . . . . . . . . . . . . . . . . . 62

A Cadeias Sobreviventes de $B_{6} \quad 83$

B Cadeias Sobreviventes de $D_{7} \quad 93$ 
Bibliografia 


\section{Lista de Figuras}

1.1 Peso $(1,1)$ da álgebra excepcional $G_{2} \ldots \ldots \ldots \ldots \ldots$ 



\section{Lista de Tabelas}

1.1 Numeração das raizes simples no diagrama de Dynkin. . . . . . . . . . . 5

1.2 Álgebras de Lie clássicas. . . . . . . . . . . . . . . . . . . . 25

1.3 Representações de dimensão mínima das álgebras clássicas. . . . . . . . . . . 26

1.4 Subalgebras maximais redutíveis. . . . . . . . . . . . . . . . 28

1.5 Subalgebras maximais irredutíveis não simples. . . . . . . . . . . . . . . . . 31

1.6 Subalgebras irredutíveis simples não maximais. . . . . . . . . . . . . . . . . 35

1.7 Subalgebras maximais das álgebras de Lie excepcionais. . . . . . . . . . . . . 37

1.8 Espaços simétricos riemannianos excepcionais. . . . . . . . . . . . . 38

2.1 Representações de Códons. . . . . . . . . . . . . . . . . . . . . . . . . . 48

2.2 Subalgebras maximais de $A_{2}=\mathfrak{s u}(3) \ldots \ldots \ldots$. . . . . . . . 51

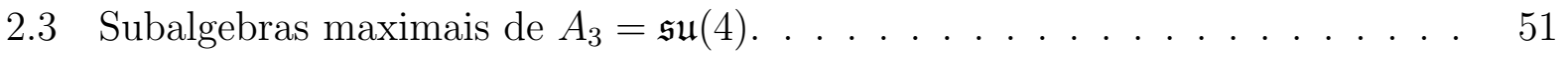

2.4 Subalgebras maximais de $A_{4}=\mathfrak{s u}(5) \ldots \ldots \ldots$. . . . . . . . . 51

2.5 Subalgebras maximais de $A_{5}=\mathfrak{s u}(6)$. . . . . . . . . . . . . . . 52

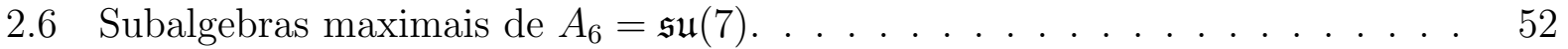

2.7 Subalgebras maximais de $B_{2}=\mathfrak{s o}(5) \ldots \ldots \ldots$. . . . . . . . . 53

2.8 Subalgebras maximais de $B_{3}=\mathfrak{s o}(7) \ldots \ldots \ldots$. . . . . . . . . . 53

2.9 Subalgebras maximais de $B_{4}=\mathfrak{s o}(9) \ldots \ldots \ldots$. . . . . . . . . . . . . 53

2.10 Subalgebras maximais de $B_{5}=\mathfrak{s o}(11)$. . . . . . . . . . . . . . . . . 54

2.11 Subalgebras maximais de $B_{6}=\mathfrak{s o}(13) \ldots \ldots \ldots$. . . . . . . . . . . . 54 
2.12 Subalgebras maximais de $C_{3}=\mathfrak{s p}(6) \ldots \ldots \ldots$. . . . . . . . . 55

2.13 Subalgebras maximais de $D_{4}=\mathfrak{s o}(8) \ldots \ldots$. . . . . . . . . . . 56

2.14 Subalgebras maximais de $D_{5}=\mathfrak{s o}(10)$. . . . . . . . . . . . . . . . . . . 56

2.15 Subalgebras maximais de $D_{6}=\mathfrak{s o}(12)$. . . . . . . . . . . . . . . . . . . . . 57

2.16 Subalgebras maximais de $D_{7}=\mathfrak{s o}(14) \ldots \ldots \ldots$. . . . . . . . . 57

2.17 Subalgebras maximais de $G_{2}$. . . . . . . . . . . . . . . . . . . 58

2.18 Subalgebras maximais de $C_{32}=\mathfrak{s p}(64) \ldots \ldots$. . . . . . . . . . . . 59

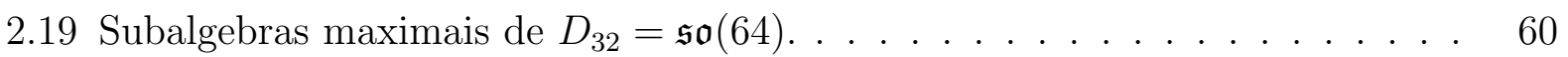

2.20 Subalgebras maximais de $A_{63}=\mathfrak{s u}(64) \ldots \ldots$. . . . . . . . . . . 61

2.21 Multipletos que aparecem em $B_{6}$ e $D_{7} \ldots \ldots$. . . . . . . . . . . . 68

2.22 Cadeia 1 de $D_{7} \ldots \ldots \ldots$. . . . . . . . . . . . . . . . . . . . . . . . . . 69

2.23 Cadeia 2 de $D_{7} \ldots \ldots \ldots$. . . . . . . . . . . . . . . . . 71

2.24 Cadeia 3 de $D_{7} \ldots \ldots \ldots \ldots$. . . . . . . . . . . . . . . . 74

2.25 Cadeia 4 de $D_{7} \ldots \ldots \ldots \ldots \ldots$. . . . . . . . . . . . . 76

2.26 Cadeia 5 de $D_{7} \ldots \ldots \ldots \ldots$. . . . . . . . . . . . . . . 79 


\section{Introdução}

O propósito deste trabalho é dar uma contribuição ao projeto iniciado por Hornos \& Hornos [22], onde é proposta uma nova abordagem ao problema da evolução do código genético. Neste trabalho, que teve grande repercussão na comunidade científica internacional [33], [50], os autores apresentam um modelo algébrico que interpreta as degenerescências do código genético como sendo o resultado de um processo evolutivo acompanhado por uma sequência de quebras de simetria e, portanto, intimamente relacionado com a teoria dos grupos. O principal resultado deste artigo é que no contexo dos grupos de Lie compactos existe um grupo, juntamente com uma cadeia descendente de subgrupos fechados conexos, que chega a reproduzir as degenerescências do código genético: este modelo é baseado no grupo simplético $S p(6)$ e inclui a hipótese de um "congelamento" do processo de quebra no último passo. Mais recentemente, foi proposta uma ligeira generalização do esquema, dentro da qual surge uma nova cadeia, desta vez baseada no grupo $G_{2}$ [14].

Dentro deste contexto, nosso objetivo é abordar a questão da sistematização da análise que foi feita em [22]. Há dois aspectos que serão discutidos em capítulos separados e que a primeira vista são independentes, mas na verdade estão interligados pela aplicação dos resultados ao modelo algébrico para a evolução do código genético. No primeiro capítulo, apresentamos os resultados de Dynkin sobre a classificação das subalgebras semisimples maximais das álgebras de Lie semisimples [10,11]. Estes resultados participam indiretamente da análise feita em [22], pois esta se baseia nas tabelas de McKay \& Patera [35] que, por sua vez, foram construídas utilizando a classificação de Dynkin. A inclusão deste assunto se deve à tentativa de compreender melhor a estrutura das subalgebras maximais das álgebras de Lie semisimples, o que se mostrou um desafio devido à dificuldade de leitura dos trabalhos

originais de Dynkin. A nossa meta aqui é apresentar os resultados de Dynkin numa linguagem moderna e de modo a facilitar a aplicação em problemas concretos. Não apresentamos as demonstrações de todos os teoremas, pois isto nos levaria muito longe, fugindo assim do objetivo principal do presente trabalho. 
Um destes teoremas, porém, tem um papel fundamental na discussão do segundo capítulo e por isto damos também a sua demonstração: ele descreve a estrutura geral das subalgebras maximais das álgebras de Lie semisimples e reduz o problema da sua classificação ao da classificação das subalgebras maximais das álgebras de Lie simples. A parte do trabalho de Dynkin que apresentaremos sem demonstração é justamente a classificação das subalgebras maximais das álgebras de Lie simples. Por fim, mostraremos que a classificação das subalgebras semisimples maximais das álgebras de Lie semisimples complexas é essencialmente equivalente à classificação dos subgrupos conexos maximais (fechados) dos grupos de Lie compactos. Isto justifica a afirmação de que a análise das degenerescências do código genético no contexto das álgebras de Lie semisimples é, em princípio, equivalente à análise no contexto dos grupos de Lie compactos.

No segundo capítulo daremos uma sistematização da análise das cadeias descendentes de subalgebras maximais das álgebras de Lie simples que admitem representações irredutíveis de dimensão 64: são estes os candidatos para um modelo algébrico para a evolução do código genético. Esta análise tem por objetivo identificar aquelas cadeias que reproduzem a degenerescência do código genético. Como o número de cadeias cresce muito em função do posto da álgebra, torna-se necessário formular critérios [23] para eliminar cadeias que, por algum motivo ou outro, são incapazes de reproduzir a distribuição de multipletos encontrada no código genético, independentemente dos eventuais passos posteriores no processo de quebra de simetrias. Em [23], tais critérios de eliminação prévia - ou seja, de identificação de cadeias "não-sobreviventes" - foram explicitamente formulados e aplicados às cadeias oriundas das álgebras de Lie simples de posto baixo (menor ou igual a 3) que possuem representações irredutíveis de dimensão 64. No caso das álgebras de Lie simples de posto médio que possuem representações irredutíveis de dimensão 64 , que são $B_{6}=\mathfrak{s o}(13)$ e $D_{7}=\mathfrak{s o}(14)$, essa simplificação torna-se ainda mais importante, pois mesmo após a aplicação dos critérios de eliminação estipulados em [23], encontramos várias cadeias sobreviventes, 2 para $B_{6}$ e 9 para $D_{7}$, que precisam ser analisadas até o fim, sendo que uma das cadeias para $D_{7}$ realmente fornece as degenerescências do código genético [23]. Os detalhes desta análise podem ser encontrados na dissertação de Mestrado de L. Braggion [3].

Uma das metas principais de presente trabalho é incluir nesta análise uma outra possibilidade de construir subalgebras maximais, possibilidade esta que não foi contemplada em [22] (em [23] ela já foi incorporada ao esquema geral) mas que é legítima de acordo com o teorema de estrutura das subalgebras maximais das álgebras de Lie semisimples. Com esta extensão, surgem novas cadeias, aumentando mais ainda o número de cadeias a serem analisadas e, no caso de $D_{7}$, aparecem mais 7 cadeias que realmente fornecem as degenerescências do código genético. Por outro lado, é possível garantir que com essa extensão, a análise está completa: não há nenhuma outra possibilidade de construir cadeias descendentes de subalgebras das álgebras de Lie simples que possuem representações irredutíveis de dimensão 64 capazes de fornecer as degenerescências do código genético. 
Desta forma, a implementação do projeto proposto em [22] no contexto dos grupos de Lie compactos está completa. Porém já foi observado [22] que uma análise da mesma natureza pode ser realizada em outros contextos, tais como: grupos finitos, grupos quânticos e superalgebras de Lie. Algumas destas opções estão atualmente sob investigação.

Quanto ao estilo de apresentação escolhido, optamos por não incluir conceitos e resultados básicos da teoria geral dos grupos e álgebras de Lie, tendo em vista a extensa literatura sobre esta área [2], [6], [7], [15], [19], [20], [24], [39], [45], [44], [47], [48], [54], em particular os clássicos [8], [58], [61]. A única exceção se dá com respeito aos resultados de Dynkin sobre a classificação das subalgebras maximais das álgebras de Lie semisimples, cujas fontes principais ainda são os trabalhos originais de Dynkin $[10,11]$ complementados por alguns "surveys" [53], [42]. Estes dois últimos trabalhos fornecem uma visão geral dos teoremas de Dynkin (sem os detalhes das demonstrações) e servem de guia para a leitura de [10, 11], sendo que [42] apresenta estes resultados em linguagem moderna. Mas mesmo neste caso, a apresentação procura dar ênfase à aplicação dos teoremas em casos concretos, colocando os resultados em tabelas de fácil consulta. Também incluímos uma generalização da teoria de formas reais compactas para álgebras de Lie redutivas, o que pemite extender a teoria de Dynkin, originalmente formulada no contexto das álgebras de Lie semisimples, à classe das álgebras de Lie redutivas e leva a uma formulação mais uniforme e mais transparente dos resultados. Finalmente, apresentamos no segundo capítulo uma lista de todas as subalgebras maximais das álgebras de Lie simples que aparecem dentro de alguma cadeia oriunda de uma álgebra de Lie simples que possui uma representação irredutível de dimensão 64, com a finalidade de exemplificar a aplicação dos teoremas de classificação que enunciamos no primeiro capítulo. Estas listas de subalgebras fazem parte das tabelas de McKay \& Patera [35], onde porém não se explica como foram construídas. 

CAPÍtulo 1

\section{Subalgebras Maximais das Álgebras de Lie Semisimples}

Neste capítulo, as álgebras de Lie podem ser reais ou complexas, a menos que se mencione explicitamente o corpo base, e todas as representações são de dimensão finita.

\subsection{Conceitos Básicos}

Nesta seção daremos algumas definições elementares que constam da literatura especializada em álgebras de Lie, com o intuito de uniformizar nossa terminologia.

Se $\mathfrak{h}$ é uma subalgebra de uma álgebra de Lie $\mathfrak{g}$ e $\varphi$ é um automorfismo de $\mathfrak{g}$ então $\varphi(\mathfrak{h})$ também é uma subalgebra de $\mathfrak{g}$. Portanto, como existe uma infinidade de automorfismos em uma álgebra de Lie, existe uma infinidade de subalgebras isomorfas entre si. É fácil ver que tais subalgebras formam uma classe de equivalência, e por isso basta classificar estas classes de equivalência para que se tenha um classificação das subalgebras.

Definição 1.1.1. Seja $\mathfrak{g}$ uma álgebra de Lie. Sejam $\mathfrak{g}_{1}$ e $\mathfrak{g}_{2}$ duas subalgebras de $\mathfrak{g}$. Dizemos que $\mathfrak{g}_{1}$ e $\mathfrak{g}_{2}$ são conjugadas em $\mathfrak{g}$ se existe um automorfismo interno $\sigma$ de $\mathfrak{g}$ tal que $\mathfrak{g}_{1}=\sigma\left(\mathfrak{g}_{2}\right)$.

É fácil mostrar que a conjugação constitui uma relação de equivalência no conjunto das subalgebras de uma álgebra de Lie, e as classes de equivalência correspondentes são chamadas classes de conjugação de subalgebras.

As vezes é comum que uma subalgebra seja definida através de um homomorfismo, portanto precisamos formular uma noção análoga de conjugação quando estamos nesta situação. 
Definição 1.1.2. Sejam $\mathfrak{f}$ e $\mathfrak{g}$ duas álgebras de Lie. Uma inclusão de $\mathfrak{f}$ em $\mathfrak{g}$ é um homomorfismo injetor $\varphi: \mathfrak{f} \rightarrow \mathfrak{g}$ de álgebras de Lie.

É claro que, se $\varphi$ é uma inclusão de $\mathfrak{f}$ em $\mathfrak{g}$, o conjunto $\varphi(\mathfrak{f})$ é uma subalgebra de $\mathfrak{g}$ isomorfa a $\mathfrak{f}$. Por outro lado se $\mathfrak{f} \subset \mathfrak{g}$, então a aplicação identidade de $\mathfrak{g}$ restrita a $\mathfrak{f}$ é uma inclusão de $\mathfrak{f}$ em $\mathfrak{g}$.

Definição 1.1.3. Sejam $\mathfrak{f}$ e $\mathfrak{g}$ duas álgebras de Lie $e \varphi, \psi: \mathfrak{f} \rightarrow \mathfrak{g}$ duas inclusões de $\mathfrak{f}$ em $\mathfrak{g}$. Dizemos que $\varphi$ e $\psi$ são conjugados se existe um automorfismo interno $\sigma$ de $\mathfrak{g}$ tal que $\varphi=\sigma \circ \psi$.

A proposição seguinte é a chave para entender como se relacionam os conceitos de conjugação de subalgebras e conjugação de inclusões.

Proposição 1.1.1. Sejam $\mathfrak{g}$ e $\mathfrak{f}$ duas álgebras de Lie. Sejam $\varphi, \psi: \mathfrak{f} \rightarrow \mathfrak{g}$ duas inclusões e $\sigma \in \operatorname{Aut}(\mathfrak{g})$. Então $\varphi(\mathfrak{f})=\sigma(\psi(\mathfrak{f}))$ se e somente se $\varphi=\sigma \circ \psi \circ \tau$, onde $\tau \in \operatorname{Aut}(\mathfrak{f})$.

DemonstraÇÃo. É óbvio que $\varphi=\sigma \circ \psi \circ \tau$ implica $\varphi(\mathfrak{f})=\sigma(\psi(\mathfrak{f}))$. Reciprocamente, suponhamos que $\varphi(\mathfrak{f})=\sigma(\psi(\mathfrak{f}))$, como $\varphi$ e $\sigma \circ \psi$ são inclusões temos que:

$$
\begin{gathered}
\tilde{\varphi}: \mathfrak{f} \longrightarrow \varphi(\mathfrak{f}) \\
\sigma \circ \tilde{\psi}: \mathfrak{f} \longrightarrow \varphi(\mathfrak{f})
\end{gathered}
$$

são isomorfismos de álgebras de Lie. Consideremos $(\sigma \circ \tilde{\psi})^{-1}: \varphi(\mathfrak{f}) \rightarrow \mathfrak{f}$ o isomorfismo inverso a $\sigma \circ \tilde{\psi}$; então o isomorfismo de álgebras de Lie dado por

$$
\tau=(\sigma \circ \tilde{\psi})^{-1} \circ \tilde{\varphi}: \mathfrak{f} \longrightarrow \mathfrak{f}
$$

é um automorfismo de $\mathfrak{f}$ que satisfaz $\varphi=\sigma \circ \psi \circ \tau$.

Corolário 1.1.1. Sejam $\mathfrak{g}$ e $\mathfrak{f}$ duas álgebras de Lie e $\varphi, \psi: \mathfrak{f} \rightarrow \mathfrak{g}$ duas inclusões. Se todos os automorfismos de $\mathfrak{f}$ são internos, as subalgebras $\varphi(\mathfrak{f})$ e $\psi(\mathfrak{f})$ são conjugadas em $\mathfrak{g}$ se $e$ somente se as inclusões $\varphi$ e $\psi$ são conjugadas.

DemonstraÇÃo. Se as inclusões $\varphi$ e $\psi$ são conjugadas é óbvio que as subalgebras $\varphi(\mathfrak{f})$ e $\psi(\mathfrak{f})$ são conjugadas. Reciprocamente, se $\varphi(\mathfrak{f})$ e $\psi(\mathfrak{f})$ são subalgebras conjugadas, a proposição anterior implica que existem $\tau \in \operatorname{Aut}(\mathfrak{f})$ e $\sigma \in \operatorname{Aut}(\mathfrak{g})$ tais que $\varphi=\sigma \circ \psi \circ \tau$. Mas por hipótese $\tau$ é um automorfismo interno de $\mathfrak{f}$, ou seja $\tau=\exp \operatorname{ad}\left(Y_{1}\right) \cdot \ldots \cdot \exp \operatorname{ad}\left(Y_{k}\right) \operatorname{com} Y_{1}, \ldots, Y_{k} \in \mathfrak{f}$. Podemos considerar $\tilde{\tau}=\exp \operatorname{ad}\left(\psi\left(Y_{1}\right)\right) \cdot \ldots \cdot \exp \operatorname{ad}\left(\psi\left(Y_{k}\right)\right)$, que é um automorfismo interno de $\mathfrak{g}$. Porém $\tilde{\tau}$ restrito à subalgebra $\psi(\mathfrak{f})$ é um automorfismo interno desta subalgebra. 
Definimos então $\varsigma=\sigma \circ \tilde{\tau}$, que também é um automorfismo interno de $\mathfrak{g}$. Seja agora $X \in \mathfrak{f}$, então

$$
\begin{aligned}
(\varsigma \circ \psi)(X) & =(\sigma \circ \tilde{\tau} \circ \psi)(X) \\
& =\sigma\left(\left(\exp \operatorname{ad}\left(\psi\left(Y_{1}\right)\right) \cdot \ldots \cdot \exp \operatorname{ad}\left(\psi\left(Y_{k}\right)\right)\right)(\psi(X))\right) \\
& =\sigma\left(\psi\left(\exp \operatorname{ad}\left(Y_{1}\right) \cdot \ldots \cdot \exp \operatorname{ad}\left(Y_{k}\right)\right)(X)\right) \\
& =\sigma((\psi \circ \tau)(X))=(\sigma \circ \psi \circ \tau)(X)=\varphi(X),
\end{aligned}
$$

ou seja, temos a conjugação de inclusões $\varphi=\varsigma \circ \psi$.

Quando $\mathfrak{f}$ possuir automorfismos externos devemos determinar as classes de conjugação de homomorfismos e depois verificar quais classes são transformadas umas nas outras por automorfismos externos de $\mathfrak{f}$. Isto esclarece, em princípio, a relação entre conjugação de subalgebras e conjugação de inclusões.

Há ainda uma noção mais fraca que a de conjugação que foi introduzida por Dynkin e é muito importante para a formulação do teorema de classificação das subalgebras maximais das álgebras de Lie excepcionais.

Definição 1.1.4. Sejam $\mathfrak{g}$ uma álgebra de Lie e $\mathfrak{f}_{1}, \mathfrak{f}_{2} \subset \mathfrak{g}$ duas subalgebras. Dizemos que $\mathfrak{f}_{1}$ $e \mathfrak{f}_{2}$ são linearmente conjugadas ou L-conjugadas se para toda representação $\rho: \mathfrak{g} \rightarrow \mathfrak{g l}(V)$ em um espaço vetorial complexo $V$ as subalgebras $\rho\left(\mathfrak{f}_{1}\right)$ e $\rho\left(\mathfrak{f}_{2}\right)$ são conjugadas em $\mathfrak{g l}(V)$. Analogamente, se $\mathfrak{f}$ é uma álgebra de Lie e $\varphi_{1}, \varphi_{2}: \mathfrak{f} \rightarrow \mathfrak{g}$ são inclusões dizemos que $\varphi_{1}$ e $\varphi_{2}$ são linearmente conjugadas ou L-conjugadas se para toda representação $\rho: \mathfrak{g} \rightarrow \mathfrak{g l}(V)$ em um espaço vetorial complexo $V$ as representações $\rho \circ \varphi_{1}: \mathfrak{f} \rightarrow \mathfrak{g l}(V)$ e $\rho \circ \varphi_{2}: \mathfrak{f} \rightarrow \mathfrak{g l}(V)$ são equivalentes.

A L-conjugação também é uma relação de equivalência no conjunto das subalgebras de uma álgebra de Lie. É fácil mostrar que se duas subalgebras são conjugadas então elas são $L$-conjugadas, porém a recíproca é falsa. O máximo que se pode dizer, em geral, é que toda classe de $L$-conjugação é a reunião de certas classes de conjugação.

Consideremos uma álgebra de Lie $\mathfrak{g}$ e fixemos uma subalgebra $\mathfrak{f} \subset \mathfrak{g}$. Seja $\rho: \mathfrak{g} \rightarrow \mathfrak{g l}(V)$ uma representação de $\mathfrak{g}$ em um espaço vetorial complexo $V$. Vamos definir a restrição da representação $\rho$ à subalgebra $\mathfrak{f}$ pondo

$$
\operatorname{Res}_{\mathfrak{f}}^{\mathfrak{g}}(\rho): \mathfrak{f} \rightarrow \mathfrak{g l}(V) .
$$

Claramente $\operatorname{Res}_{\mathfrak{f}}^{\mathfrak{g}}(\rho)$ é uma representação de $\mathfrak{f}$ em $V$. Supondo agora que $\rho$ é irredutível e que $\operatorname{Res}_{\mathfrak{f}}^{\mathfrak{g}}(\rho)$ é completamente redutível definimos a regra de ramificação de $\rho$ com respeito $a \mathfrak{f}$ como sendo o conjunto dos pares ordenados de representações irredutíveis e respectivas multiplicidades $\left\{\left(\pi_{\ell}, c_{\ell}\right) \mid \ell=1, \ldots, n\right\}$ de $\mathfrak{f}$ tais que

$$
\operatorname{Res}_{\mathfrak{f}}^{\mathfrak{g}}(\rho)=\bigoplus_{\ell=1}^{n} c_{\ell} \pi_{\ell}
$$


É claro que a regra de ramificação só depende da representação $\rho$ e da classe de $L$-conjugação de $\mathfrak{f}$.

Introduziremos agora uma notação que será muito útil para representar regras de ramificação. Suponhamos que $\mathfrak{g}$ seja uma álgebra de Lie simples de posto $r$ e fixemos uma subalgebra de Cartan $\mathfrak{h}$. Seja $\Delta \subset \mathfrak{h}^{*}$ o sistema de raizes de $\mathfrak{g}$ e $\Pi=\left\{\alpha_{1}, \ldots, \alpha_{r}\right\}$ um conjunto de raizes simples em $\Delta$. Seja $(\cdot, \cdot)$ um produto escalar em $\mathfrak{h}^{*}$, invariante sob a ação do grupo de Weyl $\mathcal{W}$ de $\Delta$ e normalizado pela condição

$$
(\alpha, \alpha)=2
$$

para toda raíz longa $\alpha \in \Delta$ : existe um único produto escalar em $\mathfrak{h}^{*}$ com esta normalização, chamado produto escalar padrão. Considere a base $\Pi^{\prime}=\left\{\lambda_{1}, \ldots, \lambda_{r}\right\}$ de $\mathfrak{h}^{*}$ definida por

$$
\left(\alpha_{i}, \lambda_{j}\right)=\delta_{i j}
$$

os elementos desta base chamam-se pesos fundamentais. Para $\vartheta \in \mathfrak{h}^{*}$ qualquer, se escrevermos

$$
\vartheta=\sum_{i=1}^{r} p_{i} \lambda_{i}
$$

temos que

$$
p_{i}=\left(\vartheta, \alpha_{i}\right) .
$$

Como as raizes simples são os vértices do diagrama de Dynkin de $\mathfrak{g}$ e os pesos fundamentais correpondem aos mesmos vértices do diagrama de Dynkin através da fórmula (1.1), podemos então representar um peso graficamente, desenhando o diagrama de Dynkin e rotulando o vértice $i$ com o coeficiente $p_{i}$. Para isto precisamos fixar uma numeração das raizes simples no diagrama de Dynkin: a tabela 1.1 apresenta uma tal numeração. Assim podemos, por exemplo, escrever o peso $\lambda_{1}+\lambda_{2}$ da álgebra $G_{2}$ da seguinte forma:

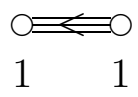

Figura 1.1: Peso $(1,1)$ da álgebra excepcional $G_{2}$.

Agora, pelo teorema do peso máximo de Cartan, um elemento $\vartheta \in \mathfrak{h}^{*}$ é o peso máximo de uma representação irredutível ${ }^{1}$ de $\mathfrak{g}$ se e somente se os números $p_{i}$ são inteiros

\footnotetext{
${ }^{1}$ Lembremos que todas as representações consideradas neste trabalho são de dimensão finita.
} 


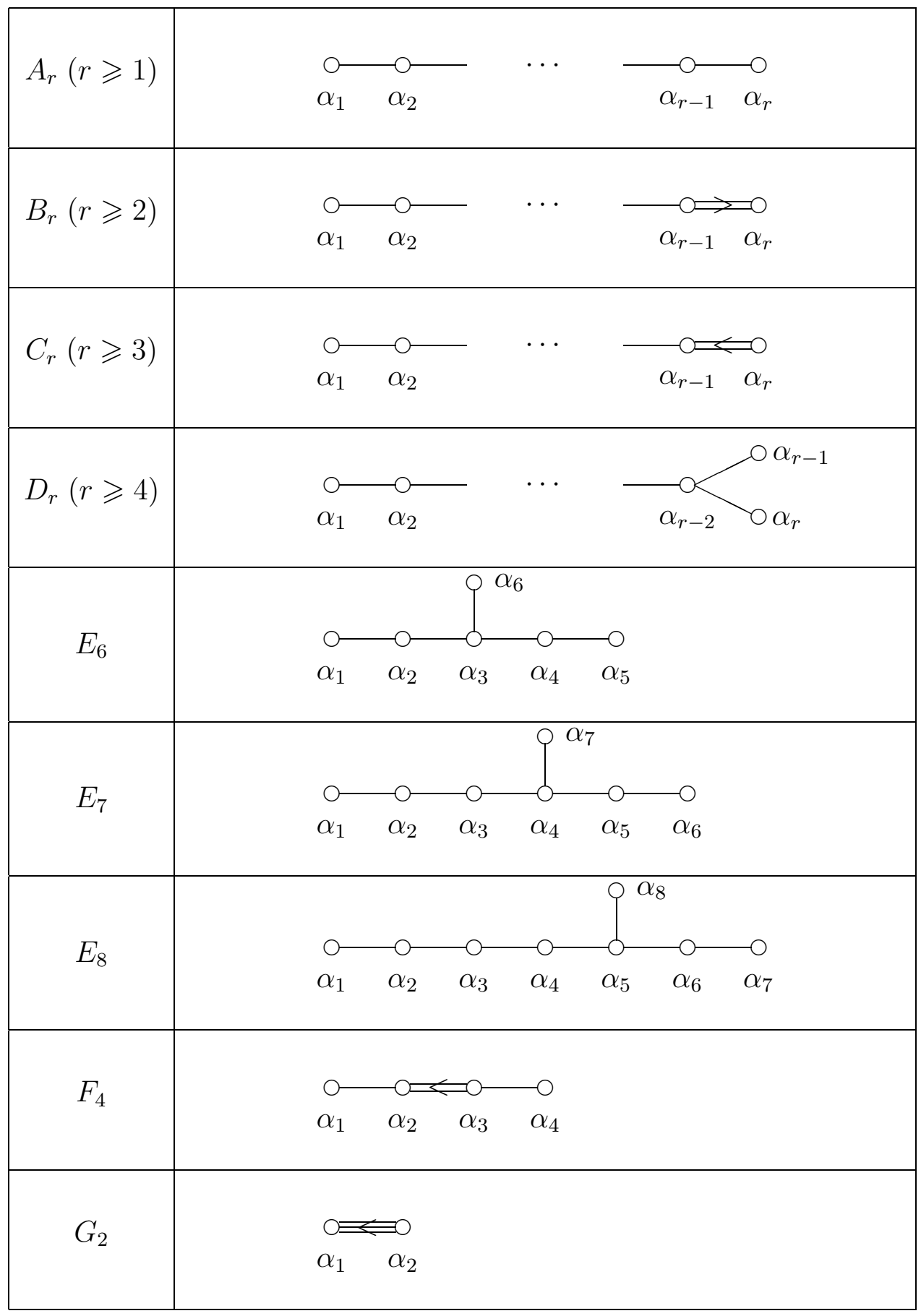

Tabela 1.1: Numeração das raizes simples no diagrama de Dynkin. 
não-negativos: tais elementos $\vartheta \in \mathfrak{h}^{*}$ são chamados pesos dominantes integrais. Então se $\rho: \mathfrak{g} \rightarrow \mathfrak{g l}(V)$ é uma representação irredutível de $\mathfrak{g}$ com peso máximo $\vartheta$, denotaremos tal representação pela $r$-upla das coordenadas de $\vartheta$ em relação à base de pesos fundamentais

$$
\rho \equiv\left(p_{1}, p_{2}, \ldots, p_{r}\right) .
$$

Suponhamos agora que $\mathfrak{g}$ é semisimples, com decomposição em ideais simples $\mathfrak{g}=\mathfrak{g}_{1} \oplus \ldots \oplus \mathfrak{g}_{k}$ tal que $\operatorname{rkg}_{j}=r_{j}(j=1, \ldots, k) ;$, então se $\rho: \mathfrak{g} \rightarrow \mathfrak{g l}(V)$ é uma representação irredutível de $\mathfrak{g}$, existem representações irredutíveis $\rho_{j}: \mathfrak{g}_{j} \rightarrow \mathfrak{g l}\left(V_{j}\right)$ dos ideais $\mathfrak{g}_{j}(j=1, \ldots, k)$, únicamente determinadas por $\rho$, tais que

$$
\rho=\bigotimes_{j=1}^{k} \rho_{j}, \quad V=\bigotimes_{j=1}^{k} V_{j},
$$

isto é, se para $X \in \mathfrak{g}$ escrevemos $X=X_{1}+\cdots+X_{k} \operatorname{com} X_{j} \in \mathfrak{g}_{j}$, então

$$
\rho(X)=\rho_{1}\left(X_{1}\right) \otimes \cdots \otimes \rho_{k}\left(X_{k}\right) .
$$

Desta forma, as representações $\rho_{j}$ determinam completamente a representação $\rho$, e se $\vartheta^{(j)}$ são os respectivos pesos máximos das representações $\rho_{j}$, podemos escrever $\rho_{j} \equiv\left(p_{1}^{(j)}, \ldots, p_{r_{j}}^{(j)}\right)$ $(j=1, \ldots, k)$, obtendo

$$
\rho \equiv\left(p_{1}^{(1)}, \ldots, p_{r_{1}}^{(1)} ; \ldots ; p_{1}^{(k)}, \ldots, p_{r_{k}}^{(k)}\right)
$$

Finalmente, se $\rho$ é uma representação completamente redutível, com decomposição

$$
\rho=\bigoplus_{\ell=1}^{n} c_{\ell} \pi_{\ell}
$$

então cada representação $\pi_{\ell}$ se decompõe num produto tensorial de representações irreduíveis dos ideais simples de $\mathfrak{g}$ conforme

$$
\pi_{\ell}=\bigotimes_{j=1}^{k}\left(\pi_{\ell}\right)^{(j)}
$$

e podemos escrever $\left(\pi_{\ell}\right)^{(j)} \equiv\left(\left(p_{\ell}\right)_{1}^{(j)}, \ldots,\left(p_{\ell}\right)_{r_{j}}^{(j)}\right)(j=1, \ldots, k)$, obtendo

$$
\pi_{\ell} \equiv\left(\left(p_{\ell}\right)_{1}^{(1)}, \ldots,\left(p_{\ell}\right)_{r_{1}}^{(1)} ; \ldots ;\left(p_{\ell}\right)_{1}^{(k)}, \ldots,\left(p_{\ell}\right)_{r_{k}}^{(k)}\right) .
$$

Juntando tudo podemos escrever:

$$
\rho \equiv\left\{\begin{array}{c}
c_{1}\left(\left(p_{1}\right)_{1}^{(1)}, \ldots,\left(p_{1}\right)_{r_{1}}^{(1)} ; \ldots ;\left(p_{1}\right)_{1}^{(k)}, \ldots,\left(p_{1}\right)_{r_{k}}^{(k)}\right) \\
\vdots \\
c_{n}\left(\left(p_{n}\right)_{1}^{(1)}, \ldots,\left(p_{n}\right)_{r_{1}}^{(1)} ; \ldots ;\left(p_{n}\right)_{1}^{(k)}, \ldots,\left(p_{n}\right)_{r_{k}}^{(k)}\right)
\end{array}\right.
$$


Portanto, se $\left\{\left(\pi_{\ell}, c_{\ell}\right) \mid \ell=1, \ldots, n\right\}$ é a regra de ramificação de uma representação irredutível de uma álgebra simples $\mathfrak{g}$ com peso máximo $\left(p_{1}, \ldots, p_{r}\right)$ em relação a uma subalgebra semisimples $\mathfrak{f}$, podemos indicar esta regra de ramificação através de

$$
\begin{gathered}
\mathfrak{g} \\
\left(p_{1}, \ldots, p_{r}\right) \rightsquigarrow c_{1}\left(\left(p_{1}\right)_{1}^{(1)}, \ldots,\left(p_{1}\right)_{r_{1}}^{(1)} ; \ldots ;\left(p_{1}\right)_{1}^{(k)}, \ldots,\left(p_{1}\right)_{r_{k}}^{(k)}\right) \\
\\
\quad c_{n}\left(\left(p_{n}\right)_{1}^{(1)}, \ldots,\left(p_{n}\right)_{r_{1}}^{(1)} ; \ldots ;\left(p_{n}\right)_{1}^{(k)}, \ldots,\left(p_{n}\right)_{r_{k}}^{(k)}\right)
\end{gathered}
$$

A notação parece pesada, mas quando escrevemos os pesos das representações explicitamente, os índices desaparecem e a notação fica muito clara.

Exemplo. Considere $\mathfrak{g}=\mathfrak{s p}(6, \mathbb{C})$ e $\rho$ a representação com peso máximo $(1,1,0)$. Então a regra de ramificação de $\rho$ com respeito à subalgebra $\mathfrak{s p}(4, \mathbb{C}) \oplus \mathfrak{s l}(2, \mathbb{C})$ é

$$
\begin{aligned}
& \mathfrak{s p}(6, \mathbb{C}) \quad \mathfrak{s p}(4, \mathbb{C}) \oplus \mathfrak{s l}(2, \mathbb{C}) \\
& (1,1,0) \quad \rightsquigarrow \quad(2,0 ; 1) \\
& (1,1 ; 0) \\
& (1,0 ; 1) \\
& (0,1 ; 1) \\
& (1,0 ; 0) \\
& (0,0 ; 1)
\end{aligned}
$$

Finalmente, há um caso especial que merece um comentário: o da álgebra $\mathfrak{s l}(2, \mathbb{C})$, que corresponde ao tipo $A_{1}$ na notação de Cartan. Esta álgebra tem posto 1 e portanto seus pesos máximos são simplesmente números inteiros não-negativos. Como $\mathfrak{s l}(2, \mathbb{C})$ tem uma única representação irredutível em cada dimensão, o conjunto dos pesos máximos é o conjunto dos números naturais, e a dimensão da representação de peso $n$ é simplesmente $n+1$. Mas existe uma convenção, usada principalmente pelos físicos, que consiste em indexar as representações irredutíveis de $\mathfrak{s l}(2, \mathbb{C})$ por números semi-inteiros não-negativos: este número é chamado de spin da representação e é igual a $\frac{n}{2}$; isto é, a dimensão da representação irredutível de spin $s$ de $\mathfrak{s l}(2, \mathbb{C})$ é $2 s+1$ e seu peso máximo é $2 s\left(s=0, \frac{1}{2}, 1, \ldots\right)$.

\subsection{Estrutura das Subalgebras Maximais das Álgebras de Lie Semisimples}

Nesta seção provaremos teoremas que reduzem o problema da classificação das subalgebras maximais de uma álgebra de Lie semisimples à classificação das subalgebras maximais de uma álgebras de Lie simples. 
Primeiramente daremos uma definição de subalgebra maximal, que é a mais conveniente para as demonstrações que faremos.

Definição 1.2.1. Seja $\mathfrak{g}$ uma álgebra de Lie. Uma subalgebra $\mathfrak{h} \subset \mathfrak{g}$ é dita maximal se $\mathfrak{h} \neq \mathfrak{g}$ e para toda subalgebra $\mathfrak{f}$ tal que $\mathfrak{h} \subset \mathfrak{f} \subset \mathfrak{g}$ temos que $\mathfrak{f}=\mathfrak{h}$ ou $\mathfrak{f}=\mathfrak{g}$.

Para provar o teorema principal precisaremos de um lema que foi provado pela primeira vez por Morozov $^{1}$ e depois reproduzido por Golubitsky [16], que é fundamental para a demonstração que daremos para o teorema de Dynkin.

Lema 1.2.1 (Morozov-Golubitsky). Sejam $\mathfrak{g}$ uma álgebra de Lie semisimples e não simples e $\mathfrak{h}$ uma subalgebra maximal de $\mathfrak{g}$ tal que $\mathfrak{h}$ não contém nenhum ideal não-trivial de $\mathfrak{g}$. Então existe uma álgebra de Lie simples $\mathfrak{g}_{0}$ tal que $\mathfrak{g} \cong \mathfrak{g}_{0} \oplus \mathfrak{g}_{0}$ e $\mathfrak{h} \cong \mathfrak{g}_{0}$ com a inclusão diagonal: $\mathfrak{h}=\left\{(X, X) \mid X \in \mathfrak{g}_{0}\right\}$.

DemonstraÇÃo. Seja $\mathfrak{g}_{0}$ um ideal próprio não-trivial de $\mathfrak{g}$ e $\mathfrak{g}_{0}^{\prime}$ o ideal complementar. (Isto significa que $\mathfrak{g}_{0}$ é uma soma direta qualquer dos ideais simples de $\mathfrak{g}$ e $\mathfrak{g}_{0}^{\prime}$ é a soma direta dos demais, ambos não-triviais.) Então $\mathfrak{h} \not \subset \mathfrak{g}_{0}, \mathfrak{h} \not \subset \mathfrak{g}_{0}^{\prime}$ e isto implica que $\mathfrak{h}+\mathfrak{g}_{0}$ e $\mathfrak{h}+\mathfrak{g}_{0}^{\prime}$ são subalgebras de $\mathfrak{g}$ que contêm $\mathfrak{h}$ no sentido estrito, $\mathfrak{h} \varsubsetneqq \mathfrak{h}+\mathfrak{g}_{0}$ e $\mathfrak{h} \varsubsetneqq \mathfrak{h}+\mathfrak{g}_{0}^{\prime}$; portanto, pela maximalidade de $\mathfrak{h}, \mathfrak{g}=\mathfrak{h}+\mathfrak{g}_{0}$ e $\mathfrak{g}=\mathfrak{h}+\mathfrak{g}_{0}^{\prime}$. Por outro lado, $\left[\mathfrak{h} \cap \mathfrak{g}_{0}, \mathfrak{h}\right] \subset \mathfrak{h} \cap \mathfrak{g}_{0}$ e $\left[\mathfrak{h} \cap \mathfrak{g}_{0}, \mathfrak{g}_{0}^{\prime}\right]=\{0\} ;$ portanto, $\mathfrak{h} \cap \mathfrak{g}_{0}$ é um ideal em $\mathfrak{g}$, obviamente contido em $\mathfrak{h}$, o que pela hipótese implica $\mathfrak{h} \cap \mathfrak{g}_{0}=\{0\}$ e, finalmente,

$$
\mathfrak{g}=\mathfrak{h} \oplus \mathfrak{g}_{0}
$$

Como $\mathfrak{g}_{0}$ era um ideal próprio não-trivial de $\mathfrak{g}$ qualquer, isto significa que todos os ideais próprios não-triviais de $\mathfrak{g}$ têm a mesma dimensão. Isso é possível apenas no caso em que $\mathfrak{g}$ é a soma direta de exatamente dois ideais simples $\mathfrak{g}_{1}$ e $\mathfrak{g}_{2}$ da mesma dimensão,

$$
\mathfrak{g}=\mathfrak{g}_{1} \oplus \mathfrak{g}_{2}
$$

tal que

$$
\mathfrak{g}=\mathfrak{h} \oplus \mathfrak{g}_{1}=\mathfrak{h} \oplus \mathfrak{g}_{2}
$$

O próximo passo consiste em provar que $\mathfrak{g}_{1} \cong \mathfrak{g}_{2}$, não apenas como espaços vetoriais mas também como álgebras de Lie. Para $i=1,2$, introduzimos as projeções

$$
\begin{array}{ccc}
\pi_{i}: \quad \mathfrak{g}=\mathfrak{g}_{1} \oplus \mathfrak{g}_{2} & \longrightarrow & \mathfrak{g}_{i} \\
X & \mapsto & X_{i}
\end{array}
$$

\footnotetext{
${ }^{1}$ Em sua tese de doutorado On Nonsemisimple Maximal Subgroups of Simple Groups, apresentada em 1943 na Universidade de Kazan. Infelizmente só existe a versão em russo deste trabalho.
} 
de $\mathfrak{g}$ sobre $\mathfrak{g}_{i}$ que são homomorfismos de álgebras de Lie devido ao fato de que os $\mathfrak{g}_{i}$ são ideais em g. Para construir isomorfismos explícitos $P_{12}: \mathfrak{g}_{1} \rightarrow \mathfrak{g}_{2}$ e $P_{21}: \mathfrak{g}_{2} \rightarrow \mathfrak{g}_{1}$, observamos que a restrição de $\pi_{i}$ à subalgebra $\mathfrak{h}$ de $\mathfrak{g}$ é sobrejetora e injetora. De fato, se tivessimos $\pi_{i}(\mathfrak{h}) \varsubsetneqq \mathfrak{g}_{i}$, existiria uma subalgebra maximal $\tilde{\mathfrak{g}}_{i}$ de $\mathfrak{g}_{i}$ tal que $\pi_{i}(\mathfrak{h}) \subset \tilde{\mathfrak{g}}_{i}$, e então a subalgebra $\tilde{\mathfrak{g}}=\tilde{\mathfrak{g}}_{1} \oplus \mathfrak{g}_{2}($ para $i=1)$ ou $\tilde{\mathfrak{g}}=\mathfrak{g}_{1} \oplus \tilde{\mathfrak{g}}_{2}$ (para $\left.i=2\right)$ claramente conteria $\mathfrak{h}$ e seria diferente de $\mathfrak{g}$, o que contradiria a maximalidade de $\mathfrak{h}$, mostrando assim que a projeção $\pi_{i}$ é sobrejetora. Por outro lado, se $X \in \mathfrak{h}$ satisfaz $\pi_{i}(X)=0$, então $X \in \mathfrak{h} \cap \mathfrak{g}_{2}=\{0\}$ (para $i=1$ ) ou $X \in \mathfrak{h} \cap \mathfrak{g}_{1}=\{0\}$ (para $i=2$ ), mostrando assim que a projeção $\pi_{i}$ é injetora. Logo, $\mathfrak{g}_{1} \cong \mathfrak{h} \cong \mathfrak{g}_{2}$ e $\mathfrak{g} \cong \mathfrak{g}_{1} \oplus \mathfrak{g}_{2} \cong \mathfrak{h} \oplus \mathfrak{h}$.

O proximo teorema, que se encontra em Dynkin [10, pag. 235, teo. 15.1], é o principal teorema desta seção.

Teorema 1.2.1 (Dynkin [10]). Seja $\mathfrak{g}$ uma álgebra de Lie semisimples e $\mathfrak{g}=\mathfrak{g}_{1} \oplus \ldots \oplus \mathfrak{g}_{r}$ sua decomposição em soma direta de ideais simples. As subalgebras maximais $\tilde{\mathfrak{g}}$ de $\mathfrak{g}$ são de dois tipos:

1. Tipo simples:

$\widetilde{\mathfrak{g}}=\mathfrak{g}_{1} \oplus \ldots \oplus \widetilde{\mathfrak{g}}_{i} \oplus \ldots \oplus \mathfrak{g}_{r} \quad(1 \leqslant i \leqslant r)$, onde $\widetilde{\mathfrak{g}}_{i}$ é subalgebra maximal de $\mathfrak{g}_{i} ;$

2. Tipo diagonal:

$\widetilde{\mathfrak{g}}=\mathfrak{g}_{1} \oplus \ldots \oplus \mathfrak{g}_{i-1} \oplus \mathfrak{g}_{i+1} \oplus \ldots \oplus \mathfrak{g}_{j-1} \oplus \mathfrak{g}_{j+1} \oplus \ldots \oplus \mathfrak{g}_{r} \oplus \mathfrak{g}_{i j} \quad(1 \leqslant i<j \leqslant r)$, onde $\mathfrak{g}_{i}$ e $\mathfrak{g}_{j}$ são ideais simples isomorfos de $\mathfrak{g}, P_{i j}: \mathfrak{g}_{i} \rightarrow \mathfrak{g}_{j}$ é algum isomorfismo e $\mathfrak{g}_{i j}=\left\{\left(X, P_{i j} X\right) \in \mathfrak{g}_{i} \oplus \mathfrak{g}_{j} \mid X \in \mathfrak{g}_{i}\right\}$.

Observação. A existência de subalgebras maximais do tipo diagonal requer que pelo menos dois dos ideais simples $\mathfrak{g}_{1}, \ldots, \mathfrak{g}_{\text {r }}$ de $\mathfrak{g}$ sejam iguais (a menos de um isomorfismo).

Demonstração. Pela decomposição em soma direta, todo elemento $X \in \mathfrak{g}$ pode ser escrito da seguinte forma:

$$
X=X_{1}+\ldots+X_{r} \quad \operatorname{com} \quad X_{i} \in \mathfrak{g}_{i} .
$$

Além disso, como esta representação é única, cada $X_{i}$ é determinado por $X$. Assim definimos as projeções sobre cada fator $\mathfrak{g}_{i}$ por

$$
\begin{aligned}
& \pi_{i}: \mathfrak{g} \longrightarrow \mathfrak{g}_{i} \\
& X \mapsto X_{i}
\end{aligned}
$$

que são homomorfismos de álgebras de Lie devido ao fato de que os $\mathfrak{g}_{i}$ são ideais em $\mathfrak{g}$.

Seja então $\mathfrak{h}$ uma subalgebra maximal de $\mathfrak{g}$.

Consideramos primeiro o caso em que $\pi_{i}(\mathfrak{h}) \varsubsetneqq \mathfrak{g}_{i}$ para ao menos um $i$. Seja $\widetilde{\mathfrak{g}}_{i}$ uma subalgebra maximal de $\mathfrak{g}_{i}$ tal que $\pi_{i}(\mathfrak{h}) \subset \widetilde{\mathfrak{g}}_{i}$. A subalgebra $\widetilde{\mathfrak{g}}=\mathfrak{g}_{1} \oplus \ldots \oplus \widetilde{\mathfrak{g}}_{i} \oplus \ldots \oplus \mathfrak{g}_{r}$ 
claramente contém $\mathfrak{h}$ e é diferente de $\mathfrak{g}$, mas como $\mathfrak{h}$ é maximal, temos que $\mathfrak{h}=\widetilde{\mathfrak{g}}$. Agora consideraremos o caso em que

$$
\pi_{k}(\mathfrak{h})=\mathfrak{g}_{k} \quad \text { para } \quad k=1, \ldots, r .
$$

Mostraremos primeiro que para $k=1,2, \ldots, r, \mathfrak{g}_{k} \cap \mathfrak{h}$ é um ideal em $\mathfrak{g}_{k}$. De fato, dados $Y \in$ $\mathfrak{g}_{k} \cap \mathfrak{h}$ e $Z \in \mathfrak{g}_{k}$, podemos escolher $X \in \mathfrak{h}$ tal que $\pi_{k}(X)=Z$ e, escrevendo $X=X_{1}+\ldots+X_{r}$, temos $X_{k}=Z$ e $\left[Y, X_{l}\right] \in \mathfrak{g}_{k} \cap \mathfrak{g}_{l}=\{0\}$ se $l \neq k ; \operatorname{logo}[Y, X]=\left[Y, X_{1}+\ldots+X_{r}\right]=\left[Y, X_{k}\right]=$ $[Y, Z]$ e portanto $[Y, Z]=[Y, X] \in \mathfrak{g}_{k} \cap \mathfrak{h}$. Como $\mathfrak{g}_{k}$ é simples, devemos ter

$$
\mathfrak{g}_{k} \cap \mathfrak{h}=\mathfrak{g}_{k}, \quad \text { i.e. } \quad \mathfrak{g}_{k} \subset \mathfrak{h}, \quad \text { ou } \quad \mathfrak{g}_{k} \cap \mathfrak{h}=\{0\} .
$$

Em geral, sejam

$$
\mathfrak{g}^{\prime}=\bigoplus_{\mathfrak{g}_{k} \cap \mathfrak{h}=\{0\}} \mathfrak{g}_{k} \quad \text { e } \quad \mathfrak{g}^{\prime \prime}=\bigoplus_{\mathfrak{g}_{k} \subset \mathfrak{h}} \mathfrak{g}_{k} .
$$

Obviamente, temos $\mathfrak{g}^{\prime \prime} \subset \mathfrak{h}$ e, portanto, pondo $\mathfrak{h}^{\prime}=\mathfrak{g}^{\prime} \cap \mathfrak{h}$, podemos escrever $\mathfrak{h}=\mathfrak{h}^{\prime} \oplus \mathfrak{g}^{\prime \prime}$. Observamos também que o conjunto de índices $k$ para os quais $\mathfrak{g}_{k} \cap \mathfrak{h}=\{0\}$ não é vazio (pois $\mathfrak{h} \neq \mathfrak{g}$ ) e contém pelo menos dois elementos (se tivessemos $\mathfrak{g}_{k} \cap \mathfrak{h}=\{0\}$ para um único índice $k$, poderiamos concluir que $\mathfrak{g}_{1} \oplus \ldots \oplus \mathfrak{g}_{k-1} \oplus \mathfrak{g}_{k+1} \oplus \ldots \oplus \mathfrak{g}_{r} \subset \mathfrak{h}$, e como $\pi_{k}(\mathfrak{h})=\mathfrak{g}_{k}$, teriamos também que $\mathfrak{g}_{k} \subset \mathfrak{h}$, o que é absurdo). Portanto, a álgebra de Lie $\mathfrak{g}^{\prime}$ é semisimples e não é simples, e $\mathfrak{h}^{\prime}$ é uma subálgebra maximal de $\mathfrak{g}^{\prime}$ que não contém nenhum ideal próprio de $\mathfrak{g}^{\prime}$. Aplicação do lema 1.2.1 termina a demonstração.

Este teorema fornece um procedimento para se determinar todas as subalgebras maximais semisimples de uma álgebra de Lie semisimples, desde que se tenha uma classificação de todas as subalgebras maximais semisimples das álgebras de Lie simples.

Agora vamos determinar as classes de conjugação destas subalgebras. Para tanto, precisamos do seguinte lema.

Lema 1.2.2. Seja $\mathfrak{g}$ uma álgebra de Lie semisimples $e \mathfrak{g}=\mathfrak{g}_{1} \oplus \ldots \oplus \mathfrak{g}_{r}$ sua decomposição em soma direta de ideais simples. Então

$$
\operatorname{Int}(\mathfrak{g})=\operatorname{Int}\left(\mathfrak{g}_{1}\right) \times \ldots \times \operatorname{Int}\left(\mathfrak{g}_{r}\right)
$$

DemonstraÇÃo. Seja $\sigma$ um automorfismo interno de $\mathfrak{g}$; estudaremos primeiro o caso em que $\sigma$ é da forma exp ad $(X)$ para $X \in \mathfrak{g}$. Vamos escrever $X=X_{1}+\ldots+X_{r} \operatorname{com} X_{i} \in \mathfrak{g}_{i}$, então é claro que $\operatorname{ad}\left(X_{i}\right) \operatorname{ad}\left(X_{j}\right)=\operatorname{ad}\left(X_{j}\right) \operatorname{ad}\left(X_{i}\right)$, para $i, j$ quaisquer. Portanto para todo $X \in \mathfrak{g}$ temos:

$$
\begin{aligned}
\exp \operatorname{ad}(X) & =\exp \operatorname{ad}\left(X_{1}+\ldots+X_{r}\right) \\
& =\exp \left(\operatorname{ad}\left(X_{1}\right)+\ldots+\operatorname{ad}\left(X_{r}\right)\right) \\
& =\exp \operatorname{ad}\left(X_{1}\right) \cdot \ldots \cdot \exp \operatorname{ad}\left(X_{r}\right) .
\end{aligned}
$$


Escrevendo $Y=Y_{1}+\cdots+Y_{r} \operatorname{com} Y_{j} \in \mathfrak{g}_{j}$, temos $\exp \operatorname{ad}\left(X_{i}\right)\left(Y_{j}\right)=Y_{j}$ para $i \neq j$ e portanto

$$
\begin{aligned}
\exp \operatorname{ad}\left(X_{i}\right)(Y) & =\exp \operatorname{ad}\left(X_{i}\right)\left(Y_{1}+\ldots+Y_{r}\right) \\
& =\exp \operatorname{ad}\left(X_{i}\right)\left(Y_{1}\right)+\ldots+\exp \operatorname{ad}\left(X_{i}\right)\left(Y_{r}\right) \\
& =Y_{1}+\ldots+Y_{i-1}+\exp \operatorname{ad}\left(X_{i}\right)\left(Y_{i}\right)+Y_{i+1}+\ldots+Y_{r}
\end{aligned}
$$

Daí,

$$
\exp \operatorname{ad}(X)(Y)=\prod_{i=1}^{r} \exp \operatorname{ad}\left(X_{i}\right)\left(Y_{i}\right)
$$

Isto mostra que para todo $i=1, \ldots, r, \sigma\left(\mathfrak{g}_{i}\right) \subset \mathfrak{g}_{i}$ e $\sigma$ restrito a $\mathfrak{g}_{i}$ coincide com $\exp \operatorname{ad}\left(X_{i}\right)$. Agora, escrevendo $\sigma_{i}=\exp \operatorname{ad}\left(X_{i}\right)$, que é um automorfismo interno de $\mathfrak{g}_{i}$, temos que $\sigma=$ $\sigma_{1} \cdot \ldots \cdot \sigma_{r} \operatorname{com} \sigma_{i} \sigma_{j}=\sigma_{j} \sigma_{i}$ se $i \neq j$.

Passando ao caso geral, seja $\sigma \in \operatorname{Int}(\mathfrak{g})$ qualquer, então $\sigma=\sigma_{1} \cdot \ldots \cdot \sigma_{k}$, onde cada $\sigma_{i}$ é da forma exp $\operatorname{ad}(X) \operatorname{com} X \in \mathfrak{g}$. Então podemos escrever cada $\sigma_{i}$ como produto de automorfismos internos de cada ideal de $\mathfrak{g}$, como automorfismos que correspondem a ideais diferentes comutam, podemos escrever o automorfismo interno $\sigma$ de $\mathfrak{g}$ como um produto de automorfismos internos de seus ideais $\mathfrak{g}_{1}, \ldots, \mathfrak{g}_{r}$. Com isso, mostramos que

$$
\operatorname{Int}(\mathfrak{g})=\operatorname{Int}\left(\mathfrak{g}_{1}\right) \cdot \ldots \cdot \operatorname{Int}\left(\mathfrak{g}_{r}\right)
$$

Para mostrar que o produto é direto precisamos provar que

$$
\operatorname{Int}\left(\mathfrak{g}_{i}\right) \cap\left(\operatorname{Int}\left(\mathfrak{g}_{i+1}\right) \cdot \ldots \cdot \operatorname{Int}\left(\mathfrak{g}_{r}\right)\right)=\{1\}
$$

para todo $i=1, \ldots, r$. Mas isto decorre do fato de que o centro do grupo de automorfismos internos de uma álgebra de Lie semisimples é trivial [19, pag.129, cor. 5.3].

Agora podemos demonstrar o teorema de conjugação, que reduz o problema de decidir se duas subalgebras maximais de uma álgebra de Lie semisimples $\mathfrak{g}$ são conjugadas ao caso em que $\mathfrak{g}$ é simples. Este teorema aparece junto com o teorema 1.2.1 na versão original de Dynkin [10].

Teorema 1.2.2 (Dynkin [10]). Seja $\mathfrak{g}$ uma álgebra de Lie semisimples e $\mathfrak{g}=\mathfrak{g}_{1} \oplus \cdots \oplus \mathfrak{g}_{r}$ sua decomposição em soma direta de ideais simples. Seja $\mathfrak{h}$ uma subalgebra maximal de $\mathfrak{g}$. Se $\mathfrak{h}$ for do tipo simples vamos escrever $\mathfrak{h}=\mathfrak{h}\left(i, \widetilde{\mathfrak{g}}_{i}\right)$ com

$$
\mathfrak{h}\left(i, \widetilde{\mathfrak{g}}_{i}\right)=\bigoplus_{\substack{k=1 \\ k \neq i}}^{r} \mathfrak{g}_{k} \oplus \widetilde{\mathfrak{g}}_{i}
$$


com $\tilde{\mathfrak{g}}_{i}$ subalgebra maximal de $\mathfrak{g}_{i}$ e se $\mathfrak{h}$ for do tipo diagonal vamos escrever $\mathfrak{h}=\mathfrak{h}\left(i, j, P_{i j}\right)$ com

$$
\mathfrak{h}\left(i, j, P_{i j}\right)=\bigoplus_{\substack{k=1 \\ k \neq i, j}}^{r} \mathfrak{g}_{k} \oplus \mathfrak{g}_{i j}
$$

onde $i \neq j$ e $\mathfrak{g}_{i j}=\left\{\left(X_{i}, P_{i j}\left(X_{i}\right)\right) \mid X_{i} \in \mathfrak{g}_{i}\right\}$ para algum isomorfismo $P_{i j}: \mathfrak{g}_{i} \rightarrow \mathfrak{g}_{j}$. Então:

1. A condição necessária e suficiente para que $\mathfrak{h}\left(i_{1}, \widetilde{\mathfrak{g}}_{i_{1}}\right)$ e $\mathfrak{h}\left(i_{2}, \widetilde{\mathfrak{g}}_{i_{2}}\right)$ sejam conjugadas em $\mathfrak{g}$ é que $i_{1}=i_{2}$ e que $\widetilde{\mathfrak{g}}_{i_{1}}$ e $\widetilde{\mathfrak{g}}_{i_{2}}$ sejam conjugadas em $\mathfrak{g}_{i_{1}}=\mathfrak{g}_{i_{2}}$.

2. A condição necessária e suficiente para que $\mathfrak{h}\left(i_{1}, j_{1}, P_{i_{1} j_{1}}\right)$ e $\mathfrak{h}\left(i_{2}, j_{2}, P_{i_{2} j_{2}}\right)$ sejam conjugadas em $\mathfrak{g}$ é que $i_{1}=i_{2}, j_{1}=j_{2}$ e que $P_{i_{1} j_{1}}$ seja levado em $P_{i_{2} j_{2}}$ por composição com automorfismos internos de $\mathfrak{g}_{i_{1}}=\mathfrak{g}_{i_{2}}$ e de $\mathfrak{g}_{j_{1}}=\mathfrak{g}_{j_{2}}$.

Além disto $\mathfrak{h}\left(i, \widetilde{\mathfrak{g}}_{i}\right)$ e $\mathfrak{h}\left(i, j, P_{i j}\right)$ nunca são conjugadas.

DemonstraÇÃo. Observe que o lema 1.2.2 mostra que os automorfismos internos de $\mathfrak{g}$ sempre preservam a decomposição direta de $\mathfrak{g}$ em ideais simples: automorfismos que induzem permutações entre ideais simples isomorfos são necessariamente externos. Além disto, como $\mathfrak{h}\left(j, i, P_{j i}\right)=\mathfrak{h}\left(i, j, P_{i j}\right)$ com $P_{i j}=P_{j i}^{-1}$ podemos supor desde já que $i<j$.

Mostraremos primeiro a afirmação 1. Obviamente, se $i_{1}=i_{2}$ e se $\widetilde{\mathfrak{g}}_{i_{1}}$ e $\widetilde{\mathfrak{g}}_{i_{2}}$ são conjugadas em $\mathfrak{g}_{i_{1}}=\mathfrak{g}_{i_{2}}$, basta extender o automorfismo interno de $\mathfrak{g}_{i_{1}}=\mathfrak{g}_{i_{2}}$ que leva $\widetilde{\mathfrak{g}}_{i_{1}}$ para $\widetilde{\mathfrak{g}}_{i_{2}}$ pela identidade nos demais ideais simples de $\mathfrak{g}$ para obter um automorfismo interno de $\mathfrak{g}$ que leva $\mathfrak{h}\left(i_{1}, \widetilde{\mathfrak{g}}_{i_{1}}\right)$ para $\mathfrak{h}\left(i_{2}, \widetilde{\mathfrak{g}}_{i_{2}}\right)$. Reciprocamente, suponhamos que $\mathfrak{h}\left(i_{1}, \widetilde{\mathfrak{g}}_{i_{1}}\right)$ e $\mathfrak{h}\left(i_{2}, \widetilde{\mathfrak{g}}_{i_{2}}\right)$ são conjugadas em $\mathfrak{g}$, isto é, existe um automorfismo interno $\sigma$ de $\mathfrak{g}$ tal que $\sigma\left(\mathfrak{h}\left(i_{1}, \widetilde{\mathfrak{g}}_{i_{1}}\right)\right)=\mathfrak{h}\left(i_{1}, \widetilde{\mathfrak{g}}_{i_{2}}\right)$. Pelo lema 1.2.2 podemos escrever $\sigma=\sigma_{1} \cdot \ldots \cdot \sigma_{r}$ com automorfismos internos $\sigma_{\ell}$ de $\mathfrak{g}_{\ell}(\ell=1, \ldots, r)$ e usando a decomposição (1.2), obtemos

$$
\mathfrak{h}\left(i_{2}, \widetilde{\mathfrak{g}}_{i_{2}}\right)=\bigoplus_{\substack{k=1 \\ k \neq i_{2}}}^{r} \mathfrak{g}_{k} \oplus \widetilde{\mathfrak{g}}_{i_{2}}
$$

e por outro lado

$$
\begin{aligned}
\mathfrak{h}\left(i_{2}, \widetilde{\mathfrak{g}}_{i_{2}}\right) & =\sigma\left(\mathfrak{h}\left(i_{1}, \widetilde{\mathfrak{g}}_{i_{1}}\right)\right) \\
& =\left(\sigma_{1} \cdot \ldots \cdot \sigma_{r}\right)\left(\mathfrak{h}\left(i_{1}, \widetilde{\mathfrak{g}}_{i_{1}}\right)\right) \\
& =\bigoplus_{\substack{k=1 \\
k \neq i_{1}}}^{r} \mathfrak{g}_{k} \oplus \sigma_{i_{1}}\left(\widetilde{\mathfrak{g}}_{i_{1}}\right)
\end{aligned}
$$


o que, pela unicidade da decomposição (1.2) e pelo fato de que $\widetilde{\mathfrak{g}}_{i_{1}} \varsubsetneqq \mathfrak{g}_{i_{1}}$ e $\widetilde{\mathfrak{g}}_{i_{2}} \varsubsetneqq \mathfrak{g}_{i_{2}}$, implica que $i_{1}=i_{2}$ e $\sigma_{i_{1}}\left(\widetilde{\mathfrak{g}}_{i_{1}}\right)=\widetilde{\mathfrak{g}}_{i_{2}}$.

Antes de mostrar a afirmação 2 faremos algumas observações. Suponhamos que $\mathfrak{g}_{i}$ e $\mathfrak{g}_{j}$ são dois ideais isomorfos de $\mathfrak{g}$ e, dado um isomorfismo $P_{i j}: \mathfrak{g}_{i} \rightarrow \mathfrak{g}_{j}$, consideremos a aplicação $\psi_{i j}: \mathfrak{g} \rightarrow \mathfrak{g}$ definida por

$$
\psi_{i j}\left(X^{\prime}+X_{i}+X_{j}\right)=X^{\prime}+\left(P_{i j}\right)^{-1}\left(X_{j}\right)+P_{i j}\left(X_{i}\right)
$$

onde $X=X^{\prime}+X_{i}+X_{j}$ é a decomposição direta de $X \in \mathfrak{g}$ induzida pela decomposição direta $\mathfrak{g}=\mathfrak{g}^{\prime} \oplus \mathfrak{g}_{i} \oplus \mathfrak{g}_{j}, \mathrm{com}$

$$
\mathfrak{g}^{\prime}=\bigoplus_{\substack{k=1 \\ k \neq i, j}}^{r} \mathfrak{g}_{k}
$$

É claro que $\psi_{i j}$ é um automorfismo de $\mathfrak{g}$ e que a subalgebra $\mathfrak{h}\left(i, j, P_{i j}\right)=\mathfrak{g}^{\prime} \oplus \mathfrak{g}_{i j} \operatorname{com} \mathfrak{g}_{i j}=$ $\left\{\left(X_{i}, P_{i j}\left(X_{i}\right)\right) \mid X_{i} \in \mathfrak{g}_{i}\right\}$ é a subalgebra dos elementos fixos por $\psi_{i j}$. Como este automorfismo permuta dois ideais isomorfos o lema 1.2.2 implica que ele é um automorfismo externo de $\mathfrak{g}$. Agora suponhamos que o isomorfismo $P_{i j}^{(2)}: \mathfrak{g}_{i} \rightarrow \mathfrak{g}_{j}$ definindo $\mathfrak{g}_{i j}^{(2)}$ é obtido do isomorfismo $P_{i j}^{(1)}: \mathfrak{g}_{i} \rightarrow \mathfrak{g}_{j}$ definindo $\mathfrak{g}_{i j}^{(1)}$ pondo $P_{i j}^{(2)}=\sigma_{j} \circ P_{i j}^{(1)} \circ \sigma_{i}^{-1}$ com automorfismos internos $\sigma_{i}$ de $\mathfrak{g}_{i}$ e $\sigma_{j}$ de $\mathfrak{g}_{j}$, então se definimos o automorfismo interno $\sigma$ de $\mathfrak{g}$ pondo $\sigma=1 \oplus \sigma_{i} \oplus \sigma_{j}$ temos que $\psi_{i j}^{(2)}=\sigma \circ \psi_{i j}^{(1)} \circ \sigma^{-1}$. Por outro lado, se $\sigma$ é um automorfismo interno de $\mathfrak{g}$ tal que $\psi_{i j}^{(2)}$ e $\psi_{i j}^{(1)}$ sejam conjugados, isto é, $\psi_{i j}^{(2)}=\sigma \circ \psi_{i j}^{(1)} \circ \sigma^{-1}$ então pelo lema 1.2.2 podemos escrever $\sigma=\sigma_{1} \cdot \ldots \cdot \sigma_{r}$ com automorfismos internos $\sigma_{\ell}$ de $\mathfrak{g}_{\ell}(\ell=1, \ldots, r)$ e portanto temos que $P_{i j}^{(2)}=\sigma_{j} \circ P_{i j}^{(1)} \circ \sigma_{i}^{-1}$. Ou seja, os isomorfismos $P_{i j}^{(2)}$ e $P_{i j}^{(1)}$ são levados um no outro por automorfismos internos de $\mathfrak{g}_{i}$ e de $\mathfrak{g}_{j}$ se e somente se os automorfismos $\psi_{i j}^{(2)}$ e $\psi_{i j}^{(1)}$ de $\mathfrak{g}$ são conjugados em Autg por um automorfismo interno de $\mathfrak{g}$.

Agora passaremos a demonstração da afirmação do item 2. Obviamente, se $i_{1}=i=$ $i_{2}, j_{1}=j=j_{2}$ e se o isomorfismo $P_{i j}^{(2)}: \mathfrak{g}_{i} \rightarrow \mathfrak{g}_{j}$ definindo $\mathfrak{g}_{i j}^{(2)}$ é obtido do isomorfismo $P_{i j}^{(1)}: \mathfrak{g}_{i} \rightarrow \mathfrak{g}_{j}$ definindo $\mathfrak{g}_{i j}^{(1)}$ pondo $P_{i j}^{(2)}=\sigma_{j} \circ P_{i j}^{(1)} \circ \sigma_{i}^{-1}$ com automorfismos internos $\sigma_{i}$ de $\mathfrak{g}_{i}$ e $\sigma_{j}$ de $\mathfrak{g}_{j}$, então pelas observações acima podemos construir um automorfismo interno $\sigma$ de $\mathfrak{g}$ tal que $\psi_{i j}^{(2)}=\sigma \circ \psi_{i j}^{(1)} \circ \sigma^{-1}$ e obtemos

$$
\begin{aligned}
X \in \mathfrak{h}\left(i, j, P_{i j}^{(1)}\right) & \Longleftrightarrow \psi_{i j}^{(1)}(X)=X \\
& \Longleftrightarrow \psi_{i j}^{(2)}(\sigma(X))=\sigma\left(\psi_{i j}^{(1)}(X)\right)=\sigma(X) \\
& \Longleftrightarrow \sigma(X) \in \mathfrak{h}\left(i, j, P_{i j}^{(2)}\right)
\end{aligned}
$$

mostrando que $\sigma$ leva $\mathfrak{h}\left(i, j, P_{i j}^{(1)}\right)$ para $\mathfrak{h}\left(i, j, P_{i j}^{(2)}\right)$. Reciprocamente, suponhamos que $\mathfrak{h}\left(i_{1}, j_{1}, P_{i_{1} j_{1}}\right)$ e $\mathfrak{h}\left(i_{2}, j_{2}, P_{i_{2} j_{2}}\right)$ são conjugadas em $\mathfrak{g}$, isto é, existe um automorfismo interno $\sigma$ de $\mathfrak{g}$ tal que $\sigma\left(\mathfrak{h}\left(i_{1}, j_{1}, P_{i_{1} j_{1}}\right)\right)=\mathfrak{h}\left(i_{2}, j_{2}, P_{i_{2} j_{2}}\right)$. Aplicando o lema 1.2 .2 a $\sigma$ temos a 
decomposição $\sigma=\sigma_{1} \cdot \ldots \cdot \sigma_{r}$ com automorfismos internos $\sigma_{\ell}$ de $\mathfrak{g}_{\ell}(\ell=1, \ldots, r)$ e usando a decomposição (1.3), obtemos

$$
\mathfrak{h}\left(i_{2}, j_{2}, P_{i_{2} j_{2}}\right)=\bigoplus_{\substack{k=1 \\ k \neq i_{2}, j_{2}}}^{r} \mathfrak{g}_{k} \oplus \mathfrak{g}_{i_{2} j_{2}},
$$

e por outro lado

$$
\begin{aligned}
\mathfrak{h}\left(i_{2}, j_{2}, P_{i_{2} j_{2}}\right) & =\sigma\left(\mathfrak{h}\left(i_{1}, j_{1}, P_{i_{1} j_{1}}\right)\right) \\
& =\left(\sigma_{1} \cdot \ldots \cdot \sigma_{r}\right)\left(\mathfrak{h}\left(i_{1}, j_{1}, P_{i_{1} j_{1}}\right)\right) \\
& =\bigoplus_{\substack{k=1 \\
k \neq i_{1}, j_{1}}}^{r} \mathfrak{g}_{k} \oplus\left(\sigma_{i_{1}} \oplus \sigma_{j_{1}}\right)\left(\mathfrak{g}_{i_{1} j_{1}}\right)
\end{aligned}
$$

o que, pela unicidade da decomposição (1.2) e pelo fato de que $i_{1}<j_{1}$ e $i_{2}<j_{2}$, implica que $i_{1}=i=i_{2}$ e $j_{1}=j=j_{2}$. Portanto, podemos escrever $P_{i_{1} j_{1}}=P_{i j}^{(1)}$ e $P_{i_{2} j_{2}}=P_{i j}^{(2)}$ e obtemos

$$
\begin{aligned}
\psi_{i j}^{(1)}(X)=X & \Longleftrightarrow X \in \mathfrak{h}\left(i, j, P_{i j}^{(1)}\right) \\
& \Longleftrightarrow \sigma(X) \in \mathfrak{h}\left(i, j, P_{i j}^{(2)}\right) \\
& \Longleftrightarrow \psi_{i j}^{(2)}(\sigma(X))=\sigma(X) \\
& \Longleftrightarrow\left(\sigma^{-1} \circ \psi_{i j}^{(2)} \circ \sigma\right)(X)=X,
\end{aligned}
$$

isto é, $\psi_{i j}^{(2)}=\sigma \circ \psi_{i j}^{(1)} \circ \sigma^{-1}$. Pelas observações acima, podemos concluir que $P_{i j}^{(2)}=$ $\sigma_{j} \circ P_{i j}^{(1)} \circ \sigma_{i}^{-1}$ com $\sigma_{i}$ automorfismo interno de $\mathfrak{g}_{i}$ e $\sigma_{j}$ automorfismo interno de $\mathfrak{g}_{j}$. Provamos então que $\mathfrak{h}\left(i_{1}, j_{1}, P_{i_{1} j_{1}}\right)$ e $\mathfrak{h}\left(i_{2}, j_{2}, P_{i_{2} j_{2}}\right)$ são conjugadas em $\mathfrak{g}$ se e somente se $\psi_{i j}^{(2)}$ e $\psi_{i j}^{(1)}$ são levados um no outro por automorfismos internos de $\mathfrak{g}$, o que é equivalente a $P_{i j}^{(2)}$ ser levado em $P_{i j}^{(1)}$ por automorfismos internos de $\mathfrak{g}_{i}$ e de $\mathfrak{g}_{j}$.

Este teorema juntamente com o teorema 1.2.1 reduz o problema de classificação das subalgebras maximais das álgebras de Lie semisimples a menos de conjugação ao problema de classificação das subalgebras maximais das álgebras de Lie simples a menos de conjugação: este problema será discutido na próxima seção.

Seja $\mathfrak{g}$ uma álgebra de Lie e $\tau$ um automorfismo qualquer de $\mathfrak{g}$. Defina a subalgebra $\mathfrak{g}_{\tau}$ de $\mathfrak{g} \oplus \mathfrak{g}$ pondo

$$
\mathfrak{g}_{\tau}=\{(X, \tau(X)) \mid X \in \mathfrak{g}\}
$$

e extenda o automorfismo $\tau$ de $\mathfrak{g}$ para um automorfismo $\tau^{\prime} \operatorname{de} \mathfrak{g} \oplus \mathfrak{g}$ pondo

$$
\begin{array}{ccc}
\tau^{\prime}: \mathfrak{g} \oplus \mathfrak{g} & \longrightarrow & \mathfrak{g} \oplus \mathfrak{g} \\
X+Y & \longmapsto & \tau^{-1}(Y)+\tau(X)
\end{array}
$$


de modo que $\mathfrak{g}_{\tau}$ é a subalgebra dos pontos fixos de $\tau^{\prime}$. Por exemplo, se $\tau=1$, então $\tau^{\prime}=1$ também, e $\mathfrak{g}_{1}$ é a inclusão diagonal de $\mathfrak{g}$ em $\mathfrak{g} \oplus \mathfrak{g}$. Dizemos que dois automorfismos $\tau_{1}$ e $\tau_{2}$ de uma álgebra de Lie $\mathfrak{g}$ são conjugados se existe um automorfismo interno $\sigma$ de $\mathfrak{g}$ tal que $\tau_{1}=\sigma \tau_{2} \sigma^{-1}$. É claro então que $\mathfrak{g}_{\tau_{1}}$ e $\mathfrak{g}_{\tau_{2}}$ são conjugadas em $\mathfrak{g} \oplus \mathfrak{g}$ se e somente se $\tau_{1}$ e $\tau_{2}$ são conjugados. Como $\operatorname{Int}(\mathfrak{g})$ é um subgrupo normal do grupo Aut $(\mathfrak{g})$, o conjunto das classes de conjugação de automorfismos de uma álgebra de Lie $\mathfrak{g}$ é o grupo quociente Aut $(\mathfrak{g}) / \operatorname{Int}(\mathfrak{g})$, chamado grupo de automorfismos externos de $\mathfrak{g}$. No caso em que a álgebra $\mathfrak{g}$ só possui automorfismos internos, todas as inclusões diagonais de $\mathfrak{g} \mathrm{em} \mathfrak{g} \oplus \mathfrak{g}$ são conjugadas à inclusão canônica dada por $\tau=1$. Por outro lado se $\mathfrak{g}$ possui automorfismos externos o número de classes de conjugação de inclusões será igual ao número de classes de conjugação de automorfismos de $\mathfrak{g}$. Quando a álgebra $\mathfrak{g}$ é semisimples, o grupo $\operatorname{Int}(\mathfrak{g})$ é igual à componente conexa de 1 do grupo $A u t(\mathfrak{g})$ e portanto a ordem do grupo de automorfismos externos é igual ao número de componentes conexas do grupo $\operatorname{Aut}(\mathfrak{g})$. É um fato bem conhecido que quando $\mathfrak{g}$ é semisimples seu grupo de autmorfismos externos $\operatorname{Aut}(\mathfrak{g}) / \operatorname{Int}(\mathfrak{g})$ é isomorfo ao grupo dos automorfismos de seu diagrama de Dynkin (ver [15, pag. 498]), isto é, se $\Pi$ é um conjunto de raizes simples de $\mathfrak{g}$ então Aut(П) é o grupo de permutações de П que preservam o ângulo entre duas raizes simples quaisquer e também preservam o comprimento de todas as raizes simples. Estas observações juntamente com o teorema 1.2.2 fornecem o seguinte

Corolário 1.2.1. Seja $\mathfrak{g}$ uma álgebra de Lie semisimples e $\mathfrak{g}=\mathfrak{g}_{1} \oplus \cdots \oplus \mathfrak{g}_{r}$ sua decomposição em soma direta de ideais simples. Então o número de classes de conjugação de uma subalgebra maximal do tipo diagonal $\mathfrak{h}$, isto é

$$
\mathfrak{h}=\bigoplus_{\substack{k=1 \\ k \neq i, j}}^{r} \mathfrak{g}_{k} \oplus \mathfrak{g}_{i j}
$$

onde $i \neq j$ e $\mathfrak{g}_{i j}=\left\{\left(X_{i}, P_{i j}\left(X_{i}\right)\right) \mid X_{i} \in \mathfrak{g}_{i}\right\}$ para algum isomorfismo $P_{i j}: \mathfrak{g}_{i} \rightarrow \mathfrak{g}_{j}$, é igual a ordem do grupo de automorfismos externos da álgebra de Lie simples $\mathfrak{g}_{i j} \cong \mathfrak{g}_{i} \cong \mathfrak{g}_{j}$.

No próximo capítulo, usaremos os teoremas desta seção para analisar o que acontece quando restringimos uma representação irredutível de uma álgebra de Lie semisimples $\mathfrak{g}$ a uma de suas subalgebras maximais, obtendo assim as regras de ramificação necessárias para implementação do modelo de quebra de simetrias para o código genético.

\section{3 Álgebras de Lie Redutivas e Compactas}

Nesta seção definiremos duas classes de álgebras de Lie que são intimamente relacionadas, e a partir destas relações poderemos reduzir o problema de classificação das subalgebras maximais de uma álgebra de Lie compacta ao problema de classificação das subalgebras maximais redutivas de uma álgebra de Lie semisimples. 
Definição 1.3.1. Uma álgebra de Lie $\mathfrak{g}$ real ou complexa é chamada redutiva se o seu radical $\operatorname{Rad} \mathfrak{g}$ é igual ao seu centro $\mathfrak{z}(\mathfrak{g})$.

Pelo teorema de Levi-Malcev [24, pags. 91,92] sabemos que toda álgebra de Lie $\mathfrak{g}$ admite uma decomposição na soma direta (como espaço vetorial) de seu radical Rad $\mathfrak{g}$ (que é o ideal solúvel máximo de $\mathfrak{g}$ ) e uma subalgebra semisimples maximal $\mathfrak{g}_{s s}$, e esta decomposição é única no sentido de que duas subalgebras maximais semisimples que realizam uma tal decomposição são conjugadas em $\mathfrak{g}$. Agora $\operatorname{Rad} \mathfrak{g}=\mathfrak{z}(\mathfrak{g})$ implica que $\mathfrak{g}_{s s}$ também é um ideal em $\mathfrak{g}$ e que $\mathfrak{g}$ é a soma direta (como álgebra de Lie) de Rad $\mathfrak{g}$ e $\mathfrak{g}_{s s}$. É claro que a álgebra derivada de $\mathfrak{g}$ denotada por $[\mathfrak{g}, \mathfrak{g}]$ é igual a $\mathfrak{g}_{s s}$. Logo, uma álgebra de Lie redutiva se decompõe de forma única na soma direta de uma álgebra abeliana (seu centro) e uma álgebra semisimples (sua subalgebra derivada). Observe que qualquer subespaço do centro de uma álgebra de Lie $\mathfrak{g}$ é um ideal em $\mathfrak{g}$, mostrando que uma álgebra redutiva admite uma decomposição em soma direta (não necessáriamente única) de ideais simples, sendo que alguns destes ideais são abelianos unidimensionais. Portanto, álgebras de Lie abelianas e álgebras de Lie semisimples são exemplos de álgebras de Lie redutivas.

A seguinte proposição dá outra caracterização de álgebras redutivas que será útil para estabelecermos a relação destas com as álgebras compactas.

Proposição 1.3.1 (Knapp [28]). Seja $\mathfrak{g}$ uma álgebra de Lie real ou complexa. São equivalentes:

(i) $\mathfrak{g}$ é redutiva;

(ii) Para todo ideal $\mathfrak{a}$ em $\mathfrak{g}$ existe um ideal $\mathfrak{a}^{\prime}$ complementar a $\mathfrak{a}$ em $\mathfrak{g}$, isto é, tal que $\mathfrak{g}=\mathfrak{a} \oplus \mathfrak{a}^{\prime}$

\section{Demonstração.}

(i) $\Rightarrow$ (ii) Suponhamos que $\mathfrak{g}$ é redutiva, e seja $\mathfrak{a}$ um ideal qualquer em $\mathfrak{g}$; provaremos primeiro que $\mathfrak{a}=\mathfrak{z}(\mathfrak{a}) \oplus[\mathfrak{a}, \mathfrak{a}]$ e além disso que

$$
\mathfrak{z}(\mathfrak{a})=\mathfrak{a} \cap \mathfrak{z}(\mathfrak{g})
$$

e

$$
[\mathfrak{a}, \mathfrak{a}]=\mathfrak{a} \cap[\mathfrak{g}, \mathfrak{g}] .
$$

Claramente $[\mathfrak{a}, \mathfrak{a}] \subset \mathfrak{a} \cap[\mathfrak{g}, \mathfrak{g}]$ e $\mathfrak{a} \cap \mathfrak{z}(\mathfrak{g}) \subset \mathfrak{z}(\mathfrak{a})$. Para mostrar a igualdade, decompomos a álgebra semisimples $[\mathfrak{g}, \mathfrak{g}]=\mathfrak{g}_{s s}$ em soma direta de ideais simples, $[\mathfrak{g}, \mathfrak{g}]=\mathfrak{g}_{1} \oplus \ldots \oplus \mathfrak{g}_{k}$, e usamos o fato de que qualquer ideal de uma álgebra semisimples é soma direta de certos dos seus ideais simples. Por exemplo, $[\mathfrak{a}, \mathfrak{a}]$ e $\mathfrak{a} \cap[\mathfrak{g}, \mathfrak{g}]$ são ideais de $[\mathfrak{g}, \mathfrak{g}] ;$ portanto, $[\mathfrak{a}, \mathfrak{a}] \varsubsetneqq \mathfrak{a} \cap[\mathfrak{g}, \mathfrak{g}]$ implicaria que

$$
[\mathfrak{a}, \mathfrak{a}] \oplus \mathfrak{g}_{1} \oplus \cdots \oplus \mathfrak{g}_{j}=\mathfrak{a} \cap[\mathfrak{g}, \mathfrak{g}]
$$


onde $\mathfrak{g}_{1}, \ldots, \mathfrak{g}_{j}$ seriam certos ideais simples de $[\mathfrak{g}, \mathfrak{g}]$. Mas se $X, Y \in \mathfrak{g}_{\ell}$ para algum $\ell$ $(1 \leqslant \ell \leqslant j)$, então $[X, Y] \in[\mathfrak{a}, \mathfrak{a}] \cap \mathfrak{g}_{\ell}=\{0\}$, isto é, $[X, Y]=0$ para todo $X, Y \in \mathfrak{g}_{\ell}$, o que é absurdo, pois $\mathfrak{g}_{\ell}$ é ideal simples. Logo

$$
[\mathfrak{a}, \mathfrak{a}]=\mathfrak{a} \cap[\mathfrak{g}, \mathfrak{g}] .
$$

De forma análoga, para mostrar que $\mathfrak{z}(\mathfrak{a}) \subset \mathfrak{z}(\mathfrak{g})$, suponha que $A \in \mathfrak{z}(\mathfrak{a})$, e seja $\mathfrak{g}_{i}$ um ideal simples qualquer de $[\mathfrak{g}, \mathfrak{g}]$. Se $\mathfrak{g}_{i} \subset[\mathfrak{a}, \mathfrak{a}]$ então para $X_{i} \in \mathfrak{g}_{i}, X_{i} \in \mathfrak{a}$ e portanto $\left[A, X_{i}\right]=0$. Se $\mathfrak{g}_{i} \not \subset[\mathfrak{a}, \mathfrak{a}], \mathfrak{g}_{i} \cap[\mathfrak{a}, \mathfrak{a}]=\{0\}$ e, para $X_{i} \in \mathfrak{g}_{i},\left[A, X_{i}\right] \in \mathfrak{g}_{i} \cap \mathfrak{a} \cap[\mathfrak{g}, \mathfrak{g}]$ e, pela relação que acabamos de demonstrar, $\left[A, X_{i}\right] \in \mathfrak{g}_{i} \cap[\mathfrak{a}, \mathfrak{a}]=\{0\}$, isto é, $\left[A, X_{i}\right]=0$. Portanto, $[A, X]=0$ para $X \in[\mathfrak{g}, \mathfrak{g}]$, e como é óbvio que $[A, X]=0$ para $X \in \mathfrak{z}(\mathfrak{g})$, concluímos que $[A, X]=0$ para $X \in \mathfrak{g}$, isto é, $A \in \mathfrak{z}(\mathfrak{g})$. Logo

$$
\mathfrak{z}(\mathfrak{a})=\mathfrak{a} \cap \mathfrak{z}(\mathfrak{g}) .
$$

Isso posto, podemos após uma permutação dos índices escrever

$$
[\mathfrak{a}, \mathfrak{a}]=\mathfrak{a} \cap[\mathfrak{g}, \mathfrak{g}]=\mathfrak{g}_{1} \oplus \ldots \oplus \mathfrak{g}_{\ell}
$$

com $\ell \leqslant k$, isto é, temos apenas duas possibilidades: $\mathfrak{g}_{i} \subset[\mathfrak{a}, \mathfrak{a}]$ se $i \leqslant \ell$ e $[\mathfrak{a}, \mathfrak{a}] \cap \mathfrak{g}_{i}=\{0\}$ se $i>\ell$. Agora, seja $A \in \mathfrak{a}$, e escrevemos $A=A_{0}+A_{1}+\ldots+A_{k} \operatorname{com} A_{0} \in \mathfrak{z}(\mathfrak{g})$ e $A_{i} \in \mathfrak{g}_{i}$ $(1 \leqslant i \leqslant k)$. Seja $\mathfrak{g}_{j}$ um ideal simples qualquer de $[\mathfrak{g}, \mathfrak{g}]$. Então para $X_{j} \in \mathfrak{g}_{j}$,

$$
\left[A, X_{j}\right]=\left[A_{1}, X_{j}\right]+\ldots+\left[A_{k}, X_{j}\right]=\left[A_{j}, X_{j}\right] \in \mathfrak{a} \cap \mathfrak{g}_{j}=[\mathfrak{a}, \mathfrak{a}] \cap \mathfrak{g}_{j}
$$

e portanto, $A_{j} \in \mathfrak{z}\left(\mathfrak{g}_{j}\right)=\{0\}$, isto é, $A_{j}=0$ para $j>l$. Logo $A=A_{0}+A_{1}+\cdots+A_{l}$ com $A_{0} \in \mathfrak{z}(\mathfrak{a})$ e $A_{i} \in \mathfrak{g}_{i}(1 \leqslant i \leqslant l)$, o que mostra que

$$
\mathfrak{a}=\mathfrak{z}(\mathfrak{a}) \oplus[\mathfrak{a}, \mathfrak{a}] .
$$

Finalmente, para demonstrar a afirmação (i) $\Rightarrow$ (ii) basta definir $\mathfrak{a}^{\prime}$ como sendo a soma direta de um subespaço complementar a $\mathfrak{z}(\mathfrak{a})$ em $\mathfrak{z}(\mathfrak{g}$ ) (que é um ideal em $\mathfrak{g}$ ) com o ideal complementar a $[\mathfrak{a}, \mathfrak{a}]$ em $[\mathfrak{g}, \mathfrak{g}]: \mathfrak{a}^{\prime}$ é um ideal em $\mathfrak{g}$ que satisfaz

$$
\mathfrak{a} \oplus \mathfrak{a}^{\prime}=\mathfrak{g} .
$$

(ii) $\Rightarrow$ (i) Seja $\mathfrak{a}$ um ideal de $\mathfrak{g}$ e $\mathfrak{a}^{\prime}$ um ideal de $\mathfrak{g}$ complementar a $\mathfrak{a}$. Se $\mathfrak{b}$ é um ideal qualquer de $\mathfrak{a}, \mathfrak{b}$ também é ideal de $\mathfrak{g}$, pois $[\mathfrak{b}, \mathfrak{a}] \subset \mathfrak{b}$ e $\left[\mathfrak{b}, \mathfrak{a}^{\prime}\right] \subset \mathfrak{a} \cap \mathfrak{a}^{\prime}=\{0\}$. Portanto, existe um ideal $\mathfrak{b}^{\prime}$ de $\mathfrak{g}$ complementar a $\mathfrak{b}$ em $\mathfrak{g}$, e $\mathfrak{a} \cap \mathfrak{b}^{\prime}$ é ideal de $\mathfrak{a}$ complementar a $\mathfrak{b}$ em $\mathfrak{a}$. Portanto concluímos que se $\mathfrak{g}$ satisfaz a propriedade (ii) então todo ideal de $\mathfrak{g}$ também satisfaz esta propriedade. Se $\mathfrak{g}$ admite ideais próprios não triviais, podemos escolher um ideal $\mathfrak{g}_{1}$ próprio não-trivial de $\mathfrak{g}$ e um ideal $\mathfrak{g}_{1}^{\prime}$ de $\mathfrak{g}$ complementar a $\mathfrak{g}_{1}$ em $\mathfrak{g}$. Repetindo 
este processo decompomos $\mathfrak{g}$ em uma soma direta de ideais que não contêm ideais próprios não-triviais. Escrevemos esta soma direta na forma

$$
\mathfrak{g}=\mathfrak{a}_{1} \oplus \ldots \oplus \mathfrak{a}_{j} \oplus \mathfrak{a}_{j+1} \oplus \ldots \oplus \mathfrak{a}_{k},
$$

onde $\mathfrak{a}_{1}, \ldots, \mathfrak{a}_{j}$ são unidimensionais e $\mathfrak{a}_{j+1}, \ldots, \mathfrak{a}_{k}$ são álgebras de Lie simples. Como $\left[\mathfrak{a}_{\ell}, \mathfrak{a}_{\ell}\right]=\mathfrak{a}_{\ell}$ para $\ell=j+1, \ldots, k$, temos

$$
[\mathfrak{g}, \mathfrak{g}]=\left[\mathfrak{a}_{j+1}, \mathfrak{a}_{j+1}\right] \oplus \ldots \oplus\left[\mathfrak{a}_{k}, \mathfrak{a}_{k}\right]=\mathfrak{a}_{j+1} \oplus \ldots \oplus \mathfrak{a}_{k},
$$

que é semisimples. Agora provaremos que $\mathfrak{z}(\mathfrak{g})=\mathfrak{a}_{1} \oplus \ldots \oplus \mathfrak{a}_{j}$. Claramente, $\mathfrak{z}(\mathfrak{g}) \supset \mathfrak{a}_{1} \oplus \ldots \oplus \mathfrak{a}_{j}$. Na outra direção, se $X=X_{1}+\ldots+X_{k}$ está em $\mathfrak{z}(\mathfrak{g}) \operatorname{com} X_{i} \in \mathfrak{a}_{i}$, então $X_{i} \in \mathfrak{z}\left(\mathfrak{a}_{i}\right)$ que é $\{0\}$ para $i>j$, pois $\mathfrak{a}_{i}$ é simples nestes casos. Portanto $X=X_{1}+\ldots+X_{j}$, e concluímos que $\mathfrak{z}(\mathfrak{g}) \subset \mathfrak{a}_{1} \oplus \ldots \oplus \mathfrak{a}_{j}$.

Finalmente, para descrever a estrutura do grupo $\operatorname{Aut}(\mathfrak{g})$ de automorfismos e do grupo $\operatorname{Int}(\mathfrak{g})$ de automorfismos internos de uma álgebra redutiva $\mathfrak{g}$, observamos primeiro que uma álgebra de Lie abeliana $\mathfrak{h}$ não possui automorfismos internos não-triviais, porém qualquer transformação linear é um automorfismo (mais precisamente, um automorfismo externo) de $\mathfrak{h}$. Tendo em vista que automorfismos (arbitrários/internos) $\sigma$ de $\mathfrak{g}=\mathfrak{z}(\mathfrak{g}) \oplus \mathfrak{g}_{s s}$ levam $\mathfrak{z}(\mathfrak{g})$ em $\mathfrak{z}(\mathfrak{g})$ e $\mathfrak{g}_{s s}=[\mathfrak{g}, \mathfrak{g}]$ em $\mathfrak{g}_{s s}=[\mathfrak{g}, \mathfrak{g}]$ e portanto se decompõem em soma direta de automorfismos (arbitrários/internos) $\sigma_{\mathfrak{z}}$ de $\mathfrak{z}(\mathfrak{g})$ e $\sigma_{s s}$ de $\mathfrak{g}_{s s}$, temos

$$
\begin{aligned}
\operatorname{Aut}(\mathfrak{g}) & =G L(\mathfrak{z}(\mathfrak{g})) \times \operatorname{Aut}\left(\mathfrak{g}_{s s}\right) \\
\operatorname{Int}(\mathfrak{g}) & =\operatorname{Int}\left(\mathfrak{g}_{s s}\right) .
\end{aligned}
$$

Agora passaremos a discutir uma outra classe de álgebras e Lie, as álgebras de Lie compactas, que têm papel fundamental em toda teoria de álgebras e grupos de Lie.

Definição 1.3.2. Seja $\mathfrak{g}$ uma álgebra de Lie real. Dizemos que $\mathfrak{g}$ é uma álgebra de Lie compacta se existe uma forma bilinear simétrica invariante $(\cdot, \cdot)$ sobre $\mathfrak{g}$ que é positiva definida. A invariância de $(\cdot, \cdot)$ significa que:

$$
([Z, X], Y)+(X,[Z, Y])=0 \quad \text { para todo } X, Y, Z \in \mathfrak{g} .
$$

Nesta definição há um certo abuso de linguagem, pois uma álgebra de Lie nunca é compacta no sentido topológico, pois é um espaço vetorial real; por isso alguns autores usam o termo "álgebra de tipo compacto".

A identidade (1.4) é a versão "infinitesimal" da afirmação de que o produto escalar $(\cdot, \cdot)$ é invariante sob o grupo de automorfismos internos de $\mathfrak{g}$, isto é

$$
(\sigma(X), \sigma(Y))=(X, Y)
$$


para todo $X, Y \in \mathfrak{g}$ e todo $\sigma \in \operatorname{Int}(\mathfrak{g})$. Observe que (1.5) implica que, relativamente a este produto escalar, o grupo $\operatorname{Int}(\mathfrak{g})$ age por transformações ortogonais e portanto a aplicação $\operatorname{ad}(Z)$ é anti-simétrica para todo $Z \in \mathfrak{g}$.

Obviamente, toda subalgebra de uma álgebra de Lie compacta é também uma álgebra de Lie compacta. Uma outra consequência óbvia da definição 1.3.2, é que toda álgebra de Lie real abeliana é compacta, pois, neste caso, a condição de invariância (1.4 é trivialmente satisfeita por qualquer forma bilinear, e em particular pelo produto escalar canônico de um espaço vetorial real.

A principal motivação para a definição 1.3.2 é sugerida pela seguinte proposição.

Proposição 1.3.2. Seja $G$ um grupo de Lie compacto e $\mathfrak{g}$ sua álgebra de Lie. Então $\mathfrak{g}$ é uma álgebra de Lie compacta.

DemonstraÇÃo. Usando integração sobre o grupo $\operatorname{Ad}(G)$, que também é compacto, introduzimos um produto escalar $(\cdot, \cdot)$ invariante, começando por um produto escalar qualquer $\langle\cdot, \cdot\rangle$, pondo:

$$
(X, Y)=\int_{\operatorname{Ad}(G)}\langle g X, g Y\rangle d \mu(g),
$$

onde $\mu$ é a medida de Haar bi-invariante em $\operatorname{Ad}(G)$ normalizada por $\mu(\operatorname{Ad}(G))=1$ (para a construção de $\mu$ veja Warner [56, pag. 151] ou Bröcker \& tomDieck [7, pag. 40]). É fácil ver que $(\cdot, \cdot)$ é positiva definida, pois para todo $X \in \mathfrak{g}$, a função $\langle\cdot X, \cdot X\rangle$ sobre $\operatorname{Ad}(G)$ é não-negativa e se anula se e somente se $X=0$. Só falta mostrar que $(\cdot, \cdot)$ é invariante. De fato, se $h=\operatorname{Ad}(k) \in \operatorname{Ad}(G)$ é fixo, temos

$$
\begin{aligned}
(\operatorname{Ad}(k) X, \operatorname{Ad}(k) Y) & =\int_{\operatorname{Ad}(G)}\langle g h X, g h Y\rangle d \mu(g) \\
& =\int_{\operatorname{Ad}(G)}\langle l X, l Y\rangle d \mu\left(l h^{-1}\right) \\
& =\int_{\operatorname{Ad}(G)}\langle l X, l Y\rangle d \mu(l) \\
& =(X, Y)
\end{aligned}
$$

e portanto para todo $k \in G$ a transformação $\operatorname{Ad}(k)$ é ortogonal. Isto é equivalente a

$$
(\operatorname{ad}(Z) X, Y)+(X, \operatorname{ad}(Z) Y)=0
$$

para todo $X, Y, Z \in \mathfrak{g}$, demonstrando assim que $(\cdot, \cdot)$ satisfaz a identidade (1.4).

Teorema 1.3.1. Seja $\mathfrak{g}$ uma álgebra de Lie compacta. Então $\mathfrak{g}$ é redutiva, e portanto $\mathfrak{g}=$ $\mathfrak{z}(\mathfrak{g}) \oplus[\mathfrak{g}, \mathfrak{g}]$, onde $\mathfrak{z}(\mathfrak{g})$ é o centro de $\mathfrak{g}$ e $[\mathfrak{g}, \mathfrak{g}]$ é semisimples. 
DemonstraÇÃo. Seja $(\cdot, \cdot)$ uma forma bilinear simétrica invariante e positiva definida em $\mathfrak{g}$. Os subespaços invariantes de $\mathfrak{g} \operatorname{sob}$ ad($(\mathfrak{g})$ são os ideais de $\mathfrak{g}$. Portanto, se $\mathfrak{a}$ é um ideal de $\mathfrak{g}$, então seu complemento ortogonal

$$
\mathfrak{a}^{\perp}=\{X \in \mathfrak{g} \mid(X, Y)=0 \quad \text { para todo } Y \in \mathfrak{a}\}
$$

também é um ideal de $\mathfrak{g}$. Como $(\cdot, \cdot)$ é positiva definida, $\mathfrak{g}=\mathfrak{a} \oplus \mathfrak{a}^{\perp}$. Portanto $\mathfrak{a}$ tem $\mathfrak{a}^{\perp}$ como ideal complementar e, pela proposição 1.3.1, g é redutiva.

O próximo teorema é o clássico "critério de Weyl”, que caracteriza as álgebras de Lie semisimples que são compactas.

Teorema 1.3.2 (Weyl). Seja $\mathfrak{g}$ uma álgebra de Lie real semisimples. Então $\mathfrak{g}$ é compacta se e somente se a sua forma de Killing é negativa definida.

DemonstraçÃo. Se a forma de Killing de $\mathfrak{g}$ é negativa definida, então é óbvio que $\mathfrak{g}$ é compacta. Reciprocamente, suponhamos que $\mathfrak{g}$ é compacta e que $(\cdot, \cdot)$ seja uma forma invariante positiva definida sobre $\mathfrak{g}$. A condição de invariância implica que para todo $X \in \mathfrak{g}$, $\operatorname{ad}(X)$ é anti-simétrico: isto significa que $\operatorname{ad}(X)$ é diagonalizável sobre $\mathbb{C}$ e que todos os seus valores próprios são imaginários puros. Portanto, $\operatorname{tr}\left(\operatorname{ad}(X)^{2}\right) \leqslant 0$ e, como g é semisimples, $\operatorname{tr}\left(\operatorname{ad}(X)^{2}\right)=0$ se e somente se $X=0$.

Corolário 1.3.1. Toda álgebra de Lie compacta é isomorfa à álgebra de Lie de um grupo de Lie compacto.

DemonstraÇÃo. Seja $\mathfrak{k}$ uma álgebra de Lie compacta, então como $\mathfrak{k}$ é redutiva podemos escrever $\mathfrak{k}=\mathfrak{z}(\mathfrak{k}) \oplus[\mathfrak{k}, \mathfrak{k}]$, e basta mostrar que a cada um destes dois ideais corresponde um grupo de Lie compacto, pois então o produto direto destes dois grupos tem álgebra de Lie isomorfa a $\mathfrak{k}$ (ver Knapp [28, pag. 58]) e é obviamente compacto. Como $\mathfrak{z}(\mathfrak{k})$ é abeliana, ela é isomorfa à álgebra de Lie de um torus, que é um grupo de Lie compacto. Falta mostrar que $\mathfrak{l}=[\mathfrak{k}, \mathfrak{k}]$ é isomorfa à álgebra de Lie de um grupo compacto. Como $\mathfrak{l}$ é semisimples, ela é isomorfa a $\operatorname{ad}(\mathfrak{l})$ que é a álgebra de Lie do grupo de Lie $\operatorname{Int}(\mathfrak{l})$ que por sua vez é a componente conexa de 1 do grupo $\operatorname{Aut}(\mathfrak{l}) \subset G L(\mathfrak{l})$ e portanto $\operatorname{Int}(\mathfrak{l})$ é fechado em $G L(\mathfrak{l})$. Por outro lado, cada membro de ad(l) é uma transformação anti-simétrica e portanto o grupo correspondente $\operatorname{Int}(\mathfrak{l})$ age por transformações ortogonais. Exibimos então um grupo de Lie que possui álgebra de Lie isomorfa a $\mathfrak{l}$ e, sendo um subgrupo fechado do grupo $S O(\mathfrak{l})$, é compacto.

Juntos, a proposição 1.3.2 e o corolário 1.3.1 justificam o abuso de linguagem do termo "álgebra de Lie compacta".

$\mathrm{Na}$ seção anterior mostramos como determinar as subalgebras maximais de uma álgebra de Lie semisimples. Agora mostraremos como determinar as subalgebras maximais de uma álgebra de Lie redutiva. 
Teorema 1.3.3. Seja $\mathfrak{g}$ uma álgebra de Lie redutiva e $\mathfrak{g}=\mathfrak{z}(\mathfrak{g}) \oplus[\mathfrak{g}, \mathfrak{g}]$ sua decomposição na soma direta de seu centro e sua álgebra derivada. As subalgebras maximais $\mathfrak{h}$ de $\mathfrak{g}$ são da forma

$$
\mathfrak{h}=\mathfrak{z}(\mathfrak{g}) \oplus \mathfrak{f}
$$

onde $\mathfrak{f}$ é uma subalgebra maximal de $[\mathfrak{g}, \mathfrak{g}]$.

Demonstração. Seja $\mathfrak{f}$ uma subalgebra maximal de $[\mathfrak{g}, \mathfrak{g}]$, então a subalgebra $\mathfrak{h}=\mathfrak{z}(\mathfrak{g}) \oplus$ $\mathfrak{f}$ é obviamente maximal em $\mathfrak{g}$. Reciprocamente, seja $\mathfrak{h}$ uma subalgebra maximal de $\mathfrak{g}$. Obviamente temos que

$$
\mathfrak{h} \supset(\mathfrak{z}(\mathfrak{g}) \cap \mathfrak{h}) \oplus([\mathfrak{g}, \mathfrak{g}] \cap \mathfrak{h}) .
$$

Porém $\mathfrak{h}$ não pode conter $[\mathfrak{g}, \mathfrak{g}]$, pois senão teríamos que $\mathfrak{h}=(\mathfrak{z}(\mathfrak{g}) \cap \mathfrak{h}) \oplus[\mathfrak{g}, \mathfrak{g}]$ e a única possibilidade de ter $\mathfrak{h}$ maximal seria $\operatorname{com} \mathfrak{z}(\mathfrak{g}) \cap \mathfrak{h}=\mathfrak{z}(\mathfrak{g})$, o que contraria a hipótese de que $\mathfrak{h} \neq \mathfrak{g}$. Por outro lado $\mathfrak{h}$ deve conter $\mathfrak{z}(\mathfrak{g})$. De fato, se existisse $X \in \mathfrak{z}(\mathfrak{g})$ tal que $X \notin \mathfrak{h}$ a subalgebra $\mathfrak{h}^{\prime}$ gerada por $X$ e $\mathfrak{h}$ seria igual a $\mathfrak{g}$, portanto $[\mathfrak{g}, \mathfrak{g}] \subset \mathfrak{h}$, e como já mostramos isto não pode ocorrer. Portanto, definindo a subalgebra $\mathfrak{f}$ de $[\mathfrak{g}, \mathfrak{g}]$ por

$$
\mathfrak{f}=[\mathfrak{g}, \mathfrak{g}] \cap \mathfrak{h},
$$

temos que $\mathfrak{h}=\mathfrak{z}(\mathfrak{g}) \oplus \mathfrak{f}$ com $\mathfrak{f}$ subalgebra maximal de $[\mathfrak{g}, \mathfrak{g}]$.

Portanto, para determinar todas as subalgebras redutivas maximais de uma álgebra de Lie redutiva $\mathfrak{g}$, basta determinar todas as subalgebras redutivas maximais de $[\mathfrak{g}, \mathfrak{g}]$. Mostraremos agora que a classificação das subalgebras maximais de uma álgebra de Lie semisimples compacta é equivalente à classificação das subalgebras redutivas maximais da sua complexificação. Para tanto precisamos discutir a relação entre álgebras de Lie compactas e álgebras de Lie complexas redutivas, e suas respectivas subalgebras. Obviamente a complexificação de uma álgebra de Lie compacta $\mathfrak{k}$ fornece uma álgebra de Lie complexa redutiva $\mathfrak{g}=\mathfrak{k}^{\mathbb{C}}$, e como toda subalgebra $\mathfrak{l}$ de $\mathfrak{k}$ é compacta (e portanto redutiva), sua complexificação $\mathfrak{f}=\mathfrak{l}^{\mathbb{C}}$ será uma subalgebra complexa redutiva de $\mathfrak{g}$. Mas em geral nem toda subalgebra complexa redutiva $\mathfrak{f}$ de $\mathfrak{g}=\mathfrak{k}^{\mathbb{C}}$ corresponde a uma subalgebra $\mathfrak{l}$ de $\mathfrak{k}$ tal que $\mathfrak{f}=\mathfrak{l}^{\mathbb{C}}$.

Para analisar a questão quais subalgebras complexas redutivas de $\mathfrak{g}=\mathfrak{k}^{\mathbb{C}}$ provêm de subalgebras de $\mathfrak{k}$ por complexificação, observemos primeiro que devido ao teorema 1.3.3 acima, podemos nos restringir a responder esta pergunta para o caso em que $\mathfrak{k}$ e $\mathfrak{g}$ são semisimples.

No caso em que $\mathfrak{f}$ é subalgebra complexa semisimples de $\mathfrak{g}$, podemos afirmar que $\mathfrak{f}=\mathfrak{l}^{\mathbb{C}}$ para alguma subalgebra $\mathfrak{l}$ de $\mathfrak{k}$. De fato, $\mathfrak{f}$ possui uma forma real compacta $\mathfrak{l}$ que está contida em uma subalgebra compacta maximal $\mathfrak{k}$ de $\mathfrak{g}$. Mas todas as subalgebras compactas maximais de uma álgebra de Lie complexa semisimples são conjugadas ([19, pag. 256, teo. 2.2]) e toda forma real compacta de uma álgebra de Lie complexa semisimples é uma 
subalgebra compacta maximal ([19, pag. 184, prop. 7.3]). Portanto, k é uma forma real compacta de $\mathfrak{g}$ e $\mathfrak{l} \subset \mathfrak{f} \cap \mathfrak{k}$, mas $\mathfrak{l}$ é subalgebra compacta maximal de $\mathfrak{f}, \log 0 \mathfrak{l}=\mathfrak{f} \cap \mathfrak{k}$.

Para analisar o caso em que $\mathfrak{f}$ é uma subalgebra redutiva de $\mathfrak{g}$ consideremos a decomposição de Cartan de $\mathfrak{g}$,

$$
\mathfrak{g}=\mathfrak{k} \oplus i \mathfrak{k}
$$

junto com a correspondente involução de Cartan $\theta$, que é o automorfismo de $\mathfrak{g}$ (como álgebra de Lie real) definido como a identidade sobre $\mathfrak{k}$ e menos a identidade sobre $i \mathfrak{k}$. Observamos que uma subalgebra $\mathfrak{f}$ de $\mathfrak{g}$ é a complexificação de uma subalgebra $\mathfrak{l}$ de $\mathfrak{k}$ se e somente se $\mathfrak{f}$ é $\theta$-invariante:

$$
\theta(\mathfrak{f})=\mathfrak{f} .
$$

Portanto, podemos reformular a afirmação anterior como dizendo que qualquer subalgebra complexa semisimples $\mathfrak{f}$ de $\mathfrak{g}$ é necessariamente $\theta$-invariante. Além disto, pode-se provar que toda subalgebra $\theta$-invariante de $\mathfrak{g}$ é necessariamente redutiva ([28, pag. 304, cor. 6.29]).

Definição 1.3.3. Uma subalgebra $\mathfrak{f}$ de uma álgebra de Lie complexa redutiva $\mathfrak{g}$ é dita de posto máximo se $\mathfrak{f}$ contém alguma subalgebra de Cartan $\mathfrak{h}$ de $\mathfrak{g}$.

Uma subalgebra de Cartan $\mathfrak{h}$ de uma álgebra de Lie complexa redutiva $\mathfrak{g}$ (como no caso das álgebras semisimples) é uma subalgebra abeliana maximal tal que ad $(X)$ é semisimples para todo $X \in \mathfrak{h}$. É fácil ver que $\mathfrak{h}$ é a soma direta de uma subalgebra de Cartan da subalgebra derivada $[\mathfrak{g}, \mathfrak{g}] \operatorname{com} \mathfrak{z}(\mathfrak{g})$. É claro então que o centro de $\mathfrak{g}$ é a intersecção de todas as subalgebras de Cartan de $\mathfrak{g}$. Ademais se $\mathfrak{f}$ é uma subalgebra redutiva de posto máximo de uma álgebra de Lie semisimples $\mathfrak{g}$, então uma subalgebra de Cartan de $\mathfrak{f}$ também é uma subalgebra de Cartan de $\mathfrak{g}$. Lembremos também que, fixada uma decomposição de Cartan de uma álgebra de Lie complexa semisimples $\mathfrak{g}$ com involução $\theta$, sempre existe uma subalgebra de Cartan de $\mathfrak{g}$ que é $\theta$-invariante ([55, pag. 57]).

Teorema 1.3.4. Seja $\mathfrak{g}$ uma álgebra de Lie complexa semisimples com forma real compacta $\mathfrak{k}$ e seja e $\mathfrak{f}$ uma subalgebra redutiva maximal de $\mathfrak{g}$ com centro não-trivial. Se $\mathfrak{f}$ é a complexificação de uma subalgebra $\mathfrak{l}$ de $\mathfrak{k}$ então $\mathfrak{f}$ é uma subalgebra de posto máximo. Reciprocamente, se $\mathfrak{f}$ é uma subalgebra de posto máximo então $\mathfrak{f}$ é conjugada a uma subalgebra de $\mathfrak{g}$ que é complexificação de uma subalgebra de $\mathfrak{k}$.

DemonstraÇÃo. Suponhamos primeiro que existe uma subalgebra $\mathfrak{l}$ de $\mathfrak{k}$ tal que $\mathfrak{f}=\mathfrak{l}^{\mathbb{C}}$. Como f é subalgebra redutiva maximal de $\mathfrak{g}$ com centro não-trivial, $\mathfrak{l}$ é subalgebra maximal de $\mathfrak{k}$ com centro não-trivial (De fato, se o centro de $\mathfrak{l}$ fosse trivial, $\mathfrak{l}$ seria semisimples e portanto $\mathfrak{f}$ seria semisimples). Seja $X$ um elemento não-nulo qualquer do centro de $\mathfrak{l}$, e seja $\mathfrak{z} \mathfrak{k}(X)$ seu centralizador em $\mathfrak{k}$. Mas obviamente, $\mathfrak{l} \subset \mathfrak{z}_{\mathfrak{k}}(X)$ (pois $X$ está no centro de $\mathfrak{l}$ ), e como $\mathfrak{l}$ é maximal em $\mathfrak{k}$, concluímos que $\mathfrak{z}_{\mathfrak{k}}(X)=\mathfrak{l}$ ou $\mathfrak{z}_{\mathfrak{k}}(X)=\mathfrak{k}$. O segundo caso é excluído porque 
$\mathfrak{g}$ e $\mathfrak{k}$ são semisimples. Seja agora $\mathfrak{t}$ uma subalgebra abeliana maximal de $\mathfrak{k}$ que contém $X$ : então

$$
\mathfrak{t}=\mathfrak{z}_{\mathfrak{k}}(\mathfrak{t}) \subset \mathfrak{z}_{\mathfrak{k}}(X)=\mathfrak{l},
$$

e portanto $\mathfrak{h}=\mathfrak{t}^{\mathbb{C}}$ é uma subalgebra de Cartan de $\mathfrak{g}$ contida em $\mathfrak{f}$.

Reciprocamente, suponhamos que $\mathfrak{f}$ é uma subalgebra redutiva de $\mathfrak{g}$ com centro não-trivial e de posto máximo. Escrevemos $\mathfrak{f}=\mathfrak{z}(\mathfrak{f}) \oplus[\mathfrak{f}, \mathfrak{f}]$ e observamos que existe uma subalgebra de Cartan $\mathfrak{h}$ de $\mathfrak{g}$ contida em $\mathfrak{f}$; portanto temos $\mathfrak{z}(\mathfrak{f}) \subset \mathfrak{h} \subset \mathfrak{f} .{ }^{1}$ Ademais, como já vimos anteriormente, a parte semisimples $[\mathfrak{f}, \mathfrak{f}]$ de $\mathfrak{f}$ é $\theta$-invariante: $\theta([\mathfrak{f}, \mathfrak{f}])=[\mathfrak{f}, \mathfrak{f}]$. Portanto, precisamos apenas mostrar que o centro $\mathfrak{z}(\mathfrak{f})$ de $\mathfrak{f}$ também é $\theta$-invariante. Seja então $X \in \mathfrak{z}(\mathfrak{f})$. Logo $X \in \mathfrak{h}$ e $\theta(X) \in \mathfrak{h}$ e $\theta(X) \in \mathfrak{f}$. Por outro lado, $\theta(X)$ comuta não apenas com $\mathfrak{z}(\mathfrak{f})$ mas também com $[\mathfrak{f}, \mathfrak{f}]=\theta([\mathfrak{f}, \mathfrak{f}])$ :

$$
[\theta(X), \theta([Y, Z])]=\theta([X,[Y, Z]])=0 \quad \text { para } \quad Y, Z \in \mathfrak{f} .
$$

$\log \theta(X) \in \mathfrak{f}$.

Como veremos na próxima seção, uma subalgebra redutiva maximal de uma álgebra de Lie simples $\mathfrak{g}$ ou é semisimples ou é redutiva de posto máximo com centro unidimensional; portanto, cada classe de conjugação de subalgebras complexas redutivas maximais de $\mathfrak{g}$ define uma classe de conjugação de subalgebras maximais da forma real compacta de $\mathfrak{g}$.

\subsection{Classificação das Subalgebras Maximais das Álgebras de Lie Simples}

Nesta seção enunciamos os teoremas de Dynkin que classificam todas as subalgebras maximais das álgebras de Lie simples: as quatro séries infinitas de álgebras clássicas e as cinco álgebras excepcionais, segundo a classificação de Cartan. Todas as álgebras de Lie nesta seção são complexas, a menos que se mencione explicitamente o contrário.

Como estamos interessados na classificação das subalgebras maximais das álgebras de Lie compactas e os teoremas são formulados para álgebras complexas, vamos considerar sempre subalgebras redutivas. Portanto quando apresentamos uma lista de subalgebras maximais de uma álgebra de Lie $\mathfrak{g}$, fica subentendido que esta lista se refere a subalgebras redutivas maximais, ou seja, subalgebras que são maximais dentro de todas as subalgebras redutivas de $\mathfrak{g}$. É perfeitamente possível que tais subalgebras não sejam maximais dentro

\footnotetext{
${ }^{1}$ Como todas as subalgebras de Cartan de $\mathfrak{g}$ são conjugadas e como a complexificação de qualquer subalgebra abeliana maximal de $\mathfrak{k}$ é uma subalgebra de Cartan de $\mathfrak{g}$, podemos supor sem perda de generalidade (isto é, após efetuarmos uma conjugaçõa apropriada de $\mathfrak{h}$ e $\mathfrak{f}$ ) que $\mathfrak{h}$ é $\theta$-invariante.
} 
de todas as subalgebras de $\mathfrak{g}$ e que existam outras subalgebras maximais, com radical nãoabeliano e portanto não redutivas. Porém, esta ambiguidade é eliminada quando passamos à forma compacta $\mathfrak{k}$ de $\mathfrak{g}$, pois as subalgebras maximais de $\mathfrak{g}$ podem ser agrupadas em subalgebras maximais que contêm uma determinada subalgebra redutiva maximal. De fato, se $\tilde{\mathfrak{f}}$ é uma subalgebra maximal de $\mathfrak{g}$, redutiva ou não, então $\mathfrak{l}=\tilde{\mathfrak{f}} \cap \mathfrak{k}$ é uma subalgebra maximal de $\mathfrak{k}$ e $\mathfrak{f}=\mathfrak{l}^{\mathbb{C}}$ é uma subalgebra redutiva maximal de $\mathfrak{g} \operatorname{com} \mathfrak{f} \subset \tilde{\mathfrak{f}}$.

Nos limitaremos a enunciar e comentar os teoremas, pois a demonstração nos levaria muito longe e não é essencial para a compreensão do modelo algébrico para a evolução do código genético que motivou o presente trabalho. Por outro lado os resultados destes teoremas são fundamentais para a implementação do modelo.

Os resultados que enunciaremos se encontram em dois artigos de Dynkin [10, 11] que por sua vez se baseiam principalmente sobre um trabalho anterior de Malcev [34]. Alguns destes resultados já haviam sido obtidos por Borel e Siebenthal [4] por métodos diferentes, e depois por Golubitsky [16], que também reproduz vários resultados de Morozov e Karpelevich. A forma em que apresentamos os teoremas, que difere um pouco da forma original, se baseia em um artigo de Tits [53] e na apresentação moderna de Onishchik \& Vinberg [42].

Estudaremos primeiro as álgebras clássicas que são naturalmente definidas como álgebras de matrizes e portanto possuem uma representação linear preferencial. Esta representação pode ser caracterizada como sendo a representação irredutível de menor dimensão possível, com algumas exceções: a série $A_{r} \operatorname{com} r \geqslant 2$ e a álgebra $D_{4}$. As álgebras $A_{r}$ possuem duas representações irredutíveis de dimensão mínima que são inequivalentes, sendo que uma é a conjugada complexa da outra: são equivalentes apenas quando $r=1$. A álgebra $D_{4}$ possui três representações irredutíveis de dimensão mínima, 8 no caso, que são inequivalentes: uma vetorial e as outras duas spinoriais. Mas, mesmo nestes casos, podemos fixar uma representação preferencial definindo-a explicitamente. Ademais, quando escolhemos uma representação de uma álgebra complexa $\mathfrak{g}$, fixamos automáticamente uma única representação da sua forma real compacta $\mathfrak{k}$.

Então quando dissermos que uma álgebra de Lie complexa $\mathfrak{g}$ é uma álgebra de Lie clássica complexa, estaremos nos referindo a uma das seguintes álgebras de Lie:

$$
\begin{aligned}
& \mathfrak{s l}(n, \mathbb{C}) \quad=\{X \in \mathfrak{g l}(n, \mathbb{C}) \mid \operatorname{tr}(X)=0\}, \quad \text { para } n \geqslant 2, \\
& \mathfrak{s o}(n, \mathbb{C})=\left\{X \in \mathfrak{g l}(n, \mathbb{C}) \mid X+X^{T}=0\right\}, \quad \text { para } n \geqslant 2, \\
& \mathfrak{s p}(2 n, \mathbb{C})=\left\{X \in \mathfrak{g l}(2 n, \mathbb{C}) \mid X^{T} J_{2 n}+J_{2 n} X=0\right\}, \quad \text { para } n \geqslant 1,
\end{aligned}
$$

onde $X^{T}$ é a matriz transposta de $X$ e $J_{2 n}$ é a matriz $2 n$ por $2 n$ dada por

$$
J_{2 n}=\left(\begin{array}{cc}
0 & 1_{n} \\
-1_{n} & 0
\end{array}\right) \text {. }
$$


É claro que estas definições carregam consigo uma representação linear, que tem dimensão mínima: são estas representações que fixaremos para as álgebras de Lie clássicas. Há formas alternativas de definir as álgebras $\mathfrak{s o}(2 n, \mathbb{C})$ e $\mathfrak{s p}(2 n, \mathbb{C})$ que são muito úteis, e cujas representações lineares associadas são equivalentes às que apresentamos:

$$
\begin{aligned}
& \left.\mathfrak{s o}(2 n, \mathbb{C})=\left\{\begin{array}{cc}
A & B \\
C & -A^{T}
\end{array}\right) \in \mathfrak{g l}(2 n, \mathbb{C}) \mid \begin{array}{c}
A, B, C \in \mathfrak{g} \mathfrak{l}(n, \mathbb{C}) \\
B, C \text { anti-simétricas }
\end{array}\right\}, \\
& \left.\mathfrak{s p}(2 n, \mathbb{C})=\left\{\begin{array}{cc}
A & B \\
C & -A^{T}
\end{array}\right) \in \mathfrak{g l}(2 n, \mathbb{C}) \mid \begin{array}{c}
A, B, C \in \mathfrak{g l}(n, \mathbb{C}) \\
B, C \text { simétricas }
\end{array}\right\} .
\end{aligned}
$$

Uma das vantagens destas definições é que fica óbvia a relação

$$
\mathfrak{s o}(2 n, \mathbb{C}) \cap \mathfrak{s p}(2 n, \mathbb{C})=\mathfrak{g l}(n, \mathbb{C}) .
$$

As respectivas formas compactas das álgebras clássicas são

\begin{tabular}{|c|c|c|c|c|}
\hline $\begin{array}{l}\text { Notação } \\
\text { de Cartan }\end{array}$ & $\begin{array}{c}\text { Álgebra } \\
\text { Complexa }\end{array}$ & $\begin{array}{c}\text { Forma Real } \\
\text { Compacta }\end{array}$ & Dimensão & $\begin{array}{l}\text { Diagrama } \\
\text { de Dynkin }\end{array}$ \\
\hline$A_{r}(r \geqslant 1)$ & $\mathfrak{s l}(r+1, \mathbb{C})$ & $\mathfrak{s u}(r+1)$ & $r(r+2)$ & $\mathrm{O}$ \\
\hline$B_{r}(r \geqslant 2)$ & $\mathfrak{s o}(2 r+1, \mathbb{C})$ & $\mathfrak{s o}(2 r+1)$ & $r(2 r+1)$ & $0-0-\ldots-0-0 \geqslant 0$ \\
\hline$C_{r}(r \geqslant 3)$ & $\mathfrak{s p}(2 r, \mathbb{C})$ & $\mathfrak{s p}(2 r)$ & $r(2 r+1)$ & $\mathrm{O}-\mathrm{O}-\ldots-\mathrm{O}-\mathrm{C}$ \\
\hline$D_{r}(r \geqslant 4)$ & $\mathfrak{s o}(2 r, \mathbb{C})$ & $\mathfrak{s o}(2 r)$ & $r(2 r-1)$ & $O-O-\ldots-\infty-\infty$ \\
\hline
\end{tabular}

$$
\begin{array}{lll}
\mathfrak{s u}(n) & =\left\{X \in \mathfrak{g l}(n, \mathbb{C}) \mid X+X^{\dagger}=0, \operatorname{tr}(X)=0\right\}, & \text { para } n \geqslant 2, \\
\mathfrak{s o}(n) & =\left\{X \in \mathfrak{g l}(n, \mathbb{R}) \mid X+X^{\dagger}=0\right\}, & \\
\mathfrak{s p}(2 n)=\left\{X \in \mathfrak{g l}(n, \mathbb{H}) \mid X+X^{\dagger}=0\right\}, & & \text { para } n \geqslant 2,
\end{array}
$$

onde $X^{\dagger}$ é a matriz transposta conjugada a $X$.

Tabela 1.2: Álgebras de Lie clássicas. 
Assim fixamos para cada álgebra de Lie clássica $\mathfrak{g}$ uma representação especial com dimensão mínima, que chamaremos a representação fundamental de $\mathfrak{g}$.

A tabela 1.2 apresenta as álgebras de Lie clássicas complexas, de acordo com a classificação de Cartan, e suas respectivas formas reais compactas, nas respectivas representações fundamentais, bem como a dimensão e o diagrama de Dynkin de cada uma.

Na tabela 1.3 apresentamos as representações de dimensão mínima das álgebras clássicas na notação que introduzimos na seção 2.1, com seus pesos máximos e suas dimensões. A representação fundamental aparece em negrito.

\begin{tabular}{|c|c|c|}
\hline $\begin{array}{c}\text { Notação } \\
\text { de Cartan }\end{array}$ & $\begin{array}{c}\text { Peso } \\
\text { Máximo }\end{array}$ & $\begin{array}{c}\text { Dimensão da } \\
\text { Representação }\end{array}$ \\
\hline \hline$A_{1}$ & $(\mathbf{1})$ & 2 \\
\hline$A_{r}(r \geqslant 2)$ & $(\mathbf{1 0 \ldots 0}) \mathrm{e}(0 \ldots 01)$ & $r+1$ \\
\hline$B_{r}(r \geqslant 2)$ & $(\mathbf{1 0} \ldots \mathbf{0})$ & $2 r+1$ \\
\hline$C_{r}(r \geqslant 3)$ & $(\mathbf{1 0} \ldots \mathbf{0})$ & $2 r$ \\
\hline$D_{4}$ & $(\mathbf{1 0 0 0}),(0010) \mathrm{e}(0001)$ & 8 \\
\hline$D_{r}(r \geqslant 5)$ & $(\mathbf{1 0} \ldots \mathbf{0})$ & $2 r$ \\
\hline
\end{tabular}

Tabela 1.3: Representações de dimensão mínima das álgebras clássicas.

Observe que as álgebras clássicas que possuem mais de uma representação irredutível de dimensão mínima são exatamente as que possuem automorfismos do diagrama de Dynkin, o que não é nenhuma surpresa: são estes automorfismos que induzem automorfismos externos das respectivas álgebras, e estes por sua vez transformam uma representação em outra, inequivalente. Vale também observar que, devido ao isomorfismo $\mathfrak{s o}(5, \mathbb{C}) \cong \mathfrak{s p}(4, \mathbb{C})$, a álgebra $\mathfrak{s o}(5, \mathbb{C}$ ) possui uma representação de dimensão 4 (a representação fundamental de $\mathfrak{s p}(4, \mathbb{C}))$, mas de acordo com a nossa definição sua representação fundamental tem dimensão 5. Isto não causa problema pois, como veremos no próximo capítulo, os teoremas de classificação fornecem o mesmo resultado independentemente da representação que escolhemos: basta identificar quais subalgebras maximais de $\mathfrak{s o}(5, \mathbb{C})$ correspondem por este isomorfismo as subalgebras maximais de $\mathfrak{s p}(4, \mathbb{C})$. 
Notemos também que, na tabela 1.3, omitimos as vírgulas ao escrevermos os pesos, o que não causa confusão pois todos os números envolvidos estão na faixa $0 \leqslant n \leqslant 9$. Portanto, sabemos que cada algarismo corresponde a um dos coeficientes $p_{i}$ que introduzimos anteriormente, e a notação fica mais fácil de se ler. Neste caso usaremos a vírgula para separar os pesos correspondentes aos ideais simples de uma álgebra semisimples: por exemplo, se $\mathfrak{g}=\mathfrak{s u}(3, \mathbb{C}) \oplus \mathfrak{s o}(8, \mathbb{C})$, escreveremos $(100,1000)$ para indicar a representação de $\mathfrak{g}$ composta pelas representações fundamentais de seus ideais simples.

Para podermos formular os teoremas de classificação, precisaremos de mais algumas definições. Se $\mathfrak{g}$ é uma álgebra de Lie clássica com sua representação fundamental fixada, diremos que uma subalgebra maximal $\mathfrak{f} \subset \mathfrak{g}$ é irredutivel se a restrição da representação fundamental de $\mathfrak{g}$ à subalgebra $\mathfrak{f}$ for irredutível; caso contrário, diremos que $\mathfrak{f}$ é redutivel. Assim dividimos o conjunto das subalgebras maximais ${ }^{1}$ de $\mathfrak{g}$ em três subconjuntos, obviamente disjuntos:

- Subalgebras maximais redutíveis,

- Subalgebras maximais irredutíveis não simples,

- Subalgebras maximais irredutíveis simples.

Lembremos primeiramente dos seguintes isomorfismos entre algumas álgebras de Lie:

$$
\begin{aligned}
& \mathfrak{s l}(1, \mathbb{C})=\mathfrak{s o}(1, \mathbb{C})=\{0\} \\
& \mathfrak{s o}(2, \mathbb{C})=\mathbb{C} \\
& \mathfrak{s l}(2, \mathbb{C})=\mathfrak{s o}(3, \mathbb{C})=\mathfrak{s p}(2, \mathbb{C}) \\
& \mathfrak{s o}(5, \mathbb{C})=\mathfrak{s p}(4, \mathbb{C}) \\
& \mathfrak{s l}(4, \mathbb{C})=\mathfrak{s o}(6, \mathbb{C}) \\
& \mathfrak{s o}(4, \mathbb{C})=\mathfrak{s l}(2, \mathbb{C}) \oplus \mathfrak{s l}(2, \mathbb{C}) \\
& \mathfrak{g l}(n, \mathbb{C})=\mathbb{C} \oplus \mathfrak{s l}(n, \mathbb{C})
\end{aligned}
$$

Estes isomorfismos devem ser levados em conta quando se aplicam os teoremas a seguir. Além disto, sempre que possível, indexaremos as séries infinitas de álgebras clássicas pelo seu posto $r$. No caso em que esta indexação for por outro parâmetro usaremos uma letra diferente.

\footnotetext{
${ }^{1}$ Mais exatamente, seria o conjunto das subalgebras redutivas maximais, conforme explicado anteriormente.
} 
Teorema 1.4.1 (Dynkin $[\mathbf{1 0}, \mathbf{1 1}]$ ). As subalgebras maximais redutíveis $\mathfrak{f}$ de uma álgebra de Lie clássica $\mathfrak{g}$ são conjugadas a uma, e somente uma, das subalgebras listadas na tabela 1.4.

\begin{tabular}{|c|c|c|c|}
\hline $\mathfrak{g}$ & Tipo & $\mathfrak{f}$ & $\operatorname{dim} \mathfrak{f}$ \\
\hline $\begin{array}{c}\mathfrak{s l}(r+1, \mathbb{C}) \\
r \geqslant 1\end{array}$ & I & $\begin{array}{c}\mathbb{C} \oplus \mathfrak{s l}(\ell, \mathbb{C}) \oplus \mathfrak{s l}(r-\ell+1, \mathbb{C}) \\
1 \leqslant \ell \leqslant[(r+1) / 2]\end{array}$ & $\begin{array}{l}(r-\ell)^{2}+\ell^{2}+ \\
\quad+2(r-\ell)\end{array}$ \\
\hline $\begin{array}{c}\mathfrak{s o}(2 r+1, \mathbb{C}) \\
r \geqslant 2\end{array}$ & II & $\begin{array}{c}\mathfrak{s o}(2 \ell, \mathbb{C}) \oplus \mathfrak{s o}(2(r-\ell)+1, \mathbb{C}) \\
1 \leqslant \ell \leqslant r\end{array}$ & $\begin{aligned} & 2(r-\ell)^{2}+ \\
&+ 2 \ell(\ell-1)+r \\
&\end{aligned}$ \\
\hline \multirow[t]{2}{*}{$\begin{array}{c}\mathfrak{s p}(2 r, \mathbb{C}) \\
r \geqslant 3\end{array}$} & III & $\begin{array}{c}\mathfrak{s p}(2 \ell, \mathbb{C}) \oplus \mathfrak{s p}(2(r-\ell), \mathbb{C}) \\
1 \leqslant \ell \leqslant[r / 2]\end{array}$ & $\begin{array}{l}2(r-\ell)^{2}+ \\
+2 \ell^{2}+r\end{array}$ \\
\hline & IV & $\mathbb{C} \oplus \mathfrak{s l}(r, \mathbb{C})=\mathfrak{g l}(r, \mathbb{C})$ & $r^{2}$ \\
\hline \multirow[t]{3}{*}{$\begin{array}{c}\mathfrak{s o}(2 r, \mathbb{C}) \\
r \geqslant 4\end{array}$} & $\mathrm{~V}$ & $\begin{array}{c}\mathfrak{s o}(2 \ell, \mathbb{C}) \oplus \mathfrak{s o}(2(r-\ell), \mathbb{C}) \\
1 \leqslant \ell \leqslant[r / 2]\end{array}$ & $\begin{array}{l}2(r-\ell)^{2}+ \\
+2 \ell^{2}-r\end{array}$ \\
\hline & VI & $\begin{array}{c}\mathfrak{s o}(2 \ell-1, \mathbb{C}) \oplus \mathfrak{s o}(2(r-\ell)+1, \mathbb{C}) \\
1 \leqslant \ell \leqslant[(r+1) / 2]\end{array}$ & $\begin{array}{l}2(r-\ell)^{2}+r+ \\
+(2 \ell-1)^{2}-\ell\end{array}$ \\
\hline & VII & $\mathbb{C} \oplus \mathfrak{s l}(r, \mathbb{C})=\mathfrak{g l}(r, \mathbb{C})$ & $r^{2}$ \\
\hline
\end{tabular}

Tabela 1.4: Subalgebras maximais redutíveis das álgebras de Lie clássicas.

\section{Observações.}

1. As subalgebras desta tabela se dividem naturalmente em duas categorias: ou são semisimples (tipo II quando $\ell>1$, tipo III, tipo V quando $\ell>1$, tipo VI) ou são soma direta de uma álgebra semisimples com uma álgebra unidimensional (tipo I, tipo II quando $\ell=1$, tipo IV, tipo $\mathrm{V}$ quando $\ell=1$, tipo VII). No segundo caso, a parte semisimples de $\mathfrak{f}$ é maximal dentre todas as subalgebras semisimples de $\mathfrak{g}$ quando $\mathfrak{f}$ for do tipo I, do tipo IV ou do tipo VII, mas não quando $\mathfrak{f}$ for do tipo II ou do tipo V com $\ell=1$, pois nestes casos,

$$
\begin{array}{lllccc}
\mathfrak{s o}(2 r-1, \mathbb{C}) & \subset & \mathfrak{s o}(2 r, \mathbb{C}) & \subset & \mathfrak{s o}(2 r+1, \mathbb{C}) & (\text { tipo II, } \ell=1) \\
\mathfrak{s o}(2 r-2, \mathbb{C}) & \subset & \mathfrak{s o}(2 r-1, \mathbb{C}) & \subset & \mathfrak{s o}(2 r, \mathbb{C}) & (\text { tipo V }, \ell=1)
\end{array}
$$


2. A inclusão de $\mathfrak{f}$ em $\mathfrak{g}$ é dada pela soma direta das representações fundamentais de cada ideal simples da subalgebra $\mathfrak{f}$. Mais exatamente, ela pode ser descrita da seguinte forma:

(a) Se $\mathfrak{g}$ for da forma $\mathfrak{s l}(p+q, \mathbb{C})$ e $\mathfrak{f}$ for da forma $\mathbb{C} \oplus \mathfrak{s l}(p, \mathbb{C}) \oplus \mathfrak{s l}(q, \mathbb{C})$, então a inclusão é da forma:

$$
\begin{aligned}
& \mathbb{C} \oplus \mathfrak{s l}(p, \mathbb{C}) \oplus \mathfrak{s l}(q, \mathbb{C}) \quad \longrightarrow \quad \mathfrak{s l}(p+q, \mathbb{C}) \\
& (\lambda, X, Y) \quad \longmapsto\left(\begin{array}{cc}
X+\frac{\lambda}{p} 1_{p} & 0 \\
0 & Y-\frac{\lambda}{q} 1_{q}
\end{array}\right)
\end{aligned}
$$

(b) Se $\mathfrak{g}$ for da forma $\mathfrak{s o}(p+q, \mathbb{C})$ e $\mathfrak{f}$ for da forma $\mathfrak{s o}(p, \mathbb{C}) \oplus \mathfrak{s} \mathfrak{o}(q, \mathbb{C})$, então a inclusão é da forma:

$$
\begin{aligned}
\mathfrak{s o}(p, \mathbb{C}) \oplus \mathfrak{s o}(q, \mathbb{C}) & \longrightarrow \mathfrak{s o}(p+q, \mathbb{C}) \\
(X, Y) & \longmapsto\left(\begin{array}{cc}
X & 0 \\
0 & Y
\end{array}\right)
\end{aligned}
$$

(c) Se $\mathfrak{g}$ for da forma $\mathfrak{s p}(2 p+2 q, \mathbb{C})$ e $\mathfrak{f}$ for da forma $\mathfrak{s p}(2 p, \mathbb{C}) \oplus \mathfrak{s p}(2 q, \mathbb{C})$, então a inclusão é da forma:

$$
\begin{array}{cl}
\mathfrak{s p}(2 p, \mathbb{C}) \oplus \mathfrak{s p}(2 q, \mathbb{C}) & \longrightarrow \mathfrak{s p}(2 p+2 q, \mathbb{C}) \\
(X, Y) & \longmapsto
\end{array}
$$

(d) Se $\mathfrak{g}$ for da forma $\mathfrak{s o}(2 p, \mathbb{C})$ e $\mathfrak{f}$ for da forma $\mathfrak{g l}(p, \mathbb{C})$, então a inclusão é da forma:

$$
\begin{aligned}
& \mathfrak{g l}(p, \mathbb{C}) \quad \longrightarrow \quad \mathfrak{s o}(2 p, \mathbb{C}) \\
& X \longmapsto\left(\begin{array}{cc}
X & 0 \\
0 & -X^{T}
\end{array}\right)
\end{aligned}
$$

(e) Se $\mathfrak{g}$ for da forma $\mathfrak{s} \mathfrak{p}(2 p, \mathbb{C})$ e $\mathfrak{f}$ for da forma $\mathfrak{g l}(p, \mathbb{C})$, então a inclusão é da forma:

$$
\begin{array}{clc}
\mathfrak{g l}(p, \mathbb{C}) & \longrightarrow & \mathfrak{s p}(2 p, \mathbb{C}) \\
X & \longmapsto & \left(\begin{array}{cc}
X & 0 \\
0 & -X^{T}
\end{array}\right)
\end{array}
$$

Nos dois últimos casos estamos usando a definição alternativa que demos para $\mathfrak{s o}(2 r, \mathbb{C})$ e $\mathfrak{s p}(2 r, \mathbb{C})$. Fica claro, agora que demos as inclusões explicitamente, que as subalgebras da tabela 4 são realmente redutíveis, na verdade são completamente redutíveis. 
3. As subalgebras desta tabela que são semisimples são maximais dentre todas as subalgebras de $\mathfrak{g}$ e não somente dentre as redutivas, enquanto que as que possuem centro não-trivial não o são. De fato, estão contidas em subalgebras não-redutivas chamadas subalgebras parabólicas maximais. Uma subalgebra $\mathfrak{p}$ de $\mathfrak{g}$ é chamada parabólica se contém alguma subalgebra de Borel de $\mathfrak{g}$ (isto é, uma subalgebra solúvel maximal). Segundo um teorema de Karpelevich ${ }^{1}$, qualquer subalgebra maximal não-semisimples de uma álgebra de Lie complexa semisimples é conjugada a uma subalgebra parabólica maximal. As classes de conjugação de subalgebras parabólicas maximais estão em correspondência biunívoca com as raizes simples de $\mathfrak{g}$. Pórem, estas subalgebras não possuem forma real compacta e portanto não fornecem subalgebras maximais quando passamos para a forma real compacta de $\mathfrak{g}$.

4. As subalgebras desta tabela correspondem a espaços simétricos riemannianos irredutíveis (ver Helgason [19, pag. 518, tab. V]), cuja classificação se deve a Cartan. Este problema se reduz à classificação de certas subalgebras das álgebras de Lie simples, fixadas por automorfismos involutivos (um automorfismo $\sigma$ de uma álgebra de Lie $\mathfrak{g}$ é involutivo se $\sigma \neq 1$ e $\sigma^{2}=1$ ). É um fato importante que todas estas subalgebras são maximais. As subalgebras do tipo I correspondem aos espaços simétricos do tipo $A I I I^{7}$, que são as grassmannianas complexas $S U(p+q) / \mathrm{S}(U(p) \times U(q))$, as subalgebras de tipo II, V e VI correspondem a uma única família de espaços simétricos, denotada por $B D I$, que são as grassmannianas reais $S O(p+q) / S O(p) \times S O(q)$, as subalgebras de tipo III correspondem aos espaços simétricos do tipo $C I I$, que são as grassmannianas quaterniônicas $S p(2 p+2 q) / S p(2 p) \times S p(2 q)$, e finalmente, as subalgebras de tipo IV correspondem aos espaços simétricos de tipo $C I$ e as subalgebras de tipo VII aos espaços simétricos de tipo D III que são os espaços simétricos $S p(2 n) / U(n)$ e $S O(2 n) / U(n)$ respectivamente: note que em todos estes exemplos a álgebra $\mathfrak{g}$ é simples e a a subalgebra f é semisimples mas não simples ou tem um centro unidimensional. Há ainda os espaços simétricos associados às álgebras excepcionais, e mais duas famílias, $A I$ e $A I I$, para as quais a subalgebra $\mathfrak{f}$ é simples e irredutível.

\footnotetext{
${ }^{1}$ Este teorema aparece pela primeira vez no artigo On nonsemisimple maximal subalgebras of semisimple Lie algebras, Dokl. Akad. Nauk SSSR 76 (1951), 775-778 (russo). Este artigo nunca foi traduzido para o inglês, mas uma demonstração do teorema pode ser encontrada em Golubitsky [16].

${ }^{7} \mathrm{~A}$ notação usada aqui é a mesma introduzida por Cartan na sua classificação dos espaços simétricos e que se tornou padrão desde então. O trabalho de Cartan em álgebras de Lie se iniciou com sua tese de doutorado, apresentada em 1894, onde é dada a classificação das álgebras de Lie simples complexas, depois ele se dedicou à classificação das álgebras de Lie simples reais obtida finalmente em 1914, a partir daí ele voltou seus esforços à teoria dos espaçoes simétricos, concluíndo sua classificação numa série de artigos entre os anos de 1926 e 1927. Este trabalho monumental foi simplificado e aperfeiçoado por vários matemáticos e constitui hoje uma das principais áreas da geometria. Uma apresentação moderna e completa, partindo de noções elementares e chegando à classificação dos espaços simétricos pode ser encontrada em [19].
} 
Teorema 1.4.2 (Dynkin [11]). As subalgebras maximais irredutíveis não simples $\mathfrak{f}$ de uma álgebra de Lie clássica $\mathfrak{g}$ são conjugadas a uma, e somente uma, das subalgebras listadas na tabela 1.5.

\begin{tabular}{|c|c|c|c|}
\hline $\mathfrak{g}$ & Tipo & $\mathfrak{f}$ & $\operatorname{dim} \mathfrak{f}$ \\
\hline $\mathfrak{s l}(n, \mathbb{C})$ & I & $\begin{array}{c}\mathfrak{s l}(s, \mathbb{C}) \times \mathfrak{s l}(t, \mathbb{C}) \\
n=s t ; s, t \geqslant 2\end{array}$ & $s^{2}+t^{2}-2$ \\
\hline \multirow[t]{2}{*}{$\mathfrak{s o}(n, \mathbb{C})$} & II & $\begin{array}{c}\mathfrak{s o}(s, \mathbb{C}) \times \mathfrak{s o}(t, \mathbb{C}) \\
n=s t ; s, t \geqslant 3 ; s, t \neq 4\end{array}$ & $\frac{1}{2}\left(s^{2}+t^{2}-s-t\right)$ \\
\hline & III & $\begin{array}{c}\mathfrak{s p}(s, \mathbb{C}) \times \mathfrak{s p}(t, \mathbb{C}) \\
n=s t ; s, t \geqslant 2\end{array}$ & $\frac{1}{2}\left(s^{2}+t^{2}+s+t\right)$ \\
\hline $\mathfrak{s p}(n, \mathbb{C})$ & IV & $\begin{array}{c}\mathfrak{s p}(s, \mathbb{C}) \times \mathfrak{s o}(t, \mathbb{C}) \\
n=s t ; s \geqslant 2, t \geqslant 3, t \neq 4 \\
\text { ou } \quad s=2, t=4\end{array}$ & $\frac{1}{2}\left(s^{2}+t^{2}+s-t\right)$ \\
\hline
\end{tabular}

Tabela 1.5: Subalgebras maximais irredutíveis não simples das álgebras de Lie clássicas.

\section{Observações.}

1. A inclusão de $\mathfrak{f}$ em $\mathfrak{g}$ é dada pelo produto tensorial das representações fundamentais de cada ideal simples da subalgebra $\mathfrak{f}$.

2. Os parâmetros $n, s$ e $t$ podem assumir quaisquer valores naturais que satisfazem as restrições indicadas na tabela, exceto no caso em que eles aparecem em uma álgebra simplética que, pelo fato de só admitir representação fundamental em dimensão par, exige que os parâmentros sejam números naturais pares.

3. Todas as subalgebras desta tabela são maximais não só dentre as subalgebras semisimples de $\mathfrak{g}$, mas dentre todas as subalgebras de $\mathfrak{g}$.

4. As subalgebras desta tabela só aparecem quando a dimensão da representação fundamental de $\mathfrak{g}$ for um número composto, e portanto a sua distribuição nas séries clássicas não é tão regular quanto a das subalgebras da tabela anterior.

5. Nenhuma subalgebra desta tabela fornece espaços simétricos riemannianos. 
Para enunciarmos o teorema de classificação das subalgebras maximais irredutíveis simples, precisaremos de mais algumas definições, pois este teorema difere dos anteriores quanto ao caráter do seu enunciado que lista as subalgebras que não são maximais em $\mathfrak{s l}(n, \mathbb{C}), \mathfrak{s o}(n, \mathbb{C})$ ou $\mathfrak{s p}(n, \mathbb{C})$.

Seja $\mathfrak{f}$ uma álgebra de Lie simples e fixemos uma representação irredutível $\rho$ de $\mathfrak{f}$ em um espaço vetorial complexo $V$ de dimensão $n$ com peso máximo $\Lambda$. Podemos considerar esta representação como uma inclusão de $\mathfrak{f} \operatorname{em~} \mathfrak{g l}(n, \mathbb{C})$, pois devido ao fato de $\mathfrak{f}$ ser simples, a representação $\rho$ é fiel. Devido ainda ao fato de $\mathfrak{f}$ ser simples temos que $[\rho(\mathfrak{f}), \rho(\mathfrak{f})]=\rho(\mathfrak{f})$, e portanto $\rho(\mathfrak{f}) \subset \mathfrak{s l}(n, \mathbb{C})$. Logo toda representação irredutível de uma álgebra de Lie simples $\mathfrak{f}$ é uma inclusão desta álgebra como subalgebra irredutível simples em $\mathfrak{s l}(n, \mathbb{C})$. Após esta observação, surgem imediatamente duas perguntas: (a) como saber se $\mathfrak{f}$ é uma subalgebra de $\mathfrak{s l}(n, \mathbb{C}), \mathfrak{s o}(n, \mathbb{C})$ ou $\mathfrak{s p}(n, \mathbb{C})$ e $(\mathrm{b})$ como saber quando duas inclusões da mesma álgebra $\mathfrak{f}$ são conjugadas em $\mathfrak{s l}(n, \mathbb{C}), \mathfrak{s o}(n, \mathbb{C})$ ou $\mathfrak{s p}(n, \mathbb{C})$. Estas perguntas foram respondidas por Malcev [34], que foi o precursor de Dynkin na classificação das subalgebras maximais das álgebras de Lie simples. A idéia é interpretar a inclusão de $\mathfrak{f}$ em $\mathfrak{s l}(n, \mathbb{C}), \mathfrak{s o}(n, \mathbb{C})$ ou $\mathfrak{s p}(n, \mathbb{C})$ como uma representação irredutível e reinterpretar as questões (a) e (b) como questões da teoria de representações de álgebras de Lie.

Para responder a estas duas perguntas observemos primeiramente que duas representações irredutíveis $\rho_{1}$ e $\rho_{2}$ de uma álgebra de Lie simples $\mathfrak{f}$ em um espaço vetorial $V$ são equivalentes se e somente se as subalgebras $\rho_{1}(\mathfrak{f})$ e $\rho_{2}(\mathfrak{f})$ são conjugadas em $\mathfrak{g l}(V)$. Lembremos também que a representação dual ou contragrediente $\rho^{*}: \mathfrak{f} \rightarrow \mathfrak{g l}\left(V^{*}\right)$ a uma representação $\rho: \mathfrak{f} \rightarrow \mathfrak{g l}(V)$ é definida por

$$
\left\langle\rho^{*}(X) v^{*}, v\right\rangle=-\left\langle v^{*}, \rho(X) v\right\rangle
$$

para todo $X \in \mathfrak{f}, v^{*} \in V^{*}$ e $v \in V$. Dizemos que uma representação $\rho$ é auto-dual ou autoconjugada se $\rho$ for equivalente a $\rho^{*}$. Isto significa que existe um isomorfismo equivariante entre $V$ e $V^{*}$, ou equivalentemente, existe uma forma bilinear não-degenerada $\rho$-invariante sobre $V$. Dizemos que $\rho$ é ortogonal se existe uma forma bilinear simétrica sobre $V$ invariante por $\rho$. Similarmente $\rho$ é chamada simplética se existe uma forma bilinear anti-simétrica sobre $V$ invariante por $\rho$. Uma outra forma de expressar isto é dizer que $\rho(X)$ pertence à subalgebra ortogonal/simplética definida por esta forma simétrica/anti-simétrica, para todo $X \in \mathfrak{f}$. Por outro lado, é possível garantir que uma forma bilinear $\rho$-invariante sobre $V$ é não-degenerada ou nula, é única a menos de uma constante não-nula e é automáticamente simétrica ou anti-simétrica. Portanto, dizer que $\mathfrak{f}$ é uma subalgebra de $\mathfrak{s o}(n, \mathbb{C}) / \mathfrak{s p}(n, \mathbb{C})$ é o mesmo que dizer que a inclusão de $\mathfrak{f} \operatorname{em~} \mathfrak{s l}(n, \mathbb{C})$, quando vista como uma representação linear em $\mathbb{C}^{n}$, é ortogonal/simplética. Podemos introduzir a seguinte notação: se $\rho$ for ortogonal diremos que $\mathfrak{f}$ é do tipo $1(\mathfrak{f} \subset \mathfrak{s o}(n, \mathbb{C}))$, se $\rho$ for simplética diremos que $\mathfrak{f}$ é do tipo -1 $(\mathfrak{f} \subset \mathfrak{s p}(n, \mathbb{C}))$ e se $\rho$ não for auto-dual diremos que $\mathfrak{f}$ é do tipo $0(\mathfrak{f} \subset \mathfrak{s l}(n, \mathbb{C}))$. 
Nestes termos, a resposta à pergunta (a) é dada pelo seguinte teorema, originalmente devido a Malcev [34] e subsequentemente aperfeiçoado por Dynkin [11]; uma demonstração pode ser encontrada em Samelson [45, pag. 140] ${ }^{1}$.

Teorema 1.4.3. Seja $\mathfrak{f}$ uma álgebra de Lie simples e $\rho_{\Lambda}$ uma representação irredutível de $\mathfrak{f}$ com peso máximo

$$
\Lambda=\sum_{i=1}^{r} p_{i} \lambda_{i}
$$

Supondo que os números $p_{i}$ estejam indexados de acordo com a tabela 1.1, temos as seguintes possibilidades para $\mathfrak{f}$ :

(i) $\mathfrak{f}=\mathfrak{s l}(r+1, \mathbb{C}): \rho_{\Lambda}$ é auto-dual se e somente se $p_{i}=p_{r-i+1}(i=1, \ldots, r)$; em particular, é sempre auto-dual quando $r=1$. Nestes casos $\rho_{\Lambda}$ é simplética quando $r=1(\bmod 4)$ e $p_{(r+1) / 2}$ é impar e é ortogonal caso contrário;

(ii) $\mathfrak{f}=\mathfrak{s o}(2 r+1, \mathbb{C}): \rho_{\Lambda}$ é sempre auto-dual. Então $\rho_{\Lambda}$ é simplética quando $r=1(\bmod 4)$ ou $r=2(\bmod 4)$ e $p_{r}$ é impar e é ortogonal caso contrário;

(iii) $\mathfrak{f}=\mathfrak{s p}(2 r, \mathbb{C}): \rho_{\Lambda}$ é sempre auto-dual. Então $\rho_{\Lambda}$ é simplética quando $p_{1}+p_{3}+p_{5}+\ldots$ é impar e é ortogonal caso contrário;

(iv) $\mathfrak{f}=\mathfrak{s o}(2 r, \mathbb{C}): \rho_{\Lambda}$ é auto-dual se e somente se $r$ é par, ou $r$ é impar e $p_{r-1}=p_{r}$. Nestes casos $\rho_{\Lambda}$ é simplética quando $r=1(\bmod 4)$ e $p_{r-1}+p_{r}$ é impar e é ortogonal caso contrário;

(v) $\mathfrak{f}=G_{2}: \rho_{\Lambda}$ é sempre auto-dual e ortogonal para todo $\Lambda$;

(vi) $\mathfrak{f}=F_{4}: \rho_{\Lambda}$ é sempre auto-dual e ortogonal para todo $\Lambda$;

(vii) $\mathfrak{f}=E_{6}: \rho_{\Lambda}$ é auto-dual se e somente se $p_{1}=p_{5}$ e $p_{2}=p_{4}$. Nestes casos $\rho_{\Lambda}$ é sempre ortogonal;

(viii) $\mathfrak{f}=E_{7}: \rho_{\Lambda}$ é sempre auto-dual. Então $\rho_{\Lambda}$ é simplética quando $p_{1}+p_{3}+p_{7}$ é impar e é ortogonal caso contrário;

(ix) $\mathfrak{f}=E_{8}: \rho_{\Lambda}$ é sempre auto-dual e ortogonal para todo $\Lambda$.

\footnotetext{
${ }^{1}$ Quando $\Lambda$ é o peso máximo de uma representação auto-dual $\rho_{\Lambda}$ a altura (ver Dynkin [11]) de $\Lambda$, denotada por $\operatorname{ht}(\Lambda)$, permite decidir se a representação $\rho_{\Lambda}$ é ortogonal ou simplética: ela é ortogonal se e somente se ht $(\Lambda)$ for par e é simplética se e somente se ht $(\Lambda)$ for impar. O teorema 1.4.3 mostra então como calcular a altura do peso máximo associado a uma representação auto-dual $\rho_{\Lambda}$ para cada álgebra de Lie simples. Com esta noção podemos definir o "tipo" de uma representação auto-dual $\rho_{\Lambda}$ como sendo igual a $(-1)^{\mathrm{ht}(\Lambda)}$.
} 
No mesmo trabalho de Malcev [34] encontra-se a resposta à pergunta (b), resolvendo assim o problema de conjugação entre duas subalgebras irredutíveis simples. Uma demonstração se encontra em Onishchik \& Vinberg [42, pag. 204, teo. 3.2].

Teorema 1.4.4. Seja $\mathfrak{f}$ uma subalgebra qualquer de uma álgebra de Lie clássica $\mathfrak{g}$ e sejam $\varphi, \psi: \mathfrak{f} \rightarrow \mathfrak{g}$ duas inclusões. Então temos as seguintes possibilidades:

(i) $\mathfrak{g}=\mathfrak{s l}(r+1, \mathbb{C})$. Se $\varphi$ e $\psi$ são conjugadas em $\mathfrak{s l}(r+1, \mathbb{C})$ então $\varphi$ e $\psi$ são equivalentes como representações lineares. Reciprocamente, se $\varphi$ e $\psi$ são equivalentes como representações lineares então $\varphi$ e $\psi$ são conjugadas. Ademais se $\varphi$ e $\psi^{*}$ são equivalentes como representações lineares, então existe um automorfismo externo $\sigma$ de $\mathfrak{s l}(r+1, \mathbb{C})$ tal que $\varphi=\sigma \circ \psi$.

(ii) $\mathfrak{g}=\mathfrak{s o}(2 r+1, \mathbb{C})$. Então $\varphi$ e $\psi$ são conjugadas se e somente se são equivalentes como representações lineares.

(iii) $\mathfrak{g}=\mathfrak{s p}(2 r, \mathbb{C})$. Então $\varphi$ e $\psi$ são conjugadas se e somente se são equivalentes como representações lineares.

(iv) $\mathfrak{g}=\mathfrak{s o}(2 r, \mathbb{C})$. Se $\varphi$ e $\psi$ são conjugadas então $\varphi$ e $\psi$ são equivalentes como representações lineares. Reciprocamente, se $\varphi$ e $\psi$ são equivalentes como representações lineares, então existe um automorfismo $\sigma$ de $\mathfrak{s o}(2 r, \mathbb{C})$ tal que $\varphi=\sigma \circ \psi$, que pode ser interno ou externo. Se ele for interno então as inclusões são conjugadas.

Finalmente podemos enunciar o principal teorema do trabalho de Dynkin sobre a classificação das subalgebras maximais das álgebras clássicas. Como já foi dito este teorema pode ser interpretado da seguinte forma: Quase todas as subalgebras irredutíveis simples são maximais em $\mathfrak{s l}(n, \mathbb{C}), \mathfrak{s o}(n, \mathbb{C})$ ou $\mathfrak{s p}(n, \mathbb{C})$, sendo que as exeções a esta afirmação estão listadas em uma tabela. Nesta tabela, os casos excepcionais são indicados listando a álgebra simples $\mathfrak{f}$, o peso máximo $\Lambda$ da representação irredutível de $\mathfrak{f}$, a dimensão $n=\operatorname{dim} V_{\Lambda}$ desta representação e, finalmente, o tipo desta representação, conforme a notação introduzida acima:

$$
\begin{aligned}
\text { tipo } & \Rightarrow \mathfrak{g}=\mathfrak{s l}(n, \mathbb{C}) \\
\text { tipo } 1 & \Rightarrow \mathfrak{g}=\mathfrak{s o}(n, \mathbb{C}) \\
\text { tipo }-1 & \Rightarrow \mathfrak{g}=\mathfrak{s p}(n, \mathbb{C}) \quad(n \text { par })
\end{aligned}
$$

A dimensão $n=\operatorname{dim} V_{\Lambda}$ é calculada pela fórmula de Weyl

$$
\operatorname{dim} V_{\Lambda}=\prod_{\alpha \in \Delta^{+}} \frac{(\Lambda+\varrho, \alpha)}{(\varrho, \alpha)}
$$

onde $\Delta^{+}$é o conjunto das raizes positivas e

$$
\varrho=\frac{1}{2} \sum_{\alpha \in \Delta^{+}} \alpha .
$$


Teorema 1.4.5 (Dynkin [11]). As subalgebras irredutiveis simples $\mathfrak{f}$ de uma álgebra de Lie clássica $\mathfrak{g}$, dadas por uma representação irredutível de $\mathfrak{f}$ com peso máximo $\Lambda$, são maximais, exceto quando $\mathfrak{f}$ e $\Lambda$ correspondem a um dos casos excepcionais listados na tabela 1.6.

\begin{tabular}{|c|c|c|c|}
\hline $\mathfrak{f}$ & $\Lambda$ & $n=\operatorname{dim} V_{\Lambda}$ & Tipo \\
\hline $\mathfrak{s l}(r+1, \mathbb{C}) \quad(r \geqslant 3)$ & $\lambda_{1}+\lambda_{3}$ & $3\left(\begin{array}{c}r+2 \\
4\end{array}\right)$ & 0 \\
\hline $\mathfrak{s l}(r+1, \mathbb{C}) \quad(r \geqslant 2)$ & $2 \lambda_{1}+\lambda_{2}$ & $3\left(\begin{array}{c}r+3 \\
4\end{array}\right)$ & 0 \\
\hline $\mathfrak{s l}(2, \mathbb{C})$ & $6 \lambda_{1}$ & 7 & 1 \\
\hline $\mathfrak{s l}(6, \mathbb{C})$ & $\lambda_{2}+\lambda_{4}$ & 189 & 1 \\
\hline $\mathfrak{s o}(4 \ell+3, \mathbb{C}) \quad(\ell \geqslant 1)$ & $k \lambda_{2 \ell+1} \quad(k \geqslant 2)$ & $\prod_{i=1}^{2 \ell+1} \frac{\left(\begin{array}{c}k+2 i-1 \\
k\end{array}\right)}{\left(\begin{array}{c}k+i-1 \\
k\end{array}\right)}$ & $(-1)^{(\ell+1) k}$ \\
\hline $\mathfrak{s o}(9, \mathbb{C})$ & $\lambda_{1}+\lambda_{4}$ & 128 & 1 \\
\hline $\mathfrak{s p}(6, \mathbb{C})$ & $\begin{array}{c}2 \lambda_{2} \\
2 \lambda_{2}+\lambda_{3}\end{array}$ & $\begin{array}{c}90 \\
350\end{array}$ & $\begin{array}{r}1 \\
-1 \\
\end{array}$ \\
\hline $\mathfrak{s o}(10, \mathbb{C})$ & $\lambda_{2}+\lambda_{4}$ & 560 & 0 \\
\hline $\mathfrak{s o}(12, \mathbb{C})$ & $\begin{array}{c}\lambda_{4} \\
\lambda_{3}+\lambda_{5}\end{array}$ & $\begin{array}{r}495 \\
4928 \\
\end{array}$ & $\begin{array}{r}1 \\
-1 \\
\end{array}$ \\
\hline$E_{6}$ & $\begin{array}{c}\lambda_{2} \\
\lambda_{4}+\lambda_{6}\end{array}$ & $\begin{array}{c}351 \\
17550\end{array}$ & $\begin{array}{l}0 \\
0 \\
\end{array}$ \\
\hline$E_{7}$ & $\begin{array}{c}\lambda_{2} \\
\lambda_{3} \\
\lambda_{4} \\
\lambda_{5}+\lambda_{7}\end{array}$ & $\begin{array}{c}1539 \\
27664 \\
365750 \\
3792096 \\
\end{array}$ & $\begin{array}{r}1 \\
-1 \\
1 \\
-1 \\
\end{array}$ \\
\hline$G_{2}$ & $k \lambda_{1} \quad(k \geqslant 2)$ & $\frac{2 k+5}{5}\left(\begin{array}{c}k+4 \\
4\end{array}\right)$ & 1 \\
\hline
\end{tabular}

Tabela 1.6: Subalgebras irredutíveis simples não maximais em $\mathfrak{s l}(n, \mathbb{C})($ tipo 0$), \mathfrak{s o}(n, \mathbb{C})($ tipo 1$)$ ou $\mathfrak{s p}(n, \mathbb{C}), n$ par $($ tipo -1$)$. 


\section{Observações.}

1. Há duas séries de subalgebras maximais irredutíveis simples, com $\mathfrak{g}=\mathfrak{s l}(n, \mathbb{C})$, dadas pelas inclusões naturais $\mathfrak{s o}(n, \mathbb{C}) \subset \mathfrak{s l}(n, \mathbb{C})$ e $\mathfrak{s p}(n, \mathbb{C}) \subset \mathfrak{s l}(n, \mathbb{C})$. Estas são as únicas sequências de subalgebras maximais irredutíveis simples que seguem um padrão em uma série de álgebras clássicas, enquanto que as outras possuem uma distribuição muito irregular. Além disto estas duas inclusões correspondem exatamente aos espaços riemannianos simétricos irredutíveis dados pelas famílias $A I$ e $A I I$ que correspondem aos espaços $S U(2 n) / S O(n)$ e $S U(2 n) / S p(n)$.

2. O caso de subalgebras irredutíveis simples é o único em que as álgebras excepcionais aparecem como subalgebras maximais dentro de alguma álgebra clássica. Outro fato curioso é que nenhuma inclusão maximal de uma álgebra excepcional fornece espaços simétricos riemannianos irredutíveis; porém, como veremos adiante, certas inclusões maximais de álgebras clássicas em álgebras excepcionais definem os espaços riemannianos simétricos irredutíveis que ainda não apareceram.

Com o conjunto de teoremas acima, temos um método completo para classificar as subalgebras maximais simples irredutíveis de uma álgebra de Lie clássica $\mathfrak{g}$. A estratégia é a seguinte:

1. Determinar quais álgebras de Lie simples possuem representações irredutíveis com a mesma dimensão que a representação fundamental de $\mathfrak{g}$;

2. Usar o teorema 1.4.3 para decidir se as subalgebras encontradas são realmente subalgebras de $\mathfrak{g}$;

3. Consultar a tabela 1.6 para eliminar as subalgebras que não são maximais;

4. Aplicar o teorema 1.4.4 para eliminar as redundâncias, obtendo assim o conjunto das classes de conjugação das subalgebras irredutíveis simples maximais.

O último teorema a ser enunciado também é devido a Dynkin: ele classifica completamente as subalgebras redutivas maximais das álgebras excepcionais. Neste caso daremos uma tabela com todas as possíveis classes de conjugação (ou $L$-conjugação) de subalgebras, indicando subalgebras que são isomorfas como álgebras abstratas mas não são subalgebras conjugadas com um índice superior entre parênteses. Por exemplo, a álgebra $E_{7}$ possui duas subalgebras maximais $A_{1}^{(1)}$ e $A_{1}^{(2)}$ que não são conjugadas em $E_{7}$, apesar de serem isomorfas. No caso das álgebras excepcionais este fenômeno só ocorre com as subalgebras de tipo $A_{1}$. 
Teorema 1.4.6 (Dynkin [10]). Seja $\mathfrak{f}$ uma subalgebra redutiva maximal de uma álgebra de Lie excepcional $\mathfrak{g}$. Então $\mathfrak{f}$ é conjugada a uma das subalgebras da segunda coluna ou linearmente conjugada a uma das subalgebras da terceira coluna da tabela 1.7.

\begin{tabular}{|c|c|c|}
\hline $\begin{array}{c}\text { Álgebra } \\
\text { Excepcional }\end{array}$ & $\begin{array}{c}\text { Subalgebras de } \\
\text { Posto Máximo }\end{array}$ & $\begin{array}{c}\text { Subalgebras } \\
\text { Singulares }\end{array}$ \\
\hline \hline$E_{6}$ & $A_{1} \oplus A_{5}$ & $A_{1}$ \\
& $A_{2} \oplus A_{2} \oplus A_{2}$ & $C_{4}$ \\
& $\mathbb{C} \oplus D_{5}$ & $F_{4}$ \\
& & $G_{2}$ \\
& & $A_{2} \oplus G_{2}$ \\
\hline$E_{7}$ & $A_{7}$ & $A_{1}^{(1)}$ \\
& $A_{1} \oplus D_{6}$ & $A_{1}^{(2)}$ \\
& $A_{2} \oplus A_{5}$ & $A_{2}$ \\
& $\mathbb{C} \oplus E_{6}$ & $A_{1} \oplus A_{1}$ \\
& & $A_{1} \oplus F_{4}$ \\
& & $A_{1} \oplus G_{2}$ \\
& & $C_{3} \oplus G_{2}$ \\
\hline$E_{8}$ & $A_{8}$ & $A_{1}^{(1)}$ \\
& $D_{8}$ & $A_{1}^{(2)}$ \\
& $A_{1} \oplus E_{7}$ & $A_{1}^{(3)}$ \\
& $A_{2} \oplus E_{6}$ & $B_{2}$ \\
& $A_{4} \oplus A_{4}$ & $A_{1} \oplus A_{1}$ \\
& & $F_{4} \oplus G_{2}$ \\
\hline$F_{4}$ & $B_{4}$ & $A_{1}$ \\
& $A_{1} \oplus C_{3}$ & $A_{1} \oplus G_{2}$ \\
& $A_{2} \oplus A_{2}$ & \\
\hline$G_{2}$ & $A_{2}$ & $A_{1}$ \\
& $A_{1} \oplus A_{1}$ & \\
\hline & & \\
& & \\
& & \\
& & \\
& & \\
& & \\
& & \\
& & \\
& & \\
& & \\
& & \\
& &
\end{tabular}

Tabela 1.7: Subalgebras maximais das álgebras de Lie excepcionais. 
Observações. A segunda coluna da tabela 1.7 lista as subalgebras maximais de posto máximo (uma subalgebra $\mathfrak{f}$ de uma álgebra de Lie $\mathfrak{g}$ é dita de posto máximo se existe uma subalgebra de $\operatorname{Cartan}^{1} \mathfrak{h}$ de $\mathfrak{g}$ tal que $\mathfrak{h} \subset \mathfrak{f}$ ). Vale observar que todas as subalgebras desta tabela que possuem centro não-trivial são de posto máximo. A terceira coluna se refere as subalgebras singulares ou $S$-subalgebras, isto é, subalgebras que não estão contidas em nenhuma subalgebra regular própria de $\mathfrak{g}$ (uma subalgebra $\mathfrak{f}$ de uma álgebra de Lie $\mathfrak{g}$ é dita regular se o seu normalizador $\mathfrak{n}_{\mathfrak{g}}(\mathfrak{f})$ contém alguma subalgebra de Cartan de $\mathfrak{g}$ ). Pode-se provar que todas as subalgebras singulares são semisimples; na verdade, as propriedades das subalgebras singulares são similares às das subalgebras irredutíveis das álgebras clássicas. Note que, somente na coluna de subalgebras singulares, aparecem subalgebras que são isomorfas como álgebras de Lie abstratas, mas não são conjugadas; de fato, as subalgebras singulares são classificadas apenas a menos de conjugação linear, isto é, existem mais classes de conjugação do que a tabela indica. Porém isto não é grave, pois as regras de ramificação só dependem das classes de conjugação linear. Por fim, podemos associar a algumas subalgebras da tabela 1.7 certos espaços simétricos riemannianos irredutíveis, da mesma forma como para as álgebras clássicas, mas nem todas as subalgebras desta lista estão associadas a espaços simétricos. Na tabela 1.8 apresentamos uma lista completa (extraída de Helgason [19, pag. 518, tab. V]) dos espaços simétricos associados às álgebras excepcionais.

\begin{tabular}{|c|c|c|}
\hline $\begin{array}{c}\text { Álgebra } \\
\text { Excepcional }\end{array}$ & $\begin{array}{c}\text { Subalgebras } \\
\text { Maximais }\end{array}$ & $\begin{array}{c}\text { Notação } \\
\text { de Cartan }\end{array}$ \\
\hline \hline$E_{6}$ & $C_{4}$ & $E I$ \\
& $A_{1} \oplus A_{5}$ & $E I I$ \\
& $\mathbb{C} \oplus D_{5}$ & $E I I I$ \\
& $F_{4}$ & $E I V$ \\
\hline$E_{7}$ & $A_{7}$ & $E V$ \\
& $A_{1} \oplus D_{6}$ & $E V I$ \\
& $\mathbb{C} \oplus E_{6}$ & $E V I I$ \\
\hline$E_{8}$ & $D_{8}$ & $E V I I I$ \\
& $A_{1} \oplus E_{7}$ & $E I X$ \\
\hline$F_{4}$ & $A_{1} \oplus C_{3}$ & $F I$ \\
& $B_{4}$ & $F I I$ \\
\hline$G_{2}$ & $A_{1} \oplus A_{1}$ & $G$ \\
\hline
\end{tabular}

Tabela 1.8: Espaços simétricos riemannianos associados às álgebras de Lie excepcionais.

\footnotetext{
${ }^{1}$ Lembremos que uma subalgebra de Cartan de uma álgebra de Lie redutiva é, por definição, a soma direta de seu centro e de uma subalgebra de Cartan de sua parte semisimples.
} 
Agora que temos uma visão completa da estrutura das subalgebras maximais das álgebras simples complexas, podemos fazer algumas observações de caráter global:

1. Examinando todas as tabelas que contêm subalgebras percebe-se que as álgebras com centro não-trivial aparecem somente na tabela 1.4 ou na tabela 1.7 e que todas possuem centro unidimensional e correspondem a espaços simétricos, que na verdade são exatamente os espaços simétricos hermitianos. Então temos uma correspondência biunívoca entre os espaços simétricos hermitianos e as subalgebras maximais redutivas com centro não-trivial.

2. Podemos também listar as subalgebras de posto máximo, que aparecem classificadas pela primeira vez num trabalho de Borel e Siebenthal [4]: todas as subalgebras da tabela 1.4, com exeção das do tipo VI, e todas as subalgebras da primeira coluna da tabela 1.7, e somente estas, são subalgebras de posto máximo.

3. Um outro fato interessante é que todas as subalgebras semisimples que listamos são maximais dentre todas as subalgebras e não somente dentre as subalgebras redutivas. Como observamos no caso das álgebras clássicas, as subalgebras maximais que possuem centro não-trivial estão contidas em subalgebras parabólicas maximais: isto também vale para as subalgebras redutivas das álgebras excepcionais.

4. Por fim, gostariamos de mencionar que no trabalho original de Dynkin há uma tabela que lista as subalgebras maximais de posto máximo das álgebras excepcionais, e que contém erros, pois aparecem subalgebras que na verdade não são maximais (para mais detalhes ver [25]). Estes erros foram observados e corrigidos por Tits [53] e Golubitsky \& Rothschild [17].

Mostramos na seção 2.3 como usar a classificação das subalgebras redutivas maximais das álgebras de Lie complexas simples para obter a classificação das subalgebras maximais das álgebras de Lie compactas, e gostariamos agora de indicar como se classificam as subalgebras maximais das outras formas reais das álgebras de Lie complexas simples. Consideremos primeiro o caso mais simples: uma álgebra complexa $\mathfrak{g}$ pode ser vista como uma álgebra real $\mathfrak{g}^{\mathbb{R}}$ se simplesmente "esquecermos" sua estrutura complexa: neste caso, Dynkin [11, pag. 256, teo. 1.6] demonstra que as subalgebras maximais de $\mathfrak{g}^{\mathbb{R}}$ são as subalgebras do tipo $\mathfrak{f}^{\mathbb{R}}$, onde $\mathfrak{f}$ é uma subalgebra complexa maximal de $\mathfrak{g}$, juntamente com todas as formas reais de $\mathfrak{g}$. Um outro caso relativamente simples é o da forma real normal que, como a forma real compacta, é única para cada álgebra complexa simples: as subalgebras semisimples maximais da forma normal de $\mathfrak{g}$ são simplesmente as formas normais das subalgebras maximais complexas semisimples de $\mathfrak{g}$. A diferença em relação ao caso compacto é que aqui existem certas subalgebras não-semisimples cujas complexificações são subalgebras parabólicas de $\mathfrak{g}$ : 
subalgebras com esta propriedade são chamadas subalgebras parabólicas reais, e o fato importante é que há uma correspondência biunívoca entre subalgebras parabólicas complexas e reais quando a forma real é normal. Portanto a classificação das subalgebras maximais das formas reais normais também segue do caso complexo. Outra diferença entre a forma normal e a forma compacta é que na forma real normal, assim como na sua complexificação, as subalgebras redutivas com centro não-trivial não são maximais dentre todas as subalgebras, pois estão contidas em subalgebras parabólicas reais maximais. Finalmente as subalgebras maximais das outras formas reais das álgebras de Lie complexas simples também foram classificadas, mas não é possível explicar o resultado de forma simples, e devemos nos restringir aqui a indicar as referências pertinentes: a classificação das subalgebras semisimples se encontra em Taufik [52] e Komrakov [30, 31] enquanto que a classsificação das subalgebras não-semisimples pode ser encontrada em Mostow [38] e Tao Huei-min [51].

No próximo capítulo faremos uso dos teoremas de classificação de Dynkin para determinar todas as subalgebras maximais das álgebras de Lie que possuem representações irredutíveis de dimensão 64 (uma lista completa das álgebras de Lie simples com esta propriedade é dada em [23]) e portanto podem reproduzir as degenerescências observadas no código genético. Deste modo mostraremos que as tabelas de McKay \& Patera [35], que contém todas as subalgebras semisimples maximais das álgebras de Lie simples de posto menor ou igual a $8^{1}$, fornecem essencialmente a lista completa dos subgrupos maximais conexos fechados dos grupos de Lie compactos simples. De fato, o teorema 1.3.4 permite transferir os teoremas de classificação de Dynkin para as álgebras de Lie compactas e, além disto, as regras de ramificação são preservadas tanto pela complexificação quanto pela restrição à forma compacta. Assim podemos interpretar as tabelas de McKay \& Patera [35] como se referindo às álgebras compactas ou complexas, conforme nos seja mais conveniente. Finalmente, o teorema clássico de correspondência entre subalgebras e subgrupos conexos [56, pags. 94, 95] diz que existe uma correspondência biunívoca entre os subgrupos de Lie conexos de um grupo de Lie $G$ e as subalgebras de sua álgebra de Lie $\mathfrak{g}$ e portanto pode-se passar das álgebras compactas para os grupos compactos de modo que as cadeias descendentes de subalgebras correspondam a cadeias descendentes de subgrupos conexos fechados ${ }^{7}$.

\footnotetext{
${ }^{1} \mathrm{Na}$ verdade, contém todas as subalgebras redutivas maximais, sendo que no caso das subalgebras que possuem centro não-trivial aparece apenas a parte semisimples desde que esta continue sendo maximal (ver observações após o teorema 1.4.1). Isto ocorre porque o centro não contribui no cálculo das regras de ramificação.

${ }^{7} \mathrm{O}$ fato de que qualquer subgrupo semisimples de um grupo de Lie compacto é fechado é um resultado não-trivial devido a Mostow [37, pag. 615, cor. 2].
} 
CAPÍtulo 2

\section{Quebra de Simetrias no Código Genético}

Neste capítulo formularemos um quadro geral para se construir modelos algébricos baseados no princípio de simetrias quebradas e o aplicaremos para obter modelos algébricos para o código genético, segundo a estratégia originalmente proposta por Hornos \& Hornos [22] e ligeiramente generalizada por Forger, Hornos \& Hornos [14].

\subsection{Modelo Geral de Quebra de Simetrias}

Seja $G$ um grupo de Lie compacto conexo e $\rho$ uma representação irredutível de $G$ em um espaço vetorial $V$. Quando restringirmos esta representação de $G$ a um subgrupo fechado $H$ de $G$, o espaço vetorial $V$ se decompõe numa soma direta de subespaços invariantes sob $H$ tal que $\rho$ se decompõe num conjunto de representações irredutíveis de $H$ nestes subespaços, segundo o que é denominado a regra de ramificação pertinente ${ }^{1}$. As vezes chamaremos estes subespaços invariantes de multipletos e o número de vezes que um subespaço invariante de uma dimensão fixada aparece é chamado de multiplicidade ${ }^{7}$ do multipleto. Quando nos referimos a um subespaço invariante em particular podemos especializar a nomenclatura de modo a especificar sua dimensão: um singleto representa um subespaço invariante de dimensão 1, um dubleto um de dimensão 2, um tripleto um de dimensão 3, e assim em diante.

\footnotetext{
${ }^{1}$ Se $H$ for um subgrupo de Lie não fechado, $H$ será substituído por seu fecho $\bar{H}$, pois a restrição de uma representação irredutível de $\bar{H}$ a $H$ será sempre uma representação irredutível de $H$ e portanto as regras de ramificação de $G$ a $\bar{H}$ e de $G$ a $H$ são as mesmas.

${ }^{7}$ Há uma certa confusão na literatura, onde a palavra "multiplicidade" é muitas vezes usada ao invés de "dimensão". Como precisaremos dos dois conceitos, devemos fazer uma clara distinção entre ambos.
} 
A idéia fundamental por trás dos modelos baseados em simetrias quebradas é de que as degenerescências encontradas em situações experimentais refletam uma simetria que é residual no sentido de que, originalmente, houve uma simetria maior que foi perdida, ou quebrada, ao longo de um processo de evolução: o grupo $G$ descreve a simetria original e o subgrupo $H$ a simetria residual que permanece após a quebra. Em termos de representações, houve originalmente um único multipleto sob $G$ que foi quebrado em vários multipletos sob $H$. Ademais, este processo pode ocorrer em várias fases distintas, o que leva à idéia de considerar cadeias de subgrupos fechados do tipo $G=H_{0} \supset H_{1} \supset \ldots \supset H_{k}=H$, junto com as sucessivas regras de ramificação de uma dada representação irredutível de $G$. Reciprocamente, podemos imaginar o problema inverso, isto é, algum fenômeno produz uma certa distribuição de multipletos (onde usamos a palavra multipleto num sentido livre, significando apenas um conjunto finito de dados agrupados em certas classes) e queremos reconstruir um processo que gerou esta distribuição de multipletos, supondo que ela foi obtida através de um processo de evolução que partiu de uma situação totalmente simétrica e que ao decorrer do tempo esta simetria foi parcialmente perdida. Segundo esta filosofia para construir um modelo concreto usando a teoria dos grupos de Lie compactos conexos precisamos escolher:

- um grupo de Lie compacto conexo simples $G$;

- uma representação irredutível de $G$ em um espaço vetorial $V$ cuja dimensão seja igual à soma das dimensões dos multipletos observados;

- uma cadeia $G=H_{0} \supset H_{1} \supset \ldots \supset H_{k}=H$ de subgrupos fechados conexos maximais, tal que a distribuição de multipletos obtida da representação de $G$ em $V$ por restrição ao último subgrupo $H$ da cadeia e subsequente decomposição em representações irredutíveis de $H$ seja igual à distribuição de multipletos observada.

A imposição de que $G$ seja simples é motivada pelo fato de que um grupo de Lie compacto conexo $G$ qualquer é um objeto composto: $G$ se decompõe unicamente no produto localmente direto $^{1}$ de subgrupos normais $G_{0}, G_{1}, \ldots, G_{k}$, onde $G_{0}$ é isomorfo a um toro e $G_{1}, \ldots, G_{k}$ são simples. Do mesmo modo exigimos que a representação de $G$ em $V$ seja irredutível: uma representação redutível significaria que já houve uma quebra de simetria anterior. Por outro lado, a restrição a grupos conexos é imposta por motivos técnicos: a extensão do programa a grupos compactos mas não necessariamente conexos constitui um problema ainda em aberto. Finalmente, a utilização de subgrupos maximais é apenas por conveniência: se por acaso tivessemos uma cadeia descendente de subgrupos não necessariamente maximais basta acrescentar a esta cadeia todos os subgrupos intermediários, obtendo assim uma nova cadeia que satisfaz a condição de maximalidade e produz o mesmo resultado que a cadeia original.

\footnotetext{
${ }^{1}$ Dizemos que um grupo de Lie $G$ se decompõe num produto localmente direto de subgrupos normais $G_{1}, \ldots, G_{k}$ se $G=G_{1} \ldots G_{k}$ e todas as intersecções $G_{i} \cap\left(G_{1} \ldots G_{i-1} G_{i+1} \ldots G_{k}\right)(i=1, \ldots, k)$ são discretas.
} 
Há vários motivos para considerar o problema de construir um modelo algébrico para a evolução do código genético dentro da categoria dos grupos de Lie compactos. Em primeiro lugar, grupos de Lie compactos formam uma classe natural de objetos, sendo uma "versão contínua" dos grupos finitos, que aliás são uma outra classe natural de objetos na qual o mesmo problema merece ser investigado [22]. Em segundo lugar, a implementação do programa delineado acima requer uma teoria matemática suficientemente bem desenvolvida e completa, o que inclui:

- uma classificação da classe de grupos pertinente,

- uma classificação de suas representações irredutíveis,

- uma classificação de seus subgrupos (maximais),

- algorítmos e tabelas para regras de ramificação.

Ocorre que não existe uma teoria tão completa para a classe de todos os grupos de Lie, mas ela existe para a classe dos grupos de Lie compactos. Ademais, como consideramos apenas subgrupos fechados, nunca saímos da classe dos grupos de Lie compactos. Um outro fator importante é que qualquer representação de um grupo de Lie compacto é completamente redutível, fato este que justifica usar o conceito de regras de ramificação.

Graças ao teorema de correspondência entre grupos de Lie conexos e álgebras de Lie, que inclusive relaciona suas representações, podemos transferir o problema inteiramente para o contexto das álgebras de Lie. Notemos que aos grupos de Lie compactos conexos correspondem as álgebras de Lie compactas e que o problema de como tratar os subgrupos de Lie não fechados desaparece quando passamos às álgebras de Lie. Portanto, fixando uma determinada distribuição de multipletos com soma total de dimensões igual a $n$, a construção de um modelo algébrico baseado no princípio de simetrias quebradas pode, no contexto dos grupos de Lie compactos conexos, ser implementada pela seguinte estratégia:

a) Determinar todas as álgebras de Lie compactas simples que possuem representações irredutíveis de dimensão $n$;

b) Para cada representação irredutível encontrada analisar as regras de ramificação da representação em relação a todas as cadeias descendentes de subalgebras maximais.

Observemos também que, como vimos no capítulo anterior, toda álgebra de Lie compacta é redutiva, isto é, decompõe-se na soma direta de seu centro e uma subalgebra semisimples. Suponha que $\mathfrak{g}$ é uma álgebra de Lie semisimples compacta e $\mathfrak{h}$ uma subalgebra maximal de $\mathfrak{g}$, então $\mathfrak{h}$ é necessáriamente redutiva e podemos escrever

$$
\mathfrak{h}=\mathfrak{z}(\mathfrak{h}) \oplus \mathfrak{h}_{1} \oplus \ldots \oplus \mathfrak{h}_{k},
$$


Quebra de Simetrias no Código Genético

onde $\mathfrak{z}(\mathfrak{h})$ é o centro (e portanto um ideal abeliano) de $\mathfrak{h}$ e $\mathfrak{h}_{1}, \ldots, \mathfrak{h}_{k}$ são os ideais simples de $\mathfrak{h}$. Considere agora uma representação irredutível de $\mathfrak{g}$ em um espaço vetorial $V$. Quando restringirmos esta representação à subalgebra h obtemos uma representação completamente redutível de $\mathfrak{h}$, pois esta é a álgebra de Lie de um grupo compacto. Denotemos por $V_{i}$ $(i=1, \ldots, \ell)$ os subespaços invariantes sob h. Então cada subespaço $V_{i}$ carrega uma representação irredutível de $\mathfrak{h}$ que se decompõe em um produto tensorial de representações irredutíveis de cada um dos ideais $\mathfrak{z}(\mathfrak{h}), \mathfrak{h}_{1}, \ldots, \mathfrak{h}_{k}$ de $\mathfrak{h}$. Mas, como toda representação irredutível de $\mathfrak{z}(\mathfrak{h})$ é unidimensional, a dimensão de $V_{i}$ só depende das representações dos ideais não-abelianos de h. Assim concluímos que não há perda de generalidade ao nos restringirmos a cadeias de subalgebras semisimples maximais, pois estamos interessados apenas nas dimensões e nas multiplicidades dos multipletos. e podemos substituir o item b) acima por

c) Para cada representação irredutível encontrada analisar as regras de ramificação da representação em relação a todas as cadeias descendentes de subalgebras semisimples maximais.

Uma resposta parcial ao item a) pode ser encontrada em [35], onde estão tabeladas as representações irredutíveis de dimensão $\leqslant 60.000 .000$ das álgebras de Lie simples de posto $\leqslant 8$. Para responder ao item c), precisamos recorrer ao teorema de estrutura das subalgebras maximais das álgebras de Lie semisimples que demonstramos no capítulo anterior.

\subsection{Produto Tensorial e Restrição de Representações}

Dada uma representação $\rho$ de uma álgebra de Lie semisimples $\mathfrak{g}$ em um espaço vetorial $V$ queremos determinar a regra de ramificação da restrição de $\rho$ a uma subalgebra maximal $\widetilde{\mathfrak{g}}$ de $\mathfrak{g}$. Como $\mathfrak{g}$ é semisimples podemos decompo-la em soma direta de ideais simples

$$
\mathfrak{g}=\mathfrak{g}_{1} \oplus \ldots \oplus \mathfrak{g}_{k} .
$$

Pelo teorema 1.2.1 da seção 1.2 há duas possibilidades (a menos de isomorfismos) para a subalgebra $\tilde{\mathfrak{g}}$ :

- tipo simples: escrevendo $\mathfrak{g}=\mathfrak{g}^{\prime} \oplus \mathfrak{g}_{i}$, onde $\mathfrak{g}_{i}(1 \leqslant i \leqslant k)$ é um ideal simples de $\mathfrak{g}$ e $\mathfrak{g}^{\prime}$ é o ideal complementar a $\mathfrak{g}_{i}$ (isto é, a soma direta de todos os outros ideais simples de $\mathfrak{g})$, temos que $\widetilde{\mathfrak{g}}=\mathfrak{g}^{\prime} \oplus \widetilde{\mathfrak{g}_{i}}$ onde $\widetilde{\mathfrak{g}_{i}}$ é uma subalgebra maximal de $\mathfrak{g}_{i}$.

- tipo diagonal: escrevendo $\mathfrak{g}=\mathfrak{g}^{\prime} \oplus \mathfrak{g}_{0} \oplus \mathfrak{g}_{0}$, onde $\mathfrak{g}_{0}$ é um ideal de $\mathfrak{g}$ que aparece (pelo menos) duas vezes na decomposição $(2.2)$ e $\mathfrak{g}^{\prime}$ é o ideal complementar a $\mathfrak{g}_{0} \oplus \mathfrak{g}_{0}$ (isto é, a soma direta de todos os outros ideais simples de $\mathfrak{g}$ ), temos que $\widetilde{\mathfrak{g}}=\mathfrak{g}^{\prime} \oplus \widetilde{\mathfrak{g}_{0}}$ onde $\widetilde{\mathfrak{g}_{0}}$ é a inclusão diagonal de $\mathfrak{g}_{0}$ em $\mathfrak{g}_{0} \oplus \mathfrak{g}_{0}\left(X \in \mathfrak{g}_{0}\right.$ é levado em $\left.(X, X) \in \mathfrak{g}_{0} \oplus \mathfrak{g}_{0}\right)$. 
Então estudar a restrição de $\rho$ a uma destas subalgebras é equivalente a estudar os seguintes problemas:

1. Dada uma álgebra de Lie simples $\mathfrak{g}$ e uma representação irredutível $\rho$ de $\mathfrak{g}$ determinar a regra de ramificação de $\operatorname{Res}_{\mathfrak{f}}^{\mathfrak{g}}(\rho)$ para toda subalgebra maximal $\mathfrak{f}$ de $\mathfrak{g}$.

2. Dada uma álgebra de Lie simples $\mathfrak{f}$ e uma representação irredutível $\rho$ de $\mathfrak{g}=\mathfrak{f} \times \mathfrak{f}$ determinar a regra de ramificação de $\operatorname{Res}_{\mathfrak{f}}^{\mathfrak{g}}(\rho)$ onde $\mathfrak{f}$ é a inclusão diagonal de $\mathfrak{f}$ em $\mathfrak{g}=\mathfrak{f} \times \mathfrak{f}:$

$$
\begin{aligned}
\mathfrak{f} & \longrightarrow \mathfrak{f} \times \mathfrak{f} \\
X & \longmapsto(X, X)
\end{aligned}
$$

O problema 1 é muito importante em física e por este motivo há muita pesquisa nesta direção, resultando numa variedade enorme de fórmulas que o resolvem em casos particulares (para certas subalgebras de certas álgebras de Lie simples). Algumas destas fórmulas podem ser encontradas em [15, pag. 424] e [61]. Há um tratamento mais uniforme dado por McKay \& Patera [35] que determinaram todas as regras de ramificação para as álgebras simples com posto menor ou igual a 8 e construíram tabelas que permitem uma consulta rápida e direta. Esta tabelas foram construídas a partir da classificação de Dynkin que apresentamos no capítulo 1, e portanto contêm as regras de ramificação para todas as subalgebras redutivas maximais das álgebras simples. O método utilizado para o cálculo destas regras de ramificação está exposto em [40] e [36], sendo que nesta última referência é apresentada uma listagem do programa que gerou as tabelas de [35].

Consideremos agora o problema 2. Neste caso $\rho$ é o produto tensorial de duas representações irredutíveis $\rho_{1}$ e $\rho_{2}$ de $\mathfrak{f}$ em $V_{1}$ e $V_{2}$, respectivamente. Então a restrição de $\rho$ à inclusão diagonal de $\mathfrak{f}$ em $\mathfrak{f} \times \mathfrak{f}$ é simplesmente a representação $\rho_{1} \otimes \rho_{2}$ de $\mathfrak{f}$ em $V_{1} \otimes V_{2}$. De fato, como

$$
\rho(X, Y)\left(v_{1} \otimes v_{2}\right)=\rho_{1}(X) v_{1} \otimes v_{2}+v_{1} \otimes \rho_{2}(Y) v_{2}
$$

para todo $(X, Y) \in \mathfrak{g}, v_{1} \in V_{1}$ e $v_{2} \in V_{2}$, a restrição de $\rho$ a $\mathfrak{f}$ é dada por

$$
\operatorname{Res}_{\mathfrak{f}}^{\mathfrak{g}}(\rho)(X, X)\left(v_{1} \otimes v_{2}\right)=\rho_{1}(X) v_{1} \otimes v_{2}+v_{1} \otimes \rho_{2}(X) v_{2}=\left(\left(\rho_{1} \otimes \rho_{2}\right)(X)\right)\left(v_{1} \otimes v_{2}\right)
$$

para todo $(X, X) \in \mathfrak{f}, v_{1} \in V_{1}$ e $v_{2} \in V_{2}$. Sendo esta representação completamente redutível podemos escrever

$$
\rho_{1} \otimes \rho_{2}=\bigoplus_{i=1}^{k} \rho_{i} \quad, \quad V_{1} \otimes V_{2}=\bigoplus_{i=1}^{k} W_{i}
$$

onde $\rho_{i}$ são representações irredutíveis de $\mathfrak{f}$ em espaços $W_{i}(i=1, \ldots, k)$. Ou seja, enfrentamos o problema clássico de decompor um produto tensorial de duas representações irredutíveis de uma álgebra de Lie simples em soma direta de representações irredutíveis, também 
conhecido como problema de "Clebsch-Gordan". Este problema já é estudado há muito tempo por matemáticos e físicos, e existem várias fórmulas para vários tipos de álgebras de Lie simples. A mais conhecida delas é a clássica fórmula de Clebsch-Gordan, muito importante na teoria do momento angular em mecânica quântica [49], que fornece a decomposição do produto tensorial de duas representações irredutíveis da álgebra de Lie simples $A_{1}$. Seja $\rho_{s}$ a representação de spin $s$ de $A_{1}$, então

$$
\rho_{s_{1}} \otimes \rho_{s_{2}}=\rho_{\left|s_{1}-s_{2}\right|} \oplus \rho_{\left|s_{1}-s_{2}\right|+1} \oplus \ldots \oplus \rho_{\left|s_{1}+s_{2}\right|-1} \oplus \rho_{\left|s_{1}+s_{2}\right|} .
$$

Usando a notação que introduzimos no capítulo 1 e lembrando que o peso máximo da representação $V_{s}$ é igual a $2 s$, podemos escrever a fórmula (2.3) em termos de pesos máximos

$$
(n) \otimes(m)=(|m-n|) \oplus(|m-n+2|) \oplus \ldots \oplus(m+n) .
$$

Por exemplo,

$$
\begin{aligned}
& (1) \otimes(1)=(0) \oplus(1), \\
& (1) \otimes(2)=(1) \oplus(3), \\
& (2) \otimes(2)=(0) \oplus(2) \oplus(4) .
\end{aligned}
$$

Em geral, podemos formular o problema nos seguintes termos: Dadas duas representações irredutíveis $\Gamma_{\lambda}$ e $\Gamma_{\mu}$ de uma álgebra de Lie semisimples com pesos máximos $\lambda$ e $\mu$, introduzimos inteiros não-negativos $N_{\lambda \mu}^{\nu}$ tais que

$$
\Gamma_{\lambda} \otimes \Gamma_{\mu}=\bigoplus_{\nu} N_{\lambda \mu}^{\nu} \Gamma_{\nu}
$$

Os números $N_{\lambda \mu}^{\nu}$ são chamados multiplicidades externas ou coeficientes de fusão da decomposição (2.5). Existem fórmulas combinatórias que descrevem os coeficientes $N_{\lambda \mu}^{\nu}$. A mais conhecida é a chamada regra de Littlewood-Richardson para as álgebras do tipo $A_{n}$ (ver [15]), e em [58] há algumas fórmulas combinatórias para as outras álgebras clássicas. Existe também uma fórmula geral devida a Steinberg [20, pag. 141] que fornece os coeficientes $N_{\lambda \mu}^{\nu}$ para uma álgebra de Lie $\mathfrak{g}$ semisimples qualquer:

$$
N_{\lambda \mu}^{\nu}=\sum_{w_{1}, w_{2} \in \mathcal{W}} \operatorname{sgn}\left(w_{1} w_{2}\right) \mathcal{P}\left(\nu+2 \varrho-w_{1}(\lambda+\varrho)-w_{2}(\mu+\varrho)\right)
$$

onde $\mathcal{W}$ é o grupo de Weyl de $\mathfrak{g}$, $\mathcal{P}$ é a função de Kostant definida como sendo o número de subconjuntos de inteiros não-negativos $\left\{n_{\alpha} \mid \alpha \in \Delta^{+}\right\}$para os quais

$$
\lambda=-\sum_{\alpha \in \Delta^{+}} n_{\alpha} \alpha
$$


e

$$
\varrho=\frac{1}{2} \sum_{\alpha \in \Delta^{+}} \alpha .
$$

onde $\Delta^{+}$é o conjunto de raizes positivas de $\mathfrak{g}$. Esta fórmula é bem explícita, porém não é fácil de aplicar quando o grupo de Weyl é muito grande.

\subsection{Subalgebras Maximais das Álgebras Simples para o Código Genético}

Agora que já temos uma teoria para implementar o modelo geral de quebra de simetrias podemos analisar o caso particular do código genético. Uma apresentação detalhada da estrutura do código genético se encontra em [23]. Este trabalho contém também alguns argumentos físicos e biológicos para a utilização de modelos baseados no princípio de quebra de simetrias.

No caso do código genético a distribuição de multipletos é a seguinte:

- 2 singletos,

- 9 dubletos,

- 2 tripletos,

- 5 quartetos,

- 3 sextetos.

Temos portanto 21 multipletos cujas dimensões somam 64 .

Precisamos então determinar todas as álgebras de Lie compactas simples que possuem representações irredutíveis de dimensão $64^{1}$. Este problema foi resolvido em [23] e as álgebras compactas simples com os pesos máximos das representações irredutíveis de dimensão 64, chamadas representações de códons, estão apresentadas na tabela 2.1. Também calculamos o tipo de cada uma, de acordo com o teorema 1.4.3 do capítulo 1. Observe que nesta tabela as 8 primeiras álgebras possuem posto menor que 8 e portanto constam das tabelas de McKay \& Patera [35]. As três últimas são álgebras de matrizes naturalmente definidas em espaços vetoriais de qualquer dimensão e portanto suas representações de códons são suas representações fundamentais. O fato de que não há outras representações de códons é não-trivial e decorre de uma análise baseada na fórmula de dimensão de Weyl. Um fato

\footnotetext{
${ }^{1} \mathrm{~A}$ partir de agora só consideraremos álgebras de Lie compactas.
} 
interessante é que esta tabela apresenta todas as subalgebras simples irredutíveis de $\mathfrak{s u}(64)$, e de acordo com o teorema de Dynkin (ver tabela 1.6) todas são maximais em $\mathfrak{s u}(64), \mathfrak{s o}(64)$ ou $\mathfrak{s p}(64)$, de acordo com o tipo que aparece na última coluna. Esta observação deixa claro, através de um exemplo não-trivial, que a parte mais difícil em relação à utilização do referido teorema de Dynkin é a determinação das álgebras que possuem representações irredutíveis de uma determinada dimensão.

\begin{tabular}{|c|c|c|c|}
\hline $\begin{array}{c}\text { Notação } \\
\text { de Cartan }\end{array}$ & $\begin{array}{c}\text { Álgebra de Lie } \\
\text { Compacta Simples }\end{array}$ & $\begin{array}{c}\text { Peso } \\
\text { Máximo }\end{array}$ & Tipo \\
\hline \hline$A_{1}$ & $\mathfrak{s u}(2)$ & 63 & -1 \\
$A_{2}$ & $\mathfrak{s u}(3)$ & $(33)$ & 1 \\
$B_{2}$ & $\mathfrak{s o}(5)$ & $(13)$ & -1 \\
$G_{2}$ & $(11)$ & 1 \\
$A_{3}$ & $\mathfrak{s u}(4)$ & $(111)$ & 1 \\
$C_{3}$ & $\mathfrak{s p}(6)$ & $(110)$ & -1 \\
$B_{6}$ & $\mathfrak{s o}(13)$ & $(000001)$ & -1 \\
$D_{7}$ & $\mathfrak{s o}(14)$ & $(0000010)$ & 0 \\
$C_{32}$ & $\mathfrak{s p}(64)$ & $(10 \ldots 0)$ & 0 \\
$D_{32}$ & $\mathfrak{s o}(64)$ & $(10 \ldots 0)$ & 0 \\
$A_{63}$ & $\mathfrak{s u}(64)$ & $(10 \ldots 0)$ & 0 \\
\hline
\end{tabular}

Tabela 2.1: Representações de Códons.

Agora podemos usar as tabelas do capítulo anterior para determinar todas as subalgebras maximais das álgebras de Lie simples que aparecem em alguma cadeia proveniente de uma das álgebras da parte superior da tabela 2.1. Na verdade precisaremos das subalgebras maximais de quase todas as álgebras clássicas de posto $\leqslant 7$, mais as subalgebras maximais da álgebra excepcional $G_{2}$. Estas já se encontram na tabela 1.7. Para as demais precisaremos 
consultar as tabelas 1.4, 1.5 e 1.6 .

Os resultados serão apresentados em forma de tabelas, que possuem três colunas: a primeira apresenta as subalgebras do modo em que são fornecidas pelas tabelas do capítulo anterior e a segunda na notação de Cartan, já levando em conta os isomorfismos

$$
C_{1}=B_{1}=A_{1}, \quad C_{2}=B_{2}, \quad D_{2}=A_{1} \oplus A_{1}, \quad D_{3}=A_{3},
$$

(adotaremos a convenção usual em que $A_{n}(n \geqslant 1), B_{n}(n \geqslant 2), C_{n}(n \geqslant 3), D_{n}(n \geqslant 4)$ e portanto na segunda coluna não aparecem $B_{1}, C_{1}, C_{2}, D_{1}, D_{2}, D_{3}\left(D_{1}\right.$ seria $\mathfrak{s o}(2)$ que não é simples)), e finalmente a última coluna indica o tipo da subalgebra de acordo com a caracterização explicada no capítulo anterior:

- tipo redutível, indicado pela letra $\mathcal{R}$, seguida pelo número que representa o tipo na tabela 1.4;

- tipo irredutível não-simples, indicado pela letra $\mathcal{N}$, seguida pelo número que representa o tipo na tabela 1.5;

- tipo irredutível simples, indicado pela letra $\mathcal{S}$, seguida pelo peso máximo da representação da subalgebra que define a inclusão, quando não se tratar de um espaço simétrico.

Além disto indicaremos nesta última coluna o espaço simétrico associado à subalgebra, quando for o caso, colocando o tipo de acordo com a notaçõa padrão utilizada em [19].

Como só estamos interessados nas subalgebras semisimples maximais, nossas tabelas não contêm subalgebras que possuem centro não-trivial e são maximais, de acordo com as tabelas do capítulo anterior, quando sua parte semisimples não é maximal. A tabela 1.4 é a única que possui subalgebras com centro não-trivial e logo abaixo desta tabela já haviamos indicado quais destas deixam de ser maximais após remoção do centro: este fenômeno ocorre apenas com as álgebras ortogonais $\left(B_{n}\right.$ e $\left.D_{n}\right)$ e em alguns casos isolados que serão comentados no item 3 abaixo.

Como já foi mencionado, a parte mais difícil da tarefa é classificar as subalgebras irredutíveis simples, o que requer determinar todas as álgebras simples que possuem representações irredutíveis de uma dimensão fixada. As seguintes observações facilitam o procedimento.

1. Como a álgebra $\mathfrak{s u}(2)$ admite uma representação irredutível em cada dimensão e esta representação é sempre auto-dual existe uma subalgebra maximal irredutível $\mathfrak{s u}(2)$ em $\mathfrak{s o}(n)$ quando $n$ for impar (o peso máximo de uma representação irredutível de $\mathfrak{s u}(2)$ de dimensão impar é um número par e portanto a representação é ortogonal) e uma 
subalgebra maximal irredutível $\mathfrak{s u}(2)$ em $\mathfrak{s p}(n)$ quando $n$ for par (o peso máximo de uma representação irredutível de $\mathfrak{s u}(2)$ de dimensão par é um número impar e portanto a representação é simplética). A única exceção é a álgebra $\mathfrak{s o ( 7 ) , ~ n a ~ q u a l ~ a ~ s u b a l g e b r a ~}$ irredutível $\mathfrak{s u}(2)$ não é maximal, segundo a tabela 1.6.

2. Toda álgebra $\mathfrak{s u}(n)$ possui subalgebras maximais irredutíveis naturais: $\mathfrak{s o}(n)$ quando $n$ for impar e $\mathfrak{s o}(n)$ e $\mathfrak{s p}(n)$ quando $n$ for par. Estas inclusões são determinadas pela representação fundamental e definem os espaços simétricos de tipo $A I$ no caso de $\mathfrak{s o}(n)$ e $A I I$ no caso de $\mathfrak{s} \mathfrak{p}(n)$. Nestes dois casos não indicaremos o peso máximo das representações.

3. Há três casos excepcionais de subalgebras que, contrariando as expectativas, não aparecem nas tabelas que seguem:

(a) $\mathfrak{s u}(4) \supset \mathfrak{s o}(4)$, do tipo $(\mathcal{S} ; A I)$ : devido ao fato de $\mathfrak{s o}(4) \cong \mathfrak{s u}(2) \oplus \mathfrak{s u}(2)$ não ser simples, ela é de fato uma subalgebra maximal irredutível não-simples (tipo $\mathcal{N}$, não tipo $\mathcal{S})$;

(b) $\mathfrak{s u}(4) \supset \mathbb{R} \oplus \mathfrak{s u}(2) \oplus \mathfrak{s u}(2)$, do tipo (RI; $A$ III): devido ao fato de que $\mathfrak{s u}(2) \oplus \mathfrak{s u}(2) \cong \mathfrak{s o}(4)$, a parte semisimples desta subalgebra não é maximal, pois

$$
\mathfrak{s u}(2) \oplus \mathfrak{s u}(2) \cong \mathfrak{s o}(4) \subset \mathfrak{s o}(5) \subset \mathfrak{s o}(6) \cong \mathfrak{s u}(4) .
$$

(c) $\mathfrak{s o}(8) \supset \mathfrak{u}(4)$, do tipo $(\mathcal{R} ; D I I I)$ : devido ao fato de que $\mathfrak{s u}(4) \cong \mathfrak{s o}(6)$, a parte semisimples desta subalgebra não é maximal, pois

$$
\mathfrak{s u}(4) \cong \mathfrak{s o}(6) \subset \mathfrak{s o}(7) \supset \mathfrak{s o}(8) .
$$

Devemos mencionar também que as álgebras $\mathfrak{s u}(6)$ e $\mathfrak{s o}(8)$ contêm subalgebras maximais irredutíveis simples que não são conjugadas mas são levadas umas nas outras por automorfismos externos. No caso de $\mathfrak{s u}(6)$, existem duas classes de conjugação de subalgebras $\mathfrak{s u}(3)$ dadas pelos pesos máximos (20) e (02) e relacionadas por conjugação complexa, que é um automorfismo externo de $\mathfrak{s u}(6)$. No caso de $\mathfrak{s o}(8)$, cujo grupo de automorfismos externos é o grupo simétrico $S_{3}$, existem duas classes de conjugação de subalgebras $\mathfrak{s o}(7)$ e duas classes de conjugação de subalgebras do tipo $A_{1} \oplus B_{2}$ relacionadas pelo automorfismo externo de $\mathfrak{s o}(8)$ que transforma a representação vetorial em uma das duas spinoriais. Nas tabelas de McKay \& Patera [35], apenas uma de cada uma dessas duas classes é listada, o que deve ser devidamente levado em consideração quando se calculam regras de ramificação. 


\begin{tabular}{|c|c|c|}
\hline $\begin{array}{c}\text { Subalgebra } \\
\text { Maximal }\end{array}$ & $\begin{array}{c}\text { Notação } \\
\text { de Cartan }\end{array}$ & Classe \\
\hline \hline $\mathfrak{u}(2)$ & $A_{1}$ & $(\mathcal{R I} ; A I I I)$ \\
\hline $\mathfrak{s o}(3)$ & $\tilde{A}_{1}$ & $(\mathcal{S} ; A I)$ \\
\hline
\end{tabular}

Tabela 2.2: Subalgebras maximais de $A_{2}=\mathfrak{s u}(3)$.

\begin{tabular}{|c|c|c|}
\hline $\begin{array}{c}\text { Subalgebra } \\
\text { Maximal }\end{array}$ & $\begin{array}{c}\text { Notação } \\
\text { de Cartan }\end{array}$ & Classe \\
\hline \hline $\mathfrak{u}(3)$ & $A_{2}$ & $(\mathcal{R I} ; A I I I)$ \\
\hline $\mathfrak{s u}(2) \times \mathfrak{s u}(2)$ & $A_{1} \oplus A_{1}$ & $(\mathcal{N I})$ \\
\hline $\mathfrak{s p}(4)$ & $B_{2}$ & $(\mathcal{S} ; A I I)$ \\
\hline
\end{tabular}

Tabela 2.3: Subalgebras maximais de $A_{3}=\mathfrak{s u}(4)$.

\begin{tabular}{|c|c|c|}
\hline $\begin{array}{c}\text { Subalgebra } \\
\text { Maximal }\end{array}$ & $\begin{array}{c}\text { Notação } \\
\text { de Cartan }\end{array}$ & Classe \\
\hline \hline $\mathfrak{u}(4)$ & $A_{3}$ & $(\mathcal{R I} ; A I I I)$ \\
\hline $\mathbb{R} \oplus \mathfrak{s u}(2) \oplus \mathfrak{s u}(3)$ & $A_{1} \oplus A_{2}$ & $(\mathcal{R I} ; A I I I)$ \\
\hline $\mathfrak{s o}(5)$ & $B_{2}$ & $(\mathcal{S} ; A I)$ \\
\hline
\end{tabular}

Tabela 2.4: Subalgebras maximais de $A_{4}=\mathfrak{s u}(5)$. 


\begin{tabular}{|c|c|c|}
\hline $\begin{array}{c}\text { Subalgebra } \\
\text { Maximal }\end{array}$ & $\begin{array}{c}\text { Notação } \\
\text { de Cartan }\end{array}$ & Classe \\
\hline \hline $\mathfrak{u}(5)$ & $A_{4}$ & $(\mathcal{R I} ; A I I I)$ \\
\hline $\mathbb{R} \oplus \mathfrak{s u}(2) \oplus \mathfrak{s u}(4)$ & $A_{1} \oplus A_{3}$ & $(\mathcal{R I} ; A I I I)$ \\
\hline $\mathbb{R} \oplus \mathfrak{s u}(3) \oplus \mathfrak{s u}(3)$ & $A_{2} \oplus A_{2}$ & $(\mathcal{R I} ; A I I I)$ \\
\hline $\mathfrak{s u}(2) \times \mathfrak{s u}(3)$ & $A_{1} \oplus A_{2}$ & $(\mathcal{N I})$ \\
\hline $\mathfrak{s u}(3)$ & $A_{2}$ & $(\mathcal{S} ;(20))$ \\
\hline $\mathfrak{s u}(3)$ & $A_{2}$ & $(\mathcal{S} ;(02))$ \\
\hline $\mathfrak{s o}(6)$ & $A_{3}$ & $(\mathcal{S} ; A I)$ \\
\hline $\mathfrak{s p}(6)$ & $C_{3}$ & $(\mathcal{S} ; A I I)$ \\
\hline
\end{tabular}

Tabela 2.5: Subalgebras maximais de $A_{5}=\mathfrak{s u}(6)$.

\begin{tabular}{|c|c|c|}
\hline $\begin{array}{c}\text { Subalgebra } \\
\text { Maximal }\end{array}$ & $\begin{array}{c}\text { Notação } \\
\text { de Cartan }\end{array}$ & Classe \\
\hline \hline $\mathfrak{u}(6)$ & $A_{5}$ & $(\mathcal{R I} ; A I I I)$ \\
\hline $\mathbb{R} \oplus \mathfrak{s u}(2) \oplus \mathfrak{s u}(5)$ & $A_{1} \oplus A_{4}$ & $(\mathcal{R I} ; A I I I)$ \\
\hline $\mathbb{R} \oplus \mathfrak{s u}(3) \oplus \mathfrak{s u}(4)$ & $A_{2} \oplus A_{3}$ & $(\mathcal{R I} ; A I I I)$ \\
\hline $\mathfrak{s o}(7)$ & $B_{3}$ & $(\mathcal{S} ; A I)$ \\
\hline
\end{tabular}

Tabela 2.6: Subalgebras maximais de $A_{6}=\mathfrak{s u}(7)$. 


\begin{tabular}{|c|c|c|}
\hline $\begin{array}{c}\text { Subalgebra } \\
\text { Maximal }\end{array}$ & $\begin{array}{c}\text { Notação } \\
\text { de Cartan }\end{array}$ & Classe \\
\hline \hline $\mathfrak{s o}(4)$ & $A_{1} \oplus A_{1}$ & $(\mathcal{R I I} ; B D I)$ \\
\hline $\mathfrak{s u}(2)$ & $A_{1}$ & $(\mathcal{S} ; 4)$ \\
\hline
\end{tabular}

Tabela 2.7: Subalgebras maximais de $B_{2}=\mathfrak{s o}(5)$.

\begin{tabular}{|c|c|c|}
\hline $\begin{array}{c}\text { Subalgebra } \\
\text { Maximal }\end{array}$ & $\begin{array}{c}\text { Notação } \\
\text { de Cartan }\end{array}$ & Classe \\
\hline \hline $\mathfrak{s o}(6)$ & $A_{3}$ & $(\mathcal{R I I} ; B D I)$ \\
\hline $\mathfrak{s o}(3) \oplus \mathfrak{s o}(4)$ & $A_{1} \oplus A_{1} \oplus A_{1}$ & $(\mathcal{R I I} ; B D I)$ \\
\hline$G_{2}$ & $G_{2}$ & $(\mathcal{S} ;(01))$ \\
\hline
\end{tabular}

Tabela 2.8: Subalgebras maximais de $B_{3}=\mathfrak{s o}(7)$.

\begin{tabular}{|c|c|c|}
\hline $\begin{array}{c}\text { Subalgebra } \\
\text { Maximal }\end{array}$ & $\begin{array}{c}\text { Notação } \\
\text { de Cartan }\end{array}$ & Classe \\
\hline \hline $\mathfrak{s o}(8)$ & $D_{4}$ & $(\mathcal{R I I} ; B D I)$ \\
\hline $\mathfrak{s o}(3) \oplus \mathfrak{s o}(6)$ & $A_{1} \oplus A_{3}$ & $(\mathcal{R I I} ; B D I)$ \\
\hline $\mathfrak{s o}(4) \oplus \mathfrak{s o}(5)$ & $A_{1} \oplus A_{1} \oplus B_{2}$ & $(\mathcal{R I I} ; B D I)$ \\
\hline $\mathfrak{s o}(3) \times \mathfrak{s o}(3)$ & $A_{1} \oplus A_{1}$ & $(\mathcal{N I I})$ \\
\hline $\mathfrak{s u}(2)$ & $A_{1}$ & $(\mathcal{S} ; 8)$ \\
\hline
\end{tabular}

Tabela 2.9: Subalgebras maximais de $B_{4}=\mathfrak{s o}(9)$. 


\begin{tabular}{|c|c|c|}
\hline $\begin{array}{c}\text { Subalgebra } \\
\text { Maximal }\end{array}$ & $\begin{array}{c}\text { Notação } \\
\text { de Cartan }\end{array}$ & Classe \\
\hline \hline $\mathfrak{s o}(10)$ & $D_{5}$ & $(\mathcal{R I I} ; B D I)$ \\
\hline $\mathfrak{s o}(3) \oplus \mathfrak{s o}(8)$ & $A_{1} \oplus D_{4}$ & $(\mathcal{R I I} ; B D I)$ \\
\hline $\mathfrak{s o}(4) \oplus \mathfrak{s o}(7)$ & $A_{1} \oplus A_{1} \oplus B_{3}$ & $(\mathcal{R I I} ; B D I)$ \\
\hline $\mathfrak{s o}(5) \oplus \mathfrak{s o}(6)$ & $B_{2} \oplus A_{3}$ & $(\mathcal{R I I} ; B D I)$ \\
\hline $\mathfrak{s u}(2)$ & $A_{1}$ & $(\mathcal{S} ; 10)$ \\
\hline
\end{tabular}

Tabela 2.10: Subalgebras maximais de $B_{5}=\mathfrak{s o}(11)$.

\begin{tabular}{|c|c|c|}
\hline $\begin{array}{c}\text { Subalgebra } \\
\text { Maximal }\end{array}$ & $\begin{array}{c}\text { Notação } \\
\text { de Cartan }\end{array}$ & Classe \\
\hline \hline $\mathfrak{s o}(12)$ & $D_{6}$ & $(\mathcal{R I I} ; B D I)$ \\
\hline $\mathfrak{s o}(3) \oplus \mathfrak{s o}(10)$ & $A_{1} \oplus D_{5}$ & $(\mathcal{R I I} ; B D I)$ \\
\hline $\mathfrak{s o}(4) \oplus \mathfrak{s o}(9)$ & $A_{1} \oplus A_{1} \oplus B_{4}$ & $(\mathcal{R I I} ; B D I)$ \\
\hline $\mathfrak{s o}(5) \oplus \mathfrak{s o}(8)$ & $B_{2} \oplus D_{4}$ & $(\mathcal{R I I} ; B D I)$ \\
\hline $\mathfrak{s o}(6) \oplus \mathfrak{s o}(7)$ & $A_{3} \oplus B_{3}$ & $(\mathcal{R I I} ; B D I)$ \\
\hline $\mathfrak{s u}(2)$ & $A_{1}$ & $(\mathcal{S} ; 12)$ \\
\hline
\end{tabular}

Tabela 2.11: Subalgebras maximais de $B_{6}=\mathfrak{s o}(13)$. 


\begin{tabular}{|c|c|c|}
\hline $\begin{array}{c}\text { Subalgebra } \\
\text { Maximal }\end{array}$ & $\begin{array}{c}\text { Notação } \\
\text { de Cartan }\end{array}$ & Classe \\
\hline \hline $\mathfrak{s p}(2) \oplus \mathfrak{s p}(4)$ & $A_{1} \oplus B_{2}$ & $(\mathcal{R I I I} ; C I I)$ \\
\hline $\mathfrak{u}(3)$ & $A_{2}$ & $(\mathcal{R I V} ; C I)$ \\
\hline $\mathfrak{s p}(2) \times \mathfrak{s o}(3)$ & $A_{1} \oplus A_{1}$ & $(\mathcal{N I V})$ \\
\hline $\mathfrak{s u}(2)$ & $A_{1}$ & $(\mathcal{S} ; 5)$ \\
\hline
\end{tabular}

Tabela 2.12: Subalgebras maximais de $C_{3}=\mathfrak{s p}(6)$. 


\begin{tabular}{|c|c|c|}
\hline $\begin{array}{c}\text { Subalgebra } \\
\text { Maximal }\end{array}$ & $\begin{array}{c}\text { Notação } \\
\text { de Cartan }\end{array}$ & Classe \\
\hline \hline $\mathfrak{s o}(7)$ & $B_{3}$ & $(\mathcal{R V I} ; B D I)$ \\
\hline $\mathfrak{s o}(3) \oplus \mathfrak{s o}(5)$ & $A_{1} \oplus B_{2}$ & $(\mathcal{R V I} ; B D I)$ \\
\hline $\mathfrak{s o}(4) \oplus \mathfrak{s o}(4)$ & $A_{1} \oplus A_{1} \oplus A_{1} \oplus A_{1}$ & $(\mathcal{R V} ; B D I)$ \\
\hline $\mathfrak{s p}(2) \times \mathfrak{s p}(4)$ & $A_{1} \oplus B_{2}$ & $(\mathcal{N I I I})$ \\
\hline $\mathfrak{s o}(7)$ & $B_{3}$ & $(\mathcal{S} ;(001))$ \\
\hline $\mathfrak{s u}(3)$ & $A_{2}$ & $(\mathcal{S} ;(11))$ \\
\hline
\end{tabular}

Tabela 2.13: Subalgebras maximais de $D_{4}=\mathfrak{s o}(8)$.

\begin{tabular}{|c|c|c|}
\hline $\begin{array}{c}\text { Subalgebra } \\
\text { Maximal }\end{array}$ & $\begin{array}{c}\text { Notação } \\
\text { de Cartan }\end{array}$ & Classe \\
\hline \hline $\mathfrak{s o}(9)$ & $B_{4}$ & $(\mathcal{R V I} ; B D I)$ \\
\hline $\mathfrak{s o}(3) \oplus \mathfrak{s o}(7)$ & $A_{1} \oplus B_{3}$ & $(\mathcal{R V I} ; B D I)$ \\
\hline $\mathfrak{s o}(4) \oplus \mathfrak{s o}(6)$ & $A_{1} \oplus A_{1} \oplus A_{3}$ & $(\mathcal{R V} ; B D I)$ \\
\hline $\mathfrak{s o}(5) \oplus \mathfrak{s o}(5)$ & $B_{2} \oplus B_{2}$ & $(\mathcal{R V I} ; B D I)$ \\
\hline $\mathfrak{u}(5)$ & $A_{4}$ & $(\mathcal{R V I I} ; D I I I)$ \\
\hline $\mathfrak{s o}(5)$ & $B_{2}$ & $(\mathcal{S} ;(02))$ \\
\hline
\end{tabular}

Tabela 2.14: Subalgebras maximais de $D_{5}=\mathfrak{s o}(10)$. 


\begin{tabular}{|c|c|c|}
\hline $\begin{array}{c}\text { Subalgebra } \\
\text { Maximal }\end{array}$ & $\begin{array}{c}\text { Notação } \\
\text { de Cartan }\end{array}$ & Classe \\
\hline \hline $\mathfrak{s o}(11)$ & $B_{5}$ & $(\mathcal{R V I} ; B D I)$ \\
\hline $\mathfrak{s o}(3) \oplus \mathfrak{s o}(9)$ & $A_{1} \oplus B_{4}$ & $(\mathcal{R V I} ; B D I)$ \\
\hline $\mathfrak{s o}(4) \oplus \mathfrak{s o}(8)$ & $A_{1} \oplus A_{1} \oplus D_{4}$ & $(\mathcal{R V} ; B D I)$ \\
\hline $\mathfrak{s o}(5) \oplus \mathfrak{s o}(7)$ & $B_{2} \oplus B_{3}$ & $(\mathcal{R V I} ; B D I)$ \\
\hline $\mathfrak{s o}(6) \oplus \mathfrak{s o}(6)$ & $A_{3} \oplus A_{3}$ & $(\mathcal{R V} ; B D I)$ \\
\hline $\mathfrak{u}(6)$ & $A_{5}$ & $(\mathcal{R V I I} ; D I I I)$ \\
\hline $\mathfrak{s p}(2) \times \mathfrak{s p}(6)$ & $A_{1} \oplus C_{3}$ & $(\mathcal{N} I I I)$ \\
\hline
\end{tabular}

Tabela 2.15: Subalgebras maximais de $D_{6}=\mathfrak{s o}(12)$.

\begin{tabular}{|c|c|c|}
\hline $\begin{array}{c}\text { Subalgebra } \\
\text { Maximal }\end{array}$ & $\begin{array}{c}\text { Notação } \\
\text { de Cartan }\end{array}$ & Classe \\
\hline \hline $\mathfrak{s o}(13)$ & $B_{6}$ & $(\mathcal{R V I} ; B D I)$ \\
\hline $\mathfrak{s o}(3) \oplus \mathfrak{s o}(11)$ & $A_{1} \oplus B_{5}$ & $(\mathcal{R V I} ; B D I)$ \\
\hline $\mathfrak{s o}(4) \oplus \mathfrak{s o}(10)$ & $A_{1} \oplus A_{1} \oplus D_{5}$ & $(\mathcal{R V} ; B D I)$ \\
\hline $\mathfrak{s o}(5) \oplus \mathfrak{s o}(9)$ & $B_{2} \oplus B_{4}$ & $(\mathcal{R V I} ; B D I)$ \\
\hline $\mathfrak{s o}(6) \oplus \mathfrak{s o}(8)$ & $A_{3} \oplus D_{4}$ & $(\mathcal{R V} ; B D I)$ \\
\hline $\mathfrak{s o}(7) \oplus \mathfrak{s o}(7)$ & $B_{3} \oplus B_{3}$ & $(\mathcal{R V I} ; B D I)$ \\
\hline $\mathfrak{u}(7)$ & $A_{6}$ & $(\mathcal{R V I I} ; D I I I)$ \\
\hline $\mathfrak{s o}(5)$ & $B_{2}$ & $(\mathcal{S} ;(20))$ \\
\hline $\mathfrak{s} \mathfrak{p}(6)$ & $C_{3}$ & $(\mathcal{S} ;(010))$ \\
\hline$G_{2}$ & $G_{2}$ & $(\mathcal{S} ;(10))$ \\
\hline
\end{tabular}

Tabela 2.16: Subalgebras maximais de $D_{7}=\mathfrak{s o}(14)$. 


\begin{tabular}{|c|c|c|}
\hline $\begin{array}{c}\text { Subalgebra } \\
\text { Maximal }\end{array}$ & $\begin{array}{c}\text { Notação } \\
\text { de Cartan }\end{array}$ & Classe \\
\hline \hline $\mathfrak{s u}(2)$ & $A_{1}$ & Singular (Irredutível) \\
\hline $\mathfrak{s u}(3)$ & $A_{2}$ & Posto Máximo \\
\hline $\mathfrak{s u}(2) \oplus \mathfrak{s u}(2)$ & $A_{1} \oplus A_{1}$ & Posto Máximo $(G)$ \\
\hline
\end{tabular}

Tabela 2.17: Subalgebras maximais de $G_{2}$.

Observe que a tabela 2.17 simplesmente reproduz a tabela 1.7. O fato da subalgebra $\mathfrak{s u}(2)$ desta tabela ser irredutível segue de um teorema de Dynkin [10, pag. 239, teo. 16.1] que apresenta todas as álgebras excepcionais que possuem subalgebras singulares que são irredutíveis com respeito à restrição de alguma representação irredutível da álgebra excepcional que a contém. No caso acima é a representação de $G_{2}$ de peso máximo (01) e dimensão 7, portanto esta subalgebra $\mathfrak{s u}(2)$ corresponde à representação irredutível de peso máximo 6 . A subalgebra da última linha fornece o espaço simétrico excepcional $G$ que está indicado na última coluna da mesma linha.

Para completar a análise, apresentamos, nas próximas três tabelas, as subalgebras semisimples maximais das álgebras simples de posto alto que aparecem na parte inferior da tabela 2.1. Examinando estas tabelas percebe-se que, para determinar todas as cadeias de subalgebras semisimples destas álgebras, seria necessário determinar todas as subalgebras semisimples maximais das álgebras simples de posto $\leqslant 64$. No entanto, observamos que a álgebra $\mathfrak{s u}(n)$ pode gerar qualquer distribuição de multipletos cujas dimensões somam $n$ : basta considerar uma subalgebra do tipo

$$
\mathfrak{s u}\left(n_{1}\right) \oplus \ldots \oplus \mathfrak{s u}\left(n_{k}\right)
$$

onde $n=n_{1}+\ldots+n_{k}, k$ é o número de multipletos desejado e $n_{i}(i=1, \ldots, k)$ é a dimensão do $i$-ésimo multipleto. A mesma construção se aplica a $\mathfrak{s o}(n)$ e a $\mathfrak{s p}(n)$ caso todos os $n_{i}$ forem pares. Neste sentido podemos dizer que as subalgebras de posto alto não fornecem modelos interessantes, ou seja, passíveis de uma interpretação biológica, pois admitem uma flexibilidade tão grande que se torna possível gerar praticamente qualquer distribuição de multipletos. 


\begin{tabular}{|c|c|c|}
\hline $\begin{array}{c}\text { Subalgebra } \\
\text { Maximal }\end{array}$ & $\begin{array}{c}\text { Notação } \\
\text { de Cartan }\end{array}$ & Classe \\
\hline \hline $\begin{array}{c}\mathfrak{s p}(2 \ell) \oplus \mathfrak{s p}(64-2 \ell) \\
(1 \leqslant \ell \leqslant 16)\end{array}$ & $\begin{array}{c}C_{\ell} \oplus C_{32-\ell} \\
(1 \leqslant \ell \leqslant 16)\end{array}$ & $(\mathcal{R I I I} ; C I I)$ \\
\hline $\mathfrak{u}(32)$ & $A_{31}$ & $(\mathcal{R I V} ; C I)$ \\
\hline $\mathfrak{s p}(2) \times \mathfrak{s o}(32)$ & $A_{1} \oplus D_{16}$ & $(\mathcal{N I V})$ \\
\hline $\mathfrak{s p}(4) \times \mathfrak{s o}(16)$ & $B_{2} \oplus D_{8}$ & $(\mathcal{N I V})$ \\
\hline $\mathfrak{s p}(8) \times \mathfrak{s o}(8)$ & $C_{4} \oplus D_{4}$ & $(\mathcal{N I V})$ \\
\hline $\mathfrak{s u}(2)$ & $A_{1}$ & $(\mathcal{S} ; 63)$ \\
\hline $\mathfrak{s o}(5)$ & $B_{2}$ & $(\mathcal{S} ;(13))$ \\
\hline $\mathfrak{s p}(6)$ & $C_{3}$ & $(\mathcal{S} ;(110))$ \\
\hline $\mathfrak{s o}(13)$ & $B_{6}$ & $(\mathcal{S} ;(000001))$ \\
\hline
\end{tabular}

Tabela 2.18: Subalgebras maximais de $C_{32}=\mathfrak{s p}(64)$. 


\begin{tabular}{|c|c|c|}
\hline $\begin{array}{c}\text { Subalgebra } \\
\text { Maximal }\end{array}$ & $\begin{array}{c}\text { Notação } \\
\text { de Cartan }\end{array}$ & Classe \\
\hline \hline $\mathfrak{s o}(63)$ & $B_{31}$ & $(\mathcal{R V I} ; B D I)$ \\
\hline $\mathfrak{s o}(\ell+2) \oplus \mathfrak{s o}(62-\ell)$ & $\begin{array}{c}B_{\ell} \oplus B_{31-\ell} \\
D_{\ell+1} \oplus D_{31-\ell} \\
(1 \leqslant \ell \leqslant 15)\end{array}$ & $(\mathcal{R V} / \mathrm{VI} ; B D I)$ \\
\hline$(1 \leqslant \ell \leqslant 30)$ & $A_{31}$ & $(\mathcal{R V I I} ; D I I I)$ \\
\hline $\mathfrak{u}(32)$ & $D_{4} \oplus D_{4}$ & $(\mathcal{N I I})$ \\
\hline $\mathfrak{s o}(8) \times \mathfrak{s o}(8)$ & $A_{1} \oplus C_{16}$ & $(\mathcal{N I I I})$ \\
\hline $\mathfrak{s p}(2) \times \mathfrak{s p}(32)$ & $B_{2} \oplus C_{8}$ & $(\mathcal{N I I I})$ \\
\hline $\mathfrak{s p}(4) \times \mathfrak{s p}(16)$ & $C_{4} \oplus C_{4}$ & $(\mathcal{N I I I})$ \\
\hline $\mathfrak{s p}(8) \times \mathfrak{s p}(8)$ & $A_{2}$ & $(\mathcal{S} ;(33))$ \\
\hline $\mathfrak{s u}(3)$ & $A_{3}$ & $(\mathcal{S} ;(111))$ \\
\hline $\mathfrak{s u}(4)$ & $G_{2}$ & $(\mathcal{S} ;(11))$ \\
\hline$G G_{2}$ & & \\
\hline
\end{tabular}

Tabela 2.19: Subalgebras maximais de $D_{32}=\mathfrak{s o}(64)$. 


\begin{tabular}{|c|c|c|}
\hline $\begin{array}{c}\text { Subalgebra } \\
\text { Maximal }\end{array}$ & $\begin{array}{c}\text { Notação } \\
\text { de Cartan }\end{array}$ & Classe \\
\hline \hline $\mathfrak{u}(63)$ & $A_{62}$ & $(\mathcal{R I} ; A I I I)$ \\
\hline $\begin{array}{c}\mathbb{R} \oplus \mathfrak{s u}(\ell+1) \oplus \mathfrak{s u}(63-\ell) \\
(1 \leqslant \ell \leqslant 31)\end{array}$ & $\begin{array}{c}A_{\ell} \oplus A_{62-\ell} \\
(1 \leqslant \ell \leqslant 31)\end{array}$ & $(\mathcal{R I} ; A I I I)$ \\
\hline $\mathfrak{s u}(2) \times \mathfrak{s u}(32)$ & $A_{1} \oplus A_{31}$ & $(\mathcal{N I})$ \\
\hline $\mathfrak{s u}(4) \times \mathfrak{s u}(16)$ & $A_{3} \oplus A_{15}$ & $(\mathcal{N I})$ \\
\hline $\mathfrak{s u}(8) \times \mathfrak{s u}(8)$ & $A_{7} \oplus A_{7}$ & $(\mathcal{N I})$ \\
\hline $\mathfrak{s o}(14)$ & $D_{7}$ & $(\mathcal{S} ;(0000010))$ \\
\hline $\mathfrak{s o}(14)$ & $D_{7}$ & $(\mathcal{S} ;(0000001))$ \\
\hline $\mathfrak{s o}(64)$ & $D_{32}$ & $(\mathcal{S} ; A I)$ \\
\hline $\mathfrak{s p}(64)$ & $C_{32}$ & $(\mathcal{S} ; A I I)$ \\
\hline
\end{tabular}

Tabela 2.20: Subalgebras maximais de $A_{63}=\mathfrak{s u}(64)$. 


\subsection{Cadeias de Subalgebras Semisimples para o Código Genético}

Com as ferramentas colocadas a disposição nas seções anteriores, passamos a analisar as cadeias descendentes de subalgebras maximais das álgebras $B_{6}$ e $D_{7}$. Para tanto, precisamos de critérios de eliminação das cadeias que são incapazes de fornecer as degenerescências do código genético. Tais critérios já estão implícitos na análise de Hornos \& Hornos [22] e foram explicitados em [3] e [23]. São critérios simples baseados na estrutura dos multipletos do código genético.

O processo de quebra através de uma dada cadeia deve ser interrompido se ela apresentar

1. mais do que 21 multipletos;

2. mais do que 2 singletos;

3. mais do que 4 multipletos com dimensão impar;

4. um número insuficiente de multipletos de dimensões $\geqslant 6$ ou $\geqslant 4$.

Isto decorre do fato que, quando se percorre uma cadeia, o número total de multipletos e o número de singletos nunca decresce. Além disto, um multipleto de dimensão impar sempre quebra em pelo menos um multipleto de dimensão impar, mais alguns outros. O último critério reflete a necessidade de haver um número suficiente de multipletos de dimensão suficientemente grande para gerar, através de quebras posteriores, os 3 sextetos e 5 quartetos do código genético.

Por outro lado, o processo de quebra através de uma dada cadeia deve prosseguir pelo menos um passo adiante se ela apresentar

1. menos do que 21 multipletos;

2. multipletos com dimensão $\geqslant 7$;

3. mais do que 3 multipletos de dimensão 6;

4. multipletos de dimensão 5 .

Uma cadeia será considerada não-sobrevivente se satisfizer, ao mesmo tempo, pelo menos um item da primeira lista e pelo menos um item da segunda lista. 
Um outro critério importante para considerar uma cadeia como não-sobrevivente é:

\section{- Pareamento Total.}

Isto significa que o processo de redução levou a uma situação na qual todas as representações irredutíveis da subalgebra considerada vêm em pares de representações duais (conjugadas complexas) ou, no caso de representações auto-duais (auto-conjugadas), com multiplicidade par: tal pareamento de representações não pode ser eliminado por quebras de simetria subsequentes, levando a distribuições de multipletos cujas multiplicidades são todas pares e impedindo que se gere os 3 sextetos, 5 quartetos e 9 dubletos do código genético.

A estratégia geral para a busca de modelos algébricos para a evolução do código genético baseados em princípios de quebra de simetria é a seguinte:

1. Escolher uma das representações de códons da tabela 2.1.

2. Submeter esta representação à quebra de simetria através de todas as cadeias de subalgebras maximais, de acordo com as tabelas da seção anterior.

3. Após cada estágio, analisar o resultado e descartar todas as cadeias que são nãosobreviventes, de acordo com os critérios estabelecidos acima, antes de passar ao próximo estágio.

Dividiremos este procedimento em três fases consecutivas, agrupando após cada passo as cadeias sobreviventes que levaram à mesma distribuição de multipletos em famílias.

Fase 1: Quebra da simetria total até as $\mathfrak{s u}(2)$-simetrias simples. Durante a primeira fase, a quebra de simetria procede através de cadeias de subalgebras semisimples maximais, realizando quebras simples e quebras diagonais caso a álgebra a ser submetida a uma quebra diagonal for diferente de $\mathfrak{s u}(2)$. Toda cadeia termina necessariamente em uma soma direta de $p$ cópias de $\mathfrak{s u}(2)$, a mais elementar das álgebras de Lie simples, onde $p$ varia de 1 até o posto da álgebra de Lie simples original.

Fase 2: Quebra das $\mathfrak{s u}(2)$-simetrias simples para $\mathfrak{s u}(2)$-simetrias diagonais. Considerando as cadeias sobreviventes resultando da fase anterior que terminam em uma soma direta de $p$ cópias de $\mathfrak{s u}(2)$, quebramos estas de forma diagonal. Observe que, a priori, há $\left(\begin{array}{l}p \\ 2\end{array}\right)$ possibilidades de subalgebras de tipo diagonal. Porém este número pode ser reduzido quando percebermos que a distribuição de multipletos fornecida pelas quebras anteriores é invariante sob certas permutações dos ideais $\mathfrak{s u}(2)$.

Fase 3: Quebra das $\mathfrak{s u ( 2 ) - s i m e t r i a s . ~ C o n s i d e r a n d o ~ a s ~ c a d e i a s ~ s o b r e v i v e n t e s ~ r e - ~}$ sultando das fases anteriores, passamos a quebrar os ideais $\mathfrak{s u}(2)$. 
Quebra de Simetrias no Código Genético

A possibilidade de agrupamento em famílias é baseada na observação de que o processo de quebra de simetria é comutativo, no seguinte sentido. Considere duas cadeias descendentes

$$
\mathfrak{g} \supset \mathfrak{g}_{1} \supset \mathfrak{g}_{2} \supset \ldots \supset \mathfrak{g}_{r} \supset \mathfrak{h}
$$

e

$$
\mathfrak{g} \supset \mathfrak{g}_{1}^{\prime} \supset \mathfrak{g}_{2}^{\prime} \supset \ldots \supset \mathfrak{g}_{r}^{\prime} \supset \mathfrak{h}
$$

de subalgebras com a mesma álgebra inicial $\mathfrak{g}$ e a mesma álgebra final $\mathfrak{h}$ : então as distribuições de multipletos sob $\mathfrak{h}$ obtidos de uma dada representação de $\mathfrak{g}$ através das duas cadeias são idênticas.

Passaremos agora a discutir a fase 3 com mais detalhes, seguindo de perto a apresentação em [23], tendo em vista que este manuscrito ainda não foi publicado. Dada uma álgebra de Lie simples $\mathfrak{g}$, junto com uma cadeia descendente

$$
\mathfrak{g} \supset \mathfrak{g}_{1} \supset \mathfrak{g}_{2} \supset \ldots
$$

de subalgebras $\mathfrak{g}_{1}, \mathfrak{g}_{2}, \ldots$, a distribuição de multipletos obtida pela sucessiva decomposição de uma dada representação irredutível de $\mathfrak{g}$ pode ser codificada no espectro de um único operador $H$ : ele pode ser definido como uma combinação linear genérica de operadores de Casimir $C_{j}$ das subalgebras simples de $\mathfrak{g}$ que constituem as subalgebras semisimples $\mathfrak{g}_{1}, \mathfrak{g}_{2}, \ldots$ que aparecem na cadeia:

$$
H=\sum_{j} \lambda_{j} C_{j} .
$$

De fato, devido às relações de inclusão entre as $\mathfrak{g}_{j}$, os operadores de Casimir $C_{j}$ comutam entre si, e para uma escolha genérica dos coeficientes $\lambda_{j}$, os autoespaços do operador $H$ coincidem com os autoespaços comuns do conjunto dos operadores de Casimir $C_{j}$, que por sua vez são apenas os subespaços irredutíveis relativos à menor (última) subalgebra na cadeia. Chamaremos este operador $H$ de Hamiltoniano, em analogia ao operador encontrado na física.

Para sermos mais específicos, lebremos que depois que as primeiras duas fases do processo de quebra de simetria foram completadas, a última subalgebra na cadeia é uma soma direta de $\mathfrak{s u}(2)$-subalgebras e portanto o Hamiltoniano $H$ associado a este estágio pode ser escrito da forma

$$
H=\sum_{j} \lambda_{j} C_{j}+\sum_{k=1}^{p} \alpha_{k} \boldsymbol{L}_{k}^{2}
$$

onde $p$, o número total de $\mathfrak{s u}(2)$-subalgebras que aparecem no final da cadeia, varia de 1 até o posto de $\mathfrak{g}$, dependendo da cadeia. Os $C_{j}$ são agora operadores de Casimir associados às álgebras simples $\neq \mathfrak{s u}(2)$ que constituem as subalgebras semisimples $\mathfrak{g}_{1}, \mathfrak{g}_{2}, \ldots$ que aparecem 
na cadeia, enquanto que $\boldsymbol{L}_{k}^{2}=L_{k, x}^{2}+L_{k, y}^{2}+L_{k, z}^{2}$ é o operador de Casimir padrão da $k$-ésima $\mathfrak{s u}(2)$-subalgebra $(1 \leqslant k \leqslant p)$.

A terceira fase do processo de quebra de simetria, que envolve a quebra de uma ou várias $\mathfrak{s u}(2)$-subalgebras, será implementada por um Hamiltoniano da forma:

$$
H=\sum_{j} \lambda_{j} C_{j}+\sum_{k=1}^{p} \alpha_{k} \boldsymbol{L}_{k}^{2}+\sum_{k=1}^{p} \beta_{k} L_{k, z}^{2}+\sum_{k=1}^{p} \gamma_{k} L_{k, z}
$$

Para explicar o efeito destes novos termos, consideraremos o caso mais simples com apenas uma cópia de $\mathfrak{s u}(2)$. Levando em conta o fato de que qualquer representação irredutível de $\mathfrak{s u}(2)$, caracterizada por seu spin $s$ (correspondendo ao peso máximo $2 s$, que pode ser qualquer valor inteiro não-negativo), forma um espaço de dimensão $2 s+1$, sobre o qual o operador de Casimir padrão $\boldsymbol{L}^{2}$ de $\mathfrak{s u}(2)$ tem o valor próprio $s(s+1)$, enquanto que o operador $L_{z}$ tem $2 s+1$ valores próprios distintos $m=-s, \ldots, s$, vemos que

a) o operador $L_{z}$ providencia uma cisão completa do multipleto de dimensão $2 s+1$ em $2 s+1$ singletos, enquanto que

b) o operador $L_{z}^{2}$ providencia uma cisão mais suave do multipleto de dimensão $2 s+1 \mathrm{em}$ $s$ dubletos e um singleto se $s$ for inteiro, ou em $s$ dubletos se $s$ for semi-inteiro.

Somente a primeira possibilidade corresponde a uma genuina quebra de simetria no nivel de álgebras de Lie: da álgebra de Lie $\mathfrak{s u}(2)$ para sua subalgebra maximal $\mathfrak{u}(1)$, que é nada mais que uma álgebra de Lie abeliana unidimensional. Foi observado no entanto que as duas possibilidades possuem uma interpretação natural em termos de uma quebra genuina de simetria no nivel de grupos de Lie: do grupo (conexo) $S U(2)$ para

a) seu subgrupo maximal conexo $U(1) \cong S O(2)$

b) seu subgrupo maximal não-conexo $\mathbb{Z}_{2} \times U(1) \cong O(2)$, um subgrupo de $S U(2)$ formado por dois circulos:

$$
\mathbb{Z}_{2} \times U(1)=\left\{\left(\begin{array}{cc}
e^{i \alpha} & 0 \\
0 & e^{-i \alpha}
\end{array}\right) \mid \alpha \in \mathbb{R}\right\} \cup\left\{\left(\begin{array}{cc}
0 & e^{i \beta} \\
e^{-i \beta} & 0
\end{array}\right) \mid \beta \in \mathbb{R}\right\} .
$$

Note que $\mathbb{Z}_{2} \times U(1)$ é gerado por $U(1)$ juntamente com a matriz

$$
\left(\begin{array}{ll}
0 & 1 \\
1 & 0
\end{array}\right)
$$

que é exatamente o gerador do grupo de Weyl de $S U(2)$. Por abuso de linguagem, nos referiremos a estas duas reduções da $\mathfrak{s u}(2)$-simetria, em forma abreviada, por $\mathfrak{s o}(2)$-simetria 
Quebra de Simetrias no Código Genético

e $\mathfrak{o}(2)$-simetria, respectivamente (apesar de que $S O(2)$-simetria e $O(2)$-simetria seria mais apropriado).

O último ingrediente importante no processo de quebra de simetria é que permitiremos que os coeficientes $\gamma_{k}$ do Hamiltoniano $H$ dado acima sejam polinômios nos operadores de Casimir padrão $\boldsymbol{L}_{k}^{2}$ das subalgebras $\mathfrak{s u}(2)$, e não apenas constantes. Isto possibilita uma forma de interrupção muito específica no processo de quebra de simetria no último passo, pois os multipletos do penúltimo estágio que normalmente se dividiriam no último passo permanecerão intactos se (e somente se) seus $\mathfrak{s u}(2)$-índices são tais que os coeficientes $\gamma_{k}$ correspondentes se anulam. Com este formalismo podemos implementar o conceito de congelamento de alguns multipletos no último passo da quebra. Por outro lado, perdemos a acima mencionada comutatividade no processo de quebra, pois se a quebra através de duas cadeias

$$
\mathfrak{g} \supset \mathfrak{g}_{1} \supset \mathfrak{g}_{2} \supset \ldots \supset \mathfrak{g}_{r} \supset \mathfrak{h}
$$

e

$$
\mathfrak{g} \supset \mathfrak{g}_{1}^{\prime} \supset \mathfrak{g}_{2}^{\prime} \supset \ldots \supset \mathfrak{g}_{r}^{\prime} \supset \mathfrak{h}
$$

envolve congelamento no último passo (de $\mathfrak{g}_{r}$ para $\mathfrak{h}$ e de $\mathfrak{g}_{r}^{\prime}$ para $\mathfrak{h}$ ), o resultado não será necessariamente o mesmo. Este fato aumenta consideravelmente o número de casos a serem analisados. Portanto, precisamos novamente de critérios que possam minimizar o número de possibilidades a serem investigadas na terceira fase. Observou-se que tais critérios podem ser baseados em algumas regras gerais que se referem à questão de como surgem os 3 sextetos e os 2 tripletos do código genético. Estas regras são geralmente chamadas de regras de geração de tripletos/sextetos.

Mais especificamente, suponhamos que ao final da fase 2 do processo de quebra de simetria, foi gerada uma cadeia terminando numa soma direta de $p$ cópias de $\mathfrak{s u}(2)$, com uma distribuição de multipletos cada um dos quais pode ser escrito da forma $\left(k_{1}, \ldots, k_{p}\right)$ com inteiros não-negativos $k_{1}, \ldots, k_{p}$. No caso das cadeias para $B_{6}$ e $D_{7}$, observamos que $p$ varia entre 2 e 5 (os valores teóricamente possíveis 1 e 6 correspondem a cadeias que podem ser imediatamente descartadas). Consideremos então uma redução da simetria do multipleto $\left(k_{1}, \ldots, k_{p}\right)$ de dimensão $\left(k_{1}+1\right) \ldots\left(k_{p}+1\right)$, de $\mathfrak{s u}_{1}(2) \oplus \ldots \oplus \mathfrak{s u}_{j}(2) \oplus \ldots \oplus \mathfrak{s u}_{p}(2)$ para $\mathfrak{s u}_{1}(2) \oplus \ldots \oplus \mathfrak{o}_{j}(2) \oplus \ldots \oplus \mathfrak{s u}_{p}(2)$ ou para $\mathfrak{s u}_{1}(2) \oplus \ldots \oplus \mathfrak{s o}_{j}(2) \oplus \ldots \oplus \mathfrak{s u}_{p}(2)$. No segundo caso, ele se divide em soma direta de $k_{j}+1$ sub-multipletos de dimensão $\left(k_{1}+1\right) \ldots\left(k_{j-1}\right)\left(k_{j+1}\right) \ldots\left(k_{p}+1\right)$, enquanto que no segundo caso, ele se divide em uma soma direta de $\left(k_{j}+1\right) / 2$ sub-multipletos de dimensão $2\left(k_{1}+1\right) \ldots\left(k_{j-1}+1\right)$ $\left(k_{j+1}+1\right) \ldots\left(k_{p}+1\right)$ se $k_{j}$ é impar e $k_{j} / 2$ sub-multipletos de dimensão $2\left(k_{1}+1\right) \ldots\left(k_{j-1}\right)$ $\left(k_{j+1}\right) \ldots\left(k_{p}+1\right)$ mais 1 sub-multipleto de dimensão $\left(k_{1}+1\right) \ldots\left(k_{j-1}\right)\left(k_{j+1}\right) \ldots\left(k_{p}+1\right)$ se $k_{j}$ é par. Repetindo o processo, concluímos que qualquer quebra do multipleto $\left(k_{1}, \ldots, k_{p}\right)$ leva a uma distribuição de sub-multipletos cujas dimensões são produtos de certos números $k_{j}+1$ que correspondem aos $\mathfrak{s u}(2)$ que permaneceram intactos e, quando ocorre uma quebra 
envolvendo $\mathfrak{o}(2)$, alguma potência de 2. Em particular, sub-multipletos cuja dimensão é um múltiplo de 3 podem aparecer apenas se pelo menos um dos números $k_{j}+1$ for um múltiplo de 3 (e o correspondente $\mathfrak{s u}_{j}(2)$ permanecer intacto); ademais, tripletos (sextetos) podem aparecer apenas se pelo menos um deles é igual a 3 (3 ou 6). Portanto temos

Regra 1: Sub-multipletos cuja dimensão é um múltiplo de 3 podem aparecer apenas como resultado da quebra de um multipleto $\left(k_{1}, \ldots, k_{p}\right)$ cuja dimensão é um múltiplo de 3 , isto é, pelo menos um dos inteiros $k_{1}, \ldots, k_{p}$ deve ser igual a $2 \bmod 3$. Mais especficamente, os tripletos provêm de multipletos $\left(k_{1}, \ldots, k_{p}\right)$ onde pelo menos um dos inteiros $k_{1}, \ldots, k_{p}$ é igual a 2 , equanto que os sextetos provêm de multipletos $\left(k_{1}, \ldots, k_{p}\right)$ onde pelo menos um dos inteiros $k_{1}, \ldots, k_{p}$ é igual a 2 ou 5 .

Regra 2: A quebra de um multipleto cuja dimensão é um múltiplo de 3 resulta numa coleção de sub-multipletos cuja dimensão é um múltiplo de 3 ou para todos ou para nenhum dos sub-multipletos da coleção.

Na tabela 2.21 abaixo, listamos os multipletos $\left(k_{1}, \ldots, k_{p}\right)$ que realmente aparecem nas tabelas para $B_{6}$ e $D_{7}$, juntamente com suas possíveis quebras - exceto para aqueles que não produzem nenhum sub-multipleto cuja dimensão é um múltiplo de 3 ou que não conseguem eliminar todos os sub-multipletos de dimensão $>6$. Sem perda de generalidade, assumimos que $k_{1}$ é igual a 2 ou 5 , e quando escrevemos $\left(k_{1}, 0, \ldots\right),\left(k_{1}, k_{2}, 0, \ldots\right),\left(k_{1}, k_{2}, k_{3}, 0, \ldots\right)$, etc., os pontos indicam que os $k_{j}$ restantes são iguais a 0.

Um método global de se levar em consideração os sextetos e os tripletos contidos numa dada distribuição de multipletos é através do número de trialidade, definido por

$$
d_{3}=\begin{gathered}
\text { soma das dimensões de todos os multipletos } \\
\text { cuja dimensão é um múltiplo de } 3 .
\end{gathered}
$$

Com esta notação, podemos formular um último critério, baseado na observação de que quando se percorre uma dada cadeia, dentro da fase 3 , o número de trialidade $d_{3}$ não pode crescer: isto é uma consquência direta das regras 1 e 2 formuladas acima. Observando que o valor de $d_{3}$ na distribuição final de multipletos do código genético é 24 , concluímos que todas as cadeias sobreviventes que, após a conclusão das fases 1 e 2 do processo de quebra de simetria ou em algum outro ponto durante a fase 3, exceto talvez o último passo, violam a estimativa

$$
d_{3} \geqslant 24
$$

não serão capazes de gerar os sextetos e tripletos do código genético e podem portanto ser descartadas. A ressalva quanto ao último passo se deve ao fato de que uma perda excessiva de sextetos e tripletos durante esse passo pode ser evitada através do congelamento. 


\begin{tabular}{|c|c|}
\hline Multipletos & $\begin{array}{c}\text { Possível Distribuição } \\
\text { de sub-multipletos }\end{array}$ \\
\hline$(5,0, \ldots)_{6}$ & 1 sexteto \\
\hline$(2,0, \ldots)_{3}$ & 1 tripleto \\
\hline$(8,1,0, \ldots)_{18}$ & - \\
\hline$(5,1,0, \ldots)_{12}$ & 2 sextetos \\
\hline \multirow[t]{2}{*}{$(2,6,0, \ldots)_{21}$} & 3 sextetos e 1 tripleto \\
\hline & 7 tripletos \\
\hline \multirow[t]{2}{*}{$(2,5,0, \ldots)_{18}$} & 3 sextetos \\
\hline & 6 tripletos \\
\hline \multirow[t]{2}{*}{$(2,4,0, \ldots)_{15}$} & 2 sextetos e 1 tripleto \\
\hline & 5 tripletos \\
\hline \multirow[t]{2}{*}{$(2,3,0, \ldots)_{12}$} & 2 sextetos \\
\hline & 4 tripletos \\
\hline \multirow[t]{2}{*}{$(2,2,0, \ldots)_{9}$} & 1 sexteto e 1 tripleto \\
\hline & 3 tripletos \\
\hline \multirow[t]{2}{*}{$(2,1,0, \ldots)_{6}$} & 1 sexteto \\
\hline & 2 tripletos \\
\hline \multirow[t]{2}{*}{$(2,1,3,0, \ldots)_{24}$} & 4 sextetos \\
\hline & 8 tripletos \\
\hline \multirow{3}{*}{$(2,1,2,0, \ldots)_{18}$} & 3 sextetos \\
\hline & 2 sextetos e 2 tripletos \\
\hline & 6 tripletos \\
\hline \multirow[t]{2}{*}{$(2,1,1,0, \ldots)_{12}$} & 2 sextetos \\
\hline & 4 tripletos \\
\hline
\end{tabular}

Tabela 2.21: Multipletos que aparecem em $B_{6}$ e $D_{7}$. 
Finalmente, tendo explicado toda a metodologia, podemos apresentar os resultados finais da análise das cadeias provindo de $B_{6}=\mathfrak{s o}(13)$ e $D_{7}=\mathfrak{s o}(14)$. Podemos distinguir dois tipos de cadeias: as cadeias simples onde todas as reduções a subalgebras são do tipo simples, que já foram analisadas em [3], e as cadeias diagonais onde as reduções a subalgebras podem ser do tipo simples ou do tipo diagonal, cuja análise constitui a contribuição nova do presente trabalho. Após a conclusão das duas primeiras fases obtemos 15 cadeias sobreviventes no caso de $B_{6}$, sendo 2 simples e 13 diagonais, e 39 cadeias sobreviventes no caso de $D_{7}$, sendo 9 simples e 30 diagonais. Destas, não sobrevive nenhuma cadeia de $B_{6}$, enquanto que restam 5 cadeias de $D_{7}$, uma simples e quatro diagonais, que após a conclusão da terceira fase fornecem um total de 8 cadeias com a distribuição de multipletos do código genético e que serão apresentadas a seguir. Cada uma está dividida em duas partes onde a primeira parte corresponde à quebra durante as duas primeiras fases e a segunda parte corresponde à terceira fase. Nas tabelas que representam a terceira fase as células que estão rachuradas indicam os multipletos que foram congelados no último passo do processo de quebra. A última linha de cada tabela indica o número de subespaços invariantes (com a abreviação s.r.) que aparece a cada estágio da quebra.

1) $D_{7} \supset B_{3} \oplus B_{3} \supset B_{3} \oplus G_{2} \supset G_{2} \oplus G_{2} \supset G_{2} \oplus A_{1} \oplus A_{1} \supset A_{1} \oplus A_{1} \oplus A_{1} \oplus A_{1}$.

\begin{tabular}{|c|c|c|c|c|c|c|c|c|c|c|c|}
\hline \multicolumn{2}{|l|}{$D_{7}$} & \multicolumn{2}{|c|}{$B_{3} \oplus B_{3}$} & \multicolumn{2}{|c|}{$B_{3} \oplus G_{2}$} & \multicolumn{2}{|c|}{$G_{2} \oplus G_{2}$} & \multicolumn{2}{|c|}{$G_{2} \oplus\left(A_{1}\right)^{2}$} & \multicolumn{2}{|l|}{$\left(A_{1}\right)^{4}$} \\
\hline Peso & $d$ & Peso & $d$ & Peso & $d$ & Peso & $d$ & Peso & $d$ & Peso & $d$ \\
\hline \multirow[t]{9}{*}{ (0000010) } & \multirow[t]{9}{*}{64} & \multirow[t]{9}{*}{$(001,001)$} & \multirow[t]{9}{*}{64} & \multirow[t]{6}{*}{$(001,01)$} & \multirow[t]{6}{*}{56} & \multirow[t]{4}{*}{$(01,01)$} & \multirow[t]{4}{*}{49} & \multirow[t]{2}{*}{$(01,1,1)$} & \multirow[t]{2}{*}{28} & $(1,1,1,1)$ & 16 \\
\hline & & & & & & & & & & $(0,2,1,1)$ & 12 \\
\hline & & & & & & & & \multirow[t]{2}{*}{$(01,0,2)$} & \multirow[t]{2}{*}{21} & $(1,1,0,2)$ & 12 \\
\hline & & & & & & & & & & $(0,2,0,2)$ & 9 \\
\hline & & & & & & \multirow[t]{2}{*}{$(00,01)$} & \multirow[t]{2}{*}{7} & $(00,1,1)$ & 4 & $(0,0,1,1)$ & 4 \\
\hline & & & & & & & & $(00,0,2)$ & 3 & $(0,0,0,2)$ & 3 \\
\hline & & & & \multirow[t]{3}{*}{$(001,00)$} & \multirow[t]{3}{*}{8} & \multirow[t]{2}{*}{$(01,00)$} & \multirow[t]{2}{*}{7} & \multirow[t]{2}{*}{$(01,0,0)$} & \multirow[t]{2}{*}{7} & $(1,1,0,0)$ & 4 \\
\hline & & & & & & & & & & $(0,2,0,0)$ & 3 \\
\hline & & & & & & $(00,00)$ & 1 & $(00,0,0)$ & 1 & $(0,0,0,0)$ & 1 \\
\hline \multicolumn{2}{|l|}{1 S.I. } & \multicolumn{2}{|l|}{1 S.I. } & \multicolumn{2}{|l|}{2 S.I. } & \multicolumn{2}{|c|}{4 S.I. } & \multicolumn{2}{|l|}{6 S.I. } & \multicolumn{2}{|l|}{9 S.I. } \\
\hline
\end{tabular}

Tabela 2.22: Neste caso obtemos a distribuição de multipletos do código genético quebrando primeiro o primeiro $\mathfrak{s u}(2)$ para $\mathfrak{s o}(2)$, depois o quarto $\mathfrak{s u}(2)$ para $\mathfrak{o}(2)$ e finalmente este $\mathfrak{o}(2)$ para $\mathfrak{s o}(2)$. Esta distribuição está apresentada na tabela seguinte. 


\begin{tabular}{|c|c|c|c|c|c|}
\hline \multicolumn{2}{|l|}{$\left(A_{1}\right)^{4}$} & \multicolumn{2}{|c|}{$\left(L_{1, z}, L_{4, z}^{2}\right)$} & \multicolumn{2}{|c|}{$\left(L_{1, z}, L_{4, z}^{2}, L_{4, z}\right)$} \\
\hline Peso & $d$ & Peso & $d$ & Peso & $d$ \\
\hline \multirow[t]{4}{*}{$(1,1,1,1)$} & \multirow[t]{4}{*}{16} & \multirow[t]{2}{*}{$(+1,1,1, \pm 1)$} & \multirow[t]{2}{*}{8} & $(+1,1,1,+1)$ & 4 \\
\hline & & & & $(+1,1,1,-1)$ & 4 \\
\hline & & \multirow[t]{2}{*}{$(-1,1,1, \pm 1)$} & \multirow[t]{2}{*}{8} & $(-1,1,1,+1)$ & 4 \\
\hline & & & & $(-1,1,1,-1)$ & 4 \\
\hline \multirow[t]{2}{*}{$(0,2,1,1)$} & \multirow[t]{2}{*}{8} & \multirow[t]{2}{*}{$(0,2,1, \pm 1)$} & \multirow[t]{2}{*}{8} & $(0,2,1,+1)$ & 4 \\
\hline & & & & $(0,2,1,+1)$ & 4 \\
\hline \multirow[t]{6}{*}{$(1,1,0,2)$} & \multirow[t]{6}{*}{12} & \multirow{2}{*}{$(+1,1,0, \pm 2)$} & \multirow[t]{2}{*}{4} & $(+1,1,0,+2)$ & 2 \\
\hline & & & & $(+1,1,0,-2)$ & 2 \\
\hline & & $(+1,1,0,0)$ & 2 & $(+1,1,0,0)$ & 2 \\
\hline & & \multirow[t]{2}{*}{$(-1,1,0, \pm 2)$} & \multirow[t]{2}{*}{4} & $(-1,1,0,+2)$ & 2 \\
\hline & & & & $(-1,1,0,-2)$ & 2 \\
\hline & & $(-1,1,0,0)$ & 2 & $(-1,1,0,0)$ & 2 \\
\hline \multirow{3}{*}{$(0,2,0,2)$} & \multirow[t]{3}{*}{9} & $(0,2,0, \pm 2)$ & 6 & $(0,2,0,+2)$ & 3 \\
\hline & & & & $(0,2,0,-2)$ & 3 \\
\hline & & $(0,2,0,0)$ & 3 & $(0,2,0,0)$ & 3 \\
\hline \multirow[t]{2}{*}{$(0,1,1,1)$} & \multirow[t]{2}{*}{4} & \multirow[t]{2}{*}{$(0,1,1, \pm 1)$} & \multirow[t]{2}{*}{4} & $(0,1,1,+1)$ & 2 \\
\hline & & & & $(0,1,1,-1)$ & 2 \\
\hline \multirow[t]{3}{*}{$(0,0,0,2)$} & \multirow[t]{3}{*}{3} & \multirow[t]{2}{*}{$(0,0,0, \pm 2)$} & \multirow[t]{2}{*}{2} & $(0,0,0,+2)$ & 1 \\
\hline & & & & $(0,0,0,-2)$ & 1 \\
\hline & & $(0,0,0,0)$ & 1 & $(0,0,0,0)$ & 1 \\
\hline \multirow[t]{2}{*}{$(1,1,0,0)$} & \multirow[t]{2}{*}{4} & $(+1,1,0,0)$ & 2 & $(+1,1,0,0)$ & 2 \\
\hline & & $(-1,1,0,0)$ & 2 & $(-1,1,0,0)$ & 2 \\
\hline$(0,2,0,0)$ & 3 & $(0,2,0,0)$ & 3 & $(0,2,0,0)$ & 3 \\
\hline$(0,0,0,0)$ & 1 & $(0,0,0,0)$ & 1 & $(0,0,0,0)$ & 1 \\
\hline \multicolumn{2}{|l|}{9 S.I. } & \multicolumn{2}{|l|}{16 S.I. } & 24 S.I. & \\
\hline
\end{tabular}


2) $D_{7} \supset B_{3} \oplus B_{3} \supset B_{3} \oplus G_{2} \supset G_{2} \oplus G_{2} \supset G_{2} \oplus A_{1} \oplus A_{1} \supset A_{1} \oplus A_{1} \oplus A_{1} \oplus A_{1} \supset\left(A_{1}\right)_{13} \oplus A_{1} \oplus A_{1}$.

\begin{tabular}{|c|c|c|c|c|c|c|c|c|c|c|c|c|c|}
\hline \multicolumn{2}{|l|}{$D_{7}$} & \multicolumn{2}{|c|}{$B_{3} \oplus B_{3}$} & \multicolumn{2}{|c|}{$B_{3} \oplus G_{2}$} & \multicolumn{2}{|c|}{$G_{2} \oplus G_{2}$} & \multicolumn{2}{|c|}{$G_{2} \oplus\left(A_{1}\right)^{2}$} & \multicolumn{2}{|l|}{$\left(A_{1}\right)^{4}$} & \multicolumn{2}{|c|}{$\left(A_{1}\right)^{3}$} \\
\hline Peso & $d$ & Peso & $d$ & Peso & $d$ & Peso & $d$ & Peso & $d$ & Peso & $d$ & Peso & $d$ \\
\hline \multirow[t]{10}{*}{ (0000010) } & \multirow[t]{10}{*}{64} & \multirow[t]{10}{*}{$(001,001)$} & \multirow[t]{10}{*}{64} & \multirow[t]{7}{*}{$(001,01)$} & \multirow[t]{7}{*}{56} & \multirow[t]{5}{*}{$(01,01)$} & \multirow[t]{5}{*}{49} & \multirow[t]{3}{*}{$(01,1,1)$} & \multirow[t]{3}{*}{28} & \multirow[t]{2}{*}{$(1,1,1,1)$} & \multirow[t]{2}{*}{16} & $(2,1,1)$ & 12 \\
\hline & & & & & & & & & & & & $(0,1,1)$ & 4 \\
\hline & & & & & & & & & & $(0,2,1,1)$ & 12 & $(1,2,1)$ & 12 \\
\hline & & & & & & & & \multirow[t]{2}{*}{$(01,0,2)$} & \multirow[t]{2}{*}{21} & $(1,1,0,2)$ & 12 & $(1,1,2)$ & 12 \\
\hline & & & & & & & & & & $(0,2,0,2)$ & 9 & $(0,2,2)$ & 9 \\
\hline & & & & & & \multirow[t]{2}{*}{$(00,01)$} & \multirow[t]{2}{*}{7} & $(00,1,1)$ & 4 & $(0,0,1,1)$ & 4 & $(1,0,1)$ & 4 \\
\hline & & & & & & & & $(00,0,2)$ & 3 & $(0,0,0,2)$ & 3 & $(0,0,2)$ & 3 \\
\hline & & & & \multirow[t]{3}{*}{$(001,00)$} & \multirow[t]{3}{*}{8} & \multirow[t]{2}{*}{$(01,00)$} & \multirow[t]{2}{*}{7} & \multirow[t]{2}{*}{$(01,0,0)$} & \multirow[t]{2}{*}{7} & $(1,1,0,0)$ & 4 & $(1,1,0)$ & 4 \\
\hline & & & & & & & & & & $(0,2,0,0)$ & 3 & $(0,2,0)$ & 3 \\
\hline & & & & & & $(00,00)$ & 1 & $(00,0,0)$ & 1 & $(0,0,0,0)$ & 1 & $(0,0,0)$ & 1 \\
\hline \multicolumn{2}{|l|}{1 S.I. } & \multicolumn{2}{|l|}{1 S.I. } & \multicolumn{2}{|l|}{2 S.I. } & \multicolumn{2}{|c|}{4 S.I. } & \multicolumn{2}{|l|}{6 S.I. } & \multicolumn{2}{|l|}{9 S.I. } & \multicolumn{2}{|c|}{10 S.I. } \\
\hline
\end{tabular}

Tabela 2.23: Neste caso há duas possibilidades de se obter a distribuição de multipletos do código genético:

a) quebrando primeiro o primeiro $\mathfrak{s u}(2)$ para $\mathfrak{s o}(2)$, depois o segundo $\mathfrak{s u}(2)$ para $\mathfrak{o}(2)$ e finalmente este $\mathfrak{o}(2)$ para $\mathfrak{s o}(2)$.

b) quebrando primeiro o primeiro $\mathfrak{s u}(2)$ para $\mathfrak{s o}(2)$, depois o segundo $\mathfrak{s u}(2)$ para $\mathfrak{o}(2)$ e finalmente o terceiro $\mathfrak{s u}(2)$ para $\mathfrak{s o}(2)$.

Ambas as possibilidades estão apresentadas nas duas tabelas seguintes. 


\begin{tabular}{|c|c|c|c|c|c|c|c|}
\hline \multicolumn{2}{|c|}{$\left(A_{1}\right)^{3}$} & \multicolumn{2}{|l|}{$L_{1, z}$} & \multicolumn{2}{|c|}{$\left(L_{1, z}, L_{2, z}^{2}\right)$} & \multicolumn{2}{|c|}{$\left(L_{1, z}, L_{2, z}^{2}, L_{2, z}\right)$} \\
\hline Peso & $d$ & Peso & $d$ & Peso & $d$ & Peso & $d$ \\
\hline \multirow[t]{6}{*}{$(2,1,1)$} & \multirow[t]{6}{*}{12} & $(+2,1,1)$ & 4 & $(+2, \pm 1,1)$ & 4 & $(+2,+1,1)$ & 2 \\
\hline & & & & & & $(+2,-1,1)$ & 2 \\
\hline & & $(-2,1,1)$ & 4 & $(-2, \pm 1,1)$ & 4 & $(-2,+1,1)$ & 2 \\
\hline & & & & & & $(-2,-1,1)$ & \\
\hline & & $(0,1,1)$ & 4 & $(0, \pm 1,1)$ & 4 & $(0,+1,1)$ & 2 \\
\hline & & & & & & $(0,-1,1)$ & \\
\hline \multirow[t]{2}{*}{$(0,1,1)$} & \multirow[t]{2}{*}{4} & \multirow[t]{2}{*}{$(0,1,1)$} & \multirow[t]{2}{*}{4} & \multirow[t]{2}{*}{$(0, \pm 1,1)$} & \multirow[t]{2}{*}{4} & $(0,+1,1)$ & 2 \\
\hline & & & & & & $(0,-1,1)$ & 2 \\
\hline \multirow[t]{6}{*}{$(1,2,1)$} & \multirow[t]{6}{*}{12} & \multirow[t]{3}{*}{$(+1,2,1)$} & \multirow[t]{3}{*}{6} & $(+1, \pm 2,1)$ & 4 & $(+1,+2,1)$ & 2 \\
\hline & & & & & & $(+1,-$ & \\
\hline & & & & $(+1,0,1)$ & 2 & $(+1,0,1)$ & 2 \\
\hline & & \multirow{3}{*}{$(-1,2,1)$} & \multirow[t]{3}{*}{6} & $(-1, \pm 2,1)$ & 4 & $(-1,+2,1)$ & 2 \\
\hline & & & & & & $(-1$, & ? \\
\hline & & & & $(-1)$ & 2 & $(-1,0,1)$ & \\
\hline \multirow[t]{4}{*}{$(1,1,2)$} & \multirow[t]{4}{*}{12} & $(+1,1,2)$ & 6 & $(+1, \pm 1,2)$ & 6 & $(+1,+1,2)$ & 3 \\
\hline & & & & & & $(+1,-1,2)$ & \\
\hline & & $(-1,1,2)$ & 6 & $(-1, \pm 1,2)$ & 6 & $(-1,1,2)$ & \\
\hline & & & & & & $(-1,-1,2)$ & 3 \\
\hline \multirow[t]{3}{*}{$(0,2,2)$} & \multirow[t]{3}{*}{9} & \multirow[t]{3}{*}{$(0,2,2)$} & \multirow[t]{3}{*}{9} & $(0, \pm 2,2)$ & 6 & $(0,+2,2)$ & 3 \\
\hline & & & & & & $(0,-2,2)$ & \\
\hline & & & & $(0,0,2)$ & 3 & $(0,0,2)$ & 3 \\
\hline \multirow[t]{2}{*}{$(1,0,1)$} & \multirow[t]{2}{*}{4} & $(+1,0,1)$ & 2 & $(+1,0,1)$ & 2 & $(+1,0,1)$ & 2 \\
\hline & & $(-1,0,1)$ & 2 & $(-1,0,1)$ & 2 & $(-1,0,1)$ & 2 \\
\hline$(0,0,2)$ & 3 & $(0,0,2)$ & 3 & $(0,0,2)$ & 3 & $(0,0,2)$ & 3 \\
\hline \multirow[t]{4}{*}{$(1,1,0)$} & \multirow[t]{4}{*}{4} & $(+1,1,0)$ & 2 & $(+1, \pm 1,0)$ & 2 & $(+1,+1,0)$ & 1 \\
\hline & & & & & & $(+1,-1,0)$ & \\
\hline & & $(-1,1,0)$ & 2 & $(-1, \pm 1,0)$ & 2 & $(-1,1,0)$ & 1 \\
\hline & & & & & & $(-1,1,0)$ & 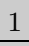 \\
\hline$(0,2,0)$ & 3 & $(0,2,0)$ & 3 & $(0, \pm 2,0)$ & 2 & $(0,+2,0)$ & 1 \\
\hline & & & & & & $(0,-2,0)$ & 1 \\
\hline & & & & $(0,0,0)$ & 1 & $(0,0,0)$ & 1 \\
\hline$(0,0,0)$ & 1 & $(0,0,0)$ & 1 & $(0,0,0)$ & 1 & $(0,0,0)$ & 1 \\
\hline $10 \mathrm{~s}$. & & 16 S.I. & & 20 S.I. & & 32 S.I. & \\
\hline
\end{tabular}




\begin{tabular}{|c|c|c|c|c|c|c|c|}
\hline \multicolumn{2}{|c|}{$\left(A_{1}\right)^{3}$} & \multicolumn{2}{|l|}{$L_{1, z}$} & \multicolumn{2}{|c|}{$\left(L_{1, z}, L_{2, z}^{2}\right)$} & \multicolumn{2}{|c|}{$\left(L_{1, z}, L_{2, z}^{2}, L_{3, z}\right)$} \\
\hline Peso & $d$ & Peso & $d$ & Peso & $d$ & Peso & $d$ \\
\hline \multirow[t]{3}{*}{$(2,1,1)$} & \multirow[t]{3}{*}{12} & $(+2,1,1)$ & 4 & $(+2, \pm 1,1)$ & 4 & $\begin{array}{l}(+2, \pm 1,+1) \\
(+2, \pm 1,-1)\end{array}$ & 2 \\
\hline & & $(-2,1,1)$ & 4 & $(-2, \pm 1,1)$ & 4 & $\begin{array}{l}(-2, \pm 1,+1) \\
(-2, \pm 1,-1)\end{array}$ & 2 \\
\hline & & $(0,1,1)$ & 4 & $(0, \pm 1,1)$ & 4 & $\begin{array}{l}(0, \pm 1,+1) \\
(0, \pm 1,-1)\end{array}$ & $\begin{array}{l}2 \\
2\end{array}$ \\
\hline \multirow[t]{2}{*}{$(0,1,1)$} & \multirow[t]{2}{*}{4} & \multirow[t]{2}{*}{$(0,1,1)$} & \multirow[t]{2}{*}{4} & \multirow{2}{*}{$(0, \pm 1,1)$} & \multirow[t]{2}{*}{4} & $(0, \pm 1,+1)$ & 2 \\
\hline & & & & & & $(0, \pm 1,-1)$ & 2 \\
\hline \multirow[t]{5}{*}{$(1,2,1)$} & \multirow[t]{5}{*}{12} & \multirow{2}{*}{$(+1,2,1)$} & \multirow[t]{2}{*}{6} & $(+1, \pm 2,1)$ & 4 & $(+1, \pm 2,+1)$ & 2 \\
\hline & & & & $(+1$ & 2 & $\begin{array}{c}(+1, \pm 2,-1) \\
(+1,0,+1) \\
(+1,0,-1)\end{array}$ & $\begin{array}{l}2 \\
1\end{array}$ \\
\hline & & \multirow[t]{3}{*}{$(-1,2,1)$} & \multirow[t]{3}{*}{6} & $(-1, \pm 2,1)$ & 4 & $(-1, \pm 2,+1)$ & 2 \\
\hline & & & & & 2 & $\begin{array}{c}(-1, \pm 2,-1) \\
(-1,0,+1)\end{array}$ & $\begin{array}{l}2 \\
1\end{array}$ \\
\hline & & & & & & $(-1,0,-1)$ & 1 \\
\hline \multirow[t]{4}{*}{$(1,1,2)$} & \multirow[t]{4}{*}{12} & \multirow[t]{2}{*}{$(+1,1,2)$} & \multirow[t]{2}{*}{6} & \multirow{2}{*}{$(+1, \pm 1,2)$} & \multirow[t]{2}{*}{6} & $(+1, \pm 1,+2)$ & 2 \\
\hline & & & & & & $\begin{array}{c}(+1, \pm 1,-2) \\
(+1, \pm 1,0)\end{array}$ & $\begin{array}{l}2 \\
2\end{array}$ \\
\hline & & \multirow[t]{2}{*}{$(-1,1,2)$} & \multirow[t]{2}{*}{6} & \multirow[t]{2}{*}{$(-1, \pm 1,2)$} & \multirow[t]{2}{*}{6} & $(-1, \pm 1,+2)$ & 2 \\
\hline & & & & & & $\begin{array}{c}(-1, \pm 1,-2) \\
(-1, \pm 1,0)\end{array}$ & $\begin{array}{l}2 \\
2 \\
\end{array}$ \\
\hline \multirow[t]{4}{*}{$(0,2,2)$} & 9 & $(0,2,2)$ & 9 & $(0, \pm 2,2)$ & 6 & $(0, \pm 2,+2)$ & 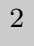 \\
\hline & & & & & & $(0, \pm 2,-2)$ & \\
\hline & & & & & & $(0, \pm 2,0)$ & 2 \\
\hline & & & & $(0,0,2)$ & 3 & $\begin{array}{c}(0,0,+2) \\
(0,0,-2) \\
(0,0,0) \\
\end{array}$ & $\begin{array}{l}1 \\
1 \\
1 \\
\end{array}$ \\
\hline$(1,0,1)$ & 4 & $(+1,0,1)$ & 2 & $(+1,0,1)$ & 2 & $(+1,0,+1)$ & 1 \\
\hline & & & & & & $(+1,0,-1)$ & \\
\hline & & $(-1,0,1)$ & 2 & $(-1,0,1)$ & 2 & $\begin{array}{l}(-1,0,+1) \\
(-1,0,-1)\end{array}$ & $\begin{array}{l}1 \\
1\end{array}$ \\
\hline$(0,0,2)$ & 3 & $(0,0,2)$ & 3 & $(0,0,2)$ & 3 & $(0,0,+2)$ & 1 \\
\hline & & & & & & $(0,0,-2)$ & 1 \\
\hline & & & & & & $(0,0,0)$ & 1 \\
\hline$(1,1,0)$ & 4 & $(+1,1,0)$ & 2 & $(+1, \pm 1,0)$ & 2 & $(+1, \pm 1,0)$ & 2 \\
\hline & & $(-1,1,0)$ & 2 & $(-1, \pm 1,0)$ & 2 & $(-1, \pm 1,0)$ & 2 \\
\hline$(0,2,0)$ & 3 & $(0,2,0)$ & 3 & $(0, \pm 2,0)$ & 2 & $(0,+2,0)$ & 4 \\
\hline & & & & $(0,0,0)$ & 1 & $(0,0,0)$ & $\begin{array}{l}1 \\
1\end{array}$ \\
\hline$(0,0,0)$ & 1 & $(0,0,0)$ & 1 & $(0,0,0)$ & 1 & $(0,0,0)$ & 1 \\
\hline $10 \mathrm{~s} . \mathrm{I}$ & & 16 S.I. & & 20 S.I. & & 39 S.I. & \\
\hline
\end{tabular}


3) $D_{7} \supset B_{3} \oplus B_{3} \supset B_{3} \oplus G_{2} \supset G_{2} \oplus G_{2} \supset G_{2} \oplus A_{1} \oplus A_{1} \supset A_{1} \oplus A_{1} \oplus A_{1} \oplus A_{1} \supset\left(A_{1}\right)_{14} \oplus A_{1} \oplus A_{1}$.

\begin{tabular}{|c|c|c|c|c|c|c|c|c|c|c|c|c|c|}
\hline \multicolumn{2}{|l|}{$D_{7}$} & \multicolumn{2}{|c|}{$B_{3} \oplus B_{3}$} & \multicolumn{2}{|c|}{$B_{3} \oplus G_{2}$} & \multicolumn{2}{|c|}{$G_{2} \oplus G_{2}$} & \multicolumn{2}{|c|}{$G_{2} \oplus\left(A_{1}\right)^{2}$} & \multicolumn{2}{|l|}{$\left(A_{1}\right)^{4}$} & \multicolumn{2}{|c|}{$\left(A_{1}\right)^{3}$} \\
\hline Peso & $d$ & Peso & $d$ & Peso & $d$ & Peso & $d$ & Peso & $d$ & Peso & $d$ & Peso & $d$ \\
\hline \multirow[t]{11}{*}{$(0000010)$} & \multirow[t]{11}{*}{64} & \multirow[t]{11}{*}{$(001,001)$} & \multirow[t]{11}{*}{64} & \multirow[t]{8}{*}{$(001,01)$} & \multirow[t]{8}{*}{56} & \multirow[t]{6}{*}{$(01,01)$} & \multirow[t]{6}{*}{49} & \multirow[t]{3}{*}{$(01,1,1)$} & \multirow[t]{3}{*}{28} & \multirow[t]{2}{*}{$(1,1,1,1)$} & \multirow[t]{2}{*}{16} & $(2,1,1)$ & 12 \\
\hline & & & & & & & & & & & & $(0,1,1)$ & 4 \\
\hline & & & & & & & & & & $(0,2,1,1)$ & 12 & $(1,2,1)$ & 12 \\
\hline & & & & & & & & \multirow[t]{3}{*}{$(01,0,2)$} & \multirow[t]{3}{*}{21} & \multirow[t]{2}{*}{$(1,1,0,2)$} & \multirow[t]{2}{*}{12} & $(3,1,0)$ & 8 \\
\hline & & & & & & & & & & & & $(1,1,0)$ & 4 \\
\hline & & & & & & & & & & $(0,2,0,2)$ & 9 & $(2,2,0)$ & 9 \\
\hline & & & & & & \multirow[t]{2}{*}{$(00,01)$} & \multirow[t]{2}{*}{7} & $(00,1,1)$ & 4 & $(0,0,1,1)$ & 4 & $(1,0,1)$ & 4 \\
\hline & & & & & & & & $(00,0,2)$ & 3 & $(0,0,0,2)$ & 3 & $(2,0,0)$ & 3 \\
\hline & & & & \multirow[t]{3}{*}{$(001,00)$} & \multirow[t]{3}{*}{8} & \multirow[t]{2}{*}{$(01,00)$} & \multirow[t]{2}{*}{7} & \multirow[t]{2}{*}{$(01,0,0)$} & \multirow[t]{2}{*}{7} & $(1,1,0,0)$ & 4 & $(1,1,0)$ & 4 \\
\hline & & & & & & & & & & $(0,2,0,0)$ & 3 & $(0,2,0)$ & 3 \\
\hline & & & & & & $(00,00)$ & 1 & $(00,0,0)$ & 1 & $(0,0,0,0)$ & 1 & $(0,0,0)$ & 1 \\
\hline \multicolumn{2}{|l|}{1 S.I. } & \multicolumn{2}{|l|}{1 S.I. } & \multicolumn{2}{|l|}{2 S.I. } & \multicolumn{2}{|c|}{4 S.I. } & 6 S.I. & & 9 S.I. & & $11 \mathrm{~s}$. & \\
\hline
\end{tabular}

Tabela 2.24: Neste caso obtemos a distribuição de multipletos do código genético quebrando o primeiro $\mathfrak{s u}(2)$ para $\mathfrak{o}(2)$ e depois para $\mathfrak{s o}(2)$. Esta distribuição está apresentada na tabela seguinte. 


\begin{tabular}{|c|c|c|c|c|c|}
\hline \multicolumn{2}{|c|}{$\left(A_{1}\right)^{3}$} & \multicolumn{2}{|c|}{$\left(L_{1, z}^{2}\right)$} & \multicolumn{2}{|l|}{$\left(L_{1, z}\right)$} \\
\hline Peso & $d$ & Peso & $d$ & Peso & $d$ \\
\hline \multirow[t]{3}{*}{$(2,1,1)$} & \multirow[t]{3}{*}{12} & \multirow[t]{2}{*}{$( \pm 2,1,1)$} & \multirow[t]{2}{*}{8} & $(+2,1,1)$ & 4 \\
\hline & & & & $(-2,1,1)$ & 4 \\
\hline & & $(0,1,1)$ & 4 & $(0,1,1)$ & 4 \\
\hline$(0,1,1)$ & 4 & $(0,1,1)$ & 4 & $(0,1,1)$ & 4 \\
\hline \multirow[t]{2}{*}{$(1,2,1)$} & \multirow[t]{2}{*}{12} & \multirow[t]{2}{*}{$( \pm 1,2,1)$} & \multirow[t]{2}{*}{12} & $(+1,2,1)$ & 6 \\
\hline & & & & $(-1,2,1)$ & 6 \\
\hline \multirow[t]{4}{*}{$(3,1,0)$} & \multirow[t]{4}{*}{8} & \multirow[t]{2}{*}{$( \pm 3,1,0)$} & \multirow[t]{2}{*}{4} & $(+3,1,0)$ & 2 \\
\hline & & & & $(-3,1,0)$ & 2 \\
\hline & & \multirow[t]{2}{*}{$( \pm 1,1,0)$} & \multirow[t]{2}{*}{4} & $(+1,1,0)$ & 2 \\
\hline & & & & $(-1,1,0)$ & 2 \\
\hline \multirow[t]{2}{*}{$(1,1,0)$} & \multirow[t]{2}{*}{4} & \multirow[t]{2}{*}{$( \pm 1,1,0)$} & \multirow[t]{2}{*}{4} & $(+1,1,0)$ & 2 \\
\hline & & & & $(-1,1,0)$ & 2 \\
\hline \multirow[t]{3}{*}{$(2,2,0)$} & \multirow[t]{3}{*}{9} & \multirow{2}{*}{$( \pm 2,2,0)$} & \multirow[t]{2}{*}{6} & $(+2,2,0)$ & 3 \\
\hline & & & & $(-2,2,0)$ & 3 \\
\hline & & $(0,2,0)$ & 3 & $(0,2,0)$ & 3 \\
\hline \multirow[t]{2}{*}{$(1,0,1)$} & \multirow[t]{2}{*}{4} & \multirow[t]{2}{*}{$( \pm 1,0,1)$} & \multirow[t]{2}{*}{4} & $(+1,0,1)$ & 2 \\
\hline & & & & $(-1,0,1)$ & 2 \\
\hline \multirow[t]{2}{*}{$(2,0,0)$} & \multirow[t]{2}{*}{3} & \multirow[t]{2}{*}{$( \pm 2,0,0)$} & \multirow[t]{2}{*}{3} & $(+2,0,0)$ & 1 \\
\hline & & & & $(0,0,0)$ & 1 \\
\hline$(1,1,0)$ & 4 & $( \pm 1,1,0)$ & 4 & $(+1,1,0)$ & 2 \\
\hline & & & & $(-1,1,0)$ & 2 \\
\hline$(0,2,0)$ & 3 & $(0,2,0)$ & 3 & $(0,2,0)$ & 3 \\
\hline$(0,0,0)$ & 1 & $(0,0,0)$ & 1 & $(0,0,0)$ & 1 \\
\hline 11 & & 15 & & $24 \mathrm{~s}$ & \\
\hline
\end{tabular}


4) $D_{7} \supset B_{3} \oplus B_{3} \supset B_{3} \oplus G_{2} \supset G_{2} \oplus G_{2} \supset G_{2} \oplus A_{1} \supset A_{1} \oplus A_{1} \oplus A_{1} \supset\left(A_{1}\right)_{13} \oplus A_{1}$.

\begin{tabular}{|c|c|c|c|c|c|c|c|c|c|c|c|c|c|}
\hline \multicolumn{2}{|l|}{$D_{7}$} & \multicolumn{2}{|c|}{$B_{3} \oplus B_{3}$} & \multicolumn{2}{|c|}{$B_{3} \oplus G_{2}$} & \multicolumn{2}{|c|}{$G_{2} \oplus G_{2}$} & \multicolumn{2}{|c|}{$G_{2} \oplus A_{1}$} & \multicolumn{2}{|c|}{$\left(A_{1}\right)^{3}$} & \multicolumn{2}{|c|}{$\left(A_{1}\right)^{2}$} \\
\hline Peso & $d$ & Peso & $d$ & Peso & $d$ & Peso & $d$ & Peso & $d$ & Peso & $d$ & Peso & $d$ \\
\hline \multirow[t]{7}{*}{ (0000010) } & \multirow[t]{7}{*}{64} & \multirow[t]{7}{*}{$(001,001)$} & \multirow[t]{7}{*}{64} & \multirow[t]{4}{*}{$(001,01)$} & \multirow[t]{4}{*}{56} & \multirow[t]{3}{*}{$(01,01)$} & \multirow[t]{3}{*}{49} & \multirow[t]{3}{*}{$(01,6)$} & \multirow[t]{3}{*}{49} & \multirow[t]{2}{*}{$(1,1,6)$} & \multirow[t]{2}{*}{28} & $(7,1)$ & 16 \\
\hline & & & & & & & & & & & & $(5,1)$ & 12 \\
\hline & & & & & & & & & & $(0,2,6)$ & 21 & $(6,2)$ & 21 \\
\hline & & & & & & $(00,01)$ & 7 & $(00,6)$ & 7 & $(0,0,6)$ & 7 & $(6,0)$ & 7 \\
\hline & & & & \multirow[t]{3}{*}{$(001,00)$} & \multirow[t]{3}{*}{8} & \multirow[t]{2}{*}{$(01,00)$} & \multirow[t]{2}{*}{7} & \multirow[t]{2}{*}{$(01,0)$} & \multirow[t]{2}{*}{7} & $(1,1,0)$ & 4 & $(1,1)$ & 4 \\
\hline & & & & & & & & & & $(0,2,0)$ & 3 & $(0,2)$ & 3 \\
\hline & & & & & & $(00,00)$ & 1 & $(00,0)$ & 1 & $(0,0,0)$ & 1 & $(0,0)$ & 1 \\
\hline \multicolumn{2}{|l|}{1 S.I. } & \multicolumn{2}{|l|}{1 S.I. } & \multicolumn{2}{|l|}{2 S.I. } & \multicolumn{2}{|c|}{4 S.I. } & \multicolumn{2}{|c|}{4 S.I. } & \multicolumn{2}{|c|}{6 S.I. } & \multicolumn{2}{|c|}{7 S.I. } \\
\hline
\end{tabular}

Tabela 2.25: Neste caso há duas possibilidades de se obter a distribuição de multipletos do código genético:

a) quebrando o primeiro $\mathfrak{s u}(2)$ para $\mathfrak{o}(2)$ e depois para $\mathfrak{s o}(2)$.

b) quebrando o primeiro $\mathfrak{s u}(2)$ para $\mathfrak{o}(2)$ e depois o segundo $\mathfrak{s u}(2)$ para $\mathfrak{s o}(2)$. Ambas as possibilidades estão apresentadas nas duas tabelas seguintes. 


\begin{tabular}{|c|c|c|c|c|c|}
\hline \multicolumn{2}{|c|}{$\left(A_{1}\right)^{2}$} & \multicolumn{2}{|c|}{$\left(L_{1, z}^{2}\right)$} & \multicolumn{2}{|c|}{$\left(L_{1, z}\right)$} \\
\hline Peso & $d$ & Peso & $d$ & Peso & $d$ \\
\hline \multirow[t]{8}{*}{$(7,1)$} & \multirow[t]{8}{*}{16} & \multirow[t]{2}{*}{$( \pm 7,1)$} & \multirow[t]{2}{*}{4} & $(+7,1)$ & 2 \\
\hline & & & & $(-7,1)$ & 2 \\
\hline & & \multirow[t]{2}{*}{$( \pm 5,1)$} & \multirow[t]{2}{*}{4} & $(+5,1)$ & 2 \\
\hline & & & & $(-5,1)$ & 2 \\
\hline & & $( \pm 3,1)$ & 4 & $(+3,1)$ & 2 \\
\hline & & & & $(-3,1)$ & 2 \\
\hline & & \multirow[t]{2}{*}{$( \pm 1,1)$} & \multirow[t]{2}{*}{4} & $(+1,1)$ & 2 \\
\hline & & & & $(-1,1)$ & 2 \\
\hline \multirow[t]{6}{*}{$(5,1)$} & \multirow[t]{6}{*}{12} & \multirow[t]{2}{*}{$( \pm 5,1)$} & \multirow[t]{2}{*}{4} & $(+5,1)$ & 2 \\
\hline & & & & $(-5,1)$ & 2 \\
\hline & & \multirow[t]{2}{*}{$( \pm 3,1)$} & \multirow[t]{2}{*}{4} & $(+3,1)$ & 2 \\
\hline & & & & $(-3,1)$ & 2 \\
\hline & & \multirow[t]{2}{*}{$( \pm 1,1)$} & \multirow[t]{2}{*}{4} & $(+1,1)$ & 2 \\
\hline & & & & $(-1,1)$ & 2 \\
\hline \multirow[t]{7}{*}{$(6,2)$} & \multirow[t]{7}{*}{21} & \multirow[t]{2}{*}{$( \pm 6,2)$} & \multirow[t]{2}{*}{6} & $(+6,2)$ & 3 \\
\hline & & & & $(-6,2)$ & 3 \\
\hline & & \multirow[t]{2}{*}{$( \pm 4,2)$} & \multirow[t]{2}{*}{6} & $(+4,2)$ & 3 \\
\hline & & & & $(-4,2)$ & 3 \\
\hline & & \multirow[t]{2}{*}{$( \pm 2,2)$} & \multirow[t]{2}{*}{6} & $(+2,2)$ & 3 \\
\hline & & & & $(-2,2)$ & 3 \\
\hline & & $(0,2)$ & 3 & $(0,2)$ & 3 \\
\hline \multirow[t]{7}{*}{$(6,0)$} & \multirow[t]{7}{*}{7} & $( \pm 6,0)$ & 2 & $(+6,0)$ & 1 \\
\hline & & & & $(-6,0)$ & 1 \\
\hline & & $( \pm 4,0)$ & 2 & $(+4,0)$ & 1 \\
\hline & & & & $(-4,0)$ & 1 \\
\hline & & $( \pm 2,0)$ & 2 & $(+2,0)$ & 1 \\
\hline & & & & $(-2,0)$ & 1 \\
\hline & & $(0,0)$ & 1 & $(0,0)$ & 1 \\
\hline$(1,1)$ & 4 & $( \pm 1,1)$ & 4 & $(+1,1)$ & 2 \\
\hline & & & & $(-1,1)$ & 2 \\
\hline$(0,2)$ & 3 & $(0,2)$ & 3 & $(0,2)$ & 3 \\
\hline$(0,0)$ & 1 & $(0,0)$ & 1 & $(0,0)$ & 1 \\
\hline $7 \mathrm{~s}$ & & $18 \mathrm{~S} . \mathrm{l}$ & & $32 \mathrm{~s}$. & \\
\hline
\end{tabular}




\begin{tabular}{|c|c|c|c|c|c|}
\hline \multicolumn{2}{|c|}{$\left(A_{1}\right)^{2}$} & \multicolumn{2}{|c|}{$\left(L_{1, z}^{2}\right)$} & \multicolumn{2}{|c|}{$\left(L_{1, z}^{2}, L_{2, z}\right)$} \\
\hline Peso & $d$ & Peso & $d$ & Peso & $d$ \\
\hline \multirow[t]{6}{*}{$(7,1)$} & \multirow[t]{6}{*}{16} & $( \pm 7,1)$ & 4 & $\begin{array}{l}( \pm 7,+1) \\
( \pm 7,-1)\end{array}$ & $\begin{array}{l}2 \\
2\end{array}$ \\
\hline & & $( \pm 5,1)$ & 4 & $( \pm 5,+1)$ & 2 \\
\hline & & $( \pm 3,1)$ & 4 & $\begin{array}{l}( \pm 5,-1) \\
( \pm 3,+1)\end{array}$ & $\begin{array}{l}2 \\
2\end{array}$ \\
\hline & & & & $( \pm 3,-1)$ & 2 \\
\hline & & $( \pm 1,1)$ & 4 & $( \pm 1,+1)$ & 2 \\
\hline & & & & $( \pm 1,-1)$ & 2 \\
\hline \multirow[t]{6}{*}{$(5,1)$} & \multirow[t]{6}{*}{12} & \multirow[t]{2}{*}{$( \pm 5,1)$} & \multirow[t]{2}{*}{4} & $( \pm 5,+1)$ & 2 \\
\hline & & & & $( \pm 5,-1)$ & 2 \\
\hline & & \multirow[t]{2}{*}{$( \pm 3,1)$} & \multirow[t]{2}{*}{4} & $( \pm 3,+1)$ & 2 \\
\hline & & & & $( \pm 3,-1)$ & 2 \\
\hline & & \multirow[t]{2}{*}{$( \pm 1,1)$} & \multirow[t]{2}{*}{4} & $( \pm 1,+1)$ & 2 \\
\hline & & & & $( \pm 1,-1)$ & 2 \\
\hline \multirow[t]{12}{*}{$(6,2)$} & \multirow[t]{12}{*}{21} & \multirow[t]{3}{*}{$( \pm 6,2)$} & \multirow[t]{3}{*}{6} & $( \pm 6,+2)$ & 2 \\
\hline & & & & $( \pm 6,-2)$ & 2 \\
\hline & & & & $( \pm 6,0)$ & 2 \\
\hline & & \multirow[t]{3}{*}{$( \pm 4,2)$} & \multirow[t]{3}{*}{6} & $( \pm 4,+2)$ & 2 \\
\hline & & & & $( \pm 4,-2)$ & 2 \\
\hline & & & & $( \pm 4,0)$ & 2 \\
\hline & & \multirow[t]{3}{*}{$( \pm 2,2)$} & \multirow[t]{3}{*}{6} & $( \pm 2,+2)$ & 2 \\
\hline & & & & $( \pm 2,-2)$ & 2 \\
\hline & & & & $( \pm 2,0)$ & 2 \\
\hline & & \multirow[t]{3}{*}{$(0,2)$} & \multirow[t]{3}{*}{3} & $(0,+2)$ & 1 \\
\hline & & & & $(0,-2)$ & 1 \\
\hline & & & & $(0,0)$ & 1 \\
\hline \multirow[t]{4}{*}{$(6,0)$} & \multirow[t]{4}{*}{7} & $( \pm 6,0)$ & 2 & $( \pm 6,0)$ & 2 \\
\hline & & $( \pm 4,0)$ & 2 & $( \pm 4,0)$ & 2 \\
\hline & & $( \pm 2,0)$ & 2 & $( \pm 2,0)$ & 2 \\
\hline & & $(0,0)$ & 1 & $(0,0)$ & 1 \\
\hline \multirow[t]{2}{*}{$(1,1)$} & \multirow[t]{2}{*}{4} & \multirow[t]{2}{*}{$( \pm 1,1)$} & 4 & $( \pm 1,+1)$ & 2 \\
\hline & & & & $( \pm 1,-1)$ & 2 \\
\hline$(0,2)$ & 3 & $(0,2)$ & 3 & $(0,+2)$ & 1 \\
\hline & & & & $(0,-2)$ & 1 \\
\hline & & & & $(0,0)$ & 1 \\
\hline$(0,0)$ & 1 & $(0,0)$ & 1 & $(0,0)$ & 1 \\
\hline $7 \mathrm{~s}$. & & $18 \mathrm{~s}$. & & 36 S.I. & \\
\hline
\end{tabular}


5) $D_{7} \supset A_{3} \oplus D_{4} \supset A_{3} \oplus A_{1} \oplus C_{2} \supset A_{1} \oplus A_{1} \oplus A_{1} \oplus C_{2} \supset A_{1} \oplus A_{1} \oplus A_{1} \oplus A_{1} \supset\left(A_{1}\right)_{13} \oplus A_{1} \oplus A_{1} \supset$ $\supset\left(A_{1}\right)_{23} \oplus A_{1}$.

\begin{tabular}{|c|c|c|c|c|c|c|c|c|c|c|c|c|c|}
\hline \multicolumn{2}{|l|}{$D_{7}$} & \multicolumn{2}{|c|}{$A_{3} \oplus D_{4}$} & \multicolumn{2}{|c|}{$A_{3} \oplus A_{1} \oplus C_{2}$} & \multicolumn{2}{|c|}{$\left(A_{1}\right)^{3} \oplus C_{2}$} & \multicolumn{2}{|l|}{$\left(A_{1}\right)^{4}$} & \multicolumn{2}{|c|}{$\left(A_{1}\right)^{3}$} & \multicolumn{2}{|c|}{$\left(A_{1}\right)^{2}$} \\
\hline Peso & $d$ & Peso & $d$ & Peso & $d$ & Peso & $d$ & Peso & $d$ & Peso & $d$ & Peso & $d$ \\
\hline \multirow[t]{8}{*}{ (0000010) } & \multirow[t]{8}{*}{64} & \multirow[t]{4}{*}{$(001,0010)$} & \multirow[t]{4}{*}{32} & \multirow[t]{2}{*}{$(001,0,01)$} & \multirow[t]{2}{*}{20} & \multirow[t]{2}{*}{$(1,1,0,01)$} & \multirow[t]{2}{*}{20} & \multirow[t]{2}{*}{$(1,1,0,4)$} & \multirow[t]{2}{*}{20} & \multirow[t]{2}{*}{$(1,1,4)$} & \multirow[t]{2}{*}{20} & $(5,1)$ & 12 \\
\hline & & & & & & & & & & & & $(3,1)$ & 8 \\
\hline & & & & \multirow[t]{2}{*}{$(001,2,00)$} & \multirow[t]{2}{*}{12} & \multirow[t]{2}{*}{$(1,1,2,00)$} & \multirow[t]{2}{*}{12} & \multirow[t]{2}{*}{$(1,1,2,0)$} & \multirow[t]{2}{*}{12} & $(3,1,0)$ & 8 & $(1,3)$ & 8 \\
\hline & & & & & & & & & & $(1,1,0)$ & 4 & $(1,1)$ & 4 \\
\hline & & \multirow[t]{4}{*}{$(100,0001)$} & \multirow[t]{4}{*}{32} & \multirow[t]{4}{*}{$(100,1,10)$} & \multirow[t]{4}{*}{32} & \multirow[t]{4}{*}{$(1,1,1,10)$} & \multirow[t]{4}{*}{32} & \multirow[t]{4}{*}{$(1,1,1,3)$} & \multirow[t]{4}{*}{32} & \multirow[t]{2}{*}{$(2,1,3)$} & \multirow[t]{2}{*}{24} & $(4,2)$ & 15 \\
\hline & & & & & & & & & & & & $(2,2)$ & 9 \\
\hline & & & & & & & & & & \multirow[t]{2}{*}{$(0,1,3)$} & \multirow[t]{2}{*}{8} & $(4,0)$ & 5 \\
\hline & & & & & & & & & & & & $(2,0)$ & 3 \\
\hline 1 S.I. & & 2 S.I. & & 3 S.I. & & 3 S.I. & & 3 S.I. & & $5 \mathrm{~S} . \mathrm{I}$ & & $8 \mathrm{~s}$ & \\
\hline
\end{tabular}

Tabela 2.26: Neste caso há duas possibilidades de se obter a distribuição de multipletos do código genético:

a) quebrando o primeiro $\mathfrak{s u}(2)$ para $\mathfrak{o}(2)$ e depois para $\mathfrak{s o}(2)$.

b) quebrando o primeiro $\mathfrak{s u}(2)$ para $\mathfrak{o}(2)$ e depois o segundo $\mathfrak{s u}(2)$ para $\mathfrak{s o}(2)$. Ambas as possibilidades estão apresentadas nas duas tabelas seguintes. 


\begin{tabular}{|c|c|c|c|c|c|}
\hline \multicolumn{2}{|c|}{$\left(A_{1}\right)^{2}$} & \multicolumn{2}{|c|}{$\left(L_{1, z}^{2}\right)$} & \multicolumn{2}{|c|}{$\left(L_{1, z}\right)$} \\
\hline Peso & $d$ & Peso & $d$ & Peso & $d$ \\
\hline \multirow[t]{5}{*}{$(5,1)$} & \multirow[t]{5}{*}{12} & $( \pm 5,1)$ & 4 & $\begin{array}{l}(+5,1) \\
(-5,1)\end{array}$ & 2 \\
\hline & & \multirow{2}{*}{$( \pm 3,1)$} & \multirow{2}{*}{4} & $(+3,1)$ & 2 \\
\hline & & & & $(-3,1)$ & 2 \\
\hline & & \multirow[t]{2}{*}{$( \pm 1,1)$} & \multirow[t]{2}{*}{4} & $(+1,1)$ & 2 \\
\hline & & & & $(-1,1)$ & 2 \\
\hline \multirow[t]{4}{*}{$(3,1)$} & \multirow[t]{4}{*}{8} & \multirow[t]{2}{*}{$( \pm 3,1)$} & \multirow[t]{2}{*}{4} & $(+3,1)$ & 2 \\
\hline & & & & $(-3,1)$ & 2 \\
\hline & & \multirow[t]{2}{*}{$( \pm 1,1)$} & \multirow[t]{2}{*}{4} & $(+1,1)$ & 2 \\
\hline & & & & $(-1,1)$ & 2 \\
\hline \multirow[t]{2}{*}{$(1,3)$} & \multirow[t]{2}{*}{8} & \multirow[t]{2}{*}{$( \pm 1,3)$} & \multirow[t]{2}{*}{8} & $(+1,3)$ & 4 \\
\hline & & & & $(-1,3)$ & 4 \\
\hline \multirow[t]{2}{*}{$(1,1)$} & \multirow[t]{2}{*}{4} & \multirow[t]{2}{*}{$( \pm 1,1)$} & \multirow[t]{2}{*}{4} & $(+1,1)$ & 2 \\
\hline & & & & $(-1,1)$ & 2 \\
\hline \multirow[t]{5}{*}{$(4,2)$} & \multirow[t]{5}{*}{15} & \multirow[t]{2}{*}{$( \pm 4,2)$} & \multirow[t]{2}{*}{6} & $(+4,2)$ & 3 \\
\hline & & & & $(-4,2)$ & 3 \\
\hline & & \multirow[t]{2}{*}{$( \pm 2,2)$} & 6 & $(+2,2)$ & 3 \\
\hline & & & & $(-2,2)$ & 3 \\
\hline & & $(0,2)$ & 3 & $(0,2)$ & 3 \\
\hline$(2,2)$ & 9 & $( \pm 2,2)$ & 6 & $(+2,2)$ & 3 \\
\hline & & & & $(-2,2)$ & 3 \\
\hline & & $(0,2)$ & 3 & $(0,2)$ & 3 \\
\hline$(4,0)$ & 5 & $( \pm 4,0)$ & 2 & $(+4,0)$ & 1 \\
\hline & & & & $(-4,0)$ & 1 \\
\hline & & $( \pm 2,0)$ & 2 & $(+2,0)$ & 1 \\
\hline & & & & $(-2,0)$ & 1 \\
\hline & & $(0,0)$ & 1 & $(0,0)$ & 1 \\
\hline$(2,0)$ & 3 & $( \pm 2,0)$ & 2 & $(+2,0)$ & 1 \\
\hline & & & & $(-2,0)$ & 1 \\
\hline & & $(0,0)$ & 1 & $(0,0)$ & 1 \\
\hline 8 & & 18 & & $32 \mathrm{~s}$ & \\
\hline
\end{tabular}




\begin{tabular}{|c|c|c|c|c|c|}
\hline \multicolumn{2}{|c|}{$\left(A_{1}\right)^{2}$} & \multicolumn{2}{|c|}{$\left(L_{1, z}^{2}\right)$} & \multicolumn{2}{|c|}{$\left(L_{1, z}^{2}, L_{2, z}\right)$} \\
\hline Peso & $d$ & Peso & $d$ & Peso & $d$ \\
\hline \multirow[t]{6}{*}{$(5,1)$} & \multirow[t]{6}{*}{12} & \multirow[t]{2}{*}{$( \pm 5,1)$} & \multirow[t]{2}{*}{4} & $( \pm 5,+1)$ & 2 \\
\hline & & & & $( \pm 5,-1)$ & 2 \\
\hline & & \multirow[t]{2}{*}{$( \pm 3,1)$} & \multirow[t]{2}{*}{4} & $( \pm 3,+1)$ & 2 \\
\hline & & & & $( \pm 3,-1)$ & 2 \\
\hline & & \multirow[t]{2}{*}{$( \pm 1,1)$} & \multirow[t]{2}{*}{4} & $( \pm 1,+1)$ & 2 \\
\hline & & & & $( \pm 1,-1)$ & 2 \\
\hline \multirow[t]{4}{*}{$(3,1)$} & \multirow[t]{4}{*}{8} & \multirow[t]{2}{*}{$( \pm 3,1)$} & \multirow[t]{2}{*}{4} & $( \pm 3,+1)$ & 2 \\
\hline & & & & $( \pm 3,-1)$ & 2 \\
\hline & & \multirow[t]{2}{*}{$( \pm 1,1)$} & \multirow[t]{2}{*}{4} & $( \pm 1,+1)$ & 2 \\
\hline & & & & $( \pm 1,-1)$ & 2 \\
\hline \multirow[t]{4}{*}{$(1,3)$} & \multirow[t]{4}{*}{8} & \multirow[t]{4}{*}{$( \pm 1,3)$} & \multirow[t]{4}{*}{8} & $( \pm 1,+3)$ & 2 \\
\hline & & & & $( \pm 1,-3)$ & 2 \\
\hline & & & & $( \pm 1,+1)$ & 2 \\
\hline & & & & $( \pm 1,-1)$ & 2 \\
\hline \multirow[t]{2}{*}{$(1,1)$} & \multirow[t]{2}{*}{4} & \multirow[t]{2}{*}{$( \pm 1,1)$} & \multirow[t]{2}{*}{4} & $( \pm 1,+1)$ & 2 \\
\hline & & & & $( \pm 1,-1)$ & 2 \\
\hline \multirow[t]{9}{*}{$(4,2)$} & \multirow[t]{9}{*}{15} & $( \pm 4,2)$ & 6 & $( \pm 4,+2)$ & 2 \\
\hline & & & & $( \pm 4,-2)$ & 2 \\
\hline & & & & $( \pm 4,0)$ & 2 \\
\hline & & $( \pm 2,2)$ & 6 & $( \pm 2,+2)$ & 2 \\
\hline & & & & $( \pm 2,-2)$ & 2 \\
\hline & & & & $( \pm 2,0)$ & 2 \\
\hline & & $(0,2)$ & 3 & $(0,+2)$ & 1 \\
\hline & & & & $(0,-2)$ & 1 \\
\hline & & & & $(0,0)$ & 1 \\
\hline$(2,2)$ & 9 & $( \pm 2,2)$ & 6 & $( \pm 2,+2)$ & 2 \\
\hline & & & & $( \pm 2,-2)$ & 2 \\
\hline & & & & $( \pm 2,0)$ & 2 \\
\hline & & $(0,2)$ & 3 & $(0,+2)$ & 1 \\
\hline & & & & $(0,-2)$ & 1 \\
\hline & & & & $(0,0)$ & 1 \\
\hline$(4,0)$ & 5 & $( \pm 4,0)$ & 2 & $( \pm 4,0)$ & 2 \\
\hline & & $( \pm 2,0)$ & 2 & $( \pm 2,0)$ & 2 \\
\hline & & $(0,0)$ & 1 & $(0,0)$ & 1 \\
\hline$(2,0)$ & 3 & $( \pm 2,0)$ & 2 & $( \pm 2,0)$ & 2 \\
\hline & & $(0,0)$ & 1 & $(0,0)$ & 1 \\
\hline $8 \mathrm{~s}$ & & $17 \mathrm{~s}$. & & 36 S.I. & \\
\hline
\end{tabular}





\section{Cadeias Sobreviventes de $B_{6}$}

Este apêndice contém as cadeias sobreviventes de $B_{6}=\mathfrak{s o}(13)$. A primeira tabela lista as cadeias e indica a págine em que se encontram. 


\begin{tabular}{|c|c|c|}
\hline Número & Página & Cadeia \\
\hline 1 & 85 & $B_{6} \supset D_{6} \supset A_{1} \oplus C_{3} \supset A_{1} \oplus A_{1}$ \\
\hline 2 & 85 & $B_{6} \supset D_{6} \supset A_{1} \oplus C_{3} \supset A_{1} \oplus A_{1} \oplus A_{1}$ \\
\hline 3 & 86 & $\begin{aligned} B_{6} & \supset D_{6} \supset A_{1} \oplus A_{1} \oplus D_{4} \supset A_{1} \oplus A_{1} \oplus A_{1} \oplus C_{2} \supset \\
& \supset A_{1} \oplus A_{1} \oplus A_{1} \oplus A_{1} \oplus A_{1} \supset\left(A_{1}\right)_{14} \oplus A_{1} \oplus A_{1} \oplus A_{1}\end{aligned}$ \\
\hline 4 & 86 & $\begin{aligned} B_{6} & \supset D_{6} \supset A_{1} \oplus C_{3} \supset A_{1} \oplus A_{1} \oplus C_{2} \supset \\
& \supset A_{1} \oplus A_{1} \oplus A_{1} \oplus A_{1} \supset\left(A_{1}\right)_{23} \oplus A_{1} \oplus A_{1}\end{aligned}$ \\
\hline 5 & 87 & $\begin{aligned} B_{6} & \supset D_{6} \supset A_{1} \oplus A_{1} \oplus D_{4} \supset A_{1} \oplus A_{1} \oplus A_{1} \oplus C_{2} \supset \\
& \supset A_{1} \oplus A_{1} \oplus A_{1} \oplus A_{1} \supset\left(A_{1}\right)_{14} \oplus A_{1} \oplus A_{1}\end{aligned}$ \\
\hline 6 & 87 & $\begin{aligned} B_{6} & \supset C_{2} \oplus D_{4} \supset C_{2} \oplus A_{1} \oplus C_{2} \supset A_{1} \oplus A_{1} \oplus A_{1} \oplus A_{1} \supset \\
& \supset\left(A_{1}\right)_{13} \oplus A_{1} \oplus A_{1}\end{aligned}$ \\
\hline 7 & 88 & $\begin{aligned} B_{6} & \supset C_{2} \oplus D_{4} \supset C_{2} \oplus A_{1} \oplus C_{2} \supset A_{1} \oplus A_{1} \oplus A_{1} \oplus A_{1} \supset \\
& \supset\left(A_{1}\right)_{34} \oplus A_{1} \oplus A_{1}\end{aligned}$ \\
\hline 8 & 88 & $\begin{aligned} B_{6} & \supset D_{6} \supset A_{1} \oplus A_{1} \oplus D_{4} \supset A_{1} \oplus A_{1} \oplus A_{1} \oplus C_{2} \supset \\
& \supset A_{1} \oplus A_{1} \oplus A_{1} \oplus A_{1} \oplus A_{1} \supset\left(A_{1}\right)_{14} \oplus A_{1} \oplus A_{1} \oplus A_{1} \supset \\
& \supset\left(A_{1}\right)_{24} \oplus A_{1} \oplus A_{1}\end{aligned}$ \\
\hline 9 & 89 & $\begin{aligned} B_{6} & \supset D_{6} \supset A_{1} \oplus C_{3} \supset A_{1} \oplus A_{1} \oplus C_{2} \supset \\
& \supset A_{1} \oplus A_{1} \oplus A_{1} \supset\left(A_{1}\right)_{23} \oplus A_{1}\end{aligned}$ \\
\hline 10 & 89 & $\begin{aligned} B_{6} & \supset C_{2} \oplus D_{4} \supset C_{2} \oplus A_{1} \oplus C_{2} \supset A_{1} \oplus A_{1} \oplus A_{1} \supset \\
& \supset\left(A_{1}\right)_{13} \oplus A_{1}\end{aligned}$ \\
\hline 11 & 90 & $\begin{aligned} B_{6} & \supset D_{6} \supset A_{1} \oplus C_{3} \supset A_{1} \oplus A_{1} \oplus C_{2} \supset \\
& \supset A_{1} \oplus A_{1} \oplus A_{1} \oplus A_{1} \supset\left(A_{1}\right)_{23} \oplus A_{1} \oplus A_{1} \supset \\
& \supset\left(A_{1}\right)_{23} \oplus A_{1}\end{aligned}$ \\
\hline 12 & 91 & $\begin{aligned} B_{6} & \supset C_{2} \oplus D_{4} \supset C_{2} \oplus A_{1} \oplus C_{2} \supset A_{1} \oplus A_{1} \oplus A_{1} \oplus A_{1} \supset \\
& \supset\left(A_{1}\right)_{12} \oplus A_{1} \oplus A_{1} \supset\left(A_{1}\right)_{23} \oplus A_{1}\end{aligned}$ \\
\hline 13 & 91 & $\begin{aligned} B_{6} & \supset A_{1} \oplus A_{1} \oplus B_{4} \supset A_{1} \oplus A_{1} \oplus A_{1} \oplus A_{1} \supset \\
& \supset\left(A_{1}\right)_{13} \oplus A_{1} \oplus A_{1} \supset\left(A_{1}\right)_{23} \oplus A_{1}\end{aligned}$ \\
\hline
\end{tabular}


1. $B_{6} \supset D_{6} \supset A_{1} \oplus C_{3} \supset A_{1} \oplus A_{1}$.

\begin{tabular}{|c|c|c|c|c|c|c|c|}
\hline \multicolumn{2}{|l|}{$B_{6}$} & \multicolumn{2}{|l|}{$D_{6}$} & \multicolumn{2}{|c|}{$A_{1} \oplus C_{3}$} & \multicolumn{2}{|c|}{$\left(A_{1}\right)^{2}$} \\
\hline Peso & $d$ & Peso & $d$ & Peso & $d$ & Peso & $d$ \\
\hline \multirow[t]{6}{*}{ (000001) } & \multirow[t]{6}{*}{64} & \multirow[t]{3}{*}{$(000010)$} & \multirow[t]{3}{*}{32} & \multirow[t]{2}{*}{$(0,001)$} & \multirow[t]{2}{*}{14} & $(0,9)$ & 10 \\
\hline & & & & & & $(0,3)$ & 4 \\
\hline & & & & $(2,100)$ & 18 & $(2,5)$ & 18 \\
\hline & & \multirow[t]{3}{*}{$(000001)$} & \multirow[t]{3}{*}{32} & \multirow[t]{2}{*}{$(0,010)$} & \multirow[t]{2}{*}{28} & $(1,8)$ & 18 \\
\hline & & & & & & $(1,4)$ & 10 \\
\hline & & & & $(3,000)$ & 4 & $(3,0)$ & 4 \\
\hline \multicolumn{2}{|c|}{1 S.I. } & \multicolumn{2}{|l|}{2 S.I. } & \multicolumn{2}{|c|}{4 S.I. } & \multicolumn{2}{|c|}{6 S.I. } \\
\hline
\end{tabular}

2. $B_{6} \supset D_{6} \supset A_{1} \oplus C_{3} \supset A_{1} \oplus A_{1} \oplus A_{1}$.

\begin{tabular}{|c|c|c|c|c|c|c|c|}
\hline \multicolumn{2}{|l|}{$B_{6}$} & \multicolumn{2}{|l|}{$D_{6}$} & \multicolumn{2}{|c|}{$A_{1} \oplus C_{3}$} & \multicolumn{2}{|c|}{$\left(A_{1}\right)^{3}$} \\
\hline Peso & $d$ & Peso & $d$ & Peso & $d$ & Peso & $d$ \\
\hline \multirow[t]{6}{*}{ (000001) } & \multirow[t]{6}{*}{64} & \multirow[t]{3}{*}{ (000010) } & \multirow[t]{3}{*}{32} & \multirow[t]{2}{*}{$(0,001)$} & \multirow[t]{2}{*}{14} & $(0,1,4)$ & 10 \\
\hline & & & & & & $(0,3,0)$ & 4 \\
\hline & & & & $(2,100)$ & 18 & $(2,1,2)$ & 18 \\
\hline & & \multirow[t]{3}{*}{$(000001)$} & \multirow[t]{3}{*}{32} & \multirow[t]{2}{*}{$(0,010)$} & \multirow[t]{2}{*}{28} & $(1,0,4)$ & 18 \\
\hline & & & & & & $(1,2,2)$ & 10 \\
\hline & & & & $(3,000)$ & 4 & $(3,0,0)$ & 4 \\
\hline \multicolumn{2}{|l|}{1 S.I. } & \multicolumn{2}{|l|}{2 S.I. } & \multicolumn{2}{|c|}{4 S.I. } & \multicolumn{2}{|c|}{6 S.I } \\
\hline
\end{tabular}


3. $B_{6} \supset D_{6} \supset A_{1} \oplus A_{1} \oplus D_{4} \supset A_{1} \oplus A_{1} \oplus A_{1} \oplus C_{2} \supset A_{1} \oplus A_{1} \oplus A_{1} \oplus A_{1} \oplus A_{1} \supset\left(A_{1}\right)_{14} \oplus A_{1} \oplus A_{1} \oplus A_{1}$.

\begin{tabular}{|c|c|c|c|c|c|c|c|c|c|c|c|}
\hline \multicolumn{2}{|l|}{$B_{6}$} & \multicolumn{2}{|l|}{$D_{6}$} & \multicolumn{2}{|c|}{$\left(A_{1}\right)^{2} \oplus D_{4}$} & \multicolumn{2}{|c|}{$\left(A_{1}\right)^{3} \oplus C_{2}$} & \multicolumn{2}{|l|}{$\left(A_{1}\right)^{5}$} & \multicolumn{2}{|l|}{$\left(A_{1}\right)^{4}$} \\
\hline Peso & $d$ & Peso & $d$ & Peso & $d$ & Peso & $d$ & Peso & $d$ & Peso & $d$ \\
\hline \multirow[t]{12}{*}{$(000001)$} & \multirow[t]{12}{*}{64} & \multirow[t]{6}{*}{$(000010)$} & \multirow[t]{6}{*}{32} & \multirow[t]{3}{*}{$(0,1,0010)$} & \multirow[t]{3}{*}{16} & \multirow[t]{2}{*}{$(0,1,0,01)$} & \multirow[t]{2}{*}{10} & $(0,1,0,1,1)$ & 8 & $(1,1,0,1)$ & 8 \\
\hline & & & & & & & & $(0,1,0,0,0)$ & 2 & $(0,1,0,0)$ & 2 \\
\hline & & & & & & $(0,1,2,00)$ & 6 & $(0,1,2,0,0)$ & 6 & $(0,1,2,0)$ & 6 \\
\hline & & & & \multirow[t]{3}{*}{$(1,0,0001)$} & \multirow[t]{3}{*}{16} & \multirow[t]{3}{*}{$(1,0,1,10)$} & \multirow[t]{3}{*}{16} & \multirow[t]{2}{*}{$(1,0,1,1,0)$} & \multirow[t]{2}{*}{8} & $(2,0,1,0)$ & 6 \\
\hline & & & & & & & & & & $(0,0,1,0)$ & 2 \\
\hline & & & & & & & & $(1,0,1,0,1)$ & 8 & $(1,0,1,1)$ & 8 \\
\hline & & \multirow[t]{6}{*}{$(000001)$} & \multirow[t]{6}{*}{32} & \multirow[t]{2}{*}{$(0,1,0001)$} & \multirow[t]{2}{*}{16} & \multirow[t]{2}{*}{$(0,1,1,10)$} & \multirow[t]{2}{*}{16} & $(0,1,1,1,0)$ & 8 & $(1,1,1,0)$ & 8 \\
\hline & & & & & & & & $(0,1,1,0,1)$ & 8 & $(0,1,1,1)$ & 8 \\
\hline & & & & \multirow[t]{4}{*}{$(1,0,0010)$} & \multirow[t]{4}{*}{16} & \multirow[t]{3}{*}{$(1,0,0,01)$} & \multirow[t]{3}{*}{10} & \multirow[t]{2}{*}{$(1,0,0,1,1)$} & \multirow[t]{2}{*}{8} & $(2,0,0,1)$ & 6 \\
\hline & & & & & & & & & & $(0,0,0,1)$ & 2 \\
\hline & & & & & & & & $(1,0,0,0,0)$ & 2 & $(1,0,0,0)$ & 2 \\
\hline & & & & & & $(1,0,2,00)$ & 6 & $(1,0,2,0,0)$ & 6 & $(1,0,2,0)$ & 6 \\
\hline \multicolumn{2}{|c|}{1 S.I. } & \multicolumn{2}{|l|}{2 S.I. } & \multicolumn{2}{|l|}{4 S.I. } & \multicolumn{2}{|l|}{6 S.I. } & 10 S.I. & & 12 S.I. & \\
\hline
\end{tabular}

4. $B_{6} \supset D_{6} \supset A_{1} \oplus C_{3} \supset A_{1} \oplus A_{1} \oplus C_{2} \supset A_{1} \oplus A_{1} \oplus A_{1} \oplus A_{1} \supset\left(A_{1}\right)_{23} \oplus A_{1} \oplus A_{1}$.

\begin{tabular}{|c|c|c|c|c|c|c|c|c|c|c|c|}
\hline \multicolumn{2}{|l|}{$B_{6}$} & \multicolumn{2}{|l|}{$D_{6}$} & \multicolumn{2}{|c|}{$A_{1} \oplus C_{3}$} & \multicolumn{2}{|c|}{$\left(A_{1}\right)^{2} \oplus C_{2}$} & \multicolumn{2}{|l|}{$\left(A_{1}\right)^{4}$} & \multicolumn{2}{|c|}{$\left(A_{1}\right)^{3}$} \\
\hline Peso & $d$ & Peso & $d$ & Peso & $d$ & Peso & $d$ & Peso & $d$ & Peso & $d$ \\
\hline \multirow[t]{15}{*}{ (000001) } & \multirow[t]{15}{*}{64} & \multirow[t]{8}{*}{ (000010) } & \multirow[t]{8}{*}{32} & \multirow[t]{5}{*}{$(0,001)$} & \multirow[t]{5}{*}{14} & \multirow[t]{3}{*}{$(0,1,01)$} & \multirow[t]{3}{*}{10} & \multirow[t]{2}{*}{$(0,1,1,1)$} & \multirow[t]{2}{*}{8} & $(2,0,1)$ & 6 \\
\hline & & & & & & & & & & $(0,0,1)$ & 2 \\
\hline & & & & & & & & $(0,1,0,0)$ & 2 & $(1,0,0)$ & 2 \\
\hline & & & & & & \multirow[t]{2}{*}{$(0,0,10)$} & \multirow[t]{2}{*}{4} & $(0,0,1,0)$ & 2 & $(1,0,0)$ & 2 \\
\hline & & & & & & & & $(0,0,0,1)$ & 2 & $(0,0,1)$ & 2 \\
\hline & & & & \multirow[t]{3}{*}{$(2,100)$} & \multirow[t]{3}{*}{18} & \multirow[t]{2}{*}{$(2,0,10)$} & \multirow[t]{2}{*}{12} & $(2,0,1,0)$ & 6 & $(1,2,0)$ & 6 \\
\hline & & & & & & & & $(2,0,0,1)$ & 6 & $(0,2,1)$ & 6 \\
\hline & & & & & & $(2,0,00)$ & 6 & $(2,1,0,0)$ & 6 & $(1,2,0)$ & 6 \\
\hline & & \multirow[t]{7}{*}{$(000001)$} & \multirow[t]{7}{*}{32} & \multirow[t]{6}{*}{$(0,010)$} & \multirow[t]{6}{*}{28} & \multirow[t]{2}{*}{$(1,0,01)$} & \multirow[t]{2}{*}{10} & $(1,0,1,1)$ & 8 & $(1,1,1)$ & 8 \\
\hline & & & & & & & & $(1,0,0,0)$ & 2 & $(0,1,0)$ & 2 \\
\hline & & & & & & \multirow[t]{3}{*}{$(1,1,10)$} & \multirow[t]{3}{*}{16} & \multirow[t]{2}{*}{$(1,1,1,0)$} & \multirow[t]{2}{*}{8} & $(2,1,0)$ & 6 \\
\hline & & & & & & & & & & $(0,1,0)$ & 2 \\
\hline & & & & & & & & $(1,1,0,1)$ & 8 & $(1,1,1)$ & 8 \\
\hline & & & & & & $(1,0,00)$ & 2 & $(1,0,0,0)$ & 2 & $(0,1,0)$ & 2 \\
\hline & & & & $(3,000)$ & 4 & $(3,0,00)$ & 4 & $(3,0,0,0)$ & 4 & $(0,3,0)$ & 4 \\
\hline \multicolumn{2}{|l|}{1 S.I. } & \multicolumn{2}{|c|}{2 S.I. } & 4 S.I & & 8 S.I & & 13 S.I. & & $15 \mathrm{~s}$. & \\
\hline
\end{tabular}


5. $B_{6} \supset D_{6} \supset A_{1} \oplus A_{1} \oplus D_{4} \supset A_{1} \oplus A_{1} \oplus A_{1} \oplus C_{2} \supset A_{1} \oplus A_{1} \oplus A_{1} \oplus A_{1} \supset\left(A_{1}\right)_{14} \oplus A_{1} \oplus A_{1}$.

\begin{tabular}{|c|c|c|c|c|c|c|c|c|c|c|c|}
\hline \multicolumn{2}{|l|}{$B_{6}$} & \multicolumn{2}{|l|}{$D_{6}$} & \multicolumn{2}{|c|}{$\left(A_{1}\right)^{2} \oplus D_{4}$} & \multicolumn{2}{|c|}{$\left(A_{1}\right)^{3} \oplus C_{2}$} & \multicolumn{2}{|c|}{$\left(A_{1}\right)^{4}$} & \multicolumn{2}{|c|}{$\left(A_{1}\right)^{3}$} \\
\hline Peso & $d$ & Peso & $d$ & Peso & $d$ & Peso & $d$ & Peso & $d$ & Peso & $d$ \\
\hline \multirow[t]{8}{*}{ (000001) } & \multirow[t]{8}{*}{64} & \multirow[t]{4}{*}{ (000010) } & \multirow[t]{4}{*}{32} & \multirow[t]{2}{*}{$(0,1,0010)$} & \multirow[t]{2}{*}{16} & $(0,1,0,01)$ & 10 & $(0,1,0,4)$ & 10 & $(4,0,1)$ & 10 \\
\hline & & & & & & $(0,1,2,00)$ & 6 & $(0,1,2,0)$ & 6 & $(0,1,2)$ & 6 \\
\hline & & & & \multirow[t]{2}{*}{$(1,0,0001)$} & \multirow[t]{2}{*}{16} & \multirow[t]{2}{*}{$(1,0,1,10)$} & \multirow[t]{2}{*}{16} & \multirow[t]{2}{*}{$(1,0,1,3)$} & \multirow[t]{2}{*}{16} & $(4,0,1)$ & 10 \\
\hline & & & & & & & & & & $(2,0,1)$ & 6 \\
\hline & & \multirow[t]{4}{*}{ (000001) } & \multirow[t]{4}{*}{32} & $(0,1,0001)$ & 16 & $(0,1,1,10)$ & 16 & $(0,1,1,3)$ & 16 & $(3,1,1)$ & 16 \\
\hline & & & & \multirow[t]{3}{*}{$(1,0,0010)$} & \multirow[t]{3}{*}{16} & \multirow[t]{2}{*}{$(1,0,0,01)$} & \multirow[t]{2}{*}{10} & \multirow[t]{2}{*}{$(1,0,0,4)$} & \multirow[t]{2}{*}{10} & $(5,0,0)$ & 6 \\
\hline & & & & & & & & & & $(3,0,0)$ & 4 \\
\hline & & & & & & $(1,0,2,00)$ & 6 & $(1,0,2,0)$ & 6 & $(1,0,2)$ & 6 \\
\hline \multicolumn{2}{|l|}{1 S.I. } & \multicolumn{2}{|l|}{2 S.I. } & \multicolumn{2}{|l|}{4 S.I. } & \multicolumn{2}{|l|}{6 S.I. } & \multicolumn{2}{|l|}{6 S.I. } & \multicolumn{2}{|l|}{8 S.I. } \\
\hline
\end{tabular}

6. $B_{6} \supset C_{2} \oplus D_{4} \supset C_{2} \oplus A_{1} \oplus C_{2} \supset A_{1} \oplus A_{1} \oplus A_{1} \oplus A_{1} \supset\left(A_{1}\right)_{13} \oplus A_{1} \oplus A_{1}$.

\begin{tabular}{|c|c|c|c|c|c|c|c|c|c|}
\hline \multicolumn{2}{|l|}{$B_{6}$} & \multicolumn{2}{|c|}{$C_{2} \oplus D_{4}$} & \multicolumn{2}{|c|}{$C_{2} \oplus A_{1} \oplus C_{2}$} & \multicolumn{2}{|c|}{$\left(A_{1}\right)^{4}$} & \multicolumn{2}{|c|}{$\left(A_{1}\right)^{3}$} \\
\hline Peso & $d$ & Peso & $d$ & Peso & $d$ & Peso & $d$ & Peso & $d$ \\
\hline \multirow[t]{7}{*}{$(000001)$} & \multirow[t]{7}{*}{64} & \multirow[t]{4}{*}{$(10,0010)$} & \multirow[t]{4}{*}{32} & \multirow[t]{3}{*}{$(10,0,01)$} & \multirow[t]{3}{*}{20} & \multirow[t]{2}{*}{$(3,0,1,1)$} & \multirow[t]{2}{*}{16} & $(4,0,1)$ & 10 \\
\hline & & & & & & & & $(2,0,1)$ & 6 \\
\hline & & & & & & $(3,0,0,0)$ & 4 & $(3,0,0)$ & 4 \\
\hline & & & & $(10,2,00)$ & 12 & $(3,2,0,0)$ & 12 & $(3,2,0)$ & 12 \\
\hline & & \multirow[t]{3}{*}{$(10,0001)$} & \multirow[t]{3}{*}{32} & \multirow[t]{3}{*}{$(10,1,10)$} & \multirow[t]{3}{*}{32} & \multirow[t]{2}{*}{$(3,1,1,0)$} & \multirow[t]{2}{*}{16} & $(4,1,0)$ & 10 \\
\hline & & & & & & & & $(2,1,0)$ & 6 \\
\hline & & & & & & $(3,1,0,1)$ & 16 & $(3,1,1)$ & 16 \\
\hline \multicolumn{2}{|l|}{1 S.I. } & \multicolumn{2}{|l|}{2 S.I. } & \multicolumn{2}{|l|}{3 S.I. } & \multicolumn{2}{|l|}{5 S.I. } & \multicolumn{2}{|c|}{7 S.I. } \\
\hline
\end{tabular}


7. $B_{6} \supset C_{2} \oplus D_{4} \supset C_{2} \oplus A_{1} \oplus C_{2} \supset A_{1} \oplus A_{1} \oplus A_{1} \oplus A_{1} \supset\left(A_{1}\right)_{34} \oplus A_{1} \oplus A_{1}$.

\begin{tabular}{|c|c|c|c|c|c|c|c|c|c|}
\hline \multicolumn{2}{|l|}{$B_{6}$} & \multicolumn{2}{|c|}{$C_{2} \oplus D_{4}$} & \multicolumn{2}{|c|}{$C_{2} \oplus A_{1} \oplus C_{2}$} & \multicolumn{2}{|c|}{$\left(A_{1}\right)^{4}$} & \multicolumn{2}{|c|}{$\left(A_{1}\right)^{3}$} \\
\hline Peso & $d$ & Peso & $d$ & Peso & $d$ & Peso & $d$ & Peso & $d$ \\
\hline \multirow[t]{6}{*}{$(000001)$} & \multirow[t]{6}{*}{64} & \multirow[t]{4}{*}{$(10,0010)$} & \multirow[t]{4}{*}{32} & \multirow[t]{3}{*}{$(10,0,01)$} & \multirow[t]{3}{*}{20} & \multirow[t]{2}{*}{$(3,0,1,1)$} & \multirow[t]{2}{*}{16} & $(2,3,0)$ & 12 \\
\hline & & & & & & & & $(0,3,0)$ & 4 \\
\hline & & & & & & $(3,0,0,0)$ & 4 & $(0,3,0)$ & 4 \\
\hline & & & & $(10,2,00)$ & 12 & $(3,2,0,0)$ & 12 & $(0,2,3)$ & 12 \\
\hline & & \multirow[t]{2}{*}{$(10,0001)$} & \multirow[t]{2}{*}{32} & \multirow[t]{2}{*}{$(10,1,10)$} & \multirow[t]{2}{*}{32} & $(3,1,1,0)$ & 16 & $(1,3,1)$ & 16 \\
\hline & & & & & & $(3,1,0,1)$ & 16 & $(1,3,1)$ & 16 \\
\hline \multicolumn{2}{|l|}{1 S.I. } & \multicolumn{2}{|l|}{2 S.I. } & \multicolumn{2}{|l|}{3 S.I. } & \multicolumn{2}{|l|}{5 S.I. } & \multicolumn{2}{|c|}{6 S.I. } \\
\hline
\end{tabular}

8. $B_{6} \supset D_{6} \supset A_{1} \oplus A_{1} \oplus D_{4} \supset A_{1} \oplus A_{1} \oplus A_{1} \oplus C_{2} \supset A_{1} \oplus A_{1} \oplus A_{1} \oplus A_{1} \oplus A_{1} \supset$

$\supset\left(A_{1}\right)_{14} \oplus A_{1} \oplus A_{1} \oplus A_{1} \supset\left(A_{1}\right)_{24} \oplus A_{1} \oplus A_{1}$.

\begin{tabular}{|c|c|c|c|c|c|c|c|c|c|c|c|c|c|}
\hline \multicolumn{2}{|l|}{$B_{6}$} & \multicolumn{2}{|l|}{$D_{6}$} & \multicolumn{2}{|c|}{$\left(A_{1}\right)^{2} \oplus D_{4}$} & \multicolumn{2}{|c|}{$\left(A_{1}\right)^{3} \oplus C_{2}$} & \multicolumn{2}{|l|}{$\left(A_{1}\right)^{5}$} & \multicolumn{2}{|l|}{$\left(A_{1}\right)^{4}$} & \multicolumn{2}{|l|}{$\left(A_{1}\right)^{3}$} \\
\hline Peso & $d$ & Peso & $d$ & Peso & $d$ & Peso & $d$ & Peso & $d$ & Peso & $d$ & Peso & $d$ \\
\hline \multirow[t]{14}{*}{ (000001) } & \multirow[t]{14}{*}{64} & \multirow[t]{7}{*}{$(000010)$} & \multirow[t]{7}{*}{32} & \multirow[t]{4}{*}{$(0,1,0010)$} & \multirow[t]{4}{*}{16} & \multirow[t]{3}{*}{$(0,1,0,01)$} & \multirow[t]{3}{*}{10} & \multirow[t]{2}{*}{$(0,1,0,1,1)$} & \multirow[t]{2}{*}{8} & \multirow[t]{2}{*}{$(1,1,0,1)$} & \multirow[t]{2}{*}{8} & $(2,1,0)$ & 6 \\
\hline & & & & & & & & & & & & $(0,1,0)$ & 2 \\
\hline & & & & & & & & $(0,1,0,0,0)$ & 2 & $(0,1,0,0)$ & 2 & $(1,0,0)$ & 2 \\
\hline & & & & & & $(0,1,2,00)$ & 6 & $(0,1,2,0,0)$ & 6 & $(0,1,2,0)$ & 6 & $(1,0,2)$ & 6 \\
\hline & & & & \multirow[t]{3}{*}{$(1,0,0001)$} & \multirow[t]{3}{*}{16} & \multirow[t]{3}{*}{$(1,0,1,10)$} & \multirow[t]{3}{*}{16} & \multirow[t]{2}{*}{$(1,0,1,1,0)$} & \multirow[t]{2}{*}{8} & $(2,0,1,0)$ & 6 & $(2,0,1)$ & 6 \\
\hline & & & & & & & & & & $(0,0,1,0)$ & 2 & $(0,0,1)$ & 2 \\
\hline & & & & & & & & $(1,0,1,0,1)$ & 8 & $(1,0,1,1)$ & 8 & $(1,1,1)$ & 8 \\
\hline & & \multirow[t]{7}{*}{$(000001)$} & \multirow[t]{7}{*}{32} & \multirow[t]{3}{*}{$(0,1,0001)$} & \multirow[t]{3}{*}{16} & \multirow[t]{3}{*}{$(0,1,1,10)$} & \multirow[t]{3}{*}{16} & $(0,1,1,1,0)$ & 8 & $(1,1,1,0)$ & 8 & $(1,1,1)$ & 8 \\
\hline & & & & & & & & \multirow[t]{2}{*}{$(0,1,1,0,1)$} & \multirow[t]{2}{*}{8} & \multirow[t]{2}{*}{$(0,1,1,1)$} & 8 & $(2,0,1)$ & 6 \\
\hline & & & & & & & & & & & & $(1,0,0)$ & 2 \\
\hline & & & & $(1,0,0010)$ & 16 & $(1,0,0,01)$ & 10 & $(1,0,0,1,1)$ & 8 & $(2,0,0,1)$ & 6 & $(1,2,0)$ & 6 \\
\hline & & & & & & & & & & $(0,0,0,1)$ & 2 & $(1,0,0)$ & 2 \\
\hline & & & & & & & & $(1,0,0,0,0)$ & 2 & $(1,0,0,0)$ & 2 & $(0,1,0)$ & 2 \\
\hline & & & & & & $(1,0,2,00)$ & 6 & $(1,0,2,0,0)$ & 6 & $(1,0,2,0)$ & 6 & $(0,1,2)$ & 6 \\
\hline 1 S.I. & & 2 S.I. & & 4 S.I. & & 6 S.I. & & 10 S.I. & & 12 S.I. & & 14 S.I. & \\
\hline
\end{tabular}


9. $B_{6} \supset D_{6} \supset A_{1} \oplus C_{3} \supset A_{1} \oplus A_{1} \oplus C_{2} \supset A_{1} \oplus A_{1} \oplus A_{1} \supset\left(A_{1}\right)_{23} \oplus A_{1}$.

\begin{tabular}{|c|c|c|c|c|c|c|c|c|c|c|c|}
\hline \multicolumn{2}{|l|}{$B_{6}$} & \multicolumn{2}{|l|}{$D_{6}$} & \multicolumn{2}{|c|}{$A_{1} \oplus C_{3}$} & \multicolumn{2}{|c|}{$\left(A_{1}\right)^{2} \oplus C_{2}$} & \multicolumn{2}{|c|}{$\left(A_{1}\right)^{3}$} & \multicolumn{2}{|c|}{$\left(A_{1}\right)^{2}$} \\
\hline Peso & $d$ & Peso & $d$ & Peso & $d$ & Peso & $d$ & Peso & $d$ & Peso & $d$ \\
\hline \multirow[t]{10}{*}{ (000001) } & \multirow{10}{*}{64} & \multirow{5}{*}{$(000010)$} & \multirow[t]{5}{*}{32} & \multirow{3}{*}{$(0,001)$} & \multirow[t]{3}{*}{14} & \multirow[t]{2}{*}{$(0,1,01)$} & \multirow[t]{2}{*}{10} & \multirow[t]{2}{*}{$(0,1,4)$} & \multirow[t]{2}{*}{10} & $(5,0)$ & 6 \\
\hline & & & & & & & & & & $(3,0)$ & 4 \\
\hline & & & & & & $(0,0,10)$ & 4 & $(0,0,3)$ & 4 & $(3,0)$ & 4 \\
\hline & & & & \multirow[t]{2}{*}{$(2,100)$} & \multirow[t]{2}{*}{18} & $(2,0,10)$ & 12 & $(2,0,3)$ & 12 & $(3,2)$ & 12 \\
\hline & & & & & & $(2,1,00)$ & 6 & $(2,1,0)$ & 6 & $(1,2)$ & 6 \\
\hline & & \multirow[t]{5}{*}{ (000001) } & \multirow[t]{5}{*}{32} & \multirow[t]{4}{*}{$(1,010)$} & \multirow[t]{4}{*}{28} & $(1,0,01)$ & 10 & $(1,0,4)$ & 10 & $(4,1)$ & 10 \\
\hline & & & & & & \multirow[t]{2}{*}{$(1,1,10)$} & \multirow[t]{2}{*}{16} & \multirow[t]{2}{*}{$(1,1,3)$} & \multirow[t]{2}{*}{16} & $(4,1)$ & 10 \\
\hline & & & & & & & & & & $(2,1)$ & 6 \\
\hline & & & & & & $(1,0,00)$ & 2 & $(1,0,0)$ & 2 & $(0,1)$ & 2 \\
\hline & & & & $(3,000)$ & 4 & $(3,0,00)$ & 4 & $(3,0,0)$ & 4 & $(0,3)$ & 4 \\
\hline \multicolumn{2}{|l|}{1 S.I. } & \multicolumn{2}{|l|}{2 S.I. } & \multicolumn{2}{|c|}{4 S.I. } & \multicolumn{2}{|l|}{8 S.I. } & \multicolumn{2}{|c|}{8 S.I. } & \multicolumn{2}{|c|}{10 S.I. } \\
\hline
\end{tabular}

10. $B_{6} \supset C_{2} \oplus D_{4} \supset C_{2} \oplus A_{1} \oplus C_{2} \supset A_{1} \oplus A_{1} \oplus A_{1} \supset\left(A_{1}\right)_{13} \oplus A_{1}$.

\begin{tabular}{|c|c|c|c|c|c|c|c|c|c|}
\hline \multicolumn{2}{|l|}{$B_{6}$} & \multicolumn{2}{|c|}{$C_{2} \oplus D_{4}$} & \multicolumn{2}{|c|}{$C_{2} \oplus A_{1} \oplus C_{2}$} & \multicolumn{2}{|c|}{$\left(A_{1}\right)^{3}$} & \multicolumn{2}{|c|}{$\left(A_{1}\right)^{2}$} \\
\hline Peso & $d$ & Peso & $d$ & Peso & $d$ & Peso & $d$ & Peso & $d$ \\
\hline \multirow[t]{9}{*}{$(000001)$} & \multirow[t]{9}{*}{64} & \multirow[t]{5}{*}{$(10,0010)$} & \multirow[t]{5}{*}{32} & \multirow[t]{4}{*}{$(10,0,01)$} & \multirow[t]{4}{*}{20} & \multirow[t]{4}{*}{$(3,0,4)$} & \multirow[t]{4}{*}{20} & $(7,0)$ & 8 \\
\hline & & & & & & & & $(5,0)$ & 6 \\
\hline & & & & & & & & $(3,0)$ & 4 \\
\hline & & & & & & & & $(1,0)$ & 2 \\
\hline & & & & $(10,2,00)$ & 12 & $(3,2,0)$ & 12 & $(3,2)$ & 12 \\
\hline & & \multirow[t]{4}{*}{$(10,0001)$} & \multirow[t]{4}{*}{32} & \multirow[t]{4}{*}{$(10,1,10)$} & \multirow[t]{4}{*}{32} & \multirow[t]{4}{*}{$(3,1,3)$} & \multirow[t]{4}{*}{32} & $(6,1)$ & 14 \\
\hline & & & & & & & & $(4,1)$ & 10 \\
\hline & & & & & & & & $(2,1)$ & 6 \\
\hline & & & & & & & & $(0,1)$ & 2 \\
\hline \multicolumn{2}{|l|}{1 S.I. } & \multicolumn{2}{|l|}{2 S.I. } & \multicolumn{2}{|l|}{3 S.I. } & \multicolumn{2}{|c|}{3 S.I. } & \multicolumn{2}{|c|}{9 S.I. } \\
\hline
\end{tabular}


11. $B_{6} \supset D_{6} \supset A_{1} \oplus C_{3} \supset A_{1} \oplus A_{1} \oplus C_{2} \supset A_{1} \oplus A_{1} \oplus A_{1} \oplus A_{1} \supset\left(A_{1}\right)_{23} \oplus A_{1} \oplus A_{1} \supset\left(A_{1}\right)_{23} \oplus A_{1}$.

\begin{tabular}{|c|c|c|c|c|c|c|c|c|c|c|c|c|c|}
\hline \multicolumn{2}{|l|}{$B_{6}$} & \multicolumn{2}{|l|}{$D_{6}$} & \multicolumn{2}{|c|}{$A_{1} \oplus C_{3}$} & \multicolumn{2}{|c|}{$\left(A_{1}\right)^{2} \oplus C_{2}$} & \multicolumn{2}{|l|}{$\left(A_{1}\right)^{4}$} & \multicolumn{2}{|c|}{$\left(A_{1}\right)^{3}$} & \multicolumn{2}{|c|}{$\left(A_{1}\right)^{2}$} \\
\hline Peso & $d$ & Peso & $d$ & Peso & $d$ & Peso & $d$ & Peso & $d$ & Peso & $d$ & Peso & $d$ \\
\hline \multirow[t]{18}{*}{ (000001) } & \multirow[t]{18}{*}{64} & \multirow[t]{9}{*}{ (000010) } & \multirow[t]{9}{*}{32} & \multirow[t]{6}{*}{$(0,001)$} & \multirow[t]{6}{*}{14} & \multirow[t]{4}{*}{$(0,1,01)$} & \multirow[t]{4}{*}{10} & \multirow[t]{3}{*}{$(0,1,1,1)$} & \multirow[t]{3}{*}{8} & \multirow[t]{2}{*}{$(2,1,1)$} & \multirow[t]{2}{*}{6} & $(3,0)$ & 4 \\
\hline & & & & & & & & & & & & $(1,0)$ & 2 \\
\hline & & & & & & & & & & $(0,0,1)$ & 2 & $(1,0)$ & 2 \\
\hline & & & & & & & & $(0,1,0,0)$ & 2 & $(1,0,0)$ & 2 & $(1,0)$ & 2 \\
\hline & & & & & & \multirow[t]{2}{*}{$(0,0,10)$} & \multirow[t]{2}{*}{4} & $(0,0,1,0)$ & 2 & $(1,0,0)$ & 2 & $(1,0)$ & 2 \\
\hline & & & & & & & & $(0,0,0,1)$ & 2 & $(0,0,1)$ & 2 & $(1,0)$ & 2 \\
\hline & & & & \multirow[t]{3}{*}{$(2,100)$} & \multirow[t]{3}{*}{18} & \multirow[t]{2}{*}{$(2,0,10)$} & \multirow[t]{2}{*}{12} & $(2,0,1,0)$ & 6 & $(1,2,0)$ & 6 & $(1,2)$ & 6 \\
\hline & & & & & & & & $(2,0,0,1)$ & 6 & $(0,2,1)$ & 6 & $(1,2)$ & 6 \\
\hline & & & & & & $(2,0,00)$ & 6 & $(2,1,0,0)$ & 6 & $(1,2,0)$ & 6 & $(1,2)$ & 6 \\
\hline & & \multirow[t]{9}{*}{ (000001) } & \multirow[t]{9}{*}{32} & \multirow[t]{8}{*}{$(0,010)$} & \multirow[t]{8}{*}{28} & \multirow[t]{3}{*}{$(1,0,01)$} & \multirow[t]{3}{*}{10} & \multirow[t]{2}{*}{$(1,0,1,1)$} & 8 & $(1,1,1)$ & 8 & $(2,1)$ & 6 \\
\hline & & & & & & & & & & & & $(0,1)$ & 2 \\
\hline & & & & & & & & $(1,0,0,0)$ & 2 & $(0,1,0)$ & 2 & $(0,1)$ & 2 \\
\hline & & & & & & $(1,1,10)$ & 16 & $(1,1,1,0)$ & 8 & $(2,1,0)$ & 6 & $(2,1)$ & 6 \\
\hline & & & & & & & & & & $(0,1,0)$ & 2 & $(0,1)$ & 2 \\
\hline & & & & & & & & $(1,1,0,1)$ & 8 & $(1,1,1)$ & 8 & $(2,1)$ & 6 \\
\hline & & & & & & & & & & & & $(0,1)$ & 2 \\
\hline & & & & & & $(1,0,00)$ & 2 & $(1,0,0,0)$ & 2 & $(0,1,0)$ & 2 & $(0,1)$ & 2 \\
\hline & & & & $(3,000)$ & 4 & $(3,0,00)$ & 4 & $(3,0,0,0)$ & 4 & $(0,3,0)$ & 4 & $(0,3)$ & 4 \\
\hline 1 S.I. & & 2 S.I. & & 4 S.I & & 8 S.I. & & 13 S.I. & & 15 S.I & & 18 & \\
\hline
\end{tabular}


12. $B_{6} \supset C_{2} \oplus D_{4} \supset C_{2} \oplus A_{1} \oplus C_{2} \supset A_{1} \oplus A_{1} \oplus A_{1} \oplus A_{1} \supset\left(A_{1}\right)_{12} \oplus A_{1} \oplus A_{1} \supset\left(A_{1}\right)_{23} \oplus A_{1}$.

\begin{tabular}{|c|c|c|c|c|c|c|c|c|c|c|c|}
\hline \multicolumn{2}{|l|}{$B_{6}$} & \multicolumn{2}{|c|}{$C_{2} \oplus D_{4}$} & \multicolumn{2}{|c|}{$C_{2} \oplus A_{1} \oplus C_{2}$} & \multicolumn{2}{|c|}{$\left(A_{1}\right)^{4}$} & \multicolumn{2}{|c|}{$\left(A_{1}\right)^{3}$} & \multicolumn{2}{|c|}{$\left(A_{1}\right)^{2}$} \\
\hline Peso & $d$ & Peso & $d$ & Peso & $d$ & Peso & $d$ & Peso & $d$ & Peso & $d$ \\
\hline \multirow[t]{10}{*}{ (000001) } & \multirow[t]{10}{*}{64} & \multirow[t]{6}{*}{$(10,0010)$} & \multirow[t]{6}{*}{32} & \multirow[t]{3}{*}{$(10,0,01)$} & \multirow[t]{3}{*}{20} & \multirow[t]{2}{*}{$(3,0,1,1)$} & \multirow[t]{2}{*}{16} & \multirow[t]{2}{*}{$(3,1,1)$} & \multirow[t]{2}{*}{16} & $(2,3)$ & 12 \\
\hline & & & & & & & & & & $(0,3)$ & 4 \\
\hline & & & & & & $(3,0,0,0)$ & 4 & $(3,0,0)$ & 4 & $(0,3)$ & 4 \\
\hline & & & & \multirow[t]{3}{*}{$(10,2,00)$} & \multirow[t]{3}{*}{12} & \multirow[t]{3}{*}{$(3,2,0,0)$} & \multirow[t]{3}{*}{12} & $(5,0,0)$ & 6 & $(0,5)$ & 6 \\
\hline & & & & & & & & $(3,0,0)$ & 4 & $(0,3)$ & 4 \\
\hline & & & & & & & & $(1,0,0)$ & 2 & $(0,1)$ & 2 \\
\hline & & \multirow[t]{4}{*}{$(10,0001)$} & \multirow[t]{4}{*}{32} & \multirow[t]{4}{*}{$(10,1,10)$} & \multirow[t]{4}{*}{32} & \multirow[t]{2}{*}{$(3,1,1,0)$} & \multirow[t]{2}{*}{16} & $(4,1,0)$ & 10 & $(1,4)$ & 10 \\
\hline & & & & & & & & $(2,1,0)$ & 6 & $(1,2)$ & 6 \\
\hline & & & & & & \multirow[t]{2}{*}{$(3,1,0,1)$} & \multirow[t]{2}{*}{16} & $(4,0,1)$ & 10 & $(1,4)$ & 10 \\
\hline & & & & & & & & $(2,0,1)$ & 6 & $(1,2)$ & 6 \\
\hline \multicolumn{2}{|c|}{1 S.I. } & \multicolumn{2}{|l|}{2 S.I. } & \multicolumn{2}{|l|}{3 S.I. } & \multicolumn{2}{|l|}{5 S.I. } & \multicolumn{2}{|c|}{6 S.I. } & \multicolumn{2}{|c|}{7 S.I. } \\
\hline
\end{tabular}

13. $B_{6} \supset A_{1} \oplus A_{1} \oplus B_{4} \supset A_{1} \oplus A_{1} \oplus A_{1} \oplus A_{1} \supset\left(A_{1}\right)_{13} \oplus A_{1} \oplus A_{1} \supset\left(A_{1}\right)_{12} \oplus A_{1}$.

\begin{tabular}{|c|c|c|c|c|c|c|c|c|c|}
\hline \multicolumn{2}{|l|}{$B_{6}$} & \multicolumn{2}{|c|}{$\left(A_{1}\right)^{2} \oplus B_{4}$} & \multicolumn{2}{|c|}{$\left(A_{1}\right)^{4}$} & \multicolumn{2}{|c|}{$\left(A_{1}\right)^{3}$} & \multicolumn{2}{|c|}{$\left(A_{1}\right)^{2}$} \\
\hline Peso & $d$ & Peso & $d$ & Peso & $d$ & Peso & $d$ & Peso & $d$ \\
\hline \multirow[t]{8}{*}{ (000001) } & \multirow[t]{8}{*}{64} & \multirow[t]{4}{*}{$(1,0,0001)$} & \multirow[t]{4}{*}{32} & \multirow[t]{2}{*}{$(1,0,3,1)$} & \multirow[t]{2}{*}{16} & $(4,0,1)$ & 10 & $(1,4)$ & 10 \\
\hline & & & & & & $(2,0,1)$ & 6 & $(1,2)$ & 6 \\
\hline & & & & \multirow[t]{2}{*}{$(1,0,1,3)$} & \multirow[t]{2}{*}{16} & $(2,0,3)$ & 12 & $(3,2)$ & 12 \\
\hline & & & & & & $(0,0,3)$ & 4 & $(3,0)$ & 4 \\
\hline & & \multirow[t]{4}{*}{$(0,1,0001)$} & \multirow[t]{4}{*}{32} & \multirow[t]{2}{*}{$(0,1,3,1)$} & \multirow[t]{2}{*}{16} & \multirow[t]{2}{*}{$(3,1,1)$} & \multirow[t]{2}{*}{16} & $(2,3)$ & 12 \\
\hline & & & & & & & & $(3,0)$ & 4 \\
\hline & & & & \multirow[t]{2}{*}{$(0,1,1,3)$} & \multirow[t]{2}{*}{16} & \multirow[t]{2}{*}{$(1,1,3)$} & \multirow[t]{2}{*}{16} & $(4,1)$ & 10 \\
\hline & & & & & & & & $(2,1)$ & 6 \\
\hline \multicolumn{2}{|l|}{1 S.I. } & \multicolumn{2}{|l|}{2 S.I. } & \multicolumn{2}{|l|}{4 S.I. } & \multicolumn{2}{|c|}{6 S.I. } & \multicolumn{2}{|c|}{8 S.I. } \\
\hline
\end{tabular}





\section{Cadeias Sobreviventes de $D_{7}$}

Este apêndice contém as cadeias sobreviventes de $D_{7}=\mathfrak{s o}(14)$. A primeira tabela lista as cadeias e indica a págine em que se encontram. 


\begin{tabular}{|c|c|c|}
\hline Número & Página & Cadeia \\
\hline 1 & 98 & $\begin{aligned} D_{7} & \supset A_{3} \oplus D_{4} \supset A_{3} \oplus A_{1} \oplus A_{1} \oplus A_{1} \oplus A_{1} \supset \\
& \supset A_{2} \oplus A_{1} \oplus A_{1} \oplus A_{1} \oplus A_{1} \supset \tilde{A}_{1} \oplus A_{1} \oplus A_{1} \oplus A_{1} \oplus A_{1}\end{aligned}$ \\
\hline 2 & 98 & $\begin{aligned} D_{7} & \supset A_{1} \oplus B_{5} \supset A_{1} \oplus A_{1} \oplus A_{1} \oplus B_{3} \supset \\
& \supset A_{1} \oplus A_{1} \oplus A_{1} \oplus G_{2} \supset A_{1} \oplus A_{1} \oplus A_{1} \oplus A_{1}\end{aligned}$ \\
\hline 3 & 99 & $\begin{aligned} D_{7} & \supset A_{3} \oplus D_{4} \supset A_{3} \oplus A_{1} \oplus C_{2} \supset A_{2} \oplus A_{1} \oplus C_{2} \supset \\
& \supset \tilde{A}_{1} \oplus A_{1} \oplus C_{2} \supset A_{1} \oplus A_{1} \oplus A_{1} \oplus A_{1}\end{aligned}$ \\
\hline 4 & 99 & $\begin{aligned} D_{7} & \supset B_{3} \oplus B_{3} \supset B_{3} \oplus G_{2} \supset G_{2} \oplus G_{2} \supset \\
& \supset G_{2} \oplus A_{1} \oplus A_{1} \supset A_{1} \oplus A_{1} \oplus A_{1} \oplus A_{1}\end{aligned}$ \\
\hline 5 & 100 & $\begin{aligned} D_{7} & \supset A_{1} \oplus A_{1} \oplus D_{5} \supset A_{1} \oplus A_{1} \oplus D_{4} \supset A_{1} \oplus A_{1} \oplus A_{3} \supset \\
& \supset A_{1} \oplus A_{1} \oplus A_{1} \oplus A_{1}\end{aligned}$ \\
\hline 6 & 100 & $\begin{aligned} D_{7} & \supset A_{1} \oplus A_{1} \oplus D_{5} \supset A_{1} \oplus A_{1} \oplus A_{4} \supset \\
& \supset A_{1} \oplus A_{1} \oplus A_{1} \oplus A_{2} \supset A_{1} \oplus A_{1} \oplus A_{1} \oplus \tilde{A}_{1}\end{aligned}$ \\
\hline 7 & 101 & $\begin{aligned} D_{7} & \supset A_{1} \oplus A_{1} \oplus D_{5} \supset A_{1} \oplus A_{1} \oplus C_{2} \supset \\
& \supset A_{1} \oplus A_{1} \oplus A_{1} \oplus A_{1}\end{aligned}$ \\
\hline 8 & 101 & $\begin{aligned} D_{7} & \supset A_{3} \oplus D_{4} \supset A_{3} \oplus A_{1} \oplus C_{2} \supset A_{2} \oplus A_{1} \oplus C_{2} \supset \\
& \supset \tilde{A}_{1} \oplus A_{1} \oplus C_{2} \supset A_{1} \oplus A_{1} \oplus A_{1}\end{aligned}$ \\
\hline 9 & 102 & $\begin{aligned} D_{7} & \supset B_{3} \oplus B_{3} \supset B_{3} \oplus G_{2} \supset G_{2} \oplus G_{2} \supset \\
& \supset G_{2} \oplus A_{1} \supset A_{1} \oplus A_{1} \oplus A_{1}\end{aligned}$ \\
\hline
\end{tabular}




\begin{tabular}{|c|c|c|c|}
\hline Número & Página & & Cadeia \\
\hline 10 & 102 & $D_{7}$ & $\begin{array}{l}\supset A_{3} \oplus D_{4} \supset A_{3} \oplus A_{1} \oplus C_{2} \supset C_{2} \oplus A_{1} \oplus C_{2} \supset \\
\supset\left(C_{2}\right)_{\operatorname{diag}} \oplus A_{1} \supset A_{1} \oplus A_{1} \oplus A_{1}\end{array}$ \\
\hline 11 & 103 & & $\begin{array}{l}\supset A_{3} \oplus D_{4} \supset A_{3} \oplus A_{1} \oplus C_{2} \supset C_{2} \oplus A_{1} \oplus C_{2} \supset \\
\supset A_{1} \oplus A_{1} \oplus A_{1} \oplus C_{2} \supset A_{1} \oplus A_{1} \oplus A_{1} \oplus A_{1} \oplus A_{1} \supset \\
\supset\left(A_{1}\right)_{14} \oplus A_{1} \oplus A_{1} \oplus A_{1}\end{array}$ \\
\hline 12 & 103 & $D_{7}$ & $\begin{array}{l}\supset A_{3} \oplus D_{4} \supset A_{3} \oplus A_{1} \oplus C_{2} \supset A_{1} \oplus A_{1} \oplus A_{1} \oplus C_{2} \supset \\
\supset A_{1} \oplus A_{1} \oplus A_{1} \oplus A_{1} \oplus A_{1} \supset\left(A_{1}\right)_{13} \oplus A_{1} \oplus A_{1} \oplus A_{1}\end{array}$ \\
\hline 13 & 104 & & $\begin{array}{l}\supset A_{3} \oplus D_{4} \supset A_{3} \oplus A_{1} \oplus C_{2} \supset A_{1} \oplus A_{1} \oplus A_{1} \oplus C_{2} \supset \\
\supset A_{1} \oplus A_{1} \oplus A_{1} \oplus A_{1} \oplus A_{1} \supset\left(A_{1}\right)_{14} \oplus A_{1} \oplus A_{1} \oplus A_{1}\end{array}$ \\
\hline 14 & 104 & $D_{7}$ & $\begin{array}{l}\supset A_{3} \oplus D_{4} \supset A_{3} \oplus A_{1} \oplus C_{2} \supset A_{1} \oplus A_{1} \oplus A_{1} \oplus C_{2} \supset \\
\supset A_{1} \oplus A_{1} \oplus A_{1} \oplus A_{1} \oplus A_{1} \supset\left(A_{1}\right)_{45} \oplus A_{1} \oplus A_{1} \oplus A_{1}\end{array}$ \\
\hline 15 & 105 & & $\begin{array}{l}\supset A_{3} \oplus D_{4} \supset A_{3} \oplus A_{1} \oplus A_{1} \oplus A_{1} \oplus A_{1} \supset \\
\supset A_{2} \oplus A_{1} \oplus A_{1} \oplus A_{1} \oplus A_{1} \supset \tilde{A}_{1} \oplus A_{1} \oplus A_{1} \oplus A_{1} \oplus A_{1} \supset \\
\supset\left(A_{1}\right)_{12} \oplus A_{1} \oplus A_{1} \oplus A_{1}\end{array}$ \\
\hline 16 & 106 & $D_{7}$ & $\begin{array}{l}\supset A_{3} \oplus D_{4} \supset A_{3} \oplus A_{1} \oplus C_{2} \supset A_{2} \oplus A_{1} \oplus C_{2} \supset \\
\supset A_{1} \oplus A_{1} \oplus C_{2} \supset A_{1} \oplus A_{1} \oplus A_{1} \oplus A_{1} \supset\left(A_{1}\right)_{13} \oplus A_{1} \oplus A_{1}\end{array}$ \\
\hline 17 & 107 & $D_{7}$ & $\begin{array}{l}\supset A_{3} \oplus D_{4} \supset A_{3} \oplus A_{1} \oplus C_{2} \supset A_{2} \oplus A_{1} \oplus C_{2} \supset \\
\supset \tilde{A}_{1} \oplus A_{1} \oplus C_{2} \supset A_{1} \oplus A_{1} \oplus A_{1} \oplus A_{1} \supset\left(A_{1}\right)_{13} \oplus A_{1} \oplus A_{1}\end{array}$ \\
\hline 18 & 107 & $D_{7}$ & $\begin{array}{l}\supset A_{3} \oplus D_{4} \supset A_{3} \oplus A_{1} \oplus C_{2} \supset C_{2} \oplus A_{1} \oplus C_{2} \supset \\
\supset A_{1} \oplus A_{1} \oplus A_{1} \oplus C_{2} \supset A_{1} \oplus A_{1} \oplus A_{1} \oplus A_{1} \supset\left(A_{1}\right)_{14} \oplus A_{1} \oplus A_{1}\end{array}$ \\
\hline 19 & 108 & $D_{7}$ & $\begin{array}{l}\supset A_{3} \oplus D_{4} \supset A_{3} \oplus A_{1} \oplus C_{2} \supset C_{2} \oplus A_{1} \oplus C_{2} \supset \\
\supset A_{1} \oplus A_{1} \oplus C_{2} \supset A_{1} \oplus A_{1} \oplus A_{1} \oplus A_{1} \supset\left(A_{1}\right)_{13} \oplus A_{1} \oplus A_{1}\end{array}$ \\
\hline
\end{tabular}




\begin{tabular}{|c|c|c|c|}
\hline Número & Página & & Cadeia \\
\hline 20 & 108 & $D_{7}$ & $\begin{array}{l}\supset A_{3} \oplus D_{4} \supset A_{3} \oplus A_{1} \oplus C_{2} \supset C_{2} \oplus A_{1} \oplus C_{2} \supset \\
\supset A_{1} \oplus A_{1} \oplus C_{2} \supset A_{1} \oplus A_{1} \oplus A_{1} \oplus A_{1} \supset\left(A_{1}\right)_{34} \oplus A_{1} \oplus A_{1}\end{array}$ \\
\hline 21 & 109 & & $\begin{array}{l}\supset A_{3} \oplus D_{4} \supset A_{3} \oplus A_{1} \oplus C_{2} \supset A_{1} \oplus A_{1} \oplus A_{1} \oplus C_{2} \supset \\
\supset A_{1} \oplus A_{1} \oplus A_{1} \oplus A_{1} \supset\left(A_{1}\right)_{13} \oplus A_{1} \oplus A_{1}\end{array}$ \\
\hline 22 & 109 & & $\begin{array}{l}\supset B_{3} \oplus B_{3} \supset B_{3} \oplus G_{2} \supset G_{2} \oplus G_{2} \supset \\
\supset G_{2} \oplus A_{1} \oplus A_{1} \supset A_{1} \oplus A_{1} \oplus A_{1} \oplus A_{1} \supset\left(A_{1}\right)_{13} \oplus A_{1} \oplus A_{1}\end{array}$ \\
\hline 23 & 110 & $D_{7}$ & $\begin{array}{l}\supset B_{3} \oplus B_{3} \supset B_{3} \oplus G_{2} \supset G_{2} \oplus G_{2} \supset \\
\supset G_{2} \oplus A_{1} \oplus A_{1} \supset A_{1} \oplus A_{1} \oplus A_{1} \oplus A_{1} \supset\left(A_{1}\right)_{14} \oplus A_{1} \oplus A_{1}\end{array}$ \\
\hline 24 & 111 & & $\begin{array}{l}\supset A_{1} \oplus A_{1} \oplus D_{5} \supset A_{1} \oplus A_{1} \oplus A_{4} \supset A_{1} \oplus A_{1} \oplus A_{3} \supset \\
\supset A_{1} \oplus A_{1} \oplus A_{1} \oplus A_{1} \supset\left(A_{1}\right)_{13} \oplus A_{1} \oplus A_{1}\end{array}$ \\
\hline 25 & 112 & $D_{7}$ & $\begin{array}{l}\supset A_{1} \oplus A_{1} \oplus D_{5} \supset A_{1} \oplus A_{1} \oplus A_{4} \supset A_{1} \oplus A_{1} \oplus A_{1} \oplus A_{2} \supset \\
\supset A_{1} \oplus A_{1} \oplus A_{1} \oplus \tilde{A}_{1} \supset\left(A_{1}\right)_{13} \oplus A_{1} \oplus A_{1}\end{array}$ \\
\hline 26 & 113 & $D_{7}$ & $\begin{array}{l}\supset A_{1} \oplus A_{1} \oplus D_{5} \supset A_{1} \oplus A_{1} \oplus A_{4} \supset A_{1} \oplus A_{1} \oplus A_{1} \oplus A_{2} \supset \\
\supset A_{1} \oplus A_{1} \oplus A_{1} \oplus \tilde{A}_{1} \supset\left(A_{1}\right)_{14} \oplus A_{1} \oplus A_{1}\end{array}$ \\
\hline 27 & 114 & $D_{7}$ & $\begin{array}{l}\supset A_{1} \oplus A_{1} \oplus D_{5} \supset A_{1} \oplus A_{1} \oplus A_{4} \supset A_{1} \oplus A_{1} \oplus A_{1} \oplus A_{2} \supset \\
\supset A_{1} \oplus A_{1} \oplus A_{1} \oplus \tilde{A}_{1} \supset\left(A_{1}\right)_{34} \oplus A_{1} \oplus A_{1}\end{array}$ \\
\hline 28 & 115 & $D_{7}$ & $\begin{array}{l}\supset A_{3} \oplus D_{4} \supset A_{3} \oplus A_{1} \oplus C_{2} \supset C_{2} \oplus A_{1} \oplus C_{2} \supset A_{1} \oplus A_{1} \oplus A_{1} \oplus C_{2} \supset \\
\supset A_{1} \oplus A_{1} \oplus A_{1} \oplus A_{1} \oplus A_{1} \supset\left(A_{1}\right)_{14} \oplus A_{1} \oplus A_{1} \oplus A_{1} \supset \\
\supset\left(A_{1}\right)_{24} \oplus A_{1} \oplus A_{1}\end{array}$ \\
\hline 29 & 116 & & $\begin{array}{l}\supset A_{3} \oplus D_{4} \supset A_{3} \oplus A_{1} \oplus C_{2} \supset A_{1} \oplus A_{1} \oplus A_{1} \oplus C_{2} \supset \\
\supset A_{1} \oplus A_{1} \oplus A_{1} \oplus A_{1} \oplus A_{1} \supset\left(A_{1}\right)_{13} \oplus A_{1} \oplus A_{1} \oplus A_{1} \supset \\
\supset\left(A_{1}\right)_{24} \oplus A_{1} \oplus A_{1}\end{array}$ \\
\hline
\end{tabular}




\begin{tabular}{|c|c|c|}
\hline Número & Página & Cadeia \\
\hline 30 & 116 & $\begin{aligned} D_{7} & \supset A_{3} \oplus D_{4} \supset A_{3} \oplus A_{1} \oplus C_{2} \supset A_{2} \oplus A_{1} \oplus C_{2} \supset \\
& \supset A_{1} \oplus A_{1} \oplus C_{2} \supset A_{1} \oplus A_{1} \oplus A_{1} \supset\left(A_{1}\right)_{12} \oplus A_{1}\end{aligned}$ \\
\hline 31 & 117 & $\begin{aligned} D_{7} & \supset A_{3} \oplus D_{4} \supset A_{3} \oplus A_{1} \oplus C_{2} \supset C_{2} \oplus A_{1} \oplus C_{2} \supset \\
& \supset A_{1} \oplus A_{1} \oplus C_{2} \supset A_{1} \oplus A_{1} \oplus A_{1} \supset\left(A_{1}\right)_{13} \oplus A_{1}\end{aligned}$ \\
\hline 32 & 117 & $\begin{aligned} D_{7} & \supset B_{3} \oplus B_{3} \supset B_{3} \oplus G_{2} \supset G_{2} \oplus G_{2} \supset \\
& \supset G_{2} \oplus A_{1} \supset A_{1} \oplus A_{1} \oplus A_{1} \supset\left(A_{1}\right)_{13} \oplus A_{1}\end{aligned}$ \\
\hline 33 & 118 & $\begin{aligned} D_{7} & \supset A_{3} \oplus D_{4} \supset A_{3} \oplus A_{1} \oplus C_{2} \supset C_{2} \oplus A_{1} \oplus C_{2} \supset A_{1} \oplus A_{1} \oplus A_{1} \oplus C_{2} \supset \\
& \supset A_{1} \oplus A_{1} \oplus A_{1} \oplus A_{1} \supset\left(A_{1}\right)_{13} \oplus A_{1} \oplus A_{1} \supset\left(A_{1}\right)_{23} \oplus A_{1}\end{aligned}$ \\
\hline 34 & 118 & $\begin{aligned} D_{7} & \supset A_{3} \oplus D_{4} \supset A_{3} \oplus A_{1} \oplus C_{2} \supset C_{2} \oplus A_{1} \oplus C_{2} \supset A_{1} \oplus A_{1} \oplus C_{2} \supset \\
& \supset A_{1} \oplus A_{1} \oplus A_{1} \oplus A_{1} \supset\left(A_{1}\right)_{12} \oplus A_{1} \oplus A_{1} \supset\left(A_{1}\right)_{23} \oplus A_{1}\end{aligned}$ \\
\hline 35 & 119 & $\begin{aligned} D_{7} & \supset A_{3} \oplus D_{4} \supset A_{3} \oplus A_{1} \oplus C_{2} \supset A_{1} \oplus A_{1} \oplus A_{1} \oplus C_{2} \supset \\
& \supset A_{1} \oplus A_{1} \oplus A_{1} \oplus A_{1} \supset\left(A_{1}\right)_{13} \oplus A_{1} \oplus A_{1} \supset\left(A_{1}\right)_{23} \oplus A_{1}\end{aligned}$ \\
\hline 36 & 119 & $\begin{aligned} D_{7} & \supset C_{2} \oplus B_{4} \supset C_{2} \oplus A_{1} \oplus A_{1} \supset A_{1} \oplus A_{1} \oplus A_{1} \oplus A_{1} \supset \\
& \supset\left(A_{1}\right)_{13} \oplus A_{1} \oplus A_{1} \supset\left(A_{1}\right)_{23} \oplus A_{1}\end{aligned}$ \\
\hline 37 & 120 & $\begin{aligned} D_{7} & \supset A_{1} \oplus A_{1} \oplus D_{5} \supset A_{1} \oplus A_{1} \oplus A_{4} \supset A_{1} \oplus A_{1} \oplus A_{3} \supset \\
& \supset A_{1} \oplus A_{1} \oplus A_{1} \oplus A_{1} \supset\left(A_{1}\right)_{13} \oplus A_{1} \oplus A_{1} \supset\left(A_{1}\right)_{23} \oplus A_{1}\end{aligned}$ \\
\hline 38 & 120 & $\begin{aligned} D_{7} & \supset C_{2} \oplus B_{4} \supset C_{2} \oplus A_{1} \oplus A_{1} \supset A_{1} \oplus A_{1} \oplus A_{1} \oplus A_{1} \supset \\
& \supset\left(A_{1}\right)_{12} \oplus A_{1} \oplus A_{1} \supset\left(A_{1}\right)_{23} \oplus A_{1}\end{aligned}$ \\
\hline 39 & 121 & $\begin{aligned} D_{7} & \supset A_{1} \oplus A_{1} \oplus D_{5} \supset A_{1} \oplus A_{1} \oplus A_{4} \supset A_{1} \oplus A_{1} \oplus A_{3} \supset \\
& \supset A_{1} \oplus A_{1} \oplus A_{1} \oplus \tilde{A}_{1} \supset\left(A_{1}\right)_{13} \oplus A_{1} \oplus A_{1} \supset\left(A_{1}\right)_{23} \oplus A_{1}\end{aligned}$ \\
\hline
\end{tabular}


1. $D_{7} \supset A_{3} \oplus D_{4} \supset A_{3} \oplus A_{1} \oplus A_{1} \oplus A_{1} \oplus A_{1} \supset A_{2} \oplus A_{1} \oplus A_{1} \oplus A_{1} \oplus A_{1} \supset \tilde{A}_{1} \oplus A_{1} \oplus A_{1} \oplus A_{1} \oplus A_{1}$.

\begin{tabular}{|c|c|c|c|c|c|c|c|c|c|}
\hline \multicolumn{2}{|l|}{$D_{7}$} & \multicolumn{2}{|c|}{$A_{3} \oplus D_{4}$} & \multicolumn{2}{|c|}{$A_{3} \oplus\left(A_{1}\right)^{4}$} & \multicolumn{2}{|c|}{$A_{2} \oplus\left(A_{1}\right)^{4}$} & \multicolumn{2}{|l|}{$\left(A_{1}\right)^{5}$} \\
\hline Peso & $d$ & Peso & $d$ & Peso & $d$ & Peso & $d$ & Peso & $d$ \\
\hline \multirow[t]{8}{*}{$(0000010)$} & \multirow[t]{8}{*}{64} & \multirow[t]{4}{*}{$(001,0010)$} & \multirow[t]{4}{*}{32} & \multirow[t]{2}{*}{$(001,0,1,1,0)$} & \multirow[t]{2}{*}{16} & $(01,0,1,1,0)$ & 12 & $(2,0,1,1,0)$ & 12 \\
\hline & & & & & & $(00,0,1,1,0)$ & 4 & $(0,0,1,1,0)$ & 4 \\
\hline & & & & \multirow[t]{2}{*}{$(001,1,0,0,1)$} & \multirow[t]{2}{*}{16} & $(01,1,0,0,1)$ & 12 & $(2,1,0,0,1)$ & 12 \\
\hline & & & & & & $(00,1,0,0,1)$ & 4 & $(0,1,0,0,1)$ & 4 \\
\hline & & \multirow[t]{4}{*}{$(100,0001)$} & \multirow[t]{4}{*}{32} & \multirow[t]{2}{*}{$(100,0,1,1,0)$} & \multirow[t]{2}{*}{16} & $(10,0,1,0,1)$ & 12 & $(2,0,1,0,1)$ & 12 \\
\hline & & & & & & $(00,0,1,0,1)$ & 4 & $(0,0,1,0,1)$ & 4 \\
\hline & & & & \multirow[t]{2}{*}{$(100,1,0,1,0)$} & \multirow[t]{2}{*}{16} & $(10,1,0,1,0)$ & 12 & $(2,1,0,1,0)$ & 12 \\
\hline & & & & & & $(00,1,0,1,0)$ & 4 & $(0,1,0,1,0)$ & 4 \\
\hline \multicolumn{2}{|l|}{1 S.I. } & \multicolumn{2}{|l|}{2 S.I. } & \multicolumn{2}{|l|}{4 S.I. } & \multicolumn{2}{|l|}{8 S.I. } & \multicolumn{2}{|l|}{8 S.I. } \\
\hline
\end{tabular}

2. $D_{7} \supset A_{1} \oplus B_{5} \supset A_{1} \oplus A_{1} \oplus A_{1} \oplus B_{3} \supset A_{1} \oplus A_{1} \oplus A_{1} \oplus G_{2} \supset A_{1} \oplus A_{1} \oplus A_{1} \oplus A_{1} \oplus A_{1}$.

\begin{tabular}{|c|c|c|c|c|c|c|c|c|c|}
\hline \multicolumn{2}{|l|}{$D_{7}$} & \multicolumn{2}{|c|}{$A_{1} \oplus B_{5}$} & \multicolumn{2}{|c|}{$\left(A_{1}\right)^{3} \oplus B_{3}$} & \multicolumn{2}{|c|}{$\left(A_{1}\right)^{3} \oplus G_{2}$} & \multicolumn{2}{|l|}{$\left(A_{1}\right)^{5}$} \\
\hline Peso & $d$ & Peso & $d$ & Peso & $d$ & Peso & $d$ & Peso & $d$ \\
\hline \multirow[t]{6}{*}{ (0000010) } & \multirow[t]{6}{*}{64} & \multirow[t]{6}{*}{$(1,00001)$} & \multirow[t]{6}{*}{64} & \multirow[t]{3}{*}{$(1,1,0,001)$} & \multirow[t]{3}{*}{32} & \multirow[t]{2}{*}{$(1,1,0,01)$} & \multirow[t]{2}{*}{28} & $(1,1,0,1,1)$ & 16 \\
\hline & & & & & & & & $(1,1,0,0,2)$ & 12 \\
\hline & & & & & & $(1,1,0,00)$ & 4 & $(1,1,0,0,0)$ & 4 \\
\hline & & & & \multirow{3}{*}{$(1,0,1,001)$} & \multirow[t]{3}{*}{32} & \multirow{2}{*}{$(1,0,1,01)$} & \multirow[t]{2}{*}{28} & $(1,0,1,1,1)$ & 16 \\
\hline & & & & & & & & $(1,0,1,0,2)$ & 12 \\
\hline & & & & & & $(1,0,1,00)$ & 4 & $(1,0,1,0,0)$ & 4 \\
\hline \multicolumn{2}{|l|}{1 S.I. } & \multicolumn{2}{|l|}{1 S.I. } & \multicolumn{2}{|l|}{2 S.I. } & \multicolumn{2}{|l|}{4 S.I. } & \multicolumn{2}{|l|}{6 S.I. } \\
\hline
\end{tabular}


3. $D_{7} \supset A_{3} \oplus D_{4} \supset A_{3} \oplus A_{1} \oplus C_{2} \supset A_{2} \oplus A_{1} \oplus C_{2} \supset \tilde{A}_{1} \oplus A_{1} \oplus C_{2} \supset A_{1} \oplus A_{1} \oplus A_{1} \oplus A_{1}$.

\begin{tabular}{|c|c|c|c|c|c|c|c|c|c|c|c|}
\hline \multicolumn{2}{|l|}{$D_{7}$} & \multicolumn{2}{|c|}{$A_{3} \oplus D_{4}$} & \multicolumn{2}{|c|}{$A_{3} \oplus A_{1} \oplus C_{2}$} & \multicolumn{2}{|c|}{$A_{2} \oplus A_{1} \oplus C_{2}$} & \multicolumn{2}{|c|}{$\left(A_{1}\right)^{2} \oplus C_{2}$} & \multicolumn{2}{|l|}{$\left(A_{1}\right)^{4}$} \\
\hline Peso & $d$ & Peso & $d$ & Peso & $d$ & Peso & $d$ & Peso & $d$ & Peso & $d$ \\
\hline \multirow[t]{10}{*}{ (0000010) } & \multirow[t]{10}{*}{64} & \multirow[t]{6}{*}{$(001,0010)$} & \multirow[t]{6}{*}{32} & \multirow[t]{4}{*}{$(001,0,01)$} & \multirow[t]{4}{*}{20} & \multirow[t]{2}{*}{$(01,0,01)$} & \multirow[t]{2}{*}{15} & \multirow[t]{2}{*}{$(2,0,01)$} & \multirow[t]{2}{*}{15} & $(2,0,1,1)$ & 12 \\
\hline & & & & & & & & & & $(2,0,0,0)$ & 3 \\
\hline & & & & & & \multirow[t]{2}{*}{$(00,0,01)$} & \multirow[t]{2}{*}{5} & \multirow[t]{2}{*}{$(0,0,01)$} & \multirow[t]{2}{*}{5} & $(0,0,1,1)$ & 4 \\
\hline & & & & & & & & & & $(0,0,0,0)$ & 1 \\
\hline & & & & \multirow[t]{2}{*}{$(001,2,00)$} & \multirow[t]{2}{*}{12} & $(01,2,00)$ & 9 & $(2,2,00)$ & 9 & $(2,2,0,0)$ & 9 \\
\hline & & & & & & $(00,2,00)$ & 3 & $(0,2,00)$ & 3 & $(0,2,0,0)$ & 3 \\
\hline & & \multirow[t]{4}{*}{$(100,0001)$} & \multirow[t]{4}{*}{32} & \multirow[t]{4}{*}{$(100,1,10)$} & \multirow[t]{4}{*}{32} & \multirow[t]{2}{*}{$(10,1,10)$} & \multirow[t]{2}{*}{24} & \multirow[t]{2}{*}{$(2,1,10)$} & \multirow[t]{2}{*}{24} & $(2,1,1,0)$ & 12 \\
\hline & & & & & & & & & & $(2,1,0,1)$ & 12 \\
\hline & & & & & & \multirow[t]{2}{*}{$(00,1,10)$} & \multirow[t]{2}{*}{8} & \multirow[t]{2}{*}{$(0,1,10)$} & \multirow[t]{2}{*}{8} & $(0,1,1,0)$ & 4 \\
\hline & & & & & & & & & & $(0,1,0,1)$ & 4 \\
\hline \multicolumn{2}{|l|}{1 S.I. } & \multicolumn{2}{|l|}{2 S.I. } & \multicolumn{2}{|l|}{3 S.I. } & 6 S.I. & & 6 S.I & & 10 S.I. & \\
\hline
\end{tabular}

4. $D_{7} \supset B_{3} \oplus B_{3} \supset B_{3} \oplus G_{2} \supset G_{2} \oplus G_{2} \supset G_{2} \oplus A_{1} \oplus A_{1} \supset A_{1} \oplus A_{1} \oplus A_{1} \oplus A_{1}$.

\begin{tabular}{|c|c|c|c|c|c|c|c|c|c|c|c|}
\hline \multicolumn{2}{|l|}{$D_{7}$} & \multicolumn{2}{|c|}{$B_{3} \oplus B_{3}$} & \multicolumn{2}{|c|}{$B_{3} \oplus G_{2}$} & \multicolumn{2}{|c|}{$G_{2} \oplus G_{2}$} & \multicolumn{2}{|c|}{$G_{2} \oplus\left(A_{1}\right)^{2}$} & \multicolumn{2}{|l|}{$\left(A_{1}\right)^{4}$} \\
\hline Peso & $d$ & Peso & $d$ & Peso & $d$ & Peso & $d$ & Peso & $d$ & Peso & $d$ \\
\hline \multirow[t]{9}{*}{ (0000010) } & \multirow[t]{9}{*}{64} & \multirow{9}{*}{$(001,001)$} & \multirow[t]{9}{*}{64} & \multirow[t]{6}{*}{$(001,01)$} & \multirow[t]{6}{*}{56} & \multirow[t]{4}{*}{$(01,01)$} & \multirow[t]{4}{*}{49} & \multirow[t]{2}{*}{$(01,1,1)$} & \multirow[t]{2}{*}{28} & $(1,1,1,1)$ & 16 \\
\hline & & & & & & & & & & $(0,2,1,1)$ & 12 \\
\hline & & & & & & & & \multirow[t]{2}{*}{$(01,0,2)$} & \multirow[t]{2}{*}{21} & $(1,1,0,2)$ & 12 \\
\hline & & & & & & & & & & $(0,2,0,2)$ & 9 \\
\hline & & & & & & \multirow[t]{2}{*}{$(00,01)$} & \multirow[t]{2}{*}{7} & $(00,1,1)$ & 4 & $(0,0,1,1)$ & 4 \\
\hline & & & & & & & & $(00,0,2)$ & 3 & $(0,0,0,2)$ & 3 \\
\hline & & & & \multirow[t]{3}{*}{$(001,00)$} & \multirow[t]{3}{*}{8} & \multirow[t]{2}{*}{$(01,00)$} & \multirow[t]{2}{*}{7} & \multirow[t]{2}{*}{$(01,0,0)$} & \multirow[t]{2}{*}{7} & $(1,1,0,0)$ & 4 \\
\hline & & & & & & & & & & $(0,2,0,0)$ & 3 \\
\hline & & & & & & $(00,00)$ & 1 & $(00,0,0)$ & 1 & $(0,0,0,0)$ & 1 \\
\hline \multicolumn{2}{|l|}{1 S.I. } & \multicolumn{2}{|l|}{1 S.I. } & \multicolumn{2}{|c|}{2 S.I. } & \multicolumn{2}{|c|}{4 S.I. } & \multicolumn{2}{|c|}{6 S.I. } & \multicolumn{2}{|l|}{9 S.I. } \\
\hline
\end{tabular}


5. $D_{7} \supset A_{1} \oplus A_{1} \oplus D_{5} \supset A_{1} \oplus A_{1} \oplus A_{4} \supset A_{1} \oplus A_{1} \oplus A_{3} \supset A_{1} \oplus A_{1} \oplus A_{1} \oplus A_{1}$.

\begin{tabular}{|c|c|c|c|c|c|c|c|c|c|}
\hline \multicolumn{2}{|l|}{$D_{7}$} & \multicolumn{2}{|c|}{$\left(A_{1}\right)^{2} \oplus D_{5}$} & \multicolumn{2}{|c|}{$\left(A_{1}\right)^{2} \oplus A_{4}$} & \multicolumn{2}{|c|}{$\left(A_{1}\right)^{2} \oplus A_{3}$} & \multicolumn{2}{|l|}{$\left(A_{1}\right)^{4}$} \\
\hline Peso & $d$ & Peso & $d$ & Peso & $d$ & Peso & $d$ & Peso & $d$ \\
\hline \multirow[t]{12}{*}{ (0000010) } & \multirow[t]{12}{*}{64} & \multirow[t]{6}{*}{$(0,1,00010)$} & \multirow[t]{6}{*}{32} & \multirow[t]{2}{*}{$(0,1,1000)$} & \multirow[t]{2}{*}{10} & $(0,1,100)$ & 8 & $(0,1,1,1)$ & 8 \\
\hline & & & & & & $(0,1,000)$ & 2 & $(0,1,0,0)$ & 2 \\
\hline & & & & \multirow[t]{3}{*}{$(0,1,0010)$} & \multirow[t]{3}{*}{20} & \multirow[t]{2}{*}{$(0,1,010)$} & \multirow[t]{2}{*}{12} & $(0,1,2,0)$ & 6 \\
\hline & & & & & & & & $(0,1,0,2)$ & 6 \\
\hline & & & & & & $(0,1,001)$ & 8 & $(0,1,1,1)$ & 8 \\
\hline & & & & $(0,1,0000)$ & 2 & $(0,1,000)$ & 2 & $(0,1,0,0)$ & 2 \\
\hline & & \multirow[t]{6}{*}{$(1,0,00001)$} & \multirow[t]{6}{*}{32} & \multirow[t]{2}{*}{$(1,0,0001)$} & \multirow[t]{2}{*}{10} & $(1,0,001)$ & 8 & $(1,0,1,1)$ & 8 \\
\hline & & & & & & $(1,0,000)$ & 2 & $(1,0,0,0)$ & 2 \\
\hline & & & & \multirow[t]{3}{*}{$(1,0,0100)$} & \multirow[t]{3}{*}{20} & \multirow[t]{2}{*}{$(1,0,010)$} & \multirow[t]{2}{*}{12} & $(1,0,2,0)$ & 6 \\
\hline & & & & & & & & $(1,0,0,2)$ & 6 \\
\hline & & & & & & $(1,0,100)$ & 8 & $(1,0,1,1)$ & 8 \\
\hline & & & & $(1,0,0000)$ & 2 & $(1,0,000)$ & 2 & $(1,0,0,0)$ & 2 \\
\hline \multicolumn{2}{|l|}{1 S.I. } & \multicolumn{2}{|l|}{2 S.I. } & \multicolumn{2}{|l|}{6 S.I. } & \multicolumn{2}{|l|}{10 S.I. } & \multicolumn{2}{|l|}{12 S.I. } \\
\hline
\end{tabular}

6. $D_{7} \supset A_{1} \oplus A_{1} \oplus D_{5} \supset A_{1} \oplus A_{1} \oplus A_{4} \supset A_{1} \oplus A_{1} \oplus A_{1} \oplus A_{2} \supset A_{1} \oplus A_{1} \oplus A_{1} \oplus \tilde{A}_{1}$.

\begin{tabular}{|c|c|c|c|c|c|c|c|c|c|}
\hline \multicolumn{2}{|l|}{$D_{7}$} & \multicolumn{2}{|c|}{$\left(A_{1}\right)^{2} \oplus D_{5}$} & \multicolumn{2}{|c|}{$\left(A_{1}\right)^{2} \oplus A_{4}$} & \multicolumn{2}{|c|}{$\left(A_{1}\right)^{3} \oplus A_{2}$} & \multicolumn{2}{|c|}{$\left(A_{1}\right)^{4}$} \\
\hline Peso & $d$ & Peso & $d$ & Peso & $d$ & Peso & $d$ & Peso & $d$ \\
\hline \multirow[t]{12}{*}{ (0000010) } & \multirow[t]{12}{*}{64} & \multirow[t]{6}{*}{$(0,1,00010)$} & \multirow[t]{6}{*}{32} & \multirow[t]{2}{*}{$(0,1,1000)$} & \multirow[t]{2}{*}{10} & $(0,1,0,10)$ & 6 & $(0,1,0,2)$ & 6 \\
\hline & & & & & & $(0,1,1,00)$ & 4 & $(0,1,1,0)$ & 4 \\
\hline & & & & \multirow[t]{3}{*}{$(0,1,0010)$} & \multirow[t]{3}{*}{20} & $(0,1,1,01)$ & 12 & $(0,1,1,2)$ & 12 \\
\hline & & & & & & $(0,1,0,10)$ & 6 & $(0,1,0,2)$ & 6 \\
\hline & & & & & & $(0,1,0,00)$ & 2 & $(0,1,0,0)$ & 2 \\
\hline & & & & $(0,1,0000)$ & 2 & $(0,1,0,00)$ & 2 & $(0,1,0,0)$ & 2 \\
\hline & & \multirow[t]{6}{*}{$(1,0,00001)$} & \multirow[t]{6}{*}{32} & \multirow[t]{2}{*}{$(1,0,0001)$} & \multirow[t]{2}{*}{10} & $(1,0,0,01)$ & 6 & $(1,0,0,2)$ & 6 \\
\hline & & & & & & $(1,0,1,00)$ & 4 & $(1,0,1,0)$ & 4 \\
\hline & & & & \multirow[t]{3}{*}{$(1,0,0100)$} & \multirow[t]{3}{*}{20} & $(1,0,1,10)$ & 12 & $(1,0,1,2)$ & 12 \\
\hline & & & & & & $(1,0,0,01)$ & 6 & $(1,0,0,2)$ & 6 \\
\hline & & & & & & $(1,0,0,00)$ & 2 & $(1,0,0,0)$ & 2 \\
\hline & & & & $(1,0,0000)$ & 2 & $(1,0,0,00)$ & 2 & $(1,0,0,0)$ & 2 \\
\hline \multicolumn{2}{|l|}{1 S.I. } & \multicolumn{2}{|l|}{2 S.I. } & \multicolumn{2}{|l|}{6 S.I. } & \multicolumn{2}{|l|}{12 S.I. } & \multicolumn{2}{|l|}{12 S.I. } \\
\hline
\end{tabular}


7. $D_{7} \supset A_{1} \oplus A_{1} \oplus D_{5} \supset A_{1} \oplus A_{1} \oplus C_{2} \supset A_{1} \oplus A_{1} \oplus A_{1} \oplus A_{1}$.

\begin{tabular}{|c|c|c|c|c|c|c|c|}
\hline \multicolumn{2}{|l|}{$D_{7}$} & \multicolumn{2}{|c|}{$\left(A_{1}\right)^{2} \oplus D_{5}$} & \multicolumn{2}{|c|}{$\left(A_{1}\right)^{2} \oplus C_{2}$} & \multicolumn{2}{|c|}{$\left(A_{1}\right)^{4}$} \\
\hline Peso & $d$ & Peso & $d$ & Peso & $d$ & Peso & $d$ \\
\hline \multirow[t]{8}{*}{ (0000010) } & \multirow[t]{8}{*}{64} & \multirow[t]{4}{*}{$(0,1,00010)$} & \multirow[t]{4}{*}{32} & \multirow[t]{4}{*}{$(0,1,11)$} & \multirow[t]{4}{*}{32} & $(0,1,2,1)$ & 12 \\
\hline & & & & & & $(0,1,1,2)$ & 12 \\
\hline & & & & & & $(0,1,1,0)$ & 4 \\
\hline & & & & & & $(0,1,0,1)$ & 4 \\
\hline & & \multirow[t]{4}{*}{$(1,0,00001)$} & \multirow[t]{4}{*}{32} & \multirow[t]{4}{*}{$(1,0,11)$} & \multirow[t]{4}{*}{32} & $(1,0,2,1)$ & 12 \\
\hline & & & & & & $(1,0,1,2)$ & 12 \\
\hline & & & & & & $(1,0,1,0)$ & 4 \\
\hline & & & & & & $(1,0,0,1)$ & 4 \\
\hline \multicolumn{2}{|l|}{1 S.I. } & \multicolumn{2}{|l|}{2 S.I. } & \multicolumn{2}{|c|}{2 S.I. } & \multicolumn{2}{|l|}{8 S.I. } \\
\hline
\end{tabular}

8. $D_{7} \supset A_{3} \oplus D_{4} \supset A_{3} \oplus A_{1} \oplus C_{2} \supset A_{2} \oplus A_{1} \oplus C_{2} \supset \tilde{A}_{1} \oplus A_{1} \oplus C_{2} \supset A_{1} \oplus A_{1} \oplus A_{1}$.

\begin{tabular}{|c|c|c|c|c|c|c|c|c|c|c|c|}
\hline \multicolumn{2}{|l|}{$D_{7}$} & \multicolumn{2}{|c|}{$A_{3} \oplus D_{4}$} & \multicolumn{2}{|c|}{$A_{3} \oplus A_{1} \oplus C_{2}$} & \multicolumn{2}{|c|}{$A_{2} \oplus A_{1} \oplus C_{2}$} & \multicolumn{2}{|c|}{$\left(A_{1}\right)^{2} \oplus C_{2}$} & \multicolumn{2}{|c|}{$\left(A_{1}\right)^{3}$} \\
\hline Peso & $d$ & Peso & $d$ & Peso & $d$ & Peso & $d$ & Peso & $d$ & Peso & $d$ \\
\hline \multirow[t]{6}{*}{ (0000010) } & \multirow[t]{6}{*}{64} & \multirow[t]{4}{*}{$(001,0010)$} & \multirow[t]{4}{*}{32} & \multirow[t]{2}{*}{$(001,0,01)$} & \multirow[t]{2}{*}{20} & $(01,0,01)$ & 15 & $(2,0,01)$ & 15 & $(2,0,4)$ & 15 \\
\hline & & & & & & $(00,0,01)$ & 5 & $(0,0,01)$ & 5 & $(0,0,4)$ & 5 \\
\hline & & & & \multirow[t]{2}{*}{$(001,2,00)$} & \multirow[t]{2}{*}{12} & $(01,2,00)$ & 9 & $(2,2,00)$ & 9 & $(2,2,0)$ & 9 \\
\hline & & & & & & $(00,2,00)$ & 3 & $(0,2,00)$ & 3 & $(0,2,0)$ & 3 \\
\hline & & \multirow[t]{2}{*}{$(100,0001)$} & \multirow[t]{2}{*}{32} & \multirow[t]{2}{*}{$(100,1,10)$} & \multirow[t]{2}{*}{32} & $(10,1,10)$ & 24 & $(2,1,10)$ & 24 & $(2,1,3)$ & 24 \\
\hline & & & & & & $(00,1,10)$ & 8 & $(0,1,10)$ & 8 & $(0,1,3)$ & 8 \\
\hline \multicolumn{2}{|l|}{1 S.I. } & \multicolumn{2}{|l|}{2 S.I. } & \multicolumn{2}{|l|}{3 S.I. } & \multicolumn{2}{|l|}{6 S.I. } & \multicolumn{2}{|c|}{6 S.I. } & \multicolumn{2}{|c|}{6 S.I. } \\
\hline
\end{tabular}


9. $D_{7} \supset B_{3} \oplus B_{3} \supset B_{3} \oplus G_{2} \supset G_{2} \oplus G_{2} \supset G_{2} \oplus A_{1} \supset A_{1} \oplus A_{1} \oplus A_{1}$.

\begin{tabular}{|c|c|c|c|c|c|c|c|c|c|c|c|}
\hline \multicolumn{2}{|l|}{$D_{7}$} & \multicolumn{2}{|c|}{$B_{3} \oplus B_{3}$} & \multicolumn{2}{|c|}{$B_{3} \oplus G_{2}$} & \multicolumn{2}{|c|}{$G_{2} \oplus G_{2}$} & \multicolumn{2}{|c|}{$G_{2} \oplus A_{1}$} & \multicolumn{2}{|c|}{$\left(A_{1}\right)^{3}$} \\
\hline Peso & $d$ & Peso & $d$ & Peso & $d$ & Peso & $d$ & Peso & $d$ & Peso & $d$ \\
\hline \multirow[t]{6}{*}{ (0000010) } & \multirow[t]{6}{*}{64} & \multirow[t]{6}{*}{$(001,001)$} & \multirow[t]{6}{*}{64} & \multirow[t]{3}{*}{$(001,01)$} & \multirow[t]{3}{*}{49} & \multirow[t]{2}{*}{$(01,01)$} & \multirow[t]{2}{*}{6} & \multirow[t]{2}{*}{$(01,6)$} & \multirow[t]{2}{*}{49} & $(1,1,6)$ & 28 \\
\hline & & & & & & & & & & $(0,2,6)$ & 21 \\
\hline & & & & & & $(00,01)$ & 7 & $(00,6)$ & 7 & $(0,0,6)$ & 7 \\
\hline & & & & \multirow[t]{3}{*}{$(001,00)$} & \multirow[t]{3}{*}{8} & \multirow[t]{2}{*}{$(01,00)$} & \multirow[t]{2}{*}{7} & \multirow[t]{2}{*}{$(01,0)$} & \multirow[t]{2}{*}{7} & $(1,1,0)$ & 4 \\
\hline & & & & & & & & & & $(0,2,0)$ & 3 \\
\hline & & & & & & $(00,00)$ & 1 & $(00,0)$ & 1 & $(0,0,0)$ & 1 \\
\hline \multicolumn{2}{|l|}{1 S.I. } & \multicolumn{2}{|l|}{1 S.I. } & \multicolumn{2}{|c|}{2 S.I. } & \multicolumn{2}{|l|}{4 S.I. } & \multicolumn{2}{|c|}{4 S.I. } & \multicolumn{2}{|c|}{6 S.I. } \\
\hline
\end{tabular}

10. $D_{7} \supset A_{3} \oplus D_{4} \supset A_{3} \oplus A_{1} \oplus C_{2} \supset C_{2} \oplus A_{1} \oplus C_{2} \supset\left(C_{2}\right)_{\operatorname{diag}} \oplus A_{1} \supset A_{1} \oplus A_{1} \oplus A_{1}$.

\begin{tabular}{|c|c|c|c|c|c|c|c|c|c|c|c|}
\hline \multicolumn{2}{|l|}{$D_{7}$} & \multicolumn{2}{|c|}{$A_{3} \oplus D_{4}$} & \multicolumn{2}{|c|}{$A_{3} \oplus A_{1} \oplus C_{2}$} & \multicolumn{2}{|c|}{$C_{2} \oplus A_{1} \oplus C_{2}$} & \multicolumn{2}{|c|}{$\left(C_{2}\right) \oplus A_{1}$} & \multicolumn{2}{|c|}{$\left(A_{1}\right)^{3}$} \\
\hline Peso & $d$ & Peso & $d$ & Peso & $d$ & Peso & $d$ & Peso & $d$ & Peso & $d$ \\
\hline \multirow[t]{14}{*}{$(0000010)$} & \multirow[t]{14}{*}{64} & \multirow[t]{8}{*}{$(001,0010)$} & \multirow[t]{8}{*}{32} & \multirow[t]{6}{*}{$(001,0,01)$} & \multirow[t]{6}{*}{20} & \multirow[t]{6}{*}{$(10,0,01)$} & \multirow[t]{6}{*}{20} & \multirow[t]{4}{*}{$(11,0)$} & \multirow[t]{4}{*}{16} & $(2,1,0)$ & 6 \\
\hline & & & & & & & & & & $(1,2,0)$ & 6 \\
\hline & & & & & & & & & & $(1,0,0)$ & 2 \\
\hline & & & & & & & & & & $(0,1,0)$ & 2 \\
\hline & & & & & & & & \multirow[t]{2}{*}{$(10,0)$} & \multirow[t]{2}{*}{4} & $(1,0,0)$ & 2 \\
\hline & & & & & & & & & & $(0,1,0)$ & 2 \\
\hline & & & & \multirow[t]{2}{*}{$(001,2,00)$} & \multirow[t]{2}{*}{12} & \multirow[t]{2}{*}{$(10,2,00)$} & \multirow[t]{2}{*}{12} & \multirow[t]{2}{*}{$(10,2)$} & \multirow[t]{2}{*}{12} & $(1,0,2)$ & 6 \\
\hline & & & & & & & & & & $(0,1,2)$ & 6 \\
\hline & & \multirow[t]{6}{*}{$(100,0001)$} & \multirow[t]{6}{*}{32} & \multirow[t]{6}{*}{$(100,1,10)$} & \multirow[t]{6}{*}{32} & \multirow[t]{6}{*}{$(10,1,10)$} & \multirow[t]{6}{*}{32} & \multirow[t]{3}{*}{$(20,1)$} & \multirow[t]{3}{*}{20} & $(1,1,1)$ & 8 \\
\hline & & & & & & & & & & $(2,0,1)$ & 6 \\
\hline & & & & & & & & & & $(0,2,1)$ & 6 \\
\hline & & & & & & & & \multirow[t]{2}{*}{$(01,1)$} & \multirow[t]{2}{*}{10} & $(1,1,1)$ & 8 \\
\hline & & & & & & & & & & $(0,0,1)$ & 2 \\
\hline & & & & & & & & $(00,1)$ & 2 & $(0,0,1)$ & 2 \\
\hline \multicolumn{2}{|l|}{1 S.I. } & 2 S.I. & & 3 S.I. & & 3 S.I. & & $6 \mathrm{~s}$ & & $14 \mathrm{~s}$ & \\
\hline
\end{tabular}


11. $D_{7} \supset A_{3} \oplus D_{4} \supset A_{3} \oplus A_{1} \oplus C_{2} \supset C_{2} \oplus A_{1} \oplus C_{2} \supset A_{1} \oplus A_{1} \oplus A_{1} \oplus C_{2} \supset$

$\supset A_{1} \oplus A_{1} \oplus A_{1} \oplus A_{1} \oplus A_{1} \supset\left(A_{1}\right)_{14} \oplus A_{1} \oplus A_{1} \oplus A_{1}$.

\begin{tabular}{|c|c|c|c|c|c|c|c|c|c|c|c|c|c|}
\hline \multicolumn{2}{|l|}{$D_{7}$} & \multicolumn{2}{|l|}{$A_{3} \oplus D_{4}$} & \multicolumn{2}{|c|}{$A_{3} \oplus A_{1} \oplus C_{2}$} & \multicolumn{2}{|c|}{$C_{2} \oplus A_{1} \oplus C_{2}$} & \multicolumn{2}{|c|}{$\left(A_{1}\right)^{3} \oplus C_{2}$} & \multicolumn{2}{|l|}{$\left(A_{1}\right)^{5}$} & \multicolumn{2}{|l|}{$\left(A_{1}\right)^{4}$} \\
\hline Peso & $d$ & Peso & $d$ & Peso & $d$ & Peso & $d$ & Peso & $d$ & Peso & $d$ & Pesp & $d$ \\
\hline \multirow[t]{12}{*}{$(0000010)$} & \multirow[t]{12}{*}{64} & \multirow[t]{7}{*}{$(001,0010)$} & \multirow[t]{7}{*}{32} & \multirow[t]{5}{*}{$(001,0,01)$} & \multirow[t]{5}{*}{20} & \multirow[t]{5}{*}{$(10,0,01)$} & \multirow[t]{5}{*}{20} & \multirow[t]{3}{*}{$(1,0,0,01)$} & \multirow[t]{3}{*}{10} & \multirow[t]{2}{*}{$(1,0,0,1,1)$} & \multirow[t]{2}{*}{8} & $(2,0,0,1)$ & 6 \\
\hline & & & & & & & & & & & & $(0,0,0,1)$ & 2 \\
\hline & & & & & & & & & & $(1,0,0,0,0)$ & 2 & $(1,0,0,0)$ & 2 \\
\hline & & & & & & & & \multirow[t]{2}{*}{$(0,1,0,01)$} & \multirow[t]{2}{*}{10} & $(0,1,0,1,1)$ & 8 & $(1,1,0,1)$ & 8 \\
\hline & & & & & & & & & & $(0,1,0,0,0)$ & 2 & $(0,1,0,0)$ & 2 \\
\hline & & & & \multirow{2}{*}{$(001,2,00)$} & \multirow[t]{2}{*}{12} & \multirow{2}{*}{$(10,2,00)$} & \multirow[t]{2}{*}{12} & $(1,0,2,00)$ & 6 & $(1,0,2,0,0)$ & 6 & $(1,0,2,0)$ & 6 \\
\hline & & & & & & & & $(0,1,2,00)$ & 6 & $(0,1,2,0,0)$ & 6 & $(0,1,2,0)$ & 6 \\
\hline & & \multirow[t]{5}{*}{$(100,0001)$} & \multirow[t]{5}{*}{32} & \multirow[t]{5}{*}{$(100,1,10)$} & \multirow[t]{5}{*}{32} & \multirow[t]{5}{*}{$(10,1,10)$} & \multirow[t]{5}{*}{32} & \multirow[t]{3}{*}{$(1,0,1,10)$} & \multirow[t]{3}{*}{16} & \multirow[t]{2}{*}{$(1,0,1,1,0)$} & \multirow[t]{2}{*}{8} & $(2,0,1,0)$ & 6 \\
\hline & & & & & & & & & & & & $(0,0,1,0)$ & 2 \\
\hline & & & & & & & & & & $(1,0,1,0,1)$ & 8 & $(1,0,1,1)$ & 8 \\
\hline & & & & & & & & $(0,1,1,10)$ & 16 & $(0,1,1,1,0)$ & 8 & $(1,1,1,0)$ & 8 \\
\hline & & & & & & & & & & $(0,1,1,0,1)$ & 8 & $(0,1,1,1)$ & 8 \\
\hline 1 S.I. & & 2 S.I. & & 3 S.I. & & 3 S.I. & & 6 S.I. & & 10 S.I. & & 12 S.I. & \\
\hline
\end{tabular}

12. $D_{7} \supset A_{3} \oplus D_{4} \supset A_{3} \oplus A_{1} \oplus C_{2} \supset A_{1} \oplus A_{1} \oplus A_{1} \oplus C_{2} \supset A_{1} \oplus A_{1} \oplus A_{1} \oplus A_{1} \oplus A_{1} \supset\left(A_{1}\right)_{13} \oplus A_{1} \oplus A_{1} \oplus A_{1}$.

\begin{tabular}{|c|c|c|c|c|c|c|c|c|c|c|c|}
\hline \multicolumn{2}{|l|}{$D_{7}$} & \multicolumn{2}{|c|}{$A_{3} \oplus D_{4}$} & \multicolumn{2}{|c|}{$A_{3} \oplus A_{1} \oplus C_{2}$} & \multicolumn{2}{|c|}{$\left(A_{1}\right)^{3} \oplus C_{2}$} & \multicolumn{2}{|l|}{$\left(A_{1}\right)^{5}$} & \multicolumn{2}{|c|}{$\left(A_{1}\right)^{4}$} \\
\hline Peso & $d$ & Peso & $d$ & Peso & $d$ & Peso & $d$ & Peso & $d$ & Peso & $d$ \\
\hline \multirow[t]{8}{*}{$(0000010)$} & \multirow[t]{8}{*}{64} & \multirow[t]{4}{*}{$(001,0010)$} & \multirow[t]{4}{*}{32} & \multirow[t]{2}{*}{$(001,0,01)$} & \multirow[t]{2}{*}{20} & \multirow[t]{2}{*}{$(1,1,0,01)$} & \multirow[t]{2}{*}{20} & $(1,1,0,1,1)$ & 16 & $(1,1,1,1)$ & 16 \\
\hline & & & & & & & & $(1,1,0,0,0)$ & 4 & $(1,1,0,0)$ & 4 \\
\hline & & & & \multirow[t]{2}{*}{$(001,2,00)$} & \multirow[t]{2}{*}{12} & \multirow[t]{2}{*}{$(1,1,2,00)$} & \multirow[t]{2}{*}{12} & \multirow[t]{2}{*}{$(1,1,2,0,0)$} & \multirow[t]{2}{*}{12} & $(1,1,0,0)$ & 4 \\
\hline & & & & & & & & & & $(3,1,0,0)$ & 8 \\
\hline & & \multirow[t]{4}{*}{$(100,0001)$} & \multirow[t]{4}{*}{32} & \multirow[t]{4}{*}{$(100,1,10)$} & \multirow[t]{4}{*}{32} & \multirow[t]{4}{*}{$(1,1,1,10)$} & \multirow[t]{4}{*}{32} & \multirow[t]{2}{*}{$(1,1,1,1,0)$} & \multirow[t]{2}{*}{16} & $(0,1,1,0)$ & 4 \\
\hline & & & & & & & & & & $(2,1,1,0)$ & 12 \\
\hline & & & & & & & & \multirow[t]{2}{*}{$(1,1,1,0,1)$} & \multirow[t]{2}{*}{16} & $(0,1,0,1)$ & 4 \\
\hline & & & & & & & & & & $(2,1,0,1)$ & 12 \\
\hline \multicolumn{2}{|l|}{1 S.I. } & \multicolumn{2}{|l|}{2 S.I. } & \multicolumn{2}{|l|}{3 S.I. } & \multicolumn{2}{|l|}{3 S.I. } & \multicolumn{2}{|l|}{5 S.I. } & \multicolumn{2}{|l|}{8 S.I. } \\
\hline
\end{tabular}


13. $D_{7} \supset A_{3} \oplus D_{4} \supset A_{3} \oplus A_{1} \oplus C_{2} \supset A_{1} \oplus A_{1} \oplus A_{1} \oplus C_{2} \supset A_{1} \oplus A_{1} \oplus A_{1} \oplus A_{1} \oplus A_{1} \supset\left(A_{1}\right)_{14} \oplus A_{1} \oplus A_{1} \oplus A_{1}$.

\begin{tabular}{|c|c|c|c|c|c|c|c|c|c|c|c|}
\hline \multicolumn{2}{|l|}{$D_{7}$} & \multicolumn{2}{|c|}{$A_{3} \oplus D_{4}$} & \multicolumn{2}{|c|}{$A_{3} \oplus A_{1} \oplus C_{2}$} & \multicolumn{2}{|c|}{$\left(A_{1}\right)^{3} \oplus C_{2}$} & \multicolumn{2}{|l|}{$\left(A_{1}\right)^{5}$} & \multicolumn{2}{|c|}{$\left(A_{1}\right)^{4}$} \\
\hline Peso & $d$ & Peso & $d$ & Peso & $d$ & Peso & $d$ & Peso & $d$ & Peso & $d$ \\
\hline \multirow[t]{7}{*}{ (0000010) } & \multirow[t]{7}{*}{64} & \multirow[t]{4}{*}{$(001,0010)$} & \multirow[t]{4}{*}{32} & \multirow[t]{3}{*}{$(001,0,01)$} & \multirow[t]{3}{*}{20} & \multirow[t]{3}{*}{$(1,1,0,01)$} & \multirow[t]{3}{*}{20} & \multirow[t]{2}{*}{$(1,1,0,1,1)$} & \multirow[t]{2}{*}{16} & $(0,1,0,1)$ & 4 \\
\hline & & & & & & & & & & $(2,1,0,1)$ & 12 \\
\hline & & & & & & & & $(1,1,0,0,0)$ & 4 & $(1,1,0,0)$ & 4 \\
\hline & & & & $(001,2,00)$ & 12 & $(1,1,2,00)$ & 12 & $(1,1,2,0,0)$ & 12 & $(1,1,2,0)$ & 12 \\
\hline & & \multirow[t]{3}{*}{$(100,0001)$} & \multirow[t]{3}{*}{32} & \multirow[t]{3}{*}{$(100,1,10)$} & \multirow[t]{3}{*}{32} & \multirow[t]{3}{*}{$(1,1,1,10)$} & \multirow[t]{3}{*}{32} & \multirow[t]{2}{*}{$(1,1,1,1,0)$} & \multirow[t]{2}{*}{16} & $(0,1,1,0)$ & 4 \\
\hline & & & & & & & & & & $(2,1,1,0)$ & 12 \\
\hline & & & & & & & & $(1,1,1,0,1)$ & 16 & $(1,1,1,1)$ & 16 \\
\hline \multicolumn{2}{|l|}{1 S.I. } & \multicolumn{2}{|l|}{2 S.I. } & \multicolumn{2}{|l|}{3 S.I. } & \multicolumn{2}{|l|}{3 S.I. } & \multicolumn{2}{|l|}{5 S.I. } & \multicolumn{2}{|l|}{7 S.I. } \\
\hline
\end{tabular}

14. $D_{7} \supset A_{3} \oplus D_{4} \supset A_{3} \oplus A_{1} \oplus C_{2} \supset A_{1} \oplus A_{1} \oplus A_{1} \oplus C_{2} \supset A_{1} \oplus A_{1} \oplus A_{1} \oplus A_{1} \oplus A_{1} \supset\left(A_{1}\right)_{45} \oplus A_{1} \oplus A_{1} \oplus A_{1}$.

\begin{tabular}{|c|c|c|c|c|c|c|c|c|c|c|c|}
\hline \multicolumn{2}{|l|}{$D_{7}$} & \multicolumn{2}{|c|}{$A_{3} \oplus D_{4}$} & \multicolumn{2}{|c|}{$A_{3} \oplus A_{1} \oplus C_{2}$} & \multicolumn{2}{|c|}{$\left(A_{1}\right)^{3} \oplus C_{2}$} & \multicolumn{2}{|l|}{$\left(A_{1}\right)^{5}$} & \multicolumn{2}{|l|}{$\left(A_{1}\right)^{4}$} \\
\hline Peso & $d$ & Peso & $d$ & Peso & $d$ & Peso & $d$ & Peso & $d$ & Peso & $d$ \\
\hline \multirow[t]{6}{*}{$(0000010)$} & \multirow[t]{6}{*}{64} & \multirow[t]{4}{*}{$(001,0010)$} & \multirow[t]{4}{*}{32} & \multirow[t]{3}{*}{$(001,0,01)$} & \multirow[t]{3}{*}{20} & \multirow[t]{3}{*}{$(1,1,0,01)$} & \multirow[t]{3}{*}{20} & \multirow[t]{2}{*}{$(1,1,0,1,1)$} & \multirow[t]{2}{*}{16} & $(0,1,1,0)$ & 4 \\
\hline & & & & & & & & & & $(2,1,1,0)$ & 12 \\
\hline & & & & & & & & $(1,1,0,0,0)$ & 4 & $(0,1,1,0)$ & 4 \\
\hline & & & & $(001,2,00)$ & 12 & $(1,1,2,00)$ & 12 & $(1,1,2,0,0)$ & 12 & $(0,1,1,2)$ & 12 \\
\hline & & \multirow[t]{2}{*}{$(100,0001)$} & \multirow[t]{2}{*}{32} & \multirow[t]{2}{*}{$(100,1,10)$} & \multirow[t]{2}{*}{32} & \multirow[t]{2}{*}{$(1,1,1,10)$} & \multirow[t]{2}{*}{32} & $(1,1,1,1,0)$ & 16 & $(1,1,1,1)$ & 16 \\
\hline & & & & & & & & $(1,1,1,0,1)$ & 16 & $(1,1,1,1)$ & 16 \\
\hline \multicolumn{2}{|l|}{1 S.I. } & \multicolumn{2}{|l|}{2 S.I. } & \multicolumn{2}{|l|}{3 S.I. } & \multicolumn{2}{|l|}{3 S.I. } & \multicolumn{2}{|l|}{5 S.I. } & \multicolumn{2}{|l|}{6 S.I. } \\
\hline
\end{tabular}


15. $D_{7} \supset A_{3} \oplus D_{4} \supset A_{3} \oplus A_{1} \oplus A_{1} \oplus A_{1} \oplus A_{1} \supset A_{2} \oplus A_{1} \oplus A_{1} \oplus A_{1} \oplus A_{1} \supset$

$\supset \tilde{A}_{1} \oplus A_{1} \oplus A_{1} \oplus A_{1} \oplus A_{1} \supset\left(A_{1}\right)_{12} \oplus A_{1} \oplus A_{1} \oplus A_{1}$.

\begin{tabular}{|c|c|c|c|c|c|c|c|c|c|c|c|}
\hline \multicolumn{2}{|l|}{$D_{7}$} & \multicolumn{2}{|c|}{$A_{3} \oplus D_{4}$} & \multicolumn{2}{|c|}{$A_{3} \oplus\left(A_{1}\right)^{4}$} & \multicolumn{2}{|c|}{$A_{2} \oplus\left(A_{1}\right)^{4}$} & \multicolumn{2}{|l|}{$\left(A_{1}\right)^{5}$} & \multicolumn{2}{|c|}{$\left(A_{1}\right)^{4}$} \\
\hline Peso & $d$ & Peso & $d$ & Peso & $d$ & Peso & $d$ & Peso & $d$ & Peso & $d$ \\
\hline \multirow[t]{10}{*}{ (0000010) } & \multirow[t]{10}{*}{64} & \multirow[t]{5}{*}{$(001,0010)$} & \multirow[t]{5}{*}{32} & \multirow[t]{2}{*}{$(001,0,1,1,0)$} & \multirow[t]{2}{*}{16} & $(01,0,1,1,0)$ & 12 & $(2,0,1,1,0)$ & 12 & $(2,1,1,0)$ & 12 \\
\hline & & & & & & $(00,0,1,1,0)$ & 4 & $(0,0,1,1,0)$ & 4 & $(0,1,1,0)$ & 4 \\
\hline & & & & \multirow[t]{3}{*}{$(001,1,0,0,1)$} & \multirow[t]{3}{*}{16} & \multirow[t]{2}{*}{$(01,1,0,0,1)$} & \multirow[t]{2}{*}{12} & \multirow[t]{2}{*}{$(2,1,0,0,1)$} & \multirow[t]{2}{*}{12} & $(1,0,0,1)$ & 4 \\
\hline & & & & & & & & & & $(3,0,0,1)$ & 8 \\
\hline & & & & & & $(00,1,0,0,1)$ & 4 & $(0,1,0,0,1)$ & 4 & $(1,0,0,1)$ & 4 \\
\hline & & \multirow[t]{5}{*}{$(100,0001)$} & \multirow[t]{5}{*}{32} & \multirow[t]{2}{*}{$(100,0,1,1,0)$} & \multirow[t]{2}{*}{16} & $(10,0,1,0,1)$ & 12 & $(2,0,1,0,1)$ & 12 & $(2,1,0,1)$ & 12 \\
\hline & & & & & & $(00,0,1,0,1)$ & 4 & $(0,0,1,0,1)$ & 4 & $(0,1,0,1)$ & 4 \\
\hline & & & & \multirow[t]{3}{*}{$(100,1,0,1,0)$} & \multirow[t]{3}{*}{16} & \multirow[t]{2}{*}{$(10,1,0,1,0)$} & \multirow[t]{2}{*}{12} & \multirow[t]{2}{*}{$(2,1,0,1,0)$} & \multirow[t]{2}{*}{12} & $(1,0,1,0)$ & 4 \\
\hline & & & & & & & & & & $(3,0,1,0)$ & 8 \\
\hline & & & & & & $(00,1,0,1,0)$ & 4 & $(0,1,0,1,0)$ & 4 & $(1,0,1,0)$ & 4 \\
\hline \multicolumn{2}{|l|}{1 S.I. } & \multicolumn{2}{|l|}{2 S.I. } & \multicolumn{2}{|l|}{4 S.I. } & \multicolumn{2}{|l|}{8 S.I. } & \multicolumn{2}{|l|}{8 S.I. } & \multicolumn{2}{|l|}{10 S.I. } \\
\hline
\end{tabular}


16. $D_{7} \supset A_{3} \oplus D_{4} \supset A_{3} \oplus A_{1} \oplus C_{2} \supset A_{2} \oplus A_{1} \oplus C_{2} \supset A_{1} \oplus A_{1} \oplus C_{2} \supset A_{1} \oplus A_{1} \oplus A_{1} \oplus A_{1} \supset\left(A_{1}\right)_{13} \oplus A_{1} \oplus A_{1}$.

\begin{tabular}{|c|c|c|c|c|c|c|c|c|c|c|c|c|c|}
\hline \multicolumn{2}{|l|}{$D_{7}$} & \multicolumn{2}{|l|}{$A_{3} \oplus D_{4}$} & \multicolumn{2}{|c|}{$A_{3} \oplus A_{1} \oplus C_{2}$} & \multicolumn{2}{|c|}{$A_{2} \oplus A_{1} \oplus C_{2}$} & \multicolumn{2}{|c|}{$\left(A_{1}\right)^{3} \oplus C_{2}$} & \multicolumn{2}{|l|}{$\left(A_{1}\right)^{4}$} & \multicolumn{2}{|c|}{$\left(A_{1}\right)^{3}$} \\
\hline Peso & $d$ & Peso & $d$ & Peso & $d$ & Peso & $d$ & Peso & $d$ & Peso & $d$ & Peso & $d$ \\
\hline \multirow[t]{17}{*}{$(0000010)$} & \multirow[t]{17}{*}{64} & \multirow[t]{10}{*}{$(001,0010)$} & \multirow[t]{10}{*}{32} & \multirow[t]{7}{*}{$(001,0,01)$} & \multirow[t]{7}{*}{20} & \multirow[t]{5}{*}{$(01,0,01)$} & \multirow[t]{5}{*}{15} & \multirow[t]{3}{*}{$(1,0,01)$} & \multirow[t]{3}{*}{10} & \multirow[t]{2}{*}{$(1,0,1,1)$} & \multirow[t]{2}{*}{8} & $(0,0,1)$ & 2 \\
\hline & & & & & & & & & & & & $(2,0,1)$ & 6 \\
\hline & & & & & & & & & & $(1,0,0,0)$ & 2 & $(1,0,0)$ & 2 \\
\hline & & & & & & & & \multirow[t]{2}{*}{$(0,0,01)$} & \multirow[t]{2}{*}{5} & $(0,0,1,1)$ & 4 & $(1,0,1)$ & 4 \\
\hline & & & & & & & & & & $(0,0,0,0)$ & 1 & $(0,0,0)$ & 1 \\
\hline & & & & & & \multirow[t]{2}{*}{$(00,0,01)$} & \multirow[t]{2}{*}{5} & \multirow{2}{*}{$(0,0,01)$} & \multirow[t]{2}{*}{5} & $(0,0,1,1)$ & 4 & $(0,1,1)$ & 4 \\
\hline & & & & & & & & & & $(0,0,0,0)$ & 1 & $(0,0,0)$ & 1 \\
\hline & & & & \multirow[t]{3}{*}{$(001,2,00)$} & \multirow[t]{3}{*}{12} & \multirow[t]{2}{*}{$(01,2,00)$} & \multirow[t]{2}{*}{9} & $(1,2,00)$ & 6 & $(1,2,0,0)$ & 6 & $(1,2,0)$ & 6 \\
\hline & & & & & & & & $(0,2,00)$ & 3 & $(0,2,0,0)$ & 3 & $(0,2,0)$ & 3 \\
\hline & & & & & & $(00,2,00)$ & 3 & $(0,2,00)$ & 3 & $(0,2,0,0)$ & 3 & $(0,2,0)$ & 3 \\
\hline & & \multirow[t]{7}{*}{$(100,0001)$} & \multirow[t]{7}{*}{32} & \multirow[t]{7}{*}{$(100,1,10)$} & 32 & $(10,1,10)$ & 24 & $(1,1,10)$ & 16 & $(1,1,1,0)$ & 8 & $(0,1,0)$ & 2 \\
\hline & & & & & & & & & & & & $(2,1,0)$ & 6 \\
\hline & & & & & & & & & & $(1,1,0,1)$ & 8 & $(1,1,1)$ & 8 \\
\hline & & & & & & & & $(0,1,10)$ & 8 & $(0,1,1,0)$ & 4 & $(1,1,0)$ & 4 \\
\hline & & & & & & & & & & $(0,1,0,1)$ & 4 & $(0,1,1)$ & 4 \\
\hline & & & & & & $(00,1,10)$ & 8 & $(0,1,10)$ & 8 & $(0,1,1,0)$ & 4 & $(1,1,0)$ & 4 \\
\hline & & & & & & & & & & $(0,1,0,1)$ & 4 & $(0,1,1)$ & 4 \\
\hline 1 S.I. & & 2 S.I. & & 3 S.I. & & 6 S.I. & & 9 S.I. & & 15 S.I. & & 17 S.I. & \\
\hline
\end{tabular}


17. $D_{7} \supset A_{3} \oplus D_{4} \supset A_{3} \oplus A_{1} \oplus C_{2} \supset A_{2} \oplus A_{1} \oplus C_{2} \supset \tilde{A}_{1} \oplus A_{1} \oplus C_{2} \supset A_{1} \oplus A_{1} \oplus A_{1} \oplus A_{1} \supset\left(A_{1}\right)_{13} \oplus A_{1} \oplus A_{1}$.

\begin{tabular}{|c|c|c|c|c|c|c|c|c|c|c|c|c|c|}
\hline \multicolumn{2}{|l|}{$D_{7}$} & \multicolumn{2}{|l|}{$A_{3} \oplus D_{4}$} & \multicolumn{2}{|c|}{$A_{3} \oplus A_{1} \oplus C_{2}$} & \multicolumn{2}{|c|}{$A_{2} \oplus A_{1} \oplus C_{2}$} & \multicolumn{2}{|c|}{$\left(A_{1}\right)^{2} \oplus C_{2}$} & \multicolumn{2}{|l|}{$\left(A_{1}\right)^{4}$} & \multicolumn{2}{|c|}{$\left(A_{1}\right)^{3}$} \\
\hline Peso & $d$ & Peso & $d$ & Peso & $d$ & Peso & $d$ & Peso & $d$ & Peso & $d$ & Peso & $d$ \\
\hline \multirow[t]{12}{*}{$(0000010)$} & \multirow[t]{12}{*}{64} & \multirow[t]{7}{*}{$(001,0010)$} & \multirow[t]{7}{*}{32} & \multirow[t]{5}{*}{$(001,0,01)$} & \multirow[t]{5}{*}{20} & \multirow[t]{3}{*}{$(01,0,01)$} & \multirow[t]{3}{*}{15} & \multirow[t]{2}{*}{$(2,0,01)$} & \multirow[t]{2}{*}{12} & \multirow[t]{2}{*}{$(2,0,1,1)$} & \multirow[t]{2}{*}{12} & $(1,0,1)$ & 4 \\
\hline & & & & & & & & & & & & $(3,0,1)$ & 8 \\
\hline & & & & & & & & $(2,0,00)$ & 3 & $(2,0,0,0)$ & 3 & $(2,0,0)$ & 3 \\
\hline & & & & & & \multirow[t]{2}{*}{$(00,0,01)$} & \multirow[t]{2}{*}{5} & \multirow[t]{2}{*}{$(0,0,01)$} & \multirow[t]{2}{*}{5} & $(0,0,1,1)$ & 4 & $(1,0,1)$ & 4 \\
\hline & & & & & & & & & & $(0,0,0,0)$ & 1 & $(0,0,0)$ & 1 \\
\hline & & & & \multirow[t]{2}{*}{$(001,2,00)$} & \multirow[t]{2}{*}{12} & $(01,2,00)$ & 9 & $(2,2,00)$ & 9 & $(2,2,0,0)$ & 9 & $(2,2,0)$ & 9 \\
\hline & & & & & & $(00,2,00)$ & 3 & $(0,2,00)$ & 3 & $(0,2,0,0)$ & 3 & $(0,2,0)$ & 3 \\
\hline & & \multirow[t]{5}{*}{$(100,0001)$} & \multirow[t]{5}{*}{32} & \multirow[t]{5}{*}{$(100,1,10)$} & \multirow[t]{5}{*}{32} & \multirow[t]{3}{*}{$(10,1,10)$} & \multirow[t]{3}{*}{24} & \multirow[t]{3}{*}{$(2,1,10)$} & \multirow[t]{3}{*}{24} & \multirow[t]{2}{*}{$(2,1,1,0)$} & \multirow[t]{2}{*}{12} & $(1,1,0)$ & 4 \\
\hline & & & & & & & & & & & & $(3,1,0)$ & 8 \\
\hline & & & & & & & & & & $(2,1,0,1)$ & 12 & $(2,1,1)$ & 12 \\
\hline & & & & & & $(00,1,10)$ & 8 & $(0,1,10)$ & 8 & $(0,1,1,0)$ & 4 & $(1,1,0)$ & 4 \\
\hline & & & & & & & & & & $(0,1,0,1)$ & 4 & $(0,1,1)$ & 4 \\
\hline 1 S.I. & & 2 S.I. & & 3 S.I. & & 6 S.I. & & 7 S.I. & & 10 S.I. & & $12 \mathrm{~S} . \mathrm{I}$ & \\
\hline
\end{tabular}

18. $D_{7} \supset A_{3} \oplus D_{4} \supset A_{3} \oplus A_{1} \oplus C_{2} \supset C_{2} \oplus A_{1} \oplus C_{2} \supset A_{1} \oplus A_{1} \oplus A_{1} \oplus C_{2} \supset A_{1} \oplus A_{1} \oplus A_{1} \oplus A_{1} \supset\left(A_{1}\right)_{14} \oplus A_{1} \oplus A_{1}$.

\begin{tabular}{|c|c|c|c|c|c|c|c|c|c|c|c|c|c|}
\hline \multicolumn{2}{|l|}{$D_{7}$} & \multicolumn{2}{|l|}{$A_{3} \oplus D_{4}$} & \multicolumn{2}{|c|}{$A_{3} \oplus A_{1} \oplus C_{2}$} & \multicolumn{2}{|c|}{$C_{2} \oplus A_{1} \oplus C_{2}$} & \multicolumn{2}{|c|}{$\left(A_{1}\right)^{3} \oplus C_{2}$} & \multicolumn{2}{|l|}{$\left(A_{1}\right)^{4}$} & \multicolumn{2}{|c|}{$\left(A_{1}\right)^{3}$} \\
\hline Peso & $d$ & Peso & $d$ & Peso & $d$ & Peso & $d$ & Peso & $d$ & Peso & $d$ & Peso & $d$ \\
\hline \multirow[t]{8}{*}{$(0000010)$} & \multirow[t]{8}{*}{64} & \multirow[t]{5}{*}{$(001,0010)$} & \multirow[t]{5}{*}{32} & \multirow[t]{3}{*}{$(001,0,01)$} & \multirow[t]{3}{*}{20} & \multirow[t]{3}{*}{$(10,0,01)$} & \multirow[t]{3}{*}{20} & $(1,0,0,01)$ & \multirow[t]{2}{*}{10} & \multirow[t]{2}{*}{$(1,0,0,4)$} & \multirow[t]{2}{*}{10} & $(3,0,0)$ & 4 \\
\hline & & & & & & & & & & & & $(5,0,0)$ & 6 \\
\hline & & & & & & & & $(0,1,0,01)$ & 10 & $(0,1,0,4)$ & 10 & $(4,1,0)$ & 10 \\
\hline & & & & \multirow[t]{2}{*}{$(001,2,00)$} & \multirow[t]{2}{*}{12} & \multirow[t]{2}{*}{$(10,2,00)$} & \multirow[t]{2}{*}{12} & $(1,0,2,00)$ & 6 & $(1,0,2,0)$ & 6 & $(1,0,2)$ & 6 \\
\hline & & & & & & & & $(0,1,2,00)$ & 6 & $(0,1,2,0)$ & 6 & $(0,1,2)$ & 6 \\
\hline & & \multirow[t]{3}{*}{$(100,0001)$} & \multirow[t]{3}{*}{32} & \multirow[t]{3}{*}{$(100,1,10)$} & \multirow[t]{3}{*}{32} & \multirow[t]{3}{*}{$(10,1,10)$} & \multirow[t]{3}{*}{32} & $(1,0,1,10)$ & \multirow[t]{2}{*}{16} & \multirow[t]{2}{*}{$(1,0,1,3)$} & \multirow[t]{2}{*}{16} & $(2,0,1)$ & 6 \\
\hline & & & & & & & & & & & & $(4,0,1)$ & 10 \\
\hline & & & & & & & & $(0,1,1,10)$ & 16 & $(0,1,1,3)$ & 16 & $(3,1,1)$ & 16 \\
\hline \multicolumn{2}{|l|}{1 S.I. } & \multicolumn{2}{|l|}{2 S.I. } & \multicolumn{2}{|l|}{3 S.I. } & \multicolumn{2}{|l|}{3 S.I. } & \multicolumn{2}{|l|}{6 S.I. } & \multicolumn{2}{|l|}{6 S.I. } & 8 S.I. & \\
\hline
\end{tabular}


19. $D_{7} \supset A_{3} \oplus D_{4} \supset A_{3} \oplus A_{1} \oplus C_{2} \supset C_{2} \oplus A_{1} \oplus C_{2} \supset A_{1} \oplus A_{1} \oplus C_{2} \supset A_{1} \oplus A_{1} \oplus A_{1} \oplus A_{1} \supset\left(A_{1}\right)_{13} \oplus A_{1} \oplus A_{1}$.

\begin{tabular}{|c|c|c|c|c|c|c|c|c|c|c|c|c|c|}
\hline \multicolumn{2}{|l|}{$D_{7}$} & \multicolumn{2}{|l|}{$A_{3} \oplus D_{4}$} & \multicolumn{2}{|c|}{$A_{3} \oplus A_{1} \oplus C_{2}$} & \multicolumn{2}{|c|}{$C_{2} \oplus A_{1} \oplus C_{2}$} & \multicolumn{2}{|c|}{$\left(A_{1}\right)^{2} \oplus C_{2}$} & \multicolumn{2}{|l|}{$\left(A_{1}\right)^{4}$} & \multicolumn{2}{|c|}{$\left(A_{1}\right)^{3}$} \\
\hline Peso & $d$ & Peso & $d$ & Peso & $d$ & Peso & $d$ & Peso & $d$ & Peso & $d$ & Peso & $d$ \\
\hline \multirow[t]{7}{*}{ (0000010) } & \multirow[t]{7}{*}{64} & \multirow[t]{4}{*}{$(001,0010)$} & \multirow[t]{4}{*}{32} & \multirow[t]{3}{*}{$(001,0,01)$} & \multirow[t]{3}{*}{20} & \multirow[t]{3}{*}{$(10,0,01)$} & \multirow[t]{3}{*}{20} & \multirow[t]{3}{*}{$(3,0,01)$} & \multirow[t]{3}{*}{20} & \multirow[t]{2}{*}{$(3,0,1,1)$} & \multirow[t]{2}{*}{16} & $(2,0,1)$ & 6 \\
\hline & & & & & & & & & & & & $(4,0,1)$ & 10 \\
\hline & & & & & & & & & & $(3,0,0,0)$ & 4 & $(3,0,0)$ & 4 \\
\hline & & & & $(001,2,00)$ & 12 & $(10,2,00)$ & 12 & $(3,2,00)$ & 12 & $(3,2,0,0)$ & 12 & $(3,2,0)$ & 12 \\
\hline & & \multirow[t]{3}{*}{$(100,0001)$} & \multirow[t]{3}{*}{32} & \multirow[t]{3}{*}{$(100,1,10)$} & \multirow[t]{3}{*}{32} & \multirow[t]{3}{*}{$(10,1,10)$} & \multirow[t]{3}{*}{32} & \multirow[t]{3}{*}{$(3,1,10)$} & \multirow[t]{3}{*}{16} & \multirow[t]{2}{*}{$(3,1,1,0)$} & \multirow[t]{2}{*}{16} & $(2,1,0)$ & 6 \\
\hline & & & & & & & & & & & & $(4,1,0)$ & 10 \\
\hline & & & & & & & & & & $(3,1,0,1)$ & 16 & $(3,1,1)$ & 16 \\
\hline \multicolumn{2}{|l|}{1 S.I. } & \multicolumn{2}{|l|}{2 S.I. } & \multicolumn{2}{|l|}{3 S.I. } & \multicolumn{2}{|l|}{3 S.I. } & \multicolumn{2}{|l|}{3 S.I. } & \multicolumn{2}{|l|}{5 S.I. } & \multicolumn{2}{|l|}{7 S.I. } \\
\hline
\end{tabular}

20. $D_{7} \supset A_{3} \oplus D_{4} \supset A_{3} \oplus A_{1} \oplus C_{2} \supset C_{2} \oplus A_{1} \oplus C_{2} \supset A_{1} \oplus A_{1} \oplus C_{2} \supset A_{1} \oplus A_{1} \oplus A_{1} \oplus A_{1} \supset\left(A_{1}\right)_{34} \oplus A_{1} \oplus A_{1}$.

\begin{tabular}{|c|c|c|c|c|c|c|c|c|c|c|c|c|c|}
\hline \multicolumn{2}{|l|}{$D_{7}$} & \multicolumn{2}{|l|}{$A_{3} \oplus D_{4}$} & \multicolumn{2}{|c|}{$A_{3} \oplus A_{1} \oplus C_{2}$} & \multicolumn{2}{|c|}{$C_{2} \oplus A_{1} \oplus C_{2}$} & \multicolumn{2}{|c|}{$\left(A_{1}\right)^{2} \oplus C_{2}$} & \multicolumn{2}{|l|}{$\left(A_{1}\right)^{4}$} & \multicolumn{2}{|c|}{$\left(A_{1}\right)^{3}$} \\
\hline Peso & $d$ & Peso & $d$ & Peso & $d$ & Peso & $d$ & Peso & $d$ & Peso & $d$ & Peso & $d$ \\
\hline \multirow[t]{6}{*}{ (0000010) } & \multirow[t]{6}{*}{64} & \multirow[t]{4}{*}{$(001,0010)$} & \multirow[t]{4}{*}{32} & \multirow[t]{3}{*}{$(001,0,01)$} & \multirow[t]{3}{*}{20} & \multirow[t]{3}{*}{$(10,0,01)$} & \multirow[t]{3}{*}{20} & \multirow[t]{3}{*}{$(3,0,01)$} & \multirow[t]{3}{*}{20} & \multirow[t]{2}{*}{$(3,0,1,1)$} & \multirow[t]{2}{*}{16} & $(0,3,0)$ & 4 \\
\hline & & & & & & & & & & & & $(2,3,0)$ & 12 \\
\hline & & & & & & & & & & $(3,0,0,0)$ & 4 & $(0,3,0)$ & 4 \\
\hline & & & & $(001,2,00)$ & 12 & $(10,2,00)$ & 12 & $(3,2,00)$ & 12 & $(3,2,0,0)$ & 12 & $(0,3,2)$ & 12 \\
\hline & & \multirow[t]{2}{*}{$(100,0001)$} & \multirow[t]{2}{*}{32} & \multirow[t]{2}{*}{$(100,1,10)$} & \multirow[t]{2}{*}{32} & \multirow[t]{2}{*}{$(10,1,10)$} & \multirow[t]{2}{*}{32} & \multirow[t]{2}{*}{$(3,1,10)$} & \multirow[t]{2}{*}{16} & $(3,1,1,0)$ & 16 & $(1,3,1)$ & 16 \\
\hline & & & & & & & & & & $(3,1,0,1)$ & 16 & $(1,3,1)$ & 16 \\
\hline \multicolumn{2}{|l|}{1 S.I. } & \multicolumn{2}{|l|}{2 S.I. } & \multicolumn{2}{|l|}{3 S.I. } & \multicolumn{2}{|l|}{3 S.I. } & \multicolumn{2}{|l|}{3 S.I. } & \multicolumn{2}{|l|}{5 S.I. } & \multicolumn{2}{|l|}{6 S.I. } \\
\hline
\end{tabular}


21. $D_{7} \supset A_{3} \oplus D_{4} \supset A_{3} \oplus A_{1} \oplus C_{2} \supset A_{1} \oplus A_{1} \oplus A_{1} \oplus C_{2} \supset A_{1} \oplus A_{1} \oplus A_{1} \oplus A_{1} \supset\left(A_{1}\right)_{13} \oplus A_{1} \oplus A_{1}$.

\begin{tabular}{|c|c|c|c|c|c|c|c|c|c|c|c|}
\hline \multicolumn{2}{|l|}{$D_{7}$} & \multicolumn{2}{|c|}{$A_{3} \oplus D_{4}$} & \multicolumn{2}{|c|}{$A_{3} \oplus A_{1} \oplus C_{2}$} & \multicolumn{2}{|c|}{$\left(A_{1}\right)^{3} \oplus C_{2}$} & \multicolumn{2}{|c|}{$\left(A_{1}\right)^{4}$} & \multicolumn{2}{|c|}{$\left(A_{1}\right)^{3}$} \\
\hline Peso & $d$ & Peso & $d$ & Peso & $d$ & Peso & $d$ & Peso & $d$ & Peso & $d$ \\
\hline \multirow[t]{5}{*}{ (0000010) } & \multirow[t]{5}{*}{64} & \multirow[t]{3}{*}{$(001,0010)$} & \multirow[t]{3}{*}{32} & $(001,0,01)$ & 20 & $(1,1,0,01)$ & 20 & $(1,1,0,4)$ & 20 & $(1,1,4)$ & 20 \\
\hline & & & & \multirow[t]{2}{*}{$(001,2,00)$} & \multirow[t]{2}{*}{12} & \multirow[t]{2}{*}{$(1,1,2,00)$} & \multirow[t]{2}{*}{12} & \multirow[t]{2}{*}{$(1,1,2,0)$} & \multirow[t]{2}{*}{12} & $(1,1,0)$ & 4 \\
\hline & & & & & & & & & & $(3,1,0)$ & 8 \\
\hline & & \multirow[t]{2}{*}{$(100,0001)$} & \multirow[t]{2}{*}{32} & \multirow[t]{2}{*}{$(100,1,10)$} & \multirow[t]{2}{*}{32} & \multirow[t]{2}{*}{$(1,1,1,10)$} & \multirow[t]{2}{*}{32} & \multirow[t]{2}{*}{$(1,1,1,3)$} & \multirow[t]{2}{*}{32} & $(0,1,3)$ & 8 \\
\hline & & & & & & & & & & $(2,1,3)$ & 24 \\
\hline \multicolumn{2}{|l|}{1 S.I. } & \multicolumn{2}{|l|}{2 S.I. } & \multicolumn{2}{|l|}{3 S.I. } & \multicolumn{2}{|l|}{3 S.I. } & \multicolumn{2}{|l|}{3 S.I. } & \multicolumn{2}{|c|}{5 S.I. } \\
\hline
\end{tabular}

22. $D_{7} \supset B_{3} \oplus B_{3} \supset B_{3} \oplus G_{2} \supset G_{2} \oplus G_{2} \supset G_{2} \oplus A_{1} \oplus A_{1} \supset A_{1} \oplus A_{1} \oplus A_{1} \oplus A_{1} \supset\left(A_{1}\right)_{13} \oplus A_{1} \oplus A_{1}$.

\begin{tabular}{|c|c|c|c|c|c|c|c|c|c|c|c|c|c|}
\hline \multicolumn{2}{|l|}{$D_{7}$} & \multicolumn{2}{|c|}{$B_{3} \oplus B_{3}$} & \multicolumn{2}{|c|}{$B_{3} \oplus G_{2}$} & \multicolumn{2}{|c|}{$G_{2} \oplus G_{2}$} & \multicolumn{2}{|c|}{$G_{2} \oplus\left(A_{1}\right)^{2}$} & \multicolumn{2}{|l|}{$\left(A_{1}\right)^{4}$} & \multicolumn{2}{|c|}{$\left(A_{1}\right)^{3}$} \\
\hline Peso & $d$ & Peso & $d$ & Peso & $d$ & Peso & $d$ & Peso & $d$ & Peso & $d$ & Peso & $d$ \\
\hline \multirow[t]{10}{*}{$(0000010)$} & \multirow[t]{10}{*}{64} & \multirow[t]{10}{*}{$(001,001)$} & \multirow[t]{10}{*}{64} & \multirow[t]{7}{*}{$(001,01)$} & \multirow[t]{7}{*}{56} & \multirow[t]{5}{*}{$(01,01)$} & \multirow[t]{5}{*}{49} & \multirow[t]{3}{*}{$(01,1,1)$} & \multirow[t]{3}{*}{28} & \multirow[t]{2}{*}{$(1,1,1,1)$} & \multirow[t]{2}{*}{16} & $(0,1,1)$ & 4 \\
\hline & & & & & & & & & & & & $(2,1,1)$ & 12 \\
\hline & & & & & & & & & & $(0,2,1,1)$ & 12 & $(1,2,1)$ & 12 \\
\hline & & & & & & & & \multirow[t]{2}{*}{$(01,0,2)$} & \multirow[t]{2}{*}{21} & $(1,1,0,2)$ & 12 & $(1,1,2)$ & 12 \\
\hline & & & & & & & & & & $(0,2,0,2)$ & 9 & $(0,2,2)$ & 9 \\
\hline & & & & & & \multirow[t]{2}{*}{$(00,01)$} & \multirow[t]{2}{*}{7} & $(00,1,1)$ & 4 & $(0,0,1,1)$ & 4 & $(1,0,1)$ & 4 \\
\hline & & & & & & & & $(00,0,2)$ & 3 & $(0,0,0,2)$ & 3 & $(0,0,2)$ & 3 \\
\hline & & & & \multirow[t]{3}{*}{$(001,00)$} & \multirow[t]{3}{*}{8} & \multirow[t]{2}{*}{$(01,00)$} & \multirow[t]{2}{*}{7} & \multirow{2}{*}{$(01,0,0)$} & \multirow[t]{2}{*}{7} & $(1,1,0,0)$ & 4 & $(1,1,0)$ & 4 \\
\hline & & & & & & & & & & $(0,2,0,0)$ & 3 & $(0,2,0)$ & 3 \\
\hline & & & & & & $(00,00)$ & 1 & $(00,0,0)$ & 1 & $(0,0,0,0)$ & 1 & $(0,0,0)$ & 1 \\
\hline \multicolumn{2}{|l|}{1 S.I. } & \multicolumn{2}{|l|}{1 S.I. } & \multicolumn{2}{|l|}{2 S.I. } & \multicolumn{2}{|c|}{4 S.I. } & \multicolumn{2}{|c|}{6 S.I. } & \multicolumn{2}{|l|}{9 S.I. } & \multicolumn{2}{|c|}{10 S.I. } \\
\hline
\end{tabular}


23. $D_{7} \supset B_{3} \oplus B_{3} \supset B_{3} \oplus G_{2} \supset G_{2} \oplus G_{2} \supset G_{2} \oplus A_{1} \oplus A_{1} \supset A_{1} \oplus A_{1} \oplus A_{1} \oplus A_{1} \supset\left(A_{1}\right)_{14} \oplus A_{1} \oplus A_{1}$.

\begin{tabular}{|c|c|c|c|c|c|c|c|c|c|c|c|c|c|}
\hline \multicolumn{2}{|l|}{$D_{7}$} & \multicolumn{2}{|c|}{$B_{3} \oplus B_{3}$} & \multicolumn{2}{|c|}{$B_{3} \oplus G_{2}$} & \multicolumn{2}{|c|}{$G_{2} \oplus G_{2}$} & \multicolumn{2}{|c|}{$G_{2} \oplus\left(A_{1}\right)^{2}$} & \multicolumn{2}{|c|}{$\left(A_{1}\right)^{4}$} & \multicolumn{2}{|c|}{$\left(A_{1}\right)^{3}$} \\
\hline Peso & $d$ & Peso & $d$ & Peso & $d$ & Peso & $d$ & Peso & $d$ & Peso & $d$ & Peso & $d$ \\
\hline \multirow[t]{11}{*}{$(0000010)$} & \multirow[t]{11}{*}{64} & \multirow[t]{11}{*}{$(001,001)$} & \multirow[t]{11}{*}{64} & \multirow[t]{8}{*}{$(001,01)$} & \multirow[t]{8}{*}{56} & \multirow[t]{6}{*}{$(01,01)$} & \multirow[t]{6}{*}{49} & \multirow[t]{3}{*}{$(01,1,1)$} & \multirow[t]{3}{*}{28} & \multirow[t]{2}{*}{$(1,1,1,1)$} & \multirow[t]{2}{*}{16} & $(0,1,1)$ & 4 \\
\hline & & & & & & & & & & & & $(2,1,1)$ & 12 \\
\hline & & & & & & & & & & $(0,2,1,1)$ & 12 & $(1,2,1)$ & 12 \\
\hline & & & & & & & & \multirow[t]{3}{*}{$(01,0,2)$} & \multirow[t]{3}{*}{21} & \multirow[t]{2}{*}{$(1,1,0,2)$} & \multirow[t]{2}{*}{12} & $(1,1,0)$ & 4 \\
\hline & & & & & & & & & & & & $(3,1,0)$ & 8 \\
\hline & & & & & & & & & & $(0,2,0,2)$ & 9 & $(2,2,0)$ & 9 \\
\hline & & & & & & \multirow[t]{2}{*}{$(00,01)$} & \multirow[t]{2}{*}{7} & $(00,1,1)$ & 4 & $(0,0,1,1)$ & 4 & $(1,0,1)$ & 4 \\
\hline & & & & & & & & $(00,0,2)$ & 3 & $(0,0,0,2)$ & 3 & $(2,0,0)$ & 3 \\
\hline & & & & \multirow[t]{3}{*}{$(001,00)$} & \multirow[t]{3}{*}{8} & \multirow[t]{2}{*}{$(01,00)$} & \multirow[t]{2}{*}{7} & \multirow[t]{2}{*}{$(01,0,0)$} & \multirow[t]{2}{*}{7} & $(1,1,0,0)$ & 4 & $(1,1,0)$ & 4 \\
\hline & & & & & & & & & & $(0,2,0,0)$ & 3 & $(0,2,0)$ & 3 \\
\hline & & & & & & $(00,00)$ & 1 & $(00,0,0)$ & 1 & $(0,0,0,0)$ & 1 & $(0,0,0)$ & 1 \\
\hline \multicolumn{2}{|l|}{1 S.I. } & \multicolumn{2}{|l|}{1 S.I. } & \multicolumn{2}{|c|}{2 S.I. } & \multicolumn{2}{|c|}{4 S.I. } & 6 S.I & & 9 S.I. & & $11 \mathrm{~s}$. & \\
\hline
\end{tabular}


24. $D_{7} \supset A_{1} \oplus A_{1} \oplus D_{5} \supset A_{1} \oplus A_{1} \oplus A_{4} \supset A_{1} \oplus A_{1} \oplus A_{3} \supset A_{1} \oplus A_{1} \oplus A_{1} \oplus A_{1} \supset\left(A_{1}\right)_{13} \oplus A_{1} \oplus A_{1}$.

\begin{tabular}{|c|c|c|c|c|c|c|c|c|c|c|c|}
\hline \multicolumn{2}{|l|}{$D_{7}$} & \multicolumn{2}{|c|}{$A_{1} \oplus A_{1} \oplus D_{5}$} & \multicolumn{2}{|c|}{$A_{1} \oplus A_{1} \oplus A_{4}$} & \multicolumn{2}{|c|}{$A_{1} \oplus A_{1} \oplus A_{3}$} & \multicolumn{2}{|l|}{$\left(A_{1}\right)^{4}$} & \multicolumn{2}{|c|}{$\left(A_{1}\right)^{3}$} \\
\hline Peso & $d$ & Peso & $d$ & Peso & $d$ & Peso & $d$ & Peso & $d$ & Peso & $d$ \\
\hline \multirow[t]{15}{*}{$(0000010)$} & \multirow[t]{15}{*}{64} & \multirow[t]{6}{*}{$(0,1,00010)$} & \multirow[t]{6}{*}{32} & \multirow[t]{2}{*}{$(0,1,1000)$} & \multirow[t]{2}{*}{10} & $(0,1,100)$ & 8 & $(0,1,1,1)$ & 8 & $(1,1,1)$ & 8 \\
\hline & & & & & & $(0,1,000)$ & 2 & $(0,1,0,0)$ & 2 & $(0,1,0)$ & 2 \\
\hline & & & & \multirow[t]{3}{*}{$(0,1,0010)$} & \multirow[t]{3}{*}{20} & \multirow[t]{2}{*}{$(0,1,010)$} & \multirow[t]{2}{*}{12} & $(0,1,2,0)$ & 6 & $(2,1,0)$ & 6 \\
\hline & & & & & & & & $(0,1,0,2)$ & 6 & $(0,1,2)$ & 6 \\
\hline & & & & & & $(0,1,001)$ & 8 & $(0,1,1,1)$ & 8 & $(1,1,1)$ & 8 \\
\hline & & & & $(0,1,0000)$ & 2 & $(0,1,000)$ & 2 & $(0,1,0,0)$ & 2 & $(0,1,0)$ & 2 \\
\hline & & \multirow[t]{9}{*}{$(1,0,00001)$} & \multirow[t]{9}{*}{32} & \multirow[t]{3}{*}{$(1,0,0001)$} & \multirow[t]{3}{*}{10} & \multirow[t]{2}{*}{$(1,0,001)$} & \multirow[t]{2}{*}{8} & \multirow[t]{2}{*}{$(1,0,1,1)$} & \multirow[t]{2}{*}{8} & $(0,0,1)$ & 2 \\
\hline & & & & & & & & & & $(2,0,1)$ & 6 \\
\hline & & & & & & $(1,0,000)$ & 2 & $(1,0,0,0)$ & 2 & $(1,0,0)$ & 2 \\
\hline & & & & \multirow[t]{5}{*}{$(1,0,0100)$} & \multirow[t]{5}{*}{20} & \multirow[t]{3}{*}{$(1,0,010)$} & \multirow[t]{3}{*}{12} & \multirow[t]{2}{*}{$(1,0,2,0)$} & \multirow[t]{2}{*}{6} & $(1,0,0)$ & 2 \\
\hline & & & & & & & & & & $(3,0,0)$ & 4 \\
\hline & & & & & & & & $(1,0,0,2)$ & 6 & $(1,0,2)$ & 6 \\
\hline & & & & & & \multirow[t]{2}{*}{$(1,0,100)$} & \multirow[t]{2}{*}{8} & \multirow[t]{2}{*}{$(1,0,1,1)$} & \multirow[t]{2}{*}{8} & $(0,0,1)$ & 2 \\
\hline & & & & & & & & & & $(2,0,1)$ & 6 \\
\hline & & & & $(1,0,0000)$ & 2 & $(1,0,000)$ & 2 & $(1,0,0,0)$ & 2 & $(1,0,0)$ & 2 \\
\hline 1 S.I. & & 2 S.I. & & 6 S.I. & & 10 S.I & & 12 S.I. & & $15 \mathrm{~s}$ & \\
\hline
\end{tabular}


25. $D_{7} \supset A_{1} \oplus A_{1} \oplus D_{5} \supset A_{1} \oplus A_{1} \oplus A_{4} \supset A_{1} \oplus A_{1} \oplus A_{1} \oplus A_{2} \supset A_{1} \oplus A_{1} \oplus A_{1} \oplus \tilde{A}_{1} \supset\left(A_{1}\right)_{13} \oplus A_{1} \oplus A_{1}$.

\begin{tabular}{|c|c|c|c|c|c|c|c|c|c|c|c|}
\hline \multicolumn{2}{|l|}{$D_{7}$} & \multicolumn{2}{|c|}{$\left(A_{1}\right)^{2} \oplus D_{5}$} & \multicolumn{2}{|c|}{$\left(A_{1}\right)^{2} \oplus A_{4}$} & \multicolumn{2}{|c|}{$\left(A_{1}\right)^{3} \oplus A_{2}$} & \multicolumn{2}{|l|}{$\left(A_{1}\right)^{4}$} & \multicolumn{2}{|c|}{$\left(A_{1}\right)^{3}$} \\
\hline Peso & $d$ & Peso & $d$ & Peso & $d$ & Peso & $d$ & Peso & $d$ & Peso & $d$ \\
\hline \multirow[t]{14}{*}{ (0000010) } & \multirow[t]{14}{*}{64} & \multirow[t]{6}{*}{$(0,1,00010)$} & \multirow[t]{6}{*}{32} & \multirow[t]{2}{*}{$(0,1,1000)$} & \multirow[t]{2}{*}{10} & $(0,1,0,10)$ & 6 & $(0,1,0,2)$ & 6 & $(0,1,2)$ & 6 \\
\hline & & & & & & $(0,1,1,00)$ & 4 & $(0,1,1,0)$ & 4 & $(1,1,0)$ & 4 \\
\hline & & & & \multirow[t]{3}{*}{$(0,1,0010)$} & \multirow[t]{3}{*}{20} & $(0,1,1,01)$ & 12 & $(0,1,1,2)$ & 12 & $(1,1,2)$ & 12 \\
\hline & & & & & & $(0,1,0,10)$ & 6 & $(0,1,0,2)$ & 6 & $(0,1,2)$ & 6 \\
\hline & & & & & & $(0,1,0,00)$ & 2 & $(0,1,0,0)$ & 2 & $(0,1,0)$ & 2 \\
\hline & & & & $(0,1,0000)$ & 2 & $(0,1,0,00)$ & 2 & $(0,1,0,0)$ & 2 & $(0,1,0)$ & 2 \\
\hline & & \multirow[t]{8}{*}{$(1,0,00001)$} & \multirow[t]{8}{*}{32} & \multirow[t]{3}{*}{$(1,0,0001)$} & \multirow[t]{3}{*}{10} & $(1,0,0,01)$ & 6 & $(1,0,0,2)$ & 6 & $(1,0,2)$ & 6 \\
\hline & & & & & & \multirow[t]{2}{*}{$(1,0,1,00)$} & \multirow[t]{2}{*}{4} & \multirow[t]{2}{*}{$(1,0,1,0)$} & \multirow[t]{2}{*}{4} & $(0,0,0)$ & 1 \\
\hline & & & & & & & & & & $(2,0,0)$ & 3 \\
\hline & & & & \multirow[t]{4}{*}{$(1,0,0100)$} & \multirow[t]{4}{*}{20} & \multirow[t]{2}{*}{$(1,0,1,10)$} & \multirow[t]{2}{*}{12} & \multirow[t]{2}{*}{$(1,0,1,2)$} & \multirow[t]{2}{*}{12} & $(0,0,2)$ & 3 \\
\hline & & & & & & & & & & $(2,0,0)$ & 3 \\
\hline & & & & & & $(1,0,0,01)$ & 6 & $(1,0,0,2)$ & 6 & $(1,0,2)$ & 6 \\
\hline & & & & & & $(1,0,0,00)$ & 2 & $(1,0,0,0)$ & 2 & $(1,0,0)$ & 2 \\
\hline & & & & $(1,0,0000)$ & 2 & $(1,0,0,00)$ & 2 & $(1,0,0,0)$ & 2 & $(1,0,0)$ & 2 \\
\hline \multicolumn{2}{|l|}{1 S.I. } & \multicolumn{2}{|l|}{2 S.I. } & \multicolumn{2}{|l|}{6 S.I. } & \multicolumn{2}{|l|}{12 S.I. } & \multicolumn{2}{|l|}{12 S.I. } & \multicolumn{2}{|c|}{14 S.I. } \\
\hline
\end{tabular}


26. $D_{7} \supset A_{1} \oplus A_{1} \oplus D_{5} \supset A_{1} \oplus A_{1} \oplus A_{4} \supset A_{1} \oplus A_{1} \oplus A_{1} \oplus A_{2} \supset A_{1} \oplus A_{1} \oplus A_{1} \oplus \tilde{A}_{1} \supset\left(A_{1}\right)_{14} \oplus A_{1} \oplus A_{1}$.

\begin{tabular}{|c|c|c|c|c|c|c|c|c|c|c|c|}
\hline \multicolumn{2}{|l|}{$D_{7}$} & \multicolumn{2}{|c|}{$\left(A_{1}\right)^{2} \oplus D_{5}$} & \multicolumn{2}{|c|}{$\left(A_{1}\right)^{2} \oplus A_{4}$} & \multicolumn{2}{|c|}{$\left(A_{1}\right)^{3} \oplus A_{2}$} & \multicolumn{2}{|c|}{$\left(A_{1}\right)^{4}$} & \multicolumn{2}{|c|}{$\left(A_{1}\right)^{3}$} \\
\hline Peso & $d$ & Peso & $d$ & Peso & $d$ & Peso & $d$ & Peso & $d$ & Peso & $d$ \\
\hline \multirow[t]{15}{*}{ (0000010) } & \multirow[t]{15}{*}{64} & \multirow[t]{6}{*}{$(0,1,00010)$} & \multirow[t]{6}{*}{32} & \multirow[t]{2}{*}{$(0,1,1000)$} & \multirow[t]{2}{*}{10} & $(0,1,0,10)$ & 6 & $(0,1,0,2)$ & 6 & $(2,1,0)$ & 6 \\
\hline & & & & & & $(0,1,1,00)$ & 4 & $(0,1,1,0)$ & 4 & $(0,1,1)$ & 4 \\
\hline & & & & \multirow[t]{3}{*}{$(0,1,0010)$} & \multirow[t]{3}{*}{20} & $(0,1,1,01)$ & 12 & $(0,1,1,2)$ & 12 & $(2,1,1)$ & 12 \\
\hline & & & & & & $(0,1,0,10)$ & 6 & $(0,1,0,2)$ & 6 & $(2,1,0)$ & 6 \\
\hline & & & & & & $(0,1,0,00)$ & 2 & $(0,1,0,0)$ & 2 & $(0,1,0)$ & 2 \\
\hline & & & & $(0,1,0000)$ & 2 & $(0,1,0,00)$ & 2 & $(0,1,0,0)$ & 2 & $(0,1,0)$ & 2 \\
\hline & & \multirow[t]{9}{*}{$(1,0,00001)$} & \multirow[t]{9}{*}{32} & \multirow[t]{3}{*}{$(1,0,0001)$} & \multirow[t]{3}{*}{10} & \multirow[t]{2}{*}{$(1,0,0,01)$} & \multirow[t]{2}{*}{6} & \multirow[t]{2}{*}{$(1,0,0,2)$} & \multirow[t]{2}{*}{6} & $(1,0,0)$ & 2 \\
\hline & & & & & & & & & & $(3,0,0)$ & 4 \\
\hline & & & & & & $(1,0,1,00)$ & 4 & $(1,0,1,0)$ & 4 & $(1,0,1)$ & 1 \\
\hline & & & & \multirow[t]{5}{*}{$(1,0,0100)$} & \multirow[t]{5}{*}{20} & \multirow[t]{2}{*}{$(1,0,1,10)$} & \multirow[t]{2}{*}{12} & \multirow[t]{2}{*}{$(1,0,1,2)$} & \multirow[t]{2}{*}{12} & $(1,0,1)$ & 4 \\
\hline & & & & & & & & & & $(3,0,1)$ & 8 \\
\hline & & & & & & \multirow[t]{2}{*}{$(1,0,0,01)$} & \multirow[t]{2}{*}{6} & \multirow[t]{2}{*}{$(1,0,0,2)$} & \multirow[t]{2}{*}{6} & $(1,0,0)$ & 2 \\
\hline & & & & & & & & & & $(3,0,0)$ & 4 \\
\hline & & & & & & $(1,0,0,00)$ & 2 & $(1,0,0,0)$ & 2 & $(1,0,0)$ & 2 \\
\hline & & & & $(1,0,0000)$ & 2 & $(1,0,0,00)$ & 2 & $(1,0,0,0)$ & 2 & $(1,0,0)$ & 2 \\
\hline \multicolumn{2}{|l|}{1 S.I. } & \multicolumn{2}{|l|}{2 S.I. } & 6 S.I. & & 12 S.I. & & 12 S.I & & 15 & \\
\hline
\end{tabular}


27. $D_{7} \supset A_{1} \oplus A_{1} \oplus D_{5} \supset A_{1} \oplus A_{1} \oplus A_{4} \supset A_{1} \oplus A_{1} \oplus A_{1} \oplus A_{2} \supset A_{1} \oplus A_{1} \oplus A_{1} \oplus \tilde{A}_{1} \supset\left(A_{1}\right)_{34} \oplus A_{1} \oplus A_{1}$.

\begin{tabular}{|c|c|c|c|c|c|c|c|c|c|c|c|}
\hline \multicolumn{2}{|l|}{$D_{7}$} & \multicolumn{2}{|c|}{$\left(A_{1}\right)^{2} \oplus D_{5}$} & \multicolumn{2}{|c|}{$\left(A_{1}\right)^{2} \oplus A_{4}$} & \multicolumn{2}{|c|}{$\left(A_{1}\right)^{3} \oplus A_{2}$} & \multicolumn{2}{|l|}{$\left(A_{1}\right)^{4}$} & \multicolumn{2}{|c|}{$\left(A_{1}\right)^{3}$} \\
\hline Peso & $d$ & Peso & $d$ & Peso & $d$ & Peso & $d$ & Peso & $d$ & Peso & $d$ \\
\hline \multirow[t]{14}{*}{$(0000010)$} & \multirow[t]{14}{*}{64} & \multirow[t]{7}{*}{$(0,1,00010)$} & \multirow[t]{7}{*}{32} & \multirow[t]{2}{*}{$(0,1,1000)$} & \multirow[t]{2}{*}{10} & $(0,1,0,10)$ & 6 & $(0,1,0,2)$ & 6 & $(2,0,1)$ & 6 \\
\hline & & & & & & $(0,1,1,00)$ & 4 & $(0,1,1,0)$ & 4 & $(1,0,1)$ & 4 \\
\hline & & & & \multirow[t]{4}{*}{$(0,1,0010)$} & \multirow[t]{4}{*}{20} & \multirow[t]{2}{*}{$(0,1,1,01)$} & \multirow[t]{2}{*}{12} & \multirow[t]{2}{*}{$(0,1,1,2)$} & \multirow[t]{2}{*}{12} & $(1,0,1)$ & 4 \\
\hline & & & & & & & & & & $(3,0,1)$ & 8 \\
\hline & & & & & & $(0,1,0,10)$ & 6 & $(0,1,0,2)$ & 6 & $(2,0,1)$ & 6 \\
\hline & & & & & & $(0,1,0,00)$ & 2 & $(0,1,0,0)$ & 2 & $(0,0,1)$ & 2 \\
\hline & & & & $(0,1,0000)$ & 2 & $(0,1,0,00)$ & 2 & $(0,1,0,0)$ & 2 & $(0,0,1)$ & 2 \\
\hline & & \multirow[t]{7}{*}{$(1,0,00001)$} & \multirow[t]{7}{*}{32} & \multirow[t]{2}{*}{$(1,0,0001)$} & \multirow[t]{2}{*}{10} & $(1,0,0,01)$ & 6 & $(1,0,0,2)$ & 6 & $(2,1,0)$ & 6 \\
\hline & & & & & & $(1,0,1,00)$ & 4 & $(1,0,1,0)$ & 4 & $(1,1,0)$ & 4 \\
\hline & & & & \multirow[t]{4}{*}{$(1,0,0100)$} & \multirow[t]{4}{*}{20} & \multirow[t]{2}{*}{$(1,0,1,10)$} & \multirow[t]{2}{*}{12} & \multirow[t]{2}{*}{$(1,0,1,2)$} & \multirow[t]{2}{*}{12} & $(1,1,0)$ & 4 \\
\hline & & & & & & & & & & $(3,1,0)$ & 8 \\
\hline & & & & & & $(1,0,0,01)$ & 6 & $(1,0,0,2)$ & 6 & $(2,1,0)$ & 6 \\
\hline & & & & & & $(1,0,0,00)$ & 2 & $(1,0,0,0)$ & 2 & $(0,1,0)$ & 2 \\
\hline & & & & $(1,0,0000)$ & 2 & $(1,0,0,00)$ & 2 & $(1,0,0,0)$ & 2 & $(0,1,0)$ & 2 \\
\hline \multicolumn{2}{|l|}{1 S.I. } & \multicolumn{2}{|l|}{2 S.I. } & \multicolumn{2}{|l|}{6 S.I. } & \multicolumn{2}{|l|}{12 S.I. } & \multicolumn{2}{|l|}{12 S.I. } & \multicolumn{2}{|c|}{14 S.I. } \\
\hline
\end{tabular}


28. $D_{7} \supset A_{3} \oplus D_{4} \supset A_{3} \oplus A_{1} \oplus C_{2} \supset C_{2} \oplus A_{1} \oplus C_{2} \supset A_{1} \oplus A_{1} \oplus A_{1} \oplus C_{2} \supset$

$\supset A_{1} \oplus A_{1} \oplus A_{1} \oplus A_{1} \oplus A_{1} \supset\left(A_{1}\right)_{14} \oplus A_{1} \oplus A_{1} \oplus A_{1} \supset\left(A_{1}\right)_{24} \oplus A_{1} \oplus A_{1}$.

\begin{tabular}{|c|c|c|c|c|c|c|c|c|c|c|c|c|c|c|c|}
\hline \multicolumn{2}{|l|}{$D_{7}$} & \multicolumn{2}{|l|}{$A_{3} \oplus D_{4}$} & \multicolumn{2}{|c|}{$A_{3} \oplus A_{1} \oplus C_{2}$} & \multicolumn{2}{|c|}{$C_{2} \oplus A_{1} \oplus C_{2}$} & \multicolumn{2}{|c|}{$\left(A_{1}\right)^{3} \oplus C_{2}$} & \multicolumn{2}{|l|}{$\left(A_{1}\right)^{5}$} & \multicolumn{2}{|l|}{$\left(A_{1}\right)^{4}$} & \multicolumn{2}{|c|}{$\left(A_{1}\right)^{3}$} \\
\hline Peso & $d$ & Peso & $d$ & Peso & $d$ & Peso & $d$ & Peso & $d$ & Peso & $d$ & Peso & $d$ & Peso & $d$ \\
\hline \multirow[t]{14}{*}{$(0000010)$} & \multirow[t]{14}{*}{64} & \multirow[t]{8}{*}{$(001,0010)$} & \multirow[t]{8}{*}{32} & \multirow[t]{6}{*}{$(001,0,01)$} & \multirow[t]{6}{*}{20} & \multirow[t]{6}{*}{$(10,0,01)$} & \multirow[t]{6}{*}{20} & \multirow[t]{3}{*}{$(1,0,0,01)$} & \multirow[t]{3}{*}{10} & \multirow[t]{2}{*}{$(1,0,0,1,1)$} & \multirow[t]{2}{*}{8} & $(0,0,0,1)$ & 2 & $(1,0,0)$ & 2 \\
\hline & & & & & & & & & & & & $(2,0,0,1)$ & 6 & $(1,2,0)$ & 6 \\
\hline & & & & & & & & & & $(1,0,0,0,0)$ & 2 & $(1,0,0,0)$ & 2 & $(0,1,0)$ & 2 \\
\hline & & & & & & & & \multirow[t]{3}{*}{$(0,1,0,01)$} & \multirow[t]{3}{*}{10} & \multirow{2}{*}{$(0,1,0,1,1)$} & \multirow[t]{2}{*}{8} & \multirow{2}{*}{$(1,1,0,1)$} & \multirow[t]{2}{*}{8} & $(0,1,0)$ & 2 \\
\hline & & & & & & & & & & & & & & $(2,1,0)$ & 6 \\
\hline & & & & & & & & & & $(0,1,0,0,0)$ & 2 & $(0,1,0,0)$ & 2 & $(1,0,0)$ & 2 \\
\hline & & & & \multirow[t]{2}{*}{$(001,2,00)$} & \multirow[t]{2}{*}{12} & \multirow[t]{2}{*}{$(10,2,00)$} & \multirow[t]{2}{*}{12} & $(1,0,2,00)$ & 6 & $(1,0,2,0,0)$ & 6 & $(1,0,2,0)$ & 6 & $(0,1,2)$ & 6 \\
\hline & & & & & & & & $(0,1,2,00)$ & 6 & $(0,1,2,0,0)$ & 6 & $(0,1,2,0)$ & 6 & $(1,0,2)$ & 6 \\
\hline & & \multirow[t]{6}{*}{$(100,0001)$} & \multirow[t]{6}{*}{32} & \multirow[t]{6}{*}{$(100,1,10)$} & 32 & $(10,1,10)$ & 32 & $(1,0,1,10)$ & 16 & $(1,0,1,1,0)$ & 8 & $(0,0,1,0)$ & 2 & $(0,0,1)$ & 2 \\
\hline & & & & & & & & & & & & $(2,0,1,0)$ & 6 & $(0,2,1)$ & 6 \\
\hline & & & & & & & & & & $(1,0,1,0,1)$ & 8 & $(1,0,1,1)$ & 8 & $(1,1,1)$ & 8 \\
\hline & & & & & & & & $(0,1,1,10)$ & 16 & $(0,1,1,1,0)$ & 8 & $(1,1,1,0)$ & 8 & $(1,1,1)$ & 8 \\
\hline & & & & & & & & & & $(0,1,1,0,1)$ & 8 & $(0,1,1,1)$ & 8 & $(0,0,1)$ & 2 \\
\hline & & & & & & & & & & & & & & $(2,0,1)$ & 6 \\
\hline 1 S.I. & & 2 S.I. & & 3 S.I. & & 3 S.I. & & 6 S.I. & & 10 S.I. & & 12 S.I. & & 14 S.I. & \\
\hline
\end{tabular}


29. $D_{7} \supset A_{3} \oplus D_{4} \supset A_{3} \oplus A_{1} \oplus C_{2} \supset A_{1} \oplus A_{1} \oplus A_{1} \oplus C_{2} \supset A_{1} \oplus A_{1} \oplus A_{1} \oplus A_{1} \oplus A_{1} \supset\left(A_{1}\right)_{13} \oplus A_{1} \oplus A_{1} \oplus A_{1} \supset\left(A_{1}\right)_{24} \oplus A_{1} \oplus A_{1}$.

\begin{tabular}{|c|c|c|c|c|c|c|c|c|c|c|c|c|c|}
\hline \multicolumn{2}{|l|}{$D_{7}$} & \multicolumn{2}{|l|}{$A_{3} \oplus D_{4}$} & \multicolumn{2}{|c|}{$A_{3} \oplus A_{1} \oplus C_{2}$} & \multicolumn{2}{|c|}{$\left(A_{1}\right)^{3} \oplus C_{2}$} & \multicolumn{2}{|l|}{$\left(A_{1}\right)^{5}$} & \multicolumn{2}{|l|}{$\left(A_{1}\right)^{4}$} & \multicolumn{2}{|c|}{$\left(A_{1}\right)^{3}$} \\
\hline Peso & $d$ & Peso & $d$ & Peso & $d$ & Peso & $d$ & Peso & $d$ & Peso & $d$ & Peso & $d$ \\
\hline \multirow[t]{9}{*}{$(0000010)$} & \multirow[t]{9}{*}{64} & \multirow[t]{5}{*}{$(001,0010)$} & \multirow[t]{5}{*}{32} & \multirow[t]{3}{*}{$(001,0,01)$} & \multirow[t]{3}{*}{20} & \multirow[t]{3}{*}{$(1,1,0,01)$} & \multirow[t]{3}{*}{20} & \multirow[t]{2}{*}{$(1,1,0,1,1)$} & \multirow[t]{2}{*}{16} & \multirow[t]{2}{*}{$(1,1,1,1)$} & \multirow[t]{2}{*}{16} & $(0,1,1)$ & 4 \\
\hline & & & & & & & & & & & & $(2,1,1)$ & 12 \\
\hline & & & & & & & & $(1,1,0,0,0)$ & 4 & $(1,1,0,0)$ & 4 & $(0,1,1)$ & 4 \\
\hline & & & & \multirow[t]{2}{*}{$(001,2,00)$} & \multirow[t]{2}{*}{12} & \multirow[t]{2}{*}{$(1,1,2,00)$} & \multirow[t]{2}{*}{12} & \multirow[t]{2}{*}{$(1,1,2,0,0)$} & \multirow[t]{2}{*}{12} & $(1,1,0,0)$ & 4 & $(0,1,1)$ & 4 \\
\hline & & & & & & & & & & $(3,1,0,0)$ & 8 & $(0,3,1)$ & 8 \\
\hline & & \multirow[t]{4}{*}{$(100,0001)$} & \multirow[t]{4}{*}{32} & \multirow[t]{4}{*}{$(100,1,10)$} & \multirow[t]{4}{*}{32} & \multirow[t]{4}{*}{$(1,1,1,10)$} & \multirow[t]{4}{*}{32} & \multirow[t]{2}{*}{$(1,1,1,1,0)$} & \multirow[t]{2}{*}{16} & $(0,1,1,0)$ & 4 & $(1,0,1)$ & 4 \\
\hline & & & & & & & & & & $(2,1,1,0)$ & 12 & $(1,2,1)$ & 12 \\
\hline & & & & & & & & \multirow[t]{2}{*}{$(1,1,1,0,1)$} & \multirow[t]{2}{*}{16} & $(0,1,0,1)$ & 4 & $(1,0,1)$ & 4 \\
\hline & & & & & & & & & & $(2,1,0,1)$ & 12 & $(1,2,1)$ & 12 \\
\hline \multicolumn{2}{|l|}{1 S.I. } & 2 S.I. & & 3 S.I. & & 3 S.I. & & 5 S.I. & & 8 S.I. & & 9 S.I. & \\
\hline
\end{tabular}

30. $D_{7} \supset A_{3} \oplus D_{4} \supset A_{3} \oplus A_{1} \oplus C_{2} \supset A_{2} \oplus A_{1} \oplus C_{2} \supset A_{1} \oplus A_{1} \oplus C_{2} \supset A_{1} \oplus A_{1} \oplus A_{1} \supset\left(A_{1}\right)_{12} \oplus A_{1}$.

\begin{tabular}{|c|c|c|c|c|c|c|c|c|c|c|c|c|c|}
\hline \multicolumn{2}{|l|}{$D_{7}$} & \multicolumn{2}{|c|}{$A_{3} \oplus D_{4}$} & \multicolumn{2}{|c|}{$A_{3} \oplus A_{1} \oplus C_{2}$} & \multicolumn{2}{|c|}{$A_{2} \oplus A_{1} \oplus C_{2}$} & \multicolumn{2}{|c|}{$\left(A_{1}\right)^{2} \oplus C_{2}$} & \multicolumn{2}{|c|}{$\left(A_{1}\right)^{3}$} & \multicolumn{2}{|c|}{$\left(A_{1}\right)^{2}$} \\
\hline Peso & $d$ & Peso & $d$ & Peso & $d$ & Peso & $d$ & Peso & $d$ & Peso & $d$ & Peso & $d$ \\
\hline \multirow[t]{11}{*}{ (0000010) } & \multirow[t]{11}{*}{64} & \multirow[t]{7}{*}{$(001,0010)$} & \multirow[t]{7}{*}{32} & \multirow[t]{4}{*}{$(001,0,01)$} & \multirow[t]{4}{*}{20} & \multirow[t]{3}{*}{$(01,0,01)$} & \multirow[t]{3}{*}{15} & \multirow[t]{2}{*}{$(1,0,01)$} & \multirow[t]{2}{*}{10} & \multirow[t]{2}{*}{$(1,0,4)$} & \multirow[t]{2}{*}{10} & $(3,0)$ & 4 \\
\hline & & & & & & & & & & & & $(5,0)$ & 6 \\
\hline & & & & & & & & $(0,0,01)$ & 5 & $(0,0,4)$ & 5 & $(4,0)$ & 5 \\
\hline & & & & & & $(00,0,01)$ & 5 & $(0,0,01)$ & 5 & $(0,0,4)$ & 5 & $(4,0)$ & 5 \\
\hline & & & & \multirow[t]{3}{*}{$(001,2,00)$} & \multirow[t]{3}{*}{12} & \multirow[t]{2}{*}{$(01,2,00)$} & \multirow[t]{2}{*}{9} & $(1,2,00)$ & 6 & $(1,2,0)$ & 6 & $(1,2)$ & 6 \\
\hline & & & & & & & & $(0,2,00)$ & 3 & $(0,2,0)$ & 3 & $(0,2)$ & 3 \\
\hline & & & & & & $(00,2,00)$ & 3 & $(0,2,00)$ & 3 & $(0,2,0)$ & 3 & $(0,2)$ & 3 \\
\hline & & \multirow[t]{4}{*}{$(100,0001)$} & \multirow[t]{4}{*}{32} & \multirow[t]{4}{*}{$(100,1,10)$} & \multirow[t]{4}{*}{32} & \multirow[t]{3}{*}{$(10,1,10)$} & \multirow[t]{3}{*}{24} & \multirow[t]{2}{*}{$(1,1,10)$} & \multirow[t]{2}{*}{16} & \multirow[t]{2}{*}{$(1,1,3)$} & \multirow[t]{2}{*}{16} & $(2,1)$ & 6 \\
\hline & & & & & & & & & & & & $(4,1)$ & 10 \\
\hline & & & & & & & & $(0,1,10)$ & 8 & $(0,1,3)$ & 8 & $(3,1)$ & 8 \\
\hline & & & & & & $(00,1,10)$ & 8 & $(0,1,10)$ & 8 & $(0,1,3)$ & 8 & $(3,1)$ & 8 \\
\hline \multicolumn{2}{|l|}{1 S.I. } & \multicolumn{2}{|l|}{2 S.I. } & 3 S.I. & & 6 S.I. & & 9 S.I. & & 9 S.I & & 11 & \\
\hline
\end{tabular}


31. $D_{7} \supset A_{3} \oplus D_{4} \supset A_{3} \oplus A_{1} \oplus C_{2} \supset C_{2} \oplus A_{1} \oplus C_{2} \supset A_{1} \oplus A_{1} \oplus C_{2} \supset A_{1} \oplus A_{1} \oplus A_{1} \supset\left(A_{1}\right)_{13} \oplus A_{1}$.

\begin{tabular}{|c|c|c|c|c|c|c|c|c|c|c|c|c|c|}
\hline \multicolumn{2}{|l|}{$D_{7}$} & \multicolumn{2}{|c|}{$A_{3} \oplus D_{4}$} & \multicolumn{2}{|c|}{$A_{3} \oplus A_{1} \oplus C_{2}$} & \multicolumn{2}{|c|}{$C_{2} \oplus A_{1} \oplus C_{2}$} & \multicolumn{2}{|c|}{$\left(A_{1}\right)^{2} \oplus C_{2}$} & \multicolumn{2}{|c|}{$\left(A_{1}\right)^{3}$} & \multicolumn{2}{|c|}{$\left(A_{1}\right)^{2}$} \\
\hline Peso & $d$ & Peso & $d$ & Peso & $d$ & Peso & $d$ & Peso & $d$ & Peso & $d$ & Peso & $d$ \\
\hline \multirow[t]{9}{*}{ (0000010) } & \multirow[t]{9}{*}{64} & \multirow[t]{5}{*}{$(001,0010)$} & \multirow[t]{5}{*}{32} & \multirow[t]{4}{*}{$(001,0,01)$} & \multirow[t]{4}{*}{20} & \multirow[t]{4}{*}{$(01,0,01)$} & \multirow[t]{4}{*}{20} & \multirow[t]{4}{*}{$(3,0,01)$} & \multirow[t]{4}{*}{20} & \multirow[t]{4}{*}{$(3,0,4)$} & \multirow[t]{4}{*}{20} & $(1,0)$ & 2 \\
\hline & & & & & & & & & & & & $(3,0)$ & 4 \\
\hline & & & & & & & & & & & & $(5,0)$ & 6 \\
\hline & & & & & & & & & & & & $(7,0)$ & 8 \\
\hline & & & & $(001,2,00)$ & 12 & $(01,2,00)$ & 12 & $(3,2,00)$ & 12 & $(3,2,0)$ & 12 & $(3,2)$ & 12 \\
\hline & & \multirow[t]{4}{*}{$(100,0001)$} & \multirow[t]{4}{*}{32} & \multirow[t]{4}{*}{$(100,1,10)$} & \multirow[t]{4}{*}{32} & \multirow[t]{4}{*}{$(10,1,10)$} & \multirow[t]{4}{*}{32} & \multirow[t]{4}{*}{$(3,1,10)$} & \multirow[t]{4}{*}{32} & \multirow[t]{4}{*}{$(3,1,3)$} & \multirow[t]{4}{*}{32} & $(0,1)$ & 2 \\
\hline & & & & & & & & & & & & $(2,1)$ & 6 \\
\hline & & & & & & & & & & & & $(4,1)$ & 10 \\
\hline & & & & & & & & & & & & $(6,1)$ & 14 \\
\hline \multicolumn{2}{|l|}{1 S.I. } & \multicolumn{2}{|l|}{2 S.I. } & \multicolumn{2}{|l|}{3 S.I. } & \multicolumn{2}{|l|}{3 S.I. } & \multicolumn{2}{|c|}{3 S.I. } & \multicolumn{2}{|c|}{3 S.I. } & \multicolumn{2}{|c|}{9 S.I. } \\
\hline
\end{tabular}

32. $D_{7} \supset B_{3} \oplus B_{3} \supset B_{3} \oplus G_{2} \supset G_{2} \oplus G_{2} \supset G_{2} \oplus A_{1} \supset A_{1} \oplus A_{1} \oplus A_{1} \supset\left(A_{1}\right)_{13} \oplus A_{1}$.

\begin{tabular}{|c|c|c|c|c|c|c|c|c|c|c|c|c|c|}
\hline \multicolumn{2}{|l|}{$D_{7}$} & \multicolumn{2}{|c|}{$B_{3} \oplus B_{3}$} & \multicolumn{2}{|c|}{$B_{3} \oplus G_{2}$} & \multicolumn{2}{|c|}{$G_{2} \oplus G_{2}$} & \multicolumn{2}{|c|}{$G_{2} \oplus A_{1}$} & \multicolumn{2}{|c|}{$\left(A_{1}\right)^{3}$} & \multicolumn{2}{|c|}{$\left(A_{1}\right)^{2}$} \\
\hline Peso & $d$ & Peso & $d$ & Peso & $d$ & Peso & $d$ & Peso & $d$ & Peso & $d$ & Peso & $d$ \\
\hline \multirow[t]{7}{*}{$(0000010)$} & \multirow[t]{7}{*}{64} & \multirow[t]{7}{*}{$(001,001)$} & \multirow[t]{7}{*}{64} & \multirow[t]{4}{*}{$(001,01)$} & \multirow[t]{4}{*}{49} & \multirow[t]{3}{*}{$(01,01)$} & \multirow[t]{3}{*}{6} & \multirow[t]{3}{*}{$(01,6)$} & \multirow[t]{3}{*}{49} & \multirow[t]{2}{*}{$(1,1,6)$} & \multirow[t]{2}{*}{28} & $(5,1)$ & 12 \\
\hline & & & & & & & & & & & & $(7,1)$ & 16 \\
\hline & & & & & & & & & & $(0,2,6)$ & 21 & $(6,2)$ & 21 \\
\hline & & & & & & $(00,01)$ & 7 & $(00,6)$ & 7 & $(0,0,6)$ & 7 & $(6,0)$ & 7 \\
\hline & & & & \multirow[t]{3}{*}{$(001,00)$} & \multirow[t]{3}{*}{8} & \multirow[t]{2}{*}{$(01,00)$} & \multirow[t]{2}{*}{7} & \multirow[t]{2}{*}{$(01,0)$} & \multirow[t]{2}{*}{7} & $(1,1,0)$ & 4 & $(1,1)$ & 4 \\
\hline & & & & & & & & & & $(0,2,0)$ & 3 & $(0,2)$ & 3 \\
\hline & & & & & & $(00,00)$ & 1 & $(00,0)$ & 1 & $(0,0,0)$ & 1 & $(0,0)$ & 1 \\
\hline \multicolumn{2}{|l|}{1 S.I. } & \multicolumn{2}{|l|}{1 S.I. } & \multicolumn{2}{|l|}{2 S.I. } & \multicolumn{2}{|l|}{4 S.I. } & \multicolumn{2}{|c|}{4 S.I. } & \multicolumn{2}{|c|}{6 S.I. } & \multicolumn{2}{|c|}{7 S.I. } \\
\hline
\end{tabular}


33. $D_{7} \supset A_{3} \oplus D_{4} \supset A_{3} \oplus A_{1} \oplus C_{2} \supset C_{2} \oplus A_{1} \oplus C_{2} \supset A_{1} \oplus A_{1} \oplus A_{1} \oplus C_{2} \supset A_{1} \oplus A_{1} \oplus A_{1} \oplus A_{1} \supset\left(A_{1}\right)_{13} \oplus A_{1} \oplus A_{1} \supset$ $\supset\left(A_{1}\right)_{23} \oplus A_{1}$.

\begin{tabular}{|c|c|c|c|c|c|c|c|c|c|c|c|c|c|c|c|}
\hline \multicolumn{2}{|l|}{$D_{7}$} & \multicolumn{2}{|l|}{$A_{3} \oplus D_{4}$} & \multicolumn{2}{|c|}{$A_{3} \oplus A_{1} \oplus C_{2}$} & \multicolumn{2}{|c|}{$C_{2} \oplus A_{1} \oplus C_{2}$} & \multicolumn{2}{|c|}{$\left(A_{1}\right)^{3} \oplus C_{2}$} & \multicolumn{2}{|l|}{$\left(A_{1}\right)^{4}$} & \multicolumn{2}{|c|}{$\left(A_{1}\right)^{3}$} & \multicolumn{2}{|c|}{$\left(A_{1}\right)^{2}$} \\
\hline Peso & $d$ & Peso & $d$ & Peso & $d$ & Peso & $d$ & Peso & $d$ & Peso & $d$ & Peso & $d$ & Peso & $d$ \\
\hline \multirow[t]{10}{*}{$(0000010)$} & \multirow[t]{10}{*}{64} & \multirow[t]{6}{*}{$(001,0010)$} & \multirow[t]{6}{*}{32} & \multirow[t]{3}{*}{$(001,0,01)$} & \multirow[t]{3}{*}{20} & \multirow[t]{3}{*}{$(10,0,01)$} & \multirow[t]{3}{*}{20} & \multirow{2}{*}{$(1,0,0,01)$} & \multirow[t]{2}{*}{10} & \multirow[t]{2}{*}{$(1,0,0,4)$} & \multirow[t]{2}{*}{10} & $(3,0,0)$ & 4 & $(0,3)$ & 4 \\
\hline & & & & & & & & & & & & $(5,0,0)$ & 6 & $(0,5)$ & 6 \\
\hline & & & & & & & & $(0,1,0,01)$ & 10 & $(0,1,0,4)$ & 10 & $(4,1,0)$ & 10 & $(1,4)$ & 10 \\
\hline & & & & \multirow[t]{3}{*}{$(001,2,00)$} & \multirow[t]{3}{*}{12} & \multirow[t]{3}{*}{$(10,2,00)$} & \multirow[t]{3}{*}{12} & $(1,0,2,00)$ & 6 & $(1,0,2,0)$ & 6 & $(1,0,2)$ & 6 & $(2,1)$ & 6 \\
\hline & & & & & & & & \multirow[t]{2}{*}{$(0,1,2,00)$} & \multirow[t]{2}{*}{6} & \multirow[t]{2}{*}{$(0,1,2,0)$} & \multirow[t]{2}{*}{6} & \multirow[t]{2}{*}{$(0,1,2)$} & \multirow[t]{2}{*}{6} & $(1,0)$ & 2 \\
\hline & & & & & & & & & & & & & & $(3,0)$ & 4 \\
\hline & & \multirow[t]{4}{*}{$(100,0001)$} & \multirow[t]{4}{*}{32} & \multirow[t]{4}{*}{$(100,1,10)$} & \multirow[t]{4}{*}{32} & \multirow[t]{4}{*}{$(10,1,10)$} & 32 & $(1,0,1,10)$ & 16 & $(1,0,1,3)$ & 16 & $(2,0,1)$ & 6 & $(1,2)$ & 6 \\
\hline & & & & & & & & & & & & $(4,0,1)$ & 10 & $(1,4)$ & 10 \\
\hline & & & & & & & & $(0,1,1,10)$ & 16 & $(0,1,1,3)$ & 16 & $(3,1,1)$ & 16 & $(0,3)$ & 4 \\
\hline & & & & & & & & & & & & & & $(2,3)$ & 12 \\
\hline 1 S.I. & & 2 S.I. & & 3 S.I. & & 3 S.I. & & 6 S.I. & & 6 S.I. & & $8 \mathrm{~s}$. & & 10 & \\
\hline
\end{tabular}

34. $D_{7} \supset A_{3} \oplus D_{4} \supset A_{3} \oplus A_{1} \oplus C_{2} \supset C_{2} \oplus A_{1} \oplus C_{2} \supset A_{1} \oplus A_{1} \oplus C_{2} \supset A_{1} \oplus A_{1} \oplus A_{1} \oplus A_{1} \supset\left(A_{1}\right)_{12} \oplus A_{1} \oplus A_{1} \supset\left(A_{1}\right)_{23} \oplus A_{1}$.

\begin{tabular}{|c|c|c|c|c|c|c|c|c|c|c|c|c|c|c|c|}
\hline \multicolumn{2}{|l|}{$D_{7}$} & \multicolumn{2}{|l|}{$A_{3} \oplus D_{4}$} & \multicolumn{2}{|c|}{$A_{3} \oplus A_{1} \oplus C_{2}$} & \multicolumn{2}{|c|}{$C_{2} \oplus A_{1} \oplus C_{2}$} & \multicolumn{2}{|c|}{$\left(A_{1}\right)^{2} \oplus C_{2}$} & \multicolumn{2}{|l|}{$\left(A_{1}\right)^{4}$} & \multicolumn{2}{|c|}{$\left(A_{1}\right)^{3}$} & \multicolumn{2}{|c|}{$\left(A_{1}\right)^{2}$} \\
\hline Peso & $d$ & Peso & $d$ & Peso & $d$ & Peso & $d$ & Peso & $d$ & Peso & $d$ & Peso & $d$ & Peso & $d$ \\
\hline \multirow[t]{10}{*}{$(0000010)$} & \multirow[t]{10}{*}{64} & \multirow[t]{6}{*}{$(001,0010)$} & \multirow[t]{6}{*}{32} & \multirow[t]{3}{*}{$(001,0,01)$} & \multirow[t]{3}{*}{20} & \multirow[t]{3}{*}{$(10,0,01)$} & \multirow[t]{3}{*}{20} & \multirow[t]{3}{*}{$(3,0,01)$} & \multirow[t]{3}{*}{20} & \multirow[t]{2}{*}{$(3,0,1,1)$} & \multirow[t]{2}{*}{16} & $(0,3,0)$ & 4 & $(3,0)$ & 4 \\
\hline & & & & & & & & & & & & $(2,3,0)$ & 12 & $(3,2)$ & 12 \\
\hline & & & & & & & & & & $(3,0,0,0)$ & 4 & $(0,3,0)$ & 4 & $(3,0)$ & 4 \\
\hline & & & & \multirow[t]{3}{*}{$(001,2,00)$} & \multirow[t]{3}{*}{12} & \multirow[t]{3}{*}{$(10,2,00)$} & \multirow[t]{3}{*}{12} & \multirow[t]{3}{*}{$(3,2,00)$} & \multirow[t]{3}{*}{12} & \multirow[t]{3}{*}{$(3,2,0,0)$} & \multirow[t]{3}{*}{12} & \multirow[t]{3}{*}{$(0,3,2)$} & \multirow[t]{3}{*}{12} & $(1,0)$ & 2 \\
\hline & & & & & & & & & & & & & & $(3,0)$ & 4 \\
\hline & & & & & & & & & & & & & & $(5,0)$ & 6 \\
\hline & & \multirow[t]{4}{*}{$(100,0001)$} & \multirow[t]{4}{*}{32} & \multirow[t]{4}{*}{$(100,1,10)$} & \multirow[t]{4}{*}{32} & \multirow[t]{4}{*}{$(10,1,10)$} & 32 & $(3,1,10)$ & 16 & $(3,1,1,0)$ & 16 & $(1,3,1)$ & 16 & $(2,1)$ & 6 \\
\hline & & & & & & & & & & & & & & $(4,1)$ & 10 \\
\hline & & & & & & & & & & $(3,1,0,1)$ & 16 & $(1,3,1)$ & 16 & $(2,1)$ & 6 \\
\hline & & & & & & & & & & & & & & $(4,1)$ & 10 \\
\hline 1 S.I. & & 2 S.I. & & 3 S.I. & & 3 S.I. & & 3 S.I. & & 5 S.I. & & 6 S.I. & & $10 \mathrm{~s}$. & \\
\hline
\end{tabular}


35. $D_{7} \supset A_{3} \oplus D_{4} \supset A_{3} \oplus A_{1} \oplus C_{2} \supset A_{1} \oplus A_{1} \oplus A_{1} \oplus C_{2} \supset A_{1} \oplus A_{1} \oplus A_{1} \oplus A_{1} \supset\left(A_{1}\right)_{13} \oplus A_{1} \oplus A_{1} \supset\left(A_{1}\right)_{23} \oplus A_{1}$.

\begin{tabular}{|c|c|c|c|c|c|c|c|c|c|c|c|c|c|}
\hline \multicolumn{2}{|l|}{$D_{7}$} & \multicolumn{2}{|c|}{$A_{3} \oplus D_{4}$} & \multicolumn{2}{|c|}{$A_{3} \oplus A_{1} \oplus C_{2}$} & \multicolumn{2}{|c|}{$\left(A_{1}\right)^{3} \oplus C_{2}$} & \multicolumn{2}{|c|}{$\left(A_{1}\right)^{4}$} & \multicolumn{2}{|c|}{$\left(A_{1}\right)^{3}$} & \multicolumn{2}{|c|}{$\left(A_{1}\right)^{2}$} \\
\hline Peso & $d$ & Peso & $d$ & Peso & $d$ & Peso & $d$ & Peso & $d$ & Peso & $d$ & Peso & $d$ \\
\hline \multirow[t]{8}{*}{ (0000010) } & \multirow[t]{8}{*}{64} & \multirow[t]{4}{*}{$(001,0010)$} & \multirow[t]{4}{*}{32} & \multirow[t]{2}{*}{$(001,0,01)$} & \multirow[t]{2}{*}{20} & \multirow[t]{2}{*}{$(1,1,0,01)$} & \multirow[t]{2}{*}{20} & \multirow[t]{2}{*}{$(1,1,0,4)$} & \multirow[t]{2}{*}{20} & \multirow[t]{2}{*}{$(1,1,4)$} & \multirow[t]{2}{*}{20} & $(3,1)$ & 8 \\
\hline & & & & & & & & & & & & $(5,1)$ & 12 \\
\hline & & & & \multirow[t]{2}{*}{$(001,2,00)$} & \multirow[t]{2}{*}{12} & \multirow[t]{2}{*}{$(1,1,2,00)$} & \multirow[t]{2}{*}{12} & \multirow[t]{2}{*}{$(1,1,2,0)$} & \multirow[t]{2}{*}{12} & $(1,1,0)$ & 4 & $(1,1)$ & 4 \\
\hline & & & & & & & & & & $(3,1,0)$ & 8 & $(1,3)$ & 8 \\
\hline & & \multirow[t]{4}{*}{$(100,0001)$} & \multirow[t]{4}{*}{32} & \multirow[t]{4}{*}{$(100,1,10)$} & \multirow[t]{4}{*}{32} & \multirow[t]{4}{*}{$(1,1,1,10)$} & \multirow[t]{4}{*}{32} & \multirow[t]{4}{*}{$(1,1,1,3)$} & \multirow[t]{4}{*}{32} & \multirow[t]{2}{*}{$(0,1,3)$} & \multirow[t]{2}{*}{8} & $(2,0)$ & 3 \\
\hline & & & & & & & & & & & & $(4,0)$ & 5 \\
\hline & & & & & & & & & & \multirow[t]{2}{*}{$(2,1,3)$} & \multirow[t]{2}{*}{24} & $(2,2)$ & 9 \\
\hline & & & & & & & & & & & & $(4,2)$ & 15 \\
\hline 1 S.I. & & 2 S.I. & & 3 S.I. & & 3 S.I. & & 3 S.I. & & 5 S.I & & $8 s$ & \\
\hline
\end{tabular}

36. $D_{7} \supset C_{2} \oplus B_{4} \supset C_{2} \oplus A_{1} \oplus A_{1} \supset A_{1} \oplus A_{1} \oplus A_{1} \oplus A_{1} \supset\left(A_{1}\right)_{13} \oplus A_{1} \oplus A_{1} \supset\left(A_{1}\right)_{23} \oplus A_{1}$.

\begin{tabular}{|c|c|c|c|c|c|c|c|c|c|c|c|}
\hline \multicolumn{2}{|l|}{$D_{7}$} & \multicolumn{2}{|c|}{$C_{2} \oplus B_{4}$} & \multicolumn{2}{|c|}{$C_{2} \oplus A_{1} \oplus A_{1}$} & \multicolumn{2}{|l|}{$\left(A_{1}\right)^{4}$} & \multicolumn{2}{|c|}{$\left(A_{1}\right)^{3}$} & \multicolumn{2}{|c|}{$\left(A_{1}\right)^{2}$} \\
\hline Peso & $d$ & Peso & $d$ & Peso & $d$ & Peso & $d$ & Peso & $d$ & Peso & $d$ \\
\hline \multirow[t]{8}{*}{$(0000010)$} & \multirow[t]{8}{*}{64} & \multirow[t]{8}{*}{$(10,0001)$} & \multirow[t]{8}{*}{64} & \multirow[t]{4}{*}{$(10,3,1)$} & \multirow[t]{4}{*}{32} & \multirow[t]{2}{*}{$(1,0,3,1)$} & \multirow[t]{2}{*}{16} & $(2,0,1)$ & 6 & $(1,2)$ & 6 \\
\hline & & & & & & & & $(4,0,1)$ & 10 & $(1,4)$ & 10 \\
\hline & & & & & & \multirow[t]{2}{*}{$(0,1,3,1)$} & \multirow[t]{2}{*}{16} & \multirow[t]{2}{*}{$(3,1,1)$} & \multirow[t]{2}{*}{16} & $(0,3)$ & 4 \\
\hline & & & & & & & & & & $(2,3)$ & 12 \\
\hline & & & & \multirow[t]{4}{*}{$(10,1,3)$} & \multirow[t]{4}{*}{32} & \multirow[t]{2}{*}{$(1,0,1,3)$} & \multirow[t]{2}{*}{16} & $(0,0,3)$ & 4 & $(3,0)$ & 4 \\
\hline & & & & & & & & $(2,0,3)$ & 12 & $(3,2)$ & 12 \\
\hline & & & & & & \multirow[t]{2}{*}{$(0,1,1,3)$} & \multirow[t]{2}{*}{16} & \multirow[t]{2}{*}{$(1,1,3)$} & \multirow[t]{2}{*}{16} & $(2,1)$ & 6 \\
\hline & & & & & & & & & & $(4,1)$ & 10 \\
\hline \multicolumn{2}{|l|}{1 S.I. } & \multicolumn{2}{|l|}{1 S.I. } & \multicolumn{2}{|l|}{2 S.I. } & \multicolumn{2}{|l|}{4 S.I. } & \multicolumn{2}{|c|}{6 S.I. } & \multicolumn{2}{|c|}{8 S.I. } \\
\hline
\end{tabular}


37. $D_{7} \supset A_{1} \oplus A_{1} \oplus D_{5} \supset A_{1} \oplus A_{1} \oplus A_{4} \supset A_{1} \oplus A_{1} \oplus A_{3} \supset A_{1} \oplus A_{1} \oplus A_{1} \oplus A_{1} \supset\left(A_{1}\right)_{13} \oplus A_{1} \oplus A_{1} \supset\left(A_{1}\right)_{23} \oplus A_{1}$.

\begin{tabular}{|c|c|c|c|c|c|c|c|c|c|c|c|c|c|}
\hline \multicolumn{2}{|l|}{$D_{7}$} & \multicolumn{2}{|c|}{$\left(A_{1}\right)^{2} \oplus D_{5}$} & \multicolumn{2}{|c|}{$\left(A_{1}\right)^{2} \oplus A_{4}$} & \multicolumn{2}{|c|}{$\left(A_{1}\right)^{2} \oplus A_{3}$} & \multicolumn{2}{|l|}{$\left(A_{1}\right)^{4}$} & \multicolumn{2}{|c|}{$\left(A_{1}\right)^{3}$} & \multicolumn{2}{|c|}{$\left(A_{1}\right)^{2}$} \\
\hline Peso & $d$ & Peso & $d$ & Peso & $d$ & Peso & $d$ & Peso & $d$ & Peso & $d$ & Peso & $d$ \\
\hline \multirow[t]{18}{*}{$(0000010)$} & \multirow[t]{18}{*}{64} & \multirow[t]{9}{*}{$(0,1,00010)$} & \multirow[t]{9}{*}{32} & \multirow[t]{3}{*}{$(0,1,1000)$} & \multirow[t]{3}{*}{10} & \multirow[t]{2}{*}{$(0,1,100)$} & \multirow[t]{2}{*}{8} & \multirow[t]{2}{*}{$(0,1,1,1)$} & \multirow[t]{2}{*}{8} & \multirow[t]{2}{*}{$(1,1,1)$} & \multirow[t]{2}{*}{8} & $(0,1)$ & 2 \\
\hline & & & & & & & & & & & & $(2,1)$ & 6 \\
\hline & & & & & & $(0,1,000)$ & 2 & $(0,1,0,0)$ & 2 & $(0,1,0)$ & 2 & $(1,0)$ & 2 \\
\hline & & & & \multirow[t]{5}{*}{$(0,1,0010)$} & \multirow[t]{5}{*}{20} & \multirow[t]{3}{*}{$(0,1,010)$} & \multirow[t]{3}{*}{12} & $(0,1,2,0)$ & 6 & $(2,1,0)$ & 6 & $(1,2)$ & 6 \\
\hline & & & & & & & & \multirow[t]{2}{*}{$(0,1,0,2)$} & \multirow[t]{2}{*}{6} & \multirow[t]{2}{*}{$(0,1,2)$} & \multirow[t]{2}{*}{6} & $(1,0)$ & 2 \\
\hline & & & & & & & & & & & & $(3,0)$ & 4 \\
\hline & & & & & & \multirow[t]{2}{*}{$(0,1,001)$} & \multirow[t]{2}{*}{8} & \multirow[t]{2}{*}{$(0,1,1,1)$} & \multirow[t]{2}{*}{8} & \multirow[t]{2}{*}{$(1,1,1)$} & 8 & $(0,1)$ & 2 \\
\hline & & & & & & & & & & & & $(2,1)$ & 6 \\
\hline & & & & $(0,1,0000)$ & 2 & $(0,1,000)$ & 2 & $(0,1,0,0)$ & 2 & $(0,1,0)$ & 2 & $(1,0)$ & 2 \\
\hline & & $(1,0,00001)$ & 32 & $(1,0,0001)$ & 10 & $(1,0,001)$ & 8 & $(1,0,1,1)$ & 8 & $(0,0,1)$ & 2 & $(1,0)$ & 2 \\
\hline & & & & & & & & & & $(2,0,1)$ & 6 & $(1,2)$ & 6 \\
\hline & & & & & & $(1,0,000)$ & 2 & $(1,0,0,0)$ & 2 & $(1,0,0)$ & 2 & $(0,1)$ & 2 \\
\hline & & & & $(1,0,0100)$ & 20 & $(1,0,010)$ & 12 & $(1,0,2,0)$ & 6 & $(1,0,0)$ & 2 & $(0,1)$ & 2 \\
\hline & & & & & & & & & & $(3,0,0)$ & 4 & $(0,3)$ & 4 \\
\hline & & & & & & & & $(1,0,0,2)$ & 6 & $(1,0,2)$ & 6 & $(2,1)$ & 6 \\
\hline & & & & & & $(1,0,100)$ & 8 & $(1,0,1,1)$ & 8 & $(0,0,1)$ & 2 & $(1,0)$ & 2 \\
\hline & & & & & & & & & & $(2,0,1)$ & 6 & $(1,2)$ & 6 \\
\hline & & & & $(1,0,0000)$ & 2 & $(1,0,000)$ & 2 & $(1,0,0,0)$ & 2 & $(1,0,0)$ & 2 & $(0,1)$ & 2 \\
\hline 1 S.I. & & 2 S.I. & & 6 S.I. & & 10 S.I & & 12 S.I. & & $15 \mathrm{~s}$ & & 18 & \\
\hline
\end{tabular}

38. $D_{7} \supset C_{2} \oplus B_{4} \supset C_{2} \oplus A_{1} \oplus A_{1} \oplus C_{2} \supset C_{2} \oplus A_{1} \oplus A_{1} \oplus A_{1} \supset A_{1} \oplus A_{1} \oplus A_{1} \oplus A_{1} \supset\left(A_{1}\right)_{12} \oplus A_{1} \oplus A_{1} \supset\left(A_{1}\right)_{23} \oplus A_{1}$.

\begin{tabular}{|c|c|c|c|c|c|c|c|c|c|c|c|c|c|}
\hline \multicolumn{2}{|l|}{$D_{7}$} & \multicolumn{2}{|c|}{$C_{2} \oplus B_{4}$} & \multicolumn{2}{|c|}{$C_{2} \oplus\left(A_{1}\right)^{2} \oplus C_{2}$} & \multicolumn{2}{|c|}{$C_{2} \oplus\left(A_{1}\right)^{3}$} & \multicolumn{2}{|l|}{$\left(A_{1}\right)^{4}$} & \multicolumn{2}{|c|}{$\left(A_{1}\right)^{3}$} & \multicolumn{2}{|c|}{$\left(A_{1}\right)^{2}$} \\
\hline Peso & $d$ & Peso & $d$ & Peso & $d$ & Peso & $d$ & Peso & $d$ & Peso & $d$ & Peso & $d$ \\
\hline \multirow[t]{4}{*}{$(0000010)$} & \multirow[t]{4}{*}{64} & \multirow[t]{4}{*}{$(10,0001)$} & \multirow[t]{4}{*}{64} & \multirow[t]{2}{*}{$(10,1,0,10)$} & \multirow[t]{2}{*}{32} & \multirow[t]{2}{*}{$(10,1,0,3)$} & \multirow[t]{2}{*}{32} & \multirow[t]{2}{*}{$(3,1,0,3)$} & \multirow[t]{2}{*}{32} & $(2,0,3)$ & 12 & $(3,2)$ & 12 \\
\hline & & & & & & & & & & $(4,0,3)$ & 20 & $(3,4)$ & 20 \\
\hline & & & & \multirow[t]{2}{*}{$(10,0,1,10)$} & \multirow[t]{2}{*}{32} & \multirow[t]{2}{*}{$(10,0,1,3)$} & \multirow[t]{2}{*}{32} & \multirow[t]{2}{*}{$(3,0,1,3)$} & \multirow[t]{2}{*}{32} & \multirow[t]{2}{*}{$(3,1,3)$} & \multirow[t]{2}{*}{32} & $(2,3)$ & 12 \\
\hline & & & & & & & & & & & & $(4,3)$ & 20 \\
\hline \multicolumn{2}{|l|}{1 S.I. } & \multicolumn{2}{|l|}{1 S.I. } & \multicolumn{2}{|l|}{2 S.I. } & \multicolumn{2}{|l|}{2 S.I. } & \multicolumn{2}{|l|}{2 S.I. } & \multicolumn{2}{|c|}{3 S.I. } & \multicolumn{2}{|c|}{4 S.I. } \\
\hline
\end{tabular}


39. $D_{7} \supset A_{1} \oplus A_{1} \oplus D_{5} \supset A_{1} \oplus A_{1} \oplus A_{4} \supset A_{1} \oplus A_{1} \oplus A_{1} \oplus A_{2} \supset A_{1} \oplus A_{1} \oplus A_{1} \oplus \tilde{A}_{1} \supset\left(A_{1}\right)_{13} \oplus A_{1} \oplus A_{1} \supset\left(A_{1}\right)_{23} \oplus A_{1}$.

\begin{tabular}{|c|c|c|c|c|c|c|c|c|c|c|c|c|c|}
\hline \multicolumn{2}{|l|}{$D_{7}$} & \multicolumn{2}{|c|}{$\left(A_{1}\right)^{2} \oplus D_{5}$} & \multicolumn{2}{|c|}{$\left(A_{1}\right)^{2} \oplus A_{4}$} & \multicolumn{2}{|c|}{$\left(A_{1}\right)^{3} \oplus A_{2}$} & \multicolumn{2}{|l|}{$\left(A_{1}\right)^{4}$} & \multicolumn{2}{|c|}{$\left(A_{1}\right)^{3}$} & \multicolumn{2}{|c|}{$\left(A_{1}\right)^{2}$} \\
\hline Peso & $d$ & Peso & $d$ & Peso & $d$ & Peso & $d$ & Peso & $d$ & Peso & $d$ & Peso & $d$ \\
\hline \multirow[t]{17}{*}{$(0000010)$} & \multirow[t]{17}{*}{64} & \multirow[t]{9}{*}{$(0,1,00010)$} & \multirow[t]{9}{*}{32} & \multirow[t]{3}{*}{$(0,1,1000)$} & \multirow[t]{3}{*}{10} & \multirow[t]{2}{*}{$(0,1,0,10)$} & \multirow[t]{2}{*}{6} & \multirow[t]{2}{*}{$(0,1,0,2)$} & \multirow[t]{2}{*}{6} & \multirow[t]{2}{*}{$(0,1,2)$} & \multirow[t]{2}{*}{6} & $(1,0)$ & 2 \\
\hline & & & & & & & & & & & & $(3,0)$ & 4 \\
\hline & & & & & & $(0,1,1,00)$ & 4 & $(0,1,1,0)$ & 4 & $(1,1,0)$ & 4 & $(1,1)$ & 4 \\
\hline & & & & \multirow[t]{5}{*}{$(0,1,0010)$} & \multirow[t]{5}{*}{20} & \multirow[t]{2}{*}{$(0,1,1,01)$} & \multirow[t]{2}{*}{12} & \multirow[t]{2}{*}{$(0,1,1,2)$} & \multirow[t]{2}{*}{12} & \multirow[t]{2}{*}{$(1,1,2)$} & \multirow[t]{2}{*}{12} & $(1,1)$ & 4 \\
\hline & & & & & & & & & & & & $(3,1)$ & 8 \\
\hline & & & & & & \multirow[t]{2}{*}{$(0,1,0,10)$} & \multirow[t]{2}{*}{6} & \multirow[t]{2}{*}{$(0,1,0,2)$} & \multirow[t]{2}{*}{6} & \multirow[t]{2}{*}{$(0,1,2)$} & 6 & $(1,0)$ & 2 \\
\hline & & & & & & & & & & & & $(3,0)$ & 4 \\
\hline & & & & & & $(0,1,0,00)$ & 2 & $(0,1,0,0)$ & 2 & $(0,1,0)$ & 2 & $(1,0)$ & 2 \\
\hline & & & & $(0,1,0000)$ & 2 & $(0,1,0,00)$ & 2 & $(0,1,0,0)$ & 2 & $(0,1,0)$ & 2 & $(1,0)$ & 2 \\
\hline & & $(1,0,00001)$ & 32 & $(1,0,0001)$ & 10 & $(1,0,0,01)$ & 6 & $(1,0,0,2)$ & 6 & $(1,0,2)$ & 6 & $(2,1)$ & 6 \\
\hline & & & & & & $(1,0,1,00)$ & 4 & $(1,0,1,0)$ & 4 & $(0,0,0)$ & 1 & $(0,0)$ & 1 \\
\hline & & & & & & & & & & $(2,0,0)$ & 3 & $(0,2)$ & 3 \\
\hline & & & & $(1,0,0100)$ & 20 & $(1,0,1,10)$ & 12 & $(1,0,1,2)$ & 12 & $(0,0,2)$ & 3 & $(2,0)$ & 3 \\
\hline & & & & & & & & & & $(2,0,2)$ & 9 & $(2,2)$ & 9 \\
\hline & & & & & & $(1,0,0,01)$ & 6 & $(1,0,0,2)$ & 6 & $(1,0,2)$ & 6 & $(2,1)$ & 6 \\
\hline & & & & & & $(1,0,0,00)$ & 2 & $(1,0,0,0)$ & 2 & $(1,0,0)$ & 2 & $(0,1)$ & 2 \\
\hline & & & & $(1,0,0000)$ & 2 & $(1,0,0,00)$ & 2 & $(1,0,0,0)$ & 2 & $(1,0,0)$ & 2 & $(0,1)$ & 2 \\
\hline 1 S.I. & & 2 S.I. & & 6 S.I. & & 12 S.I. & & 12 S.I. & & $14 \mathrm{~s}$ & & $17 \mathrm{~s}$ & \\
\hline
\end{tabular}





\section{Bibliografia}

[1] J.F. Adams: Lectures on Lie Groups, W.A. Benjamin, Amsterdam (1969).

[2] A. Barut \& R. Rạczka: Theory of Group Representations and Applications, 2nd edition, World Scientific Publishing, Singapore (1986).

[3] L. Braggion: Procura por Simetrias de Lie na evolução do Código Genético, Dissertação de Mestrado, ICMSC-USP-São Carlos (1998).

[4] A. Borel \& J. de Siebenthal: Les sous-groupes fermés de rang maximum des groupes de Lie clos, Comment. Math. Helv. 23(1949), 200-221.

[5] M. Bordemann, M. Forger \& H. Römer: Homogeneous Kähler manifolds: Paving the way towards new supersymmetric Sigma models, Commun. Math. Phys. 102(1986), 605-647.

[6] N. Bourbaki: Lie Groups and Lie Algebras, Chapters 1-8, Hermann, Paris (1975).

[7] T. Bröcker \& T. tomDieck: Representations of Compact Lie Groups, 2nd printing, Springer-Verlag, New York (1995).

[8] C. Chevalley: Theory of Lie Groups, Princeton University Press, Princeton (1946).

[9] J. Dixmier: Enveloping Algebras, Graduate Studies in Mathematics, Volume 11, American Mathematical Society, Providence, R.I. (1996).

[10] E.B. Dynkin: Semisimple subalgebras of semisimple Lie algebras, Mat. Sb. 30(1952) 349-462; English transl.: AMS Translations(2) 6(1957), 111-244.

[11] E.B. Dynkin: Maximal subgroups of classical groups, Trudy Moskov. Mat. Obshch. 1(1952) 39-166; English transl.: AMS Translations(2) 6(1957), 245-378. 
[12] M. Forger: Symmetry breaking in the genetic code, $41^{\circ}$ Seminário Brasileiro de Análise, Campinas (1995).

[13] M. Forger: Invariant polynomials and Molien functions, J. Math. Phys. 39(1998), n 2, 1107-1141.

[14] M. Forger, J.E.M. Hornos \& Y.M.M. Hornos: Global aspects in the algebraic approach to the genetic code, Phys. Rev. E, 56(1997), 7078-7082.

[15] W. Fulton \& J. Harris: Representation Theory: A First Course, Springer-Verlag, New York (1991).

[16] M. Golubitsky: Primitive actions and maximal subgroups of Lie groups, J. Differential Geometry 7(1972), 175-191.

[17] M. Golubitsky \& B. Rothschild: Primitive subalgebras of exceptional Lie algebras, Pacific Journal of Mathematics 39(1971), n 2, 371-393.

[18] M. Goto \& F.D. Grosshans: Semisimple Lie Algebras, Lecture Notes in Pure and Applaied Mathematics, Volume 38, Marcel Dekker, New York (1978).

[19] S. Helgason: Differential Geometry, Lie Groups and Symmetric Spaces, Academic Press, New York (1978).

[20] J.E. Humphreys: Introduction to Lie Algebras and Representation Theory, 3rd printing, Springer-Verlag, New York (1994).

[21] G. Hochschild: The Structure of Lie Groups, Holden-Day, San Francisco (1965).

[22] J.E.M. Hornos \& Y.M.M. Hornos: Algebraic Model for the Evolution of the Genetic Code, Phys. Rev. Lett. 71(1993), 4401-4404.

[23] J.E.M. Hornos, Y.M.M. Hornos \& M. Forger: Symmetry and Symmetry Breaking: An Algebraic Approach to the Genetic Code, Int. J. Mod. Phys. B 13(1999) 2795-2885.

[24] N. Jacobson: Lie Algebras, Dover Publications, New York (1997).

[25] F.I. Karpelevich, A.L. Onishchik \& E.B. Vinberg : On the Work of E.B. Dynkin in the Theory of Lie Groups, AMS Translations(2) 169(1995), 1-13.

[26] A.W. Knapp: Representation Theory of Semisimple Lie Groups, Princeton University Press, New Jersey (1986).

[27] A.W. Knapp: Lie Groups, Lie Algebras and Cohomology, Princeton University Press, New Jersey (1988). 
[28] A.W. Knapp: Lie Groups Beyond an Introduction, Birkhäuser, Boston (1996).

[29] A.A. Kirilov: Elements of the Theory of Representations, Springer-Verlag, New York (1976).

[30] B.P. Komrakov: Reductive subalgebras of real semisimple Lie algebras, Dokl. Akad. Nauk SSSR, 308(1989), 521-525. English transl.: Sov. Math. Dokl. 40(1990), 329-333.

[31] B.P. Komrakov: Maximal subalgebras of real Lie algebras and a problem of Sophus Lie, Dokl. Akad. Nauk SSSR, 311(1990), 528-532. English transl.: Sov. Math. Dokl. 41(1990), 269-273.

[32] H. Kopka \& P.W. Daly: A Guide to $\mathrm{HT}_{\mathrm{E}} \mathrm{X} 2_{\varepsilon}$, 2nd edition, Addison-Wesley, Reading, Massachusetts (1996).

[33] J. Maddox: The genetic code by numbers, Nature 367(1994), 111.

[34] A.I. Malcev: On semisimple subgroups of Lie groups, Izv. Akad. Nauk SSSR 8(1944), 143-174; English transl.: AMS Translations(1) 9(1962), 172-213.

[35] W.G. McKay \& J. Patera: Tables of Dimensions, Indices and Branching Rules of Simple Lie Algebras, Lecture Notes in Pure and Applaied Mathematics, Volume 69, Marcel Dekker, New York (1981).

[36] W.G. McKay, J. Patera \& D. Sankoff: The Computation of Branching Rules for Repesentations of Semisimple Lie Algebras, Computers in Nonassociative Rings and Algebras, R.E. Beck \& B. Kolman (Eds.), Academic Press, New York (1977).

[37] G.D. Mostow: The Extensibility of Local Lie groups of Transformations and Groups on Surfaces, Annals of Mathematics 52(1950), n 3, 606-636.

[38] G.D. Mostow: On maximal subgroups of real Lie groups, Annals of Mathematics 74(1961), n $3,503-517$.

[39] M.A. Naimark \& A.I. Stern: Theory of Group Representation, Springer-Verlag, New York (1982).

[40] A. Navon \& J. Patera: Embedding of a simple Lie group into a simple Lie group and branching rules, J. Math. Phys. 8(1967), no 3, 489-493.

[41] A.L. Onishchik (Ed.): Lie Groups and Lie Algebras I: Foundations of Lie Theory. Lie Groups of Trandformations., Encyclopaedia of Mathematical Sciences Volume 20, Springer-Verlag, New York (1993). 
[42] A.L. Onishchik \& E.B. Vinberg (Eds.): Lie Groups and Lie Algebras III: Structure of Lie Groups and Lie Algebras, Encyclopaedia of Mathematical Sciences Volume 41, Springer-Verlag, New York (1994).

[43] A.L. Onishchik \& E.B. Vinberg: Lie Groups and Algebraic Groups, Springer-Verlag, New York (1990).

[44] A.A. Sagle \& R.E. Walde: Introduction to Lie Groups and Lie Algebras, Academic Press, New York (1973).

[45] H. Samelson: Notes on Lie Algebras, Springer-Verlag, New York (1990).

[46] D.H. Sattinger \& O.L. Weaver: Lie Groups and Algebras with Applications to Physics, Geometry and Mechanics, Springer-Verlag, New York (1986).

[47] J.-P. Serre: Complex Semisimple Lie Algebras, Springer-Verlag, New York (1987).

[48] J.-P. Serre: Lie Algebra and Lie Groups, 2nd edition, Lecture Notes in Mathematics 1500, Springer-Verlag, New York (1992).

[49] S. Sternberg: Group Theory and Physics, Cambridge University Press, London (1994).

[50] I. Stewart: Broken symmetry in the genetic code ?, New Scientist (5 MArch 1994), 16.

[51] Tao Hui-Min: The maximal nonsemisimple subalgebras of a noncompact real semisimple Lie algebra, Acta Math. Sin. 16(1966), no2, 253-268. English transl.: Chin. Math. 8(1966), 265-282.

[52] M.S. Taufik: On maximal subalgebras in classical real Lie algebras, Vopr. Teor. Grupp Gromol. Algebry, 2(1979), 148-168. English transl.: Sel. Math. Sov. 6(1987), 163-176.

[53] J. Tits: Sous-algèbres des algèbres de Lie semi-simples, Séminaire Bourbaki, Mai 1995.

[54] V.S. Varadarajan: Lie Groups, Lie Algebras and Their Representations, SpringerVerlag, New York (1984).

[55] N.R. Wallach: Real Redutctive Groups I, Academic Press, New York (1988).

[56] F.W. Warner: Foundations of Differentiable Manifolds and Lie Groups, Springer-Verlag, New York (1983).

[57] G. Warner: Harmonic Analysis on Semi-Simple Lie Groups I \& II, Springer-Verlag, New York (1972). 
[58] H. Weyl: Classical Groups, Their Invariants and Representaitions, 2nd edition, Princeton University Press, Princeton (1946).

[59] H. Weyl: Simetria, EdUSP, São Paulo (1997).

[60] E.P. Wigner: The Unreasonable Effectiveness of Mathematicas in Natural Sciences, Comm. on Pure and Applied Math., 13(1960), 01-14.

[61] D.P. Želobenko: Compact Lie Groups and Their Representations, Translations of Mathematical Monographs, Volume 40, American Mathematical Society, Providence, R.I. (1973). 